\title{
Tracking with all-analog adaptive antenna arrays
}

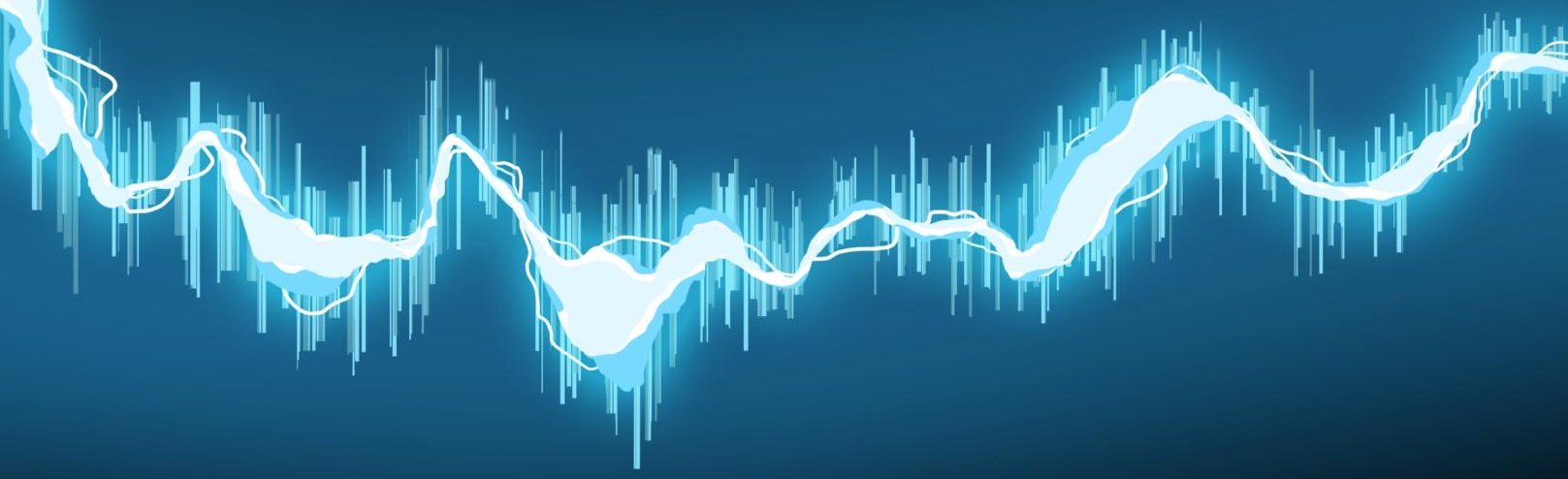

Tom M. Bruintjes 
Members of the graduation committee:

Prof. dr. ir. P. G. M. Apers

Prof. dr. ir. G. J. M. Smit

Dr. ir. A. B. J. Kokkeler

Dr. ir. G. Karagiannis

Prof. dr. ir. F. E. van Vliet

Dr. ir. M. J. Bentum

Prof. dr. ir. A. M. J. Koonen

Prof. dr. ir. S. M. Heemstra de Groot
University of Twente (chairman and secretary)

University of Twente (promotor)

University of Twente (assistant promotor)

Huawei Technologies Co. Ltd.

University of Twente / TNO

University of Twente / ASTRON

Eindhoven University of Technology

Eindhoven University of Technology

\section{UNIVERSITY OF TWENTE.}

Faculty of Electrical Engineering, Mathematics and Computer Science, Computer Architecture for Embedded Systems (CAES) group

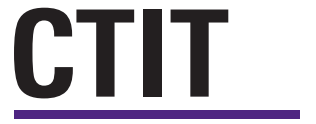

NWO
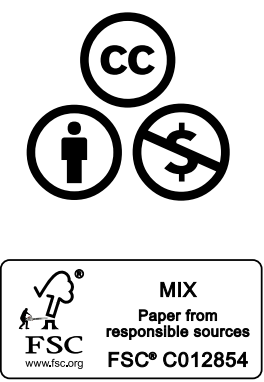

CTIT Ph.D. Thesis Series No. 15-380

Centre for Telematics and Information Technology

PO Box 217, 7500 AE Enschede, The Netherlands

The research in this thesis has been conducted within the SOWICI project (project number 647.000.005), which was financed by the Netherlands Organization for Scientific Research.

Copyright $\odot 2015$ Tom M. Bruintjes, Enschede, The Netherlands. This work is licensed under the Creative Commons AttributionNonCommercial 4.o International License. To view a copy of this license, visit http://creativecommons.org/licenses/ by-nc/4.0/deed.en_US.

Typeset with LATE, TikZ and Gnuplot.

Printed by Gildeprint Drukkerijen, The Netherlands.

ISBN 978-90-365-4006-3

ISSN 1381-3617; CTIT Ph.D. Thesis Series No. 15-38o

DOI $10.3990 / 1.9789036540063$ 


\section{TRACKING WITH ALL-ANALOG}

ADAPTIVE ANTENNA ARRAYS

\section{ProefsChRIFT}

ter verkrijging van

de graad van doctor aan de Universiteit Twente,

op gezag van de rector magnificus, prof. dr. H. Brinksma,

volgens besluit van het College voor Promoties

in het openbaar te verdedigen

op vrijdag 18 december 2015 om 14.45 uur

door

Thomas Maarten Bruintjes

geboren op 4 juni 1985

te Wijk bij Duurstede 
Dit proefschrift is goedgekeurd door:

Prof. dr. ir. G. J. M. Smit (promotor)

Dr. ir. A. B. J. Kokkeler (assistent-promotor)

Copyright (c) 2015 Tom M. Bruintjes

ISBN 978-90-365-4006-3 


\section{Abstract}

Wireless communications exist on the most remote corners of the Earth. Even high up in the mountains one checks the weather forecast by smartphone these days. The expanding capacity requirements of wireless networks have led to spectrum scarcity. Most people will, at one point, have experienced a poor Wi-Fi connection because of too many active $2.4 \mathrm{GHz}$ devices in the vicinity. Millimeter waves are a promising candidate to solve this problem. Especially at $60 \mathrm{GHz}$, where there is roughly (depending on the continental regulations) $4-9 \mathrm{GHz}$ of unlicensed spectrum available.

Because of the high-speed (multiple gigabits per second) communication potential of millimeter waves, they are ideally suited to be integrated with fiber optical networks. In particular, this thesis considers an in-door scenario in which each room of a building is equipped with a mm-wave radio access point connected to a fiber backbone. As $60 \mathrm{GHz}$ does not penetrate walls, frequencies can be reused in neighboring rooms. However, $60 \mathrm{GHz}$ carriers also require the use of antennas that feature high gain and directionality. For indoor use, a steerable dish antenna is not desirable. Instead an array of simple (patch) antennas is employed. By combining the signals collected through multiple antennas, and giving each of these channels an appropriate delay (and amplification), constructive and destructive interference give rise to high gain and directionality as well.

The mobility of wireless devices demands that this beamforming is performed adaptively. In other words, the beam that is formed by constructive interference needs to be locked onto the moving targets to maintain a proper connection. With antenna arrays this is accomplished efficiently by means of imposing the right delays, which is called electronic- or optical steering. Furthermore, from a cost- and energy efficiency point of view, it is desirable to implement the beamforming network(s) entirely in the analog domain and to control adaptivity in a centralized manner. A consequence of this combination is that the combined beamformer output will be the only source of information for signal processing; a considerable limitation that requires a novel algorithm to track the position of the mobile devices. This algorithm is the primary objective of this thesis.

To estimate a signal's angle of incidence (i.e., the angular position of the source), and to optimize the beamformer's output to this angle, the majority of adaptive array algorithms operates on the basis of cross-correlating individually sampled antenna signals. With (remotely controlled) all-analog beamforming this is not an option. Current solutions require that the environment is scanned with a single 
beam, which is inefficient in a communication scenario. Partially, this inefficiency can be explained by the symmetric piriform shaped beam of a conventional beamformer. Tracking based on the use of asymmetrically shaped beampatterns is proposed for improvement. The secondary objective of this thesis is to synthesize these unconventionally shaped beampatterns. Synthesis refers to employing mathematical techniques to find delay and amplification values, i.e. the array excitations, that yield the specified beampattern shape.

Both objectives are first investigated in the context of (equispaced) linear antenna arrays. A classical analytic approach is adopted for synthesis because it produces smooth shapes. Smoothness is an important property for the envisaged tracking algorithm. The reason hereof is that the latter exploits a known (unique) relation between a given angle and the power of the beamformer output, to estimate the angle of incidence. This unique relation is achieved with a ramp shaped beampattern. However, because the shape of the beampattern is not the only factor that influences received power (think of e.g., path loss), a second shape is introduced. This shape exhibits a power response that is independent of the angle (i.e., a flat shape). A tracking mechanism is developed in which the shapes are alternated, and the correct steering angle is found in the power difference between the two beampatterns. The algorithm is shown to work by means of Monte Carlo simulations.

To assess whether shaped-pattern tracking is also useful in a practical application, various realization aspects are covered. Firstly, modifications to the synthesis algorithm are presented to take into account electromagnetic phenomena that alter the shape of the beampattern. Special care is given to the non-isotropic gain of the individual antenna elements. Secondly, the excitation values needed for the shaped patterns are optimized from a realization perspective. This pertains mostly to reduction of the required channel amplification range. When these steps are performed correctly, realization of the proposed tracking mechanism is feasible.

Finally, as linear arrays only provide directionality in one dimension, the entire shaped-pattern tracking approach is extended to planar antenna arrays. Different array geometries are considered from which hexagonally shaped grid structures appear to be the most suitable. This has to do with resilience against deformation of the beam shape under different steering angles. Synthesizing shaped beampatterns for such an array structure is, however, far from trivial. A procedure based on the principle of collapsed distributions is employed and tailored to support asymmetry in the specified pattern shape. The result is a smooth asymmetric pattern generated by an array with high rotational symmetry. Because of the latter, the shape of the pattern suffers less from extreme steering angles. In addition, the collapsed distributions method is a direct extension of linear array synthesis. This means that it benefits from most of the aforementioned algorithmic improvements and properties.

In conclusion, based on simulations and realization considerations, this thesis shows that using shaped beampatterns can be an attractive means of tracking when the beamformer is entirely analog. 


\section{SAMENVATTING}

Draadloze communicatie komt voor in de verste uithoeken op Aarde. Zelfs hoog in de bergen wordt tegenwoordig per smartphone het weerbericht geraadpleegd. De toenemende eisen voor draadloos verkeer hebben echter gezorgd voor een frequentie schaarste. Veel lezers zullen wel eens te maken hebben gehad met een slechte Wi-Fi verbinding omdat er teveel actieve $2.4 \mathrm{GHz}$ apparatuur in de buurt is. Radiogolven in het millimeter bereik kunnen hier uitkomst bieden. In het bijzonder rondom $60 \mathrm{GHz}$, waar een band van ongeveer (afhankelijk van de regelgeving per continent) 4-9 GHz ligt die vrij te gebruiken is.

Omdat het gebruik van mm-golven uiterst breedbandige communicatie toestaat (gigabits per seconde) zijn deze bijzonder geschikt om geïntegreerd te worden met het glasvezelnetwerk. Dit proefschrift veronderstelt een scenario waarin elke kamer binnen een gebouw is uitgerust met een mm-golf access point-verbonden met het glasvezelnetwerk. Omdat $60 \mathrm{GHz}$ golven niet door muren heen dringen kunnen frequenties in naburige kamers hergebruikt worden. Echter, de antennes moeten dan beschikken over een hoge opbrengst en richtingsgevoeligheid. Voor binnengebruik is een instelbare schotelantenne niet wenselijk. In plaats daarvan wordt een array van simpele (patch) antennes gebruikt. Door signalen-ontvangen door meerdere antennes-te combineren en de corresponderende kanalen te voorzien van de juiste vertraging en versterking, worden door constructieve en destructieve interferentie de gewenste opbrengst en richtingsgevoeligheid ook verkregen.

De mobiliteit van draadloze apparatuur vereist adaptieve bundelvorming. Dat wil zeggen, de straalverbinding die wordt verkregen d.m.v. constructieve interferentie moet bewegende apparatuur kunnen volgen om een goede verbinding te kunnen waarborgen. Met antenne arrays kan dit efficiënt gerealiseerd worden door de juiste tijdsvertragingen aan te brengen op de verschillende kanalen, wat ook wel elektronisch bijsturen wordt genoemd. Vanuit een kosten- en energie-efficiëntie standpunt is het wenselijk om de bundelvorming volledig in het analoge domein te implementeren, en de adaptiviteit centraal te regelen. Die combinatie betekent dat enkel de gecombineerde uitgang van de bundelvormer een bron voor signaalverwerking is; een beperking welke de ontwikkeling van een nieuw volgalgoritme vereist. Dat algoritme is het primaire doel van dit proefschrift.

Om de invalshoek van een signaal te bepalen, en de bundelvormer daarop af te stemmen, bemonsteren veel algoritmes de idividuele antennes voor kruiscorrelatie. Bij centraal (op afstand) geregelde analoge bundelvorming is dat niet mogelijk. Huidige oplossingen vereisen dat de omgeving afgezocht wordt, wat inefficiënt is 
voor communicatiedoeleinden. Deels kan die inefficiëntie verklaard worden door de conventioneel symmetrisch gevormde bundel. Ter verbetering wordt daarom het gebruik van asymmetrisch gevormde bundels voorgesteld. Het secundaire doel van dit proefschrift is het synthetiseren van dergelijke bundels. Synthese impliceert het vinden van de juiste vertragingen en versterkingen, ofwel de array excitaties, die leiden tot de gewenste bundelvorm.

Beide doelen zullen eerst bestudeerd worden voor (equidistante) lineaire arrays. Een analytische methode zal toegepast worden voor synthese. De reden daarvoor is dat die methode in staat is de gewenste vorm met geringe rimpeling weet te benaderen. Deze eigenschap is belangrijk omdat het volgalgoritme is gefundeerd op een bekende en unieke relatie tussen gegeven hoek en het vermogen aan de uitgang van de bundelvormer. De unieke relatie wordt verkregen met een 'schuine' bundel. Echter, omdat de vorm van de bundel niet het enige is waardoor het vermogen wordt beïnvloed (denk bijvoorbeeld aan transmissieverlies), is het gebruik van een tweede vorm nodig. Deze bundel heeft (door zijn platte vorm) de eigenschap een vermogen op te leveren dat juist onafhankelijk is van de hoek. Het volgalgoritme werkt op basis van het afwisselen van de twee bovenbeschreven vormen, waarbij de correcte invalshoek bepaald wordt door het vermogensverschil tussen beide. De werking van dit principe wordt aangetoond door middel van Monte Carlo simulaties.

Om de realiseerbaarheid van het principe te beoordelen worden een aantal verschillende implementatieaspecten in overweging genomen. Ten eerste wordt het synthesealgoritme aangepast op het in acht nemen van elektromagnetische fenomenen die van invloed zijn op de bundelvorm. Speciale aandacht wordt daarbij geschonken aan de anisotropische opbrengst van de individuele antenne-elementen. Ten tweede worden de excitaties geoptimaliseerd voor implementatiedoeleinden. Dit heeft voornamelijk betrekking op het reduceren van de benodigde versterking. Wanneer beide stappen correct worden uitgevoerd zal het volgalgoritme op basis van (asymmetrisch) gevormde bundels geschikt zijn voor praktische toepassing.

Tot slot wordt de uitbreiding naar tweedimensionale arrays besproken, om meer richtingsgevoeligheid te creëren. Verschillende array structuren zijn overwogen waaruit blijkt dat een hexagonaal rooster het meest geschikt is. Dit heeft te maken met het behouden van de bundelvorm onder grote stuurhoeken. Het synthetiseren van bundels is echter niet triviaal voor deze structuur. Een procedure op basis van het collapsed distributions principe wordt daarvoor ontwikkeld. Het resultaat is een asymmetrische bundel gegenereerd door een array met een hoge mate van rotationele symmetrie. Die laatste eigenschap komt de vorm ten goede bij extreme stuurhoeken. Daarnaast is de procedure een directe uitbreiding op de toegepaste lineaire synthese, waardoor de eerder besproken optimalisaties en eigenschappen nog van toepassing zijn.

Concluderend, met dit proefschrift wordt aangetoond, door middel van simulaties en realisatieoverwegingen, dat bij analoge bundelvorming het gebruik maken van (asymmetrisch) gevormde bundels een aantrekkelijke oplossing kan zijn voor het volgen van mobiele gebruikers. 


\section{DANKWOORD}

Ik schrijf dit dankwoord als laatste onderdeel van mijn proefschrift en, net als bij het bedwingen van de laatste meters op een willekeurige topgraat in de Alpen, kosten deze twee pagina's nog venijnig veel inspanning. Enfin, de hele 'beklimming' heeft ongeveer vier jaar geduurd én een maand sponsoring van het UWV gekost. En hoewel ik het UWV dankbaar mag zijn, is dit dankwoord meer bedoeld voor de lieden die daadwerkelijk wat hebben bijgedragen aan het resultaat dat u voor zich ziet.

In de eerste plaats wil ik Gerard, André en Georgios bedanken. Gerard heeft mij de mogelijk gegeven te promoveren bij CAES—je kunt er over discussiëren maar mijns inziens is dat toch de mooiste vakgroep die er is-en daar ben ik hem zeer dankbaar voor. Ik heb mogen werken aan hobbyprojectjes en had tegelijkertijd (als uitzondering op de regel) de luxe van een vaste plek in Gerard zijn bomvolle agenda. Mijn tijd bij CAES heb ik daardoor als bijzonder plezierig ervaren. Van de wijze raad van André heb ik veelvuldig gebruik gemaakt om te zorgen dat hetgeen er na deze twee pagina's komt enige wetenschappelijke waarde heeft. Het proefschrift is er heel wat dikker van geworden, maar ik ben ervan overtuigd dat het daardoor een stuk beter leesbaar zal zijn. Georgios heeft met name in de eerste twee jaar van mijn promotietraject geholpen. Dit heeft ervoor gezorgd dat ik mijzelf niet direct heb vastgebeten in mijn eigen ideeën, maar ook eens wat verder heb gekeken. De overige commissieleden wil ik bedanken voor hun inbreng na het manuscript te hebben gelezen. Zizheng and Kishor, I also thank both of you for your pleasant cooperation in the SOWICI project.

Voor hun indirecte bijdrage aan een succesvolle afronding wil ik alle mensen die CAES maken wat het is bedanken voor de vier à vijf mooie jaren die ik er heb rondgelopen. Het gaat te ver om iedereen individueel te bedanken, maar van een aantal collega's/vrienden ga ik toch de naam noemen. Karel, jij was zo ongeveer de doorslaggevende factor voor mij om bij de vakgroep te blijven hangen als AiO. Het gepruts met Sabrewing heb ik altijd erg leuk gevonden, bedankt voor je begeleiding tijdens die periode. Christiaan, onze onderzoeksonderwerpen lagen mijlenver uit elkaar, maar onze interesses niet. De gesprekken tijdens koffiepauzes en lunchwandelingen, zij het over de fijnere details van plasma versus LCD of over de betere tactieken in Operation Locker, zijn altijd een bijzonder welkome afwisseling geweest. Jordy, van begin tot eind mijn trouwe kamergenoot. Onze dagelijkse discussies over de echt belangrijke vraagstukken (Hazet $\gg$ Snap-on, en hoe importeer ik een kettingzaag van $15 \mathrm{~kg}$ zo voordelig mogelijk uit de VS) ga ik zeker missen. Ik begin me inmiddels al af te vragen hoe het er met "de machine" voor staat. Gelukkig 
was Rinse er als mede-bergliefhebber. Ondertussen zit je al gevaarlijk dicht tegen mijn hoogterecord aan, ik hoop dat we die wedloop nog lang door kunnen zetten. Marlous, Nicole en Thelma wil ik graag bedanken voor het uit handen nemen van de papiermolen (de UT blinkt er in uit), maar ook voor het organiseren van meesterlijke vakgroepsuitjes.

Buiten werktijd heb ik zowel als student als AiO aardig wat tijd 'verspild' in De Rode Bar. Freark, Djurre, Bart, van AK-47 schieten in het Oostblok tot aan de vaste film- en game-sessies, ik heb het allemaal prachtig gevonden. Bedankt voor de broodnodige afleiding. En het mag gezegd worden: Freark, bijzonder tof dat je als eenmans-NL-divisie van Qualcomm terug komt uit de VS voor mijn verdediging.

Tot slot wil ik mijn familie bedanken. Pa, ma, bij dezen bedank ik jullie eens officieel voor alle ondersteuning en voor een beregezellig thuis in Epe. Daarvoor trotseer ik graag de ergernissen van het OV. Harold, "I'm seeing predictable phase arrays". Te mooi om te laten liggen gezien de inhoud van dit boekje. Bedankt voor het af en toe meedenken en het plaats willen nemen in mijn knokploeg tijdens de verdediging. Met genoegen zal ik hetzelfde doen bij jouw promotie over een jaar (of twee). Maudy, bedankt voor het helpen met de kaft, de gebroeders Schmid zouden er trots op zijn geweest. Mijn dank ook voor alle culinaire ondersteuning de afgelopen jaren, en je hebt je verbazingwekkend goed gedragen als huisgenoot.

Tom

Enschede, november 2015 


\section{Contents}

1 INTRODUCTION 1

$1.160 \mathrm{GHz}$ wireless communication ................... 2

1.1 .1 Antenna arrays ...................... 2

1.1.2 Optical-wireless integration . . . . . . . . . . . 3

$1.1 .3 \quad$ SOWICI ............................. 4

1.2 Problem statement . . . . . . . . . . . . . . . . 5

1.3 Approach ........................... 6

1.4 Outline ........................... 7

1.5 Conventions ........................... 7

2 ANTENNA ARRAY PRINCIPLES AND RELATED WORK 9

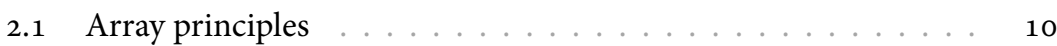

$2.1 .1 \quad$ Structure . . . . . . . . . . . . . . . 10

2.1.2 Response......................... 11

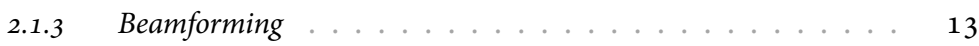

2.1.4 Beampattern...................... 14

$2.1 .5 \quad$ Beam steering . . . . . . . . . . . . . . . 18

2.2 Optical/analog beamforming . . . . . . . . . . . . 19

2.3 Adaptive arrays ... . . . . . . . . . . . . . 20. 20

$2.3 .1 \quad$ Partially adaptive ..................... 21

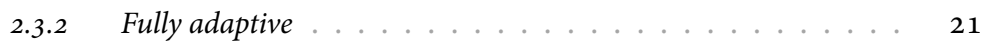

2.3.3 Compatibility and approach ................ 24

2.4 Conclusions . . . . . . . . . . . . . . . . . . . . . 26

3 LINEAR ARRAYS: ANALYSIS AND SYNTHESIS

3.1 Analysis . . . . . . . . . . . . . . . . . . . . . 28

3.1.1 The Schelkunoff transformation ........... 30

3.2 Synthesis based on polynomials ... . . . . . . . 34

3.2.1 Example: angular root displacement synthesis . . . . . . . 36

$3.2 .2 \quad$ Null-free shaped regions . . . . . . . . . . . . 37 
3.2.3 Example: radial root displacement synthesis . . . . . . . 39

3.3 Orchard-Elliott shaped-pattern synthesis . . . . . . . . 40

3.3.1 Example: balanced ripple synthesis . . . . . . . . 45

3.4 Beampatterns for tracking purposes . . . . . . . . . . 46

3.4.1 Asymmetrically shaped beam ................. 46

3.4.2 Uniform sidelobe suppression . . . . . . . . . . 48

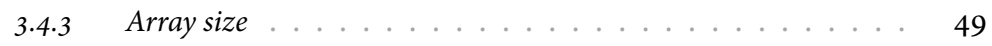

3.5 Related work ... . . . . . . . . . . . 50 50

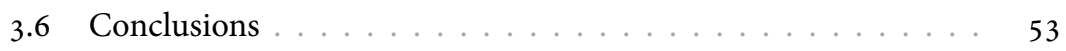

4 TRACKING BY MEANS OF SHAPED BEAMPATTERNS 55

4.1 Relative power .................. 56

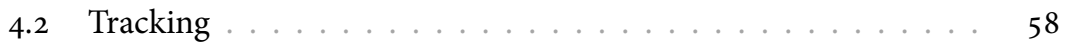

4.2.1 Normalization . . . . . . . . . . . . . . . 59

4.2.2 Trigger and direction ................... 59

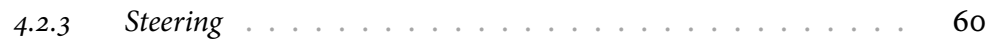

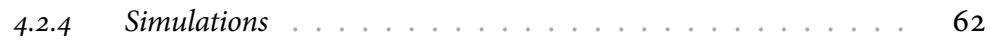

4.2 .5 Ripple tolerance . . . . . . . . . . . . 65

4.3 Direction-of-arrival estimation . . . . . . . . . . . . 66

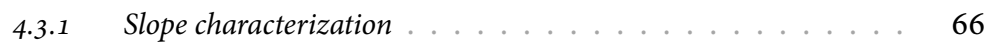

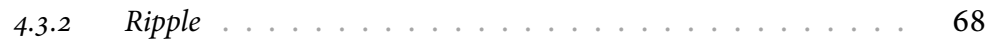

$4.3 .3 \quad$ Estimation accuracy ................ 69

4.4 Discussion and future work ................. 71

4.5 Conclusions ........................... 71

5 REALIZATION CONSIDERATIONS $\quad 73$

5.1 Element factor compensation . . . . . . . . . . . . 74

5.1.1 Analysis ........................ 74

5.1.2 Incorporating the element factor ........... 76

5.1.3 Application to a ramp shaped pattern .......... 80

5.1.4 Computational stability . . . . . . . . . . . . 81

5.1 .5 Discussion . . . . . . . . . . . . . 82

5.2 Mutual coupling considerations ............. 83

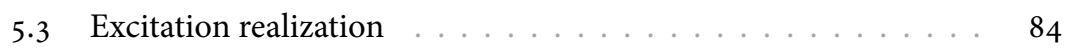

$5.3 .1 \quad$ Amplitude .................... 84

$5.3 .2 \quad$ Phase ....................... 87

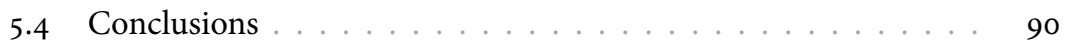


6.1 Analysis . . . . . . . . . . . . . . . . . . . 92

6.2 Synthesis . . . . . . . . . . . . . . . . . . . . . 95

$6.2 .1 \quad$ Synthesis via separable weightings . . . . . . . . . . . 95

$6.2 .2 \quad$ Hexagonal arrays . . . . . . . . . . . . . . . 98

$6.2 .3 \quad$ Related Work . . . . . . . . . . . . . 99

6.3 Synthesis via collapsed distributions . . . . . . . . . . 101

$6.3 .1 \quad$ Collapsing planar arrays . . . . . . . . . . . . 102

6.3.2 Collapsed distributions and linear synthesis . . . . . . . . 104

6.3.3 Planar synthesis by spreading collapsed distributions . . . . 106

6.3.4 Example: pencil beam with $\phi$-invariant sidelobes . . . . . . 107

6.4 Tracking patterns for hexagonal arrays . . . . . . . . . 111

6.4.1 Equispaced linear distributions . . . . . . . . . . . . 111

$6.4 .2 \quad$ Linear synthesis . . . . . . . . . . . . . . . . 111

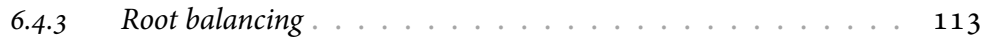

6.4 .4 Common excitation normalization . . . . . . . . . 115

6.4 .5 Expanding large polynomials . . . . . . . . . . 117

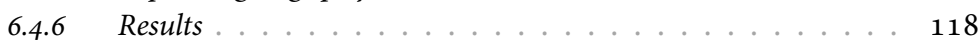

6.5 Steering and shape preservation ... . . . . . . 120

$6.5 .1 \quad$ Steering . . . . . . . . . . . . . . 120

$6.5 .2 \quad$ Tracking compatibility . . . . . . . . . . 121

6.6 Future work . . . . . . . . . . . . . . . . . 122

6.7 Conclusions . . . . . . . . . . . . . . . . . . . 124

7 CONCLUSIONS AND RECOMMENDATIONS $\quad \mathbf{1 2 7}$

7.1 Contributions . . . . . . . . . . . . . . . . 128

7.2 Recommendations ..................... . . 129

$\begin{array}{lll}\text { A Numerical DATA } & 131\end{array}$

$\begin{array}{lr}\text { ACRONYMS } & 135\end{array}$

$\begin{array}{lr}\text { NOMENCLATURE } & 137\end{array}$

$\begin{array}{lr}\text { BIBLIOGRAPHY } & 141\end{array}$

$\begin{array}{lr}\text { List of Publications } & 153\end{array}$ 



\section{1.}

\section{INTRODUCTION}

$\mathrm{C}$

ALCUTTA 1895-one person must have grinned from ear to ear as the sound of a gunshot filled the air, followed by the rumbling of an exploding mine. This remarkable display did not announce the siege of Chitral, rather it was the contraption of J.C. Bose [46] who believed it was all necessary ${ }^{1}$ to demonstrate the abilities of his $5 \mathrm{~mm}(60 \mathrm{GHz})$ electromagnetic waves apparatus. Although millimeter waves (mm-waves) are usually associated with the next generation $(5 \mathrm{G})$ of wireless communications, the experiments of Bose predate even Marconi's telegraph. Still, examples of practical applications in the $60 \mathrm{GHz}$ frequency range are not abundant. This will likely change in the near future.

Today, the $2.4 \mathrm{GHz}$ and $5 \mathrm{GHz}$ Wi-Fi definitions have been the de facto standard of short-range wireless communications for over a decade [8, 61]. Their capacity continues to be expanded even while both frequency bands are being overused heavily [8]. This cannot continue indefinitely. Many readers will be familiar with the interference (performance) issues that arise when yet another neighbor deploys a new Wi-Fi access point in the neighborhood. One solution to this kind of problems is overpowering their signals by installing, e.g., the Engenius ECB-350o long range access point ${ }^{2}$. However, such a solution is neither socially responsible nor sustainable. A more thoughtful solution can be found in the mm-waves of Bose.

As these mm-waves are blocked by solid materials [82], and offer a tremendous bandwidth $[67,68]$ at the same time, they are ideal for a new generation of local broadband networks in homes and offices. A $60 \mathrm{GHz}$ signal will not propagate through walls, thus it will not interfere with the ongoing communications in neighboring rooms or buildings. Furthermore, governmental regulation agencies have set aside enormous amounts of (unlicensed) mm-wave spectrum around $60 \mathrm{GHz}$ (figure 1.1). Strengthened by the consumer's desire for more bandwidth, the general consensus between industry experts is that $60 \mathrm{GHz}$ needs to make its debut soon [97].

\footnotetext{
${ }^{1}$ The author has to agree with $\mathrm{mr}$. Bose.

${ }^{2}$ The author has experimentally found this to work very well.
} 


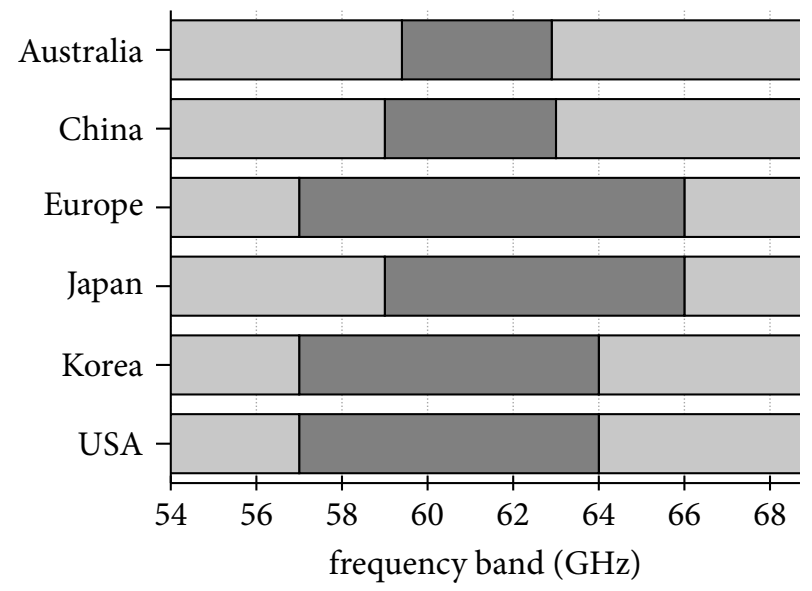

FIGURE 1.1 - Regional frequency allocation at $60 \mathrm{GHz}$. The dark colored spectrum has been reserved for unlicensed use, which should be ample for the next decade.

\section{1 $60 \mathrm{GHz}$ WIRELESS COMMUNICATION}

The transition to $60 \mathrm{GHz}$ for wireless communication is not trivial. Most of the spectrum allotment shown in figure 1.1 stems from the late 1990s. Ever since, it has mostly remained idle because of the technical difficulties and costs involved with the design and manufacturing of matching radio frequency (RF) circuitry. Although such problems are currently surmountable [97], there are still some issues that primarily relate to the propagation characteristics of mm-waves. For indoor situations, a predominant issues is path loss. According to the Friis transmission equation, these losses grow at least quadratically with the carrier frequency as the effective aperture of antennas typically scales with the wavelength [8]. Mitigation of these losses requires that the aperture is kept constant. A means to achieve this - that is both elegant and frequently quoted - is the utilization of antenna arrays, which aggregate the aperture of their elements.

\subsubsection{ANTENNA ARRAYS}

Like mm-waves, the idea of employing multiple antennas dates back to at least the early $20^{\text {th }}$ century. In 1905 , the German inventor K.F. Braun demonstrated with an arrangement of three antennas that he could reinforce transmission in one direction while diminishing radiation in other directions. This selectivity is the result of combining multiple transmitted signals, which is experienced as constructive interference in certain angles, while others experience destructive interference. The same principle also applies to receivers. Combining the signals received through multiple antennas improves the sensitivity in one direction while suppressing it in other directions. This spatial filtering is commonly referred to as beamforming [17][18] (although strictly speaking a beam is only formed while transmitting). An array is thus comparable to a dish- or horn antenna. 


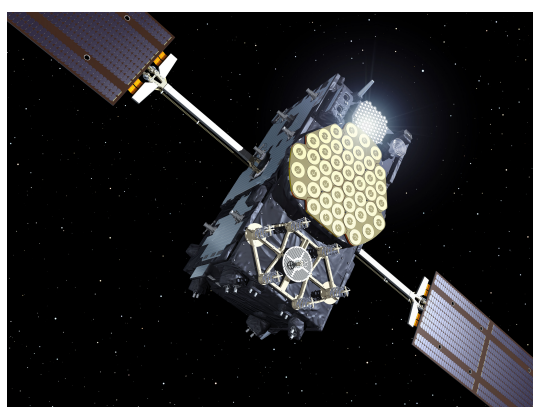

FIGURE 1.2 - Galileo-IOV

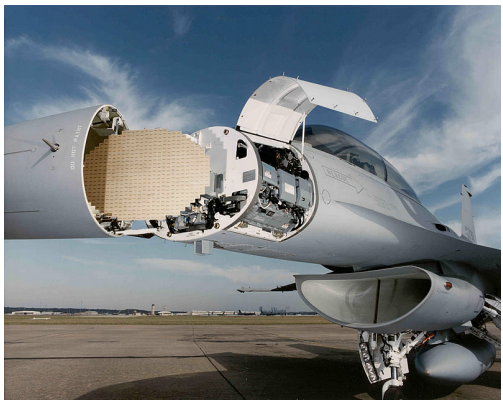

FIGURE 1.3 - F-16 AN/APG-68 radar

The advantage of antenna arrays is that their directionality can be controlled without the need for (bulky) mechanical steering devices. To redirect the beam of a dish antenna, its physical orientation must be altered accordingly. Arrays can be steered electronically by means of imposing appropriate delays to the individual channels (i.e., the signals received or transmitted through the different antenna elements). When this is controlled autonomously, to track for example moving targets, the array is called adaptive or smart [7].

Because of development costs, application of antenna array systems has historically been limited to areas where performance requirements take absolute priority. Typical examples are satellite broadcasting (figure 1.2), radar (figure 1.3) and radio astronomy. Within the consumer electronics market, beamforming is only seldom encountered. This will change when $60 \mathrm{GHz}$ becomes mainstream. Because of the associated high gain and directionality, beamforming is considered to be an enabling technology for $60 \mathrm{GHz}$ wireless communication. A first indication hereof is for example given by "Omnilink 6o", an adaptive beamforming system developed by SiBEAM [110], which has already been incorporated in the first chipsets to implement the $60 \mathrm{GHz}$ WirelessHD standard.

\subsubsection{OPTICAL-WIRELESS INTEGRATION}

Spectrum scarcity is not the only reason why $60 \mathrm{GHz}$ has gained interest. Higher frequencies also serve the purpose of providing more bandwidth. According to the accompanying standards such as IEEE 802.15.3C [67] and 802.11AD [68] (WiGig), $60 \mathrm{GHz}$ can potentially deliver transfer rates up to $7 \mathrm{Gbit} / \mathrm{s}$. This has given rise to the research performed on integration with fiber optical networks. Specifically, radio over fiber ( $\mathrm{RoF}$ ) [36] [76] has been identified as a flexible and cost-effective way to interconnect the $60 \mathrm{GHz}$ access points distributed throughout a building. The technique allows radio access control and signal processing to be performed at a centralized processing point, whilst radio signals are delivered (transparently) via optical fiber to a wireless access point. This results in benefits such as simplification of access points and reduced costs/complexity of the 'backbone' infrastructure [76]. 


\subsubsection{SOWICI}

Such an integration has also been defined in the smart optical wireless in-home communication infrastructure (SOWICI) project, the context in which the research described in this thesis has been carried out. This project, which was financed by the Netherlands Organization for Scientific Research (NWO), was initiated in the smart energy systems (SES) program which has the goal to make society's energy consumption more sustainable. Domestic appliances such as heating and lighting are often not adequately adjusted to actual needs. Considerable energy savings are expected from automation of appliances, to meet the specific needs of inhabitants. This relies on sensors and actuators that must be able to exchange information reliably and efficiently. SOWICI focuses on the infrastructure to support this.

The proposed network integrates this home automation with the next generation of high bit-rate data exchange. Use is made of the aforementioned RoF technique in combination with adaptive antenna arrays that act as wireless end-points. All communication is adaptively routed through a fiber backbone to room sized wireless pico-cells [36] that operate in the $60 \mathrm{GHz}$ frequency band. Within each room, the use of antenna arrays ensures that energy is only projected in the direction where it is needed, by means of beamforming. An impression of this infrastructure is provided in figure 1.4. It comprises a home communication controller (HCC), a fiber optical backbone and configurable radio access points (RAPs) which communicate with (mobile) wireless devices through highly directional beams. The HCC serves both as a bridge between the (external) access network and as the central processing point which takes care of routing, access control and beam steering. This thesis is mainly concerned with the tracking intelligence needed for correct beam steering, that must be controlled remotely from the HCC.

\section{Optical beamforming}

To integrate the wireless end-points seamlessly with the fiber optical backbone, beamforming is implemented using an integrated optical circuit. This means that, in order to change the direction of the beam, the appropriate (phase) delays are induced by optical components. There are several methods to impose a delay optically (the details are discussed in chapter 2). In SOWICI the choice was made to implement optical true time delays (OTTDs) [28], to support all the available bandwidth at $60 \mathrm{GHz}$ (figure 1.1). More specifically, it was decided to use switched delay lines. In this solution, only a finite set of delays is implemented from which a selection can be made during operation. In contrast to continuously tunable delays, the beam can therefore only be steered to a selected number of angles, but it also has the advantage of being much faster. For indoor scenarios this speed is important because the environment is prone to rapid changes with mobile devices involved (i.e., beam misalignment is more likely to occur when distances are short). To select the proper delay lines (and steering angle), SOWICI associates them with a particular photonic wavelength. By changing this wavelength from within the HCC, different delays will be selected such that the beam direction is changed remotely. 


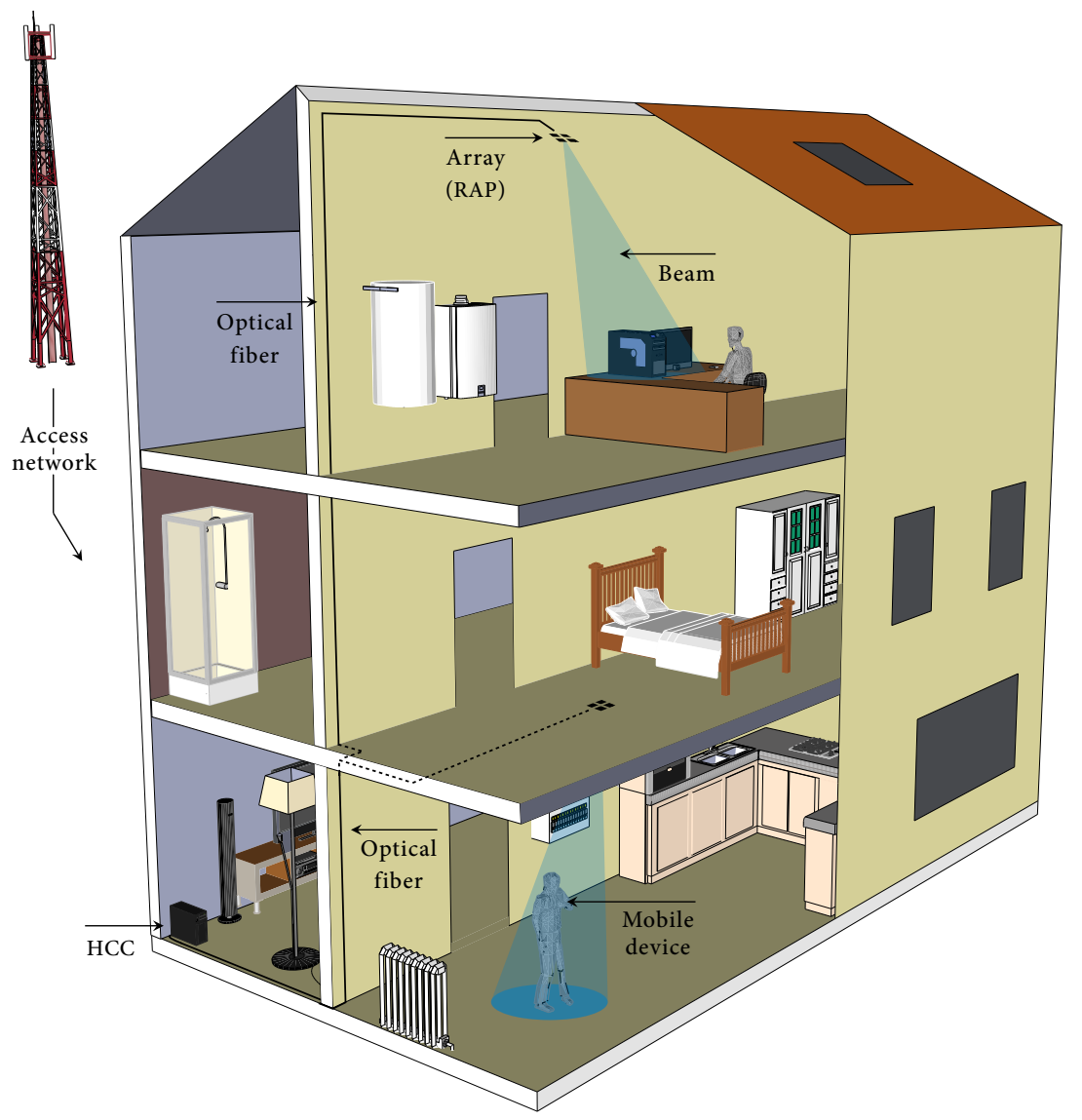

FIGURE 1.4 - Optical-wireless communications infrastructure (adaptation from [15])

\subsection{Problem STATEMENT}

Making a communication infrastructure with directional antennas is a nontrivial affair, because it changes many traditional aspects of wireless systems. Especially in the presence of mobile devices, establishing and maintaining links between an access point and its users becomes a challenge. Setting up connections requires localization of peer devices; maintaining a connection requires that their angular position relative to the array is tracked for beam steering purposes.

When an adaptive array is required, beamforming is often implemented digitally on the receiver side. Sampling each of the array's individual channels gives enormous flexibility (viz., cross-correlation of samples provides a wealth of knowledge about the environment). Consequently, the number of existing array based tracking and localization algorithms is abundant for the digital domain [7, 18]. Unfortunately, 
the same is not true for the analog domain. On the contrary, the all-analog type of beamformer is largely inflexible and options for adaptivity are very limited. In SOWICI, this inflexibility is exacerbated by the requirement of centralizing all processing in the HCC. Because the individual signals are summed optically (in the analog domain), before being conveyed over the fiber backbone, the HCC can only sample this combined output as a source for signal processing.

Essentially this limitation enforces that arrays continuously scan the entire room for the presence of wireless devices. A device will be located in the direction where the highest signal strength (i.e., measured power) is observed. In case of localization such an approach is considered acceptable. Comprehensive protocols have been developed [128] to assist in setting up wireless connections efficiently this way. For tracking of mobile devices, the use of scanning is inefficient in a communications scenario. Even when only the close vicinity of such a device's last known position is scanned, a substantial amount of steering takes place which does not directly contribute to a good communication link. Multiple scan angles have to be evaluated purely to see if the signal strength increases in that direction. During such a scan, the beam will not align with the position of the mobile device. This may be disruptive for the communications. Resulting losses in signal strength can lead to a high bit-error rate (BER) or even complete connection loss.

In summary, the problem statement of this thesis is formulated concisely as: development of an efficient array based tracking algorithm for communication purposes, that can cope with the limitation of all-analog beamforming.

\subsection{APPROACH}

As maintaining beam alignment is important for communications, the desired tracking algorithm should not have to steer unless the position of a mobile device requires it. This entails that the angle of incidence must be derived from the combined output of the analog beamformer. To achieve this, the tracking method that is proposed in this thesis monitors the power level of the output. Power is related to a particular angle by the shape of the beam, i.e. the beampattern, which in turn is a function of the array's geometry; and the amplification/attenuation and delay values that are applied to the beamformer's channels. Since the latter can be imposed from the HCC, the shape of the beampattern can be used to perform direction-of-arrival (DoA) estimation.

This approach has one problem, beampatterns are typically shaped symmetrically. A power measure will therefore be ambiguous in terms of the corresponding angle. The solution is found in utilizing asymmetrical beampatterns. These shapes are unconventional and require advanced algorithms to be synthesized. Given that shaped patterns used for tracking require additional specific features, the thesis will also focus on appropriate synthesis algorithm(s) and explain modifications where necessary. 


\subsection{Outhine}

The outline of the thesis is as follows. Chapter 2 provides a mathematical background and introduces the terminology for antenna array analysis and synthesis. In particular, relations for the shape of the beampattern are derived given the array's structure and a set of excitation values. It also covers various forms of optical beamforming and different adaptive array (tracking) algorithms, and discusses their compatibility. In chapter 3, synthesis of shaped beampatterns is explained for equispaced linear array structures. Pattern shapes suitable for tracking purposes will be synthesized (based on a 16-element linear array). The proposed tracking mechanism is described in chapter 4. This chapter also evaluates the algorithm's operation and performance through simulations. Chapter 5 details what steps are necessary for realization. These comprise modifications to the synthesis algorithm, to compensate the non-isotropic gain of realistic antenna elements, and optimization of amplification values so that these stay within realistic bounds. A final step is presented in chapter 6 . Herein, both synthesis and tracking are extended to planar arrays. Two-dimensional array structures increase directionality and are needed to cover an entire room. Conclusions are drawn in chapter 7 , followed by a summary of contributions and the recommended directions for future research.

\subsection{Conventions}

Mathematical conventions and antenna array terminology are mostly based on [7],[43] and [123]. Typically a scalar will be denoted in lowercase (e.g., $x$ ), a vector in lowercase bold face (e.g., $\mathbf{x}$ ) and matrices by uppercase bold face (e.g., $\mathbf{X}$ ). Exceptions to this convention will be explicitly stated. Updating or modifying a particular value is for example written as $x=\stackrel{x}{x}+1$. An overview of the meaning of symbols used throughout the thesis can be found in the nomenclature. 



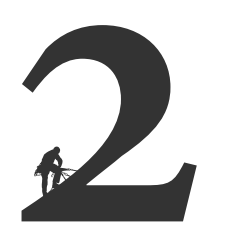

\title{
ANTENNA ARRAY PRINCIPLES AND RELATED WORK
}

\begin{abstract}
This chapter covers related work and provides the mathematical fundamentals of antenna arrays that need to be understood for the algorithms presented in later chapters. First, expressions for the response of a given array structure are formulated. Subsequently, common beampattern parameters and associated terminology are introduced. The second part of the chapter gives a brief overview of the research that has already been conducted in the fields of optical-and (all-analog) adaptive beamforming. This concerns selected methods to achieve beamforming in the optical domain, and algorithmic approaches to deal with a dynamic environment.
\end{abstract}

I NVESTIGATING a tracking solution based on manipulating the shape of the beampattern, as mentioned briefly at the end of the introduction chapter, requires that the beampattern is understood thoroughly. Aspects such as how the array geometry defines the shape of the beampattern and in what ways the beampattern can be altered will be explained in $\$ 2.1$. The notations in this section generally follow [123], although some definitions are taken from [43] because they are more fitting to the advanced subjects that follow later.

In $\$ 2.2$, the focus will be on existing optical beamforming techniques. There are several methods to implement beamforming using optical devices, and they are not equally suitable for an adaptive solution. In a comparison, their advantages and in particular their limitations will be stated. A similar overview is given for tracking- and adaptive-array algorithms in \$2.3. From an algorithmic perspective, the SOWICI requirements (\$1.1.3) will turn out to be a limiting factor for a multitude of different approaches. Especially because the targeted beamforming structure is all-analog, compatible tracking methods are scarce. Conclusions will be drawn based on the compatibility of different tracking algorithms and optical beamformers in $\$ 2.4$. 


\subsection{ARRAY PRINCIPLES}

\subsubsection{Structure}

Design and analysis of antenna arrays typically starts by defining the array structure. Arrays are said to consist of $N$ antenna elements. For every $n^{\text {th }}$ element, the $3^{-}$ dimensional position in Cartesian coordinates can be represented by a vector

$$
\mathbf{p}_{n}=\left[\begin{array}{c}
x_{n} \\
y_{n} \\
z_{n}
\end{array}\right], \quad n=0,1, \ldots N-1
$$

Based on how the $x, y$ and $z$ components are chosen, the array falls into one of the following three categories:

» Linear arrays

»Planar arrays

»Volumetric arrays

Equispaced linear arrays, as depicted in figure 2.1, will be the only linear structure used in this thesis. They are distinguished from arbitrary linear arrays by the property of having their elements spaced uniformly by a distance $d$. Restricting the element positions to such a distribution provides mathematical advantages that will be exploited later. The convention adopted here is that the elements are placed along the $x$-axis. Thus, for an array to be linear it must hold that $y_{n}=0$ and $z_{n}=0$. In some literature this array structure is referred to as the uniform linear array (ULA) [123]. The main disadvantage of using linear arrays is that they can only create directionality in the $x z$-plane. The main disadvantage of using linear arrays is that they can only create directionality in the $x z$-plane.

Planar arrays are defined as arrays whose element positions are confined to the $x y$-plane (i.e., $z_{n}=0$ ). However, in most of the literature that covers planar arraysincluding this thesis-the element distribution exhibits some regularity. Often the elements are laid out on a grid pattern (not necessarily rectangular), as shown in figure 2.2. In chapter 6 of this thesis, two planar array structures will be discussed: one where the elements are laid out on a rectangular grid structure (figure 2.2); another places the elements along a diagonal lattice. Planar arrays create directionality in both the $x$ and $y$ direction.

Volumetric arrays are entirely generic. A subset of this array type, the conformal arrays, will sometimes arise from geometrical placement constraints such as the wing of an airplane or the hull of a ship. In general these array are difficult to cope with mathematically. Since the indoor scenario described in chapter 1 does not dictate the need for an array from this category, it will not be treated in detail here. 


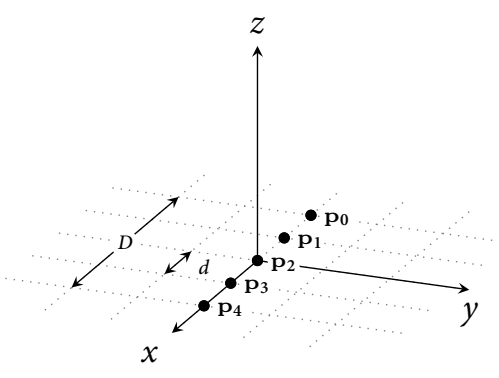

FIGURE 2.1 - Linear array

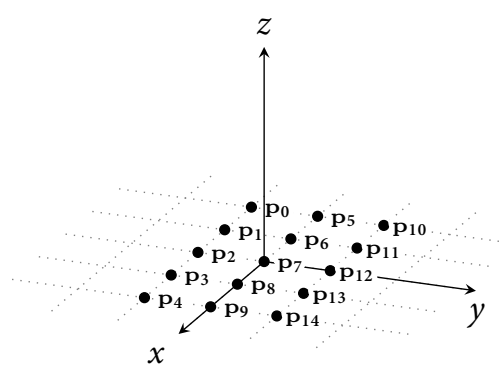

FIGURE 2.2 - Planar array

\subsubsection{RESPONSE}

When analyzing the response of an array to an external signal field, it shall be assumed that the far-field condition [117] holds. Under this condition, signals impinging the array may be considered as plane waves. In order to be in the far field region of an antenna array, a source has to be separated from the array by a distance $r$ that is much larger than the Fraunhofer distance

$$
d_{f}=\frac{2 D^{2}}{\lambda}
$$

In this expression, $D$ is the array's aperture (i.e., its largest dimension as indicated in figure 2.1) and $\lambda$ the signal's wavelength. Given the $5 \mathrm{~mm}$ wavelength of a $60 \mathrm{GHz}$ signal, and the fact that the inter-element spacing $d$ will not exceed $1 / 2 \lambda$, it is evident that this condition will be easily fulfilled.

The array's response is evaluated in the spherical azimuth $(\phi)$ and elevation $(\theta)$ coordinate system, which has been visualized in figure 2.3. Azimuth angles will be defined relative to the positive $x$-axis and the elevation angle relative to the positive $z$-axis (zenith). Because $r \gg D$, the angle of incidence of the plane wave can be represented as a unit vector $\mathbf{u}$ perpendicular to this plane. As the array topography (eq. 2.1) is defined in Cartesian coordinates, $\mathbf{u}$ must be written as

$$
\mathbf{u}=\left[\begin{array}{l}
u_{x} \\
u_{y} \\
u_{z}
\end{array}\right]=\left[\begin{array}{c}
\sin \theta \cos \phi \\
\sin \theta \sin \phi \\
\cos \theta
\end{array}\right]
$$

Due to the physical separation of the antenna elements, they will be affected by the plane wave at different points in time. For example in figure 2.3, the plane arrives at $\mathbf{p}_{\mathbf{2}}$ first, then $\mathbf{p}_{\mathbf{0}}$ followed by $\mathbf{p}_{3}$, and finally $\mathbf{p}_{\mathbf{1}}$. The relative time delays between arrival are a function of the position $\mathbf{p}_{\mathbf{n}}$, the angle of incidence $\mathbf{u}$ and the wave's propagation speed $c$. When dealing with electromagnetic (EM) signals, $c$ 


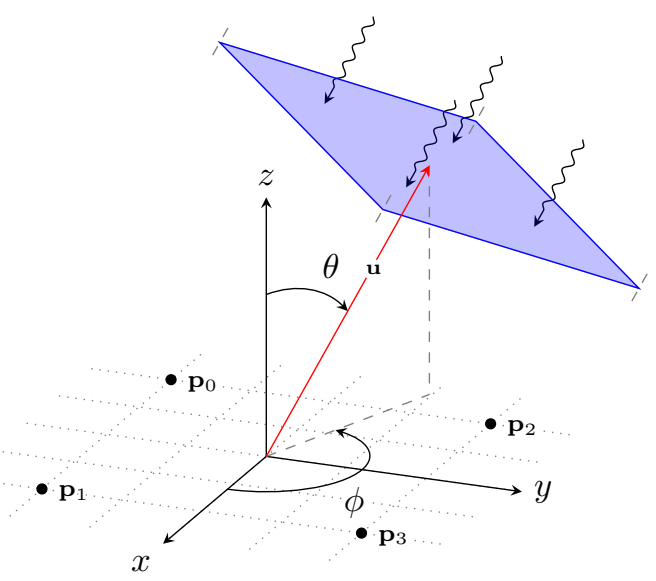

FIGURE 2.3 - Plane wave impinging a 4-element planar array from $\phi=112^{\circ}, \theta=20^{\circ}$

corresponds to the speed of light. Then for the $n^{\text {th }}$ element, the time delay is given by

$$
\check{\tau}_{n}=\frac{\mathbf{u}^{T} \mathbf{p}_{\mathbf{n}}}{c}
$$

For a given point in time $t$, the use of multiple antennas gives rise to a collection of signals $x\left(t, \mathbf{p}_{n}\right)$ observed at the element positions $\mathbf{p}$. This may be written as

$$
\mathbf{x}(t, \mathbf{p})=\left[\begin{array}{c}
x\left(t-\check{\tau}_{0}\right) \\
x\left(t-\check{\tau}_{1}\right) \\
\vdots \\
x\left(t-\check{\tau}_{N-1}\right)
\end{array}\right]
$$

Classical antenna array theory, which shall be used extensively, prefers to use the notion of phase rather than time. To this end, the frequency wavenumber $k$ is introduced. When a plane wave travels through a homogeneous medium, $k$ expresses the number of radians per unit distance. The corresponding wave vector $\mathbf{k}$ is defined as

$$
\mathbf{k}=\frac{\omega}{c} \mathbf{u}=\frac{2 \pi}{\lambda} \mathbf{u}=k \mathbf{u}
$$

where $\omega$ is the operating frequency. In terms of $\omega$, the $n^{\text {th }}$ component from eq. 2.5 can be denoted by

$$
X_{n}(\omega)=X(\omega) e^{-j \omega \check{\tau}_{n}}
$$


due to the Fourier transform. By realizing that

$$
\omega \check{\tau}_{n}=\mathbf{k}^{T} \mathbf{p}_{n}
$$

it is possible to write

$$
\mathbf{X}(\omega)=X(\omega) e^{-j \mathbf{k}^{T} \mathbf{p}_{n}}=X(\omega) \mathbf{v}_{\mathbf{k}}
$$

The complex exponential vector $\mathbf{v}_{\mathbf{k}}$ in this equation is sometimes referred to as the array manifold vector [123], and describes all spatial characteristics of the antenna array. Without any processing, the output of the array is the sum of the manifold vector elements.

\subsubsection{BEAMFORMING}

Depending on the angle of incidence, summation causes constructive or destructive interference in $\mathbf{x}$, giving the array its directionality. To control this directionality, a beamforming network can be placed between the antenna element and the output $y(t)$ of the array. This has been illustrated schematically in figure 2.4. A beamformer could for example shift the inputs, such that they align in time before being combined. How this operation should be implemented depends on the bandwidth of the signal with respect to its carrier frequency. For a narrowband signal [7], phase shifts $\left(\varphi_{n}\right)$ would suffice, whereas wideband signals require true time delays $\left(\tau_{n}\right)$ or time delay approximation methods. A neatly formulated overview of the latter can be found in [21]. Each channel can also be given a different amplitude $\left(a_{n}\right)$. Amplitude tapering will give even more control over the response of the array. In terms of relative phase differences, a combination of the two is denoted as

$$
I_{n}=a_{n} e^{j \phi_{n}}
$$

so that it can conveniently be represented as a multiplication with the complex conjugate $I_{n}^{*}$ in eq. 2.9 [123]. In the time domain, the response (to a plane wave) of the array manifold vector and beamformer together can then be written as

$$
y(t)=\mathbf{I}^{H} \mathbf{v}_{\mathbf{k}} e^{j \omega t}
$$

where the complex I values $\left({ }^{H}\right.$ denoting the hermitian transpose) are called either weights [123] or excitations [43]. The more traditional term (current) excitation is adopted here, which explains why $\mathbf{I}$ is capitalized despite it being a vector.

Note-While this thesis is aimed at (optical) time delay beamformers, the next few chapters primarily reason in phase differences. This is due to the fact that array pattern synthesis, which is used to control the shape of the beampattern (discussed in chapter 3 ), follows the narrowband model. In chapter 5 , the accumulated relative 


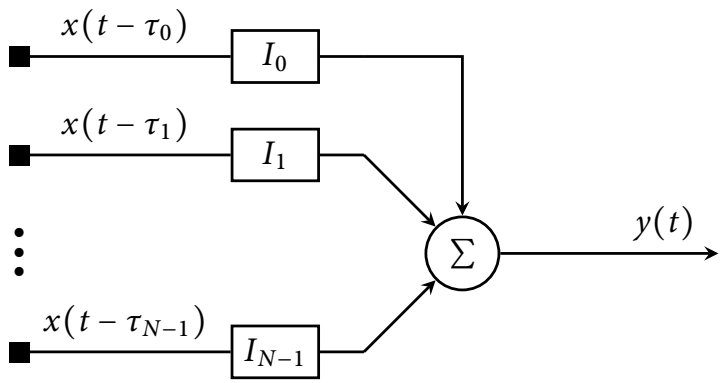

FIGURE 2.4 - Beamformer

phase differences in terms of $\mathbf{k}$ will be converted back to time delays and applied to the appropriate expressions to (re)evaluate the array response.

\subsubsection{BEAMPATTERN}

In eq. 2.11, the combined effect of complex excitations and the array manifold

$$
\mathbf{I}^{H} \mathbf{v}_{\mathbf{k}}
$$

is termed the frequency-wavenumber response function. When evaluated against the angle of incidence, it gives the array's beampattern

$$
B(\phi, \theta)=\mathbf{I}^{H} \mathbf{v}_{\mathbf{k}}(\phi, \theta)
$$

The performance of an array (viz., the advantage over a single antenna) is usually expressed through this function. As such, it will have a prominent role in this thesis. The properties of a beampattern from linear arrays are discussed first.

\section{Linear array patterns}

In anticipation of the subject discussed in the subsequent chapter, the compact notation of eq. 2.13 is preferably written out for better agreement with synthesis literature

$$
\begin{aligned}
B(\phi, \theta) & =\mathbf{I}^{H} \mathbf{v}_{\mathbf{k}}(\phi, \theta) \\
& =\sum_{n=0}^{N-1} I_{n} e^{j \mathbf{k}^{T}(\phi, \theta) \mathbf{p}_{n}}
\end{aligned}
$$

Now consider again the equispaced linear array as shown in figure 2.1. Due to the uniform spacing $d$, the position of the $n^{\text {th }}$ element can expressed by 


$$
p_{n}=x_{n}=\left(n-\frac{N-1}{2}\right) d, \quad n=0,1, \ldots, N-1
$$

as $y_{n}=z_{n}=0$. It is also customary to evaluate the beampattern of a linear array solely in the $x z$-plane, because it does not create directionality in the orthogonal dimension. This means that $\phi=0$ and therefore the manifold of a linear array simplifies to

$$
\mathbf{V}_{\mathbf{k}}=e^{-j \mathbf{k}^{T}(\phi, \theta) \mathbf{p}_{n}}=e^{-j \frac{2 \pi}{\lambda} \sin (\theta) x_{n}}=e^{-j\left(n-\frac{N-1}{2}\right) k d \sin (\theta)}
$$

with $k=|\mathbf{k}|=2 \pi / \lambda$. The beampattern can now be written as

$$
B(\theta)=\Phi \sum_{n=0}^{N-1} I_{n} e^{j n k d \sin (\theta)}
$$

where

$$
\Phi=e^{-j\left(\frac{N-1}{2}\right) k d \sin (\theta)}
$$

represents a constant phase shift that is usually suppressed from eq. 2.17 in the far field. As $B(\theta)$ is a complex function, it contains a phase (i.e., the argument $\angle B(\theta)$ ) and a magnitude $(|B(\theta)|)$. Although the phase could also be plotted against various incidence angles, in the far field one is typically only interested in the magnitude of the beampattern. Plotting the magnitude in polar coordinates can be seen in figure 2.1, for $N=8, d=1 / 2 \lambda$ and $I_{n}=1 / N$. Constructive interference gives rise to the beam (or main lobe) seen at $\theta=0^{\circ}$. This is what the beamformer (i.e., the array and excitations combined) owes its name to. In array terminology, $0^{\circ}$ is the broadside angle, similar to the boresight angle of a singular antenna. Although polar coordinates and magnitude give the most faithful representation of the physical intensity

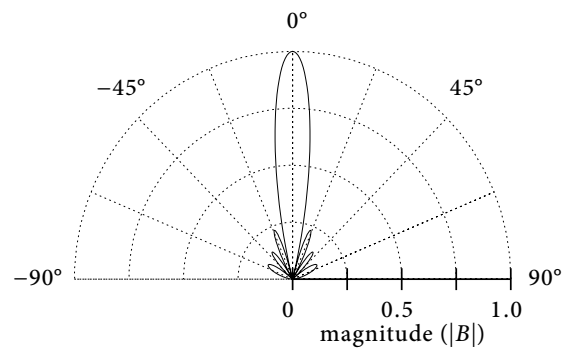

FIGURE 2.5 - Polar beampattern plot

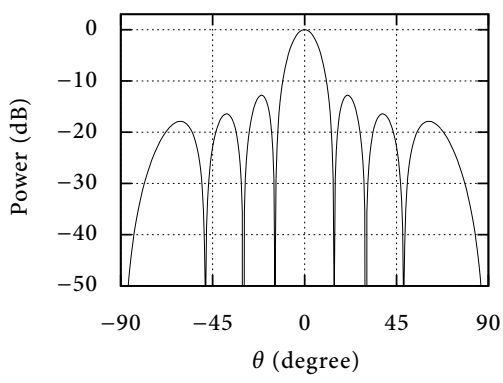

FIGURE 2.6 - Standard representation 
(i.e., power) of the pattern, beampatterns are often plotted in a logarithmic scale with the $\theta$ angle 'rolled out' along the horizontal axis. As seen in figure 2.2, certain details of the beampattern can be recognized easier in this form. Besides a main lobe, several smaller lobes can be distinguished. These sidelobes are interspersed by deep nulls. Nulls originate from destructive interference in the same way that lobes arise from constructive interference.

Note that eq. 2.17 is defined for $[-\infty, \infty]$, but only plotted for $-90^{\circ} \leq \theta \leq 90^{\circ}$. This is due to physical constraints that apply to a (ceiling) mounted array. If $B(\theta)$ were plotted beyond this physical range, it would be found that it is periodic with respect to $k d$ [123]. In literature, this period is sometimes referred to as the array's visible region. The $1 / 2 \lambda$ spacing chosen for figure 2.2 can be considered ideal. When substituted in $k d$, it yields a period of $\pi$ that matches exactly with the $180^{\circ}$ physical range of the array. If $d$ would be less than $1 / 2 \lambda$, the period of $B(\theta)$ exceeds the physical range. This will be experienced as broadening of the beam, that can be seen for $d=1 / 4 \lambda$ in figure 2.7. On the other hand, when $d>1 / 2 \lambda$, the shape of the beampattern (partially) repeats itself. The latter is be referred to as spatial aliasing, analogous to the spectral aliases found for an undersampled time signal. The effect can be seen in figure 2.8 for $d=\lambda$.

Assessment of the performance of a particular beampattern requires quantifiable metrics. To this end, some of the more commonly used parameters [7] to describe a beampattern are

» half-power beamwidth (HPBW)

" sidelobe level (SLL)

» inter-null beamwidth (INBW)

Each of the above has been visually indicated in figure 2.9. In this example, the depicted $1 / 2 \lambda$ spaced 8 -element beampattern features a HPBW of $13^{\circ}$, an INBW of $29^{\circ}$ and the shown SLL is $-12.8 \mathrm{~dB}$. Note that HPBW is primarily meaningful for a symmetric beam. As many beampatterns presented later do not adhere this requirement, the INBW shall be used to express beamwidth. Furthermore, the SLL

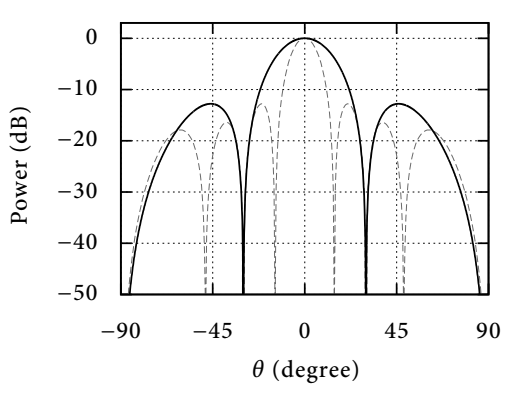

FIGURE 2.7 - Beampattern : $1 / 4 \lambda$ spacing

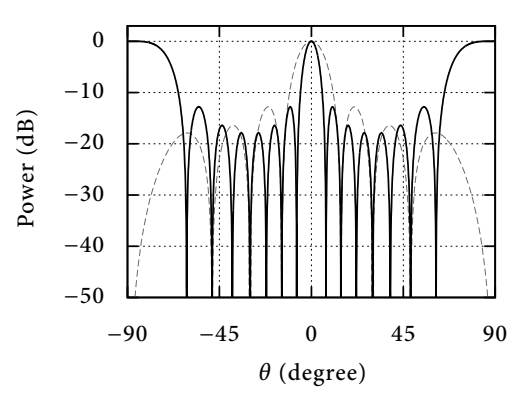

FIGURE 2.8 - Beampattern : $\lambda$ spacing 
depends on the particular sidelobe chosen to be quantified. Typically this will be the highest one (i.e., adjacent to the main lobe) or a root-mean-square (RMS) SLL is defined [113]. For the majority of the pattern shapes to be presented, the SLL will be either invariant or individually specified.

In order to compare two beampatterns in terms of the performance, measures such as the array gain and directivity are needed. Directivity $(\Gamma)$ represents the maximum (radiated) intensity divided by the average intensity [123]. Generally this means narrower beams and (on average) lower sidelobes are pursued. The definition of array gain requires a more elaborate theoretical background [123] that will not be presented here.

\section{Planar array patterns}

Finding an expression for the beampattern of a planar array follows the linear case analogously. The difference is that, since $y_{n}$ will not necessarily be 0 , the manifold vector becomes somewhat more comprehensive. In generic form, the response of a planar array is written as

$$
B(\phi, \theta)=\sum_{n=0}^{N-1} I_{n} e^{j k\left(x_{n} \sin (\theta) \cos (\phi)+y_{n} \sin (\theta) \sin (\phi)\right)}
$$

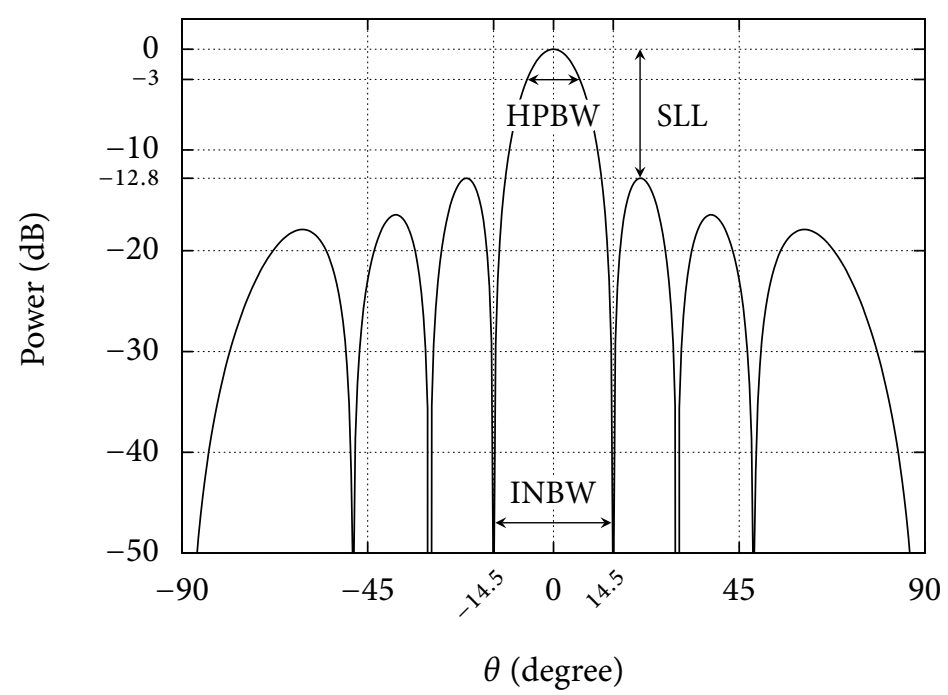

FIGURE 2.9 - Beampattern parameters 
Chapter 6 will tailor this relation to the specific grid array geometries that are used. Their response is principally evaluated in full $(\phi, \theta)$-space, as per figure 2.3. However, for detailed analysis it can be more insightful to show only the $\theta$ dependency of one particular $\phi$ angle. This is referred to as a $\phi$-cut.

\section{Element factor}

A detail that has been omitted for mathematical clarity is the gain of the individual antenna elements. The expressions shown up to this point all assume that the array consists of isotropic radiators/sensors. In practice, each element in the array [6] will have its own directional pattern $E_{n}(\theta)$, called the element factor. The actual beampattern is therefore a combination of $E_{n}(\phi, \theta)$ and eq. 2.17

$$
B(\phi, \theta)=\sum_{n=0}^{N-1} E_{n}(\phi, \theta) I_{n} e^{j \mathbf{k}^{T}(\phi, \theta) \mathbf{p}_{n}}
$$

From here on, the beampattern without the element factor shall therefore distinctively be denoted as the array factor $F(\phi, \theta)$. Under the assumption that the element factor is the same for each $n$, the subject can be deferred until chapter 5 . The element factor will be considered in more detail there, because its effect on the shape of a steered beampattern cannot be neglected.

\subsubsection{BEAM STEERING}

Every pattern shown thus far is an example of conventional beamforming. In many applications, especially communications within a dynamic environment such as the one described in chapter 1, it will be desirable to control the directionality of the array [55]. The prime example hereof is beam steering. Although mechanical steering of the main lobe (i.e., positioning the array element perpendicular to a desired angle) is a possibility, the more common approach with antenna arrays is by adjusting the excitations. To align the pattern's main response axis (MRA) with the desired steering- or scan angle $\theta_{d}$, the excitations should be assigned the values

$$
I_{n}=e^{-j n k d \sin \left(\theta_{d}\right)}
$$

The result of applying $\theta_{d}=-22.5^{\circ}$ steering to an 8 -element ULA can be seen below

Often when a beamformer is capable of steering, the (theoretical) maximum steer angles of $\pm 90^{\circ}$ are referred to as positive or negative endfire. Steering of nulls [124] is sometimes also considered to be part of an adaptive array. However, in spite of this capability being very useful for communications, it will not be a major topic in this thesis. The reason is that switched beamforming does not support this feature well from an implementation perspective (\$2.3 will elaborate further on this subject). 


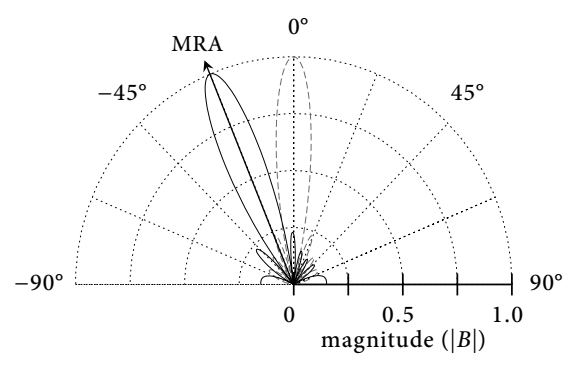

FiguRE 2.10 - Beam steering

\subsection{OPTICAL/ANALOG BEAMFORMING}

All-optical implementation of the beamforming network has been a topic of interest since 1972 [85]. Consequently, a wide variety of methods have been developed to achieve this. Comprehensive surveys of existing alternatives are available from literature $[30,83,84]$. In this thesis, the physical realization of the (optical) beamformer can mostly be observed as a 'black box'. The techniques that are developed are principally independent of the type of beamformer ${ }^{1,2}$. Still, as the envisaged tracking method demands certain capabilities, some methods of excitation realization are impractical and it will be useful to know which ones.

In general one can distinguish switched beamformers from continuously tunable beamformers. A beamformer that is continuously tunable, such as for example in [34] and [108], enables real-time synthesis of an optimal beampattern that can be excited by tuning the $I_{n}$ values as calculated. Of course, only within realistic bounds and with a given accuracy. For a switched beamformer, tunability is limited to discrete values. Accordingly, only a finite set of beampatterns can be generated. The required excitation values $I_{n}$ need to be defined a priory, as they become fixed during the manufacturing process of the beamformer. In most cases, a tunable beamformer will perform better because it allows more fine grained steering of the MRA. On the other hand, switched beamformers are typically much simpler and therefore likely to be considered first in a commercial product $[94,106]$.

Further sub-categorization is based on how the $I_{n}$ values are implemented. This can be based on the amplitude $\left|I_{n}\right|$, but the more commonly chosen component is the argument of $I_{n}$. In the narrowband case [7], (optical) phase shifters [58] can be employed to compensate the delays caused by path length differences between the array elements (eq. 2.4). This has long been the primary choice of controlling the scan angle of the beam, which has also lead to the term phased array antenna. However, because approximating a time delay by a phase shift will only be exact for one particular frequency, phase shifters make the shape of the beam dependent

\footnotetext{
${ }^{1}$ Although they will be most interesting for the all-analog kind, and likely suboptimal for others.

${ }^{2}$ Type refers to the analog or digital domain, optical or microwave etc.
} 
on the operating frequency. This can have a negative effect on the performance of the array. In literature this problem is referred to as beam squint. Especially in the optical domain, the use of true time delay is much more popular for this reason.

Down to the physical realization level of optical true time delays, the list of alternatives is extensive (e.g., [30, 50, 65, 116, 136]). While the precise details do not matter here, two more properties need to be mentioned before considering adaptive arrays. First, in a conventional time delay beamformer, the (linear) compensation delays $\tau_{n}$ for a $\theta_{d}$-steered beampattern are given by $[80]$

$$
\tau_{n}=n \frac{d}{c} \sin \left(\theta_{d}\right), \quad n=0, \ldots, N-1
$$

Various photonic beamformers exploit the regularity of eq. $\mathbf{2 . 2 2}$ by making use of cascaded optical delays (e.g., $[29,87]$ ). Unless the delays are continuously tunable [108], such a structure limits the $\tau_{n}$ differences to integer multiples of a particular chosen minimum delay. Imposing arbitrary delays (chapter 5) will be highly impractical. The second consideration is the rate at which the excitation values can be adjusted. This determines how fast the shape of the beampattern can be updated which is important when servicing multiple wireless peer devices in a time division multiple access (TDMA) fashion, or when mobile devices to be tracked exhibit highly dynamic behavior (chapter 4 ). In case of a continuously tunable delay, such as the heated ring resonator solution presented in [135], adjusting the beampattern will require roughly one millisecond. For in-door situations, where the distance to the array will be relatively small, this is noteworthy. Switchable matrices of hardwired true time delays (e.g., [94]) have been shown to achieve sub-microsecond speeds [98]. This is more than sufficient in the context of the infrastructure presented in chapter 1.

Note-Driven by the aforementioned advantages and drawbacks, it was decided during the early SOWICI project discussions that a beamforming network would be employed that is both switched and makes use of true time delays [28]. Although a particular type of implementation shall not be enforced, some parts of the thesis will reflect that decision, most notably in chapter 4 .

\subsection{ADAPTIVE ARRAYS}

An array is considered to be adaptive if it is able to dynamically adjust its excitations to respond to changing signal conditions. This may be a change in direction of the impinging signal, but it could also mean the presence of interference. In this section, the algorithms that govern optimal excitation values for a given time instant are reviewed. What defines optimal depends on whether the underlying beamforming structure is switched or continuously tunable. An interesting categorization hereof is provided in [7], which will also be used here. Due to the discrete nature of a switched beamformer, it will be less versatile and is said to be partially adaptive. When the beamformer is continuously tunable, it could for example simultaneously 


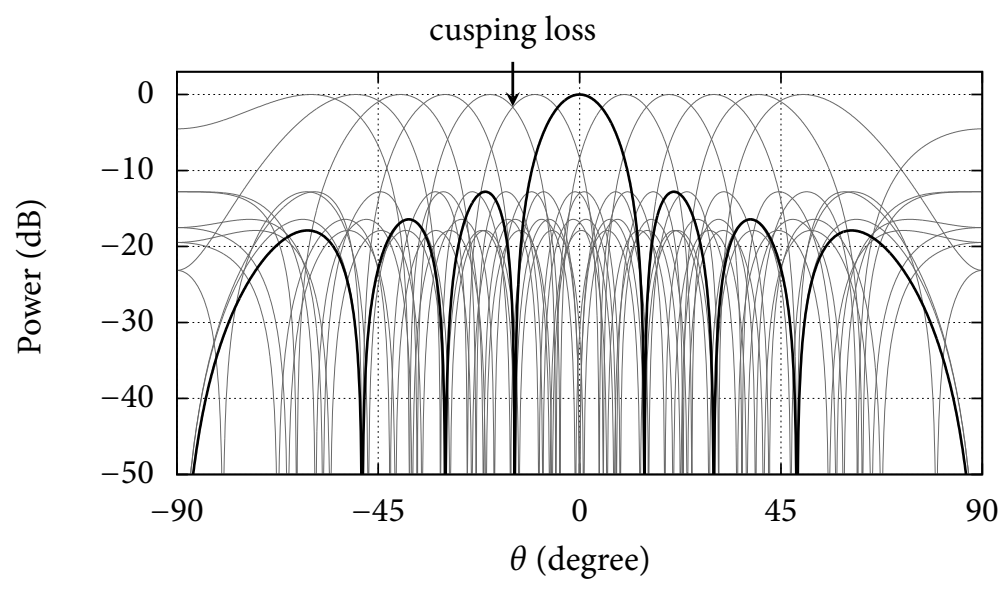

FIGURE 2.11 - Scanning a $120^{\circ}$ sector every $10^{\circ}$

place a null in the direction of interference while steering the MRA to the angle of interest. The class of algorithms that makes use of tunable excitation to the full extent is called fully adaptive.

\subsubsection{Partially adaptive}

Partially adaptive beamformers either are scanning or switched-beam arrays. Applications such as radar and sonar require that large angular sectors are observed to acquire possible targets. Scanning means that a sector is distributed over a number of predefined beams, as illustrated in figure 2.11. While scanning, the beamformer probes different (predefined) scan angles, by updating the excitation values according to a programmed sequence. These values could for example be stored in a memory or they could be hardwired. Scanning should strictly not be seen as an adaptive array algorithm, however, it works well for localization purposes using a switched beamformer (\$1.1.3). Once a target has been acquired, a different kind of switched beamforming can be used to track it. One could for example narrow the sector down to three beams (dynamically), the one which acquired the target and the two adjacent ones. Note the inherently associated cusping loss [7] shown in figure 2.11. This is another reason why switched beamformers are called partially adaptive. Classical examples of switched arrays are the Buttler matrix [59] and the Rotman lens [9]. In the high frequency and optical context, switched beamformers are fairly common $[88,94,119]$.

\subsubsection{Fully ADAPTIVE}

When no restrictions apply to the excitation values, the more advanced fully adaptive array techniques can be considered. Herein, the excitation values are continu- 
ously updated based on a temporal reference or spatial reference. Alternatively, blind beamforming is capable of achieving similar results by exploiting other structural or statistical properties of the expected signal.

Temporal reference beamforming refers to the use of training sequences to create a known reference. An optimal set of excitations $\mathbf{I}_{\text {opt }}$ is defined by the Wiener-Hopf optimum [7]. From the temporal reference, this optimum can be computed by

$$
\mathbf{I}_{\mathrm{opt}}=\mathbf{R}_{x x}^{-1} \boldsymbol{\gamma}_{r x}
$$

In eq. 2.23, $\mathbf{R}_{x x}^{-1}$ is the spatial covariance matrix (correlation between signals from different array elements) and $\gamma_{r x}$ cross-correlation vector between the $\mathbf{x}_{n}$ signals received at the elements and reference signal $r$. When $E[\cdot]$ signifies the expected value and $K$ the number of samples taken from the different elements, the spatial covariance matrix is computed by

$$
\mathbf{R}_{\mathbf{x x}}=E\left[\mathbf{x} \mathbf{x}^{H}\right]=\left[\begin{array}{cccc}
R_{11} & R_{12} & \cdots & R_{1 N} \\
R_{21} & & & \\
\vdots & & & \\
R_{N 1} & & \cdots & R_{N N}
\end{array}\right]
$$

with $R_{i j}$ given with

$$
R_{i j}=\frac{1}{K} \sum_{k=1}^{K} x_{i}[k] x_{j}[k]
$$

The $\gamma_{r x}$ vector is found by

$$
\boldsymbol{\gamma}_{r x}=E\left[r \mathbf{x}^{*}\right]=\frac{1}{K} \sum_{k=1}^{K} r[k] \mathbf{x}^{*}[k]
$$

Optimization of the output $y$ occurs based on criteria such as a minimum mean square error or minimum variance distortionless response [7]. Typically a number of estimations $\mathbf{R}_{x x}$ will be required for (asymptotic) convergence to the optimum. A very desirable property of temporal reference beamforming is that it automatically tracks the desired signal while canceling the $N-1$ strongest interferers. A strong drawback is the need for a training data, which is inserted as overhead in a communication system. Examples of temporal reference beamforming algorithms are minimum least squares or recursive least squares. For details one can refer to [55].

Spatial reference algorithms make use of the known spatial properties of the array geometry (\$2.1.1). The primary application of these algorithms is (high accuracy) DoA estimation of impinging signals, which typically translates to a result where 
DoAs are related to a particular power level. In the basis, a result is always obtained by considering the output $y=\mathbf{I}^{H} \mathbf{x}$ of the beamformer as shown in figure 2.4. When the power based on a particular set of excitation values is to be found, this can be expressed as

$$
\begin{aligned}
P & =E\left[|y|^{2}\right] \\
& =E\left[\left|\mathbf{I}^{H} \mathbf{x}\right|^{2}\right] \\
& =\mathbf{I}^{H} E\left[\mathbf{x} \mathbf{x}^{H}\right] \mathbf{I} \\
& =\mathbf{I}^{H} \mathbf{R}_{\mathbf{x x}} \mathbf{I}
\end{aligned}
$$

in which the covariance matrix $\mathbf{R}_{\mathbf{x x}}$ can be recognized. The simplest forms of spatial reference algorithms evaluate eq. 2.27 with different $\mathbf{I}\left(\theta_{d}\right)$ (eq. 2.21), thus performing a virtual scan over the entire visible region. Depending on the conditions, the granularity of the chosen $\theta_{d}$ angles, and above all the accuracy of the algorithm used, the DoA of the desired signal will likely be found at the peak power. Examples of more advanced DoA estimation techniques are

» Capon's minimum variance [31]

" Maximum Likelyhood Estimation (MLE) [49]

" Multiple Signal Classification (MUSIC) [109]

»Estimation of Signal Parameters by Rotational Invariance Techniques (ESPRIT) [92]

In extension of their result, a pattern can be synthesized that directs the MRA to the desired signal while simultaneously placing nulls at the strongest interferers.

Blind beamforming requires neither a training sequence nor information about the array structure. Instead, the excitations are updated based on the statistical (e.g., self-coherence) or structural (e.g., constant modulus) properties of an expected signal. To give an example, the constant modulus algorithm (CMA) [112, 134] has been designed to exploit the constant envelope of phase-shift keying (PSK) signals. These types of signals would exhibit a constant amplitude $y_{0}^{2}$ if channel distortions did not cause it to fluctuate. CMA minimizes a cost function $J$, that is defined as the expected deviation of the squared modulus of the beamformer output with respect to the constant modulus

$$
\begin{aligned}
J[k] & =\frac{1}{2} E\left[\left(|y[k]|^{2}-y_{0}^{2}\right)^{2}\right] \\
& \left.=\frac{1}{2} E\left[\left|\mathbf{I}^{H} \mathbf{x}[k]\right|^{2}-y_{0}^{2}\right)^{2}\right]
\end{aligned}
$$


Minimization of the cost function is often attained iteratively by calculating a gradient vector $\nabla_{\mathrm{I}} J$ with respect to $J$ [21], which is in turn used to update the excitation vector in the opposite direction

$$
\mathbf{I}[k+1]=\mathbf{I}[k]-\mu \nabla_{\mathbf{I}} J
$$

The $\mu$ parameter is called the step-size and determines the convergence rate [7]. An optimal output is reached when the cost function is reduced to zero and the deviation from the constant modulus have therewith been compensated by the excitation values. CMA comes in various flavors $[56,122]$ and can be modified to support different forms of PSK modulation [21]. Another example of blind beamforming is SCORE [2], which makes use of cyclostationarity (spectral self-coherence).

\subsubsection{COMPATIBILITY AND APPROACH}

Having reviewed a wide variety of the more advanced (fully) adaptive beamforming algorithms, it should be clear that this class relies extensively on the availability of data sampled at the individual array channels $(\mathbf{x}[k])$. For an all-analog beamformer, such as the optical variant discussed in \$2.2, this will not be the case. Only the combined output of the beamformer $y[k]$ can be used for signal processing. A covariance matrix can therefore not be constructed, unless the amplitude of every element but one is asserted zero, in order to reconstruct $\mathbf{x}$ by sampling the elements sequentially $^{3}$. A similar strategy is reported in [118] for the MUSIC algorithm. However, this will not be considered here because it entails a high penalty within a high bit-rate communication system.

When literature is consulted for adaptive techniques specific to single-port analog beamformers, references are not abundant. Although there is some variation, essentially all the work that focuses on this niche uses a technique that is called applying (orthogonal) perturbation sequences $[27,130]$. The general idea is similar to that of CMA (eq. 2.28), but the gradient is replaced by one $\left(\nabla_{\mathbf{V}}\right)$ that is defined in terms of output power with respect to the control voltages of the (phase) excitations. As the power is a function of these voltages, the latter are perturbed to maximize the output using for example a steepest-descent method [70]. Noteworthy variants hereof are [47]; the work carried out at the University of Waterloo [70], which applies it in the context of an optical beamformer; and [37], which presents a modified version of the CMA algorithm (M-CMA) based on perturbations. Granted that it has been shown to work in the optical domain, and that there is little alternative, this method also has some considerable drawbacks. For example, it requires continuously tunable phase/time shifters; characterization of the relation between the voltage sources and the output demands profound calibration; but most of all, it does not match with the optical wavelength controlled steering proposed in \$1.1.3. This thesis aims for a more generic solution, that will also support switched true time delay beamformers.

\footnotetext{
${ }^{3}$ Provided that the signal is sufficiently invariant with respect to the time needed.
} 


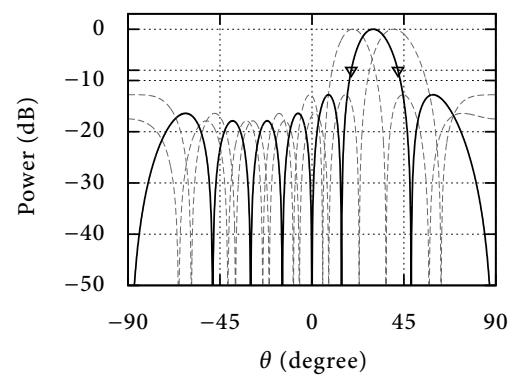

FIGURE 2.12 - Symmetry ambiguity

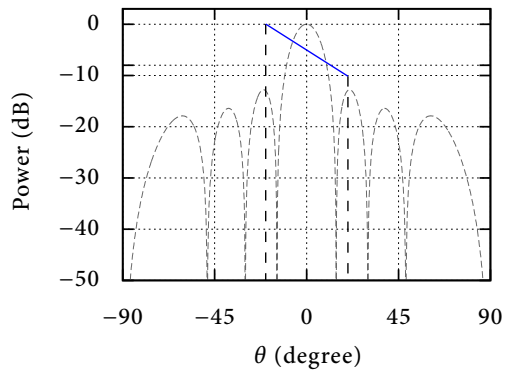

FigURE 2.13 - Desired asymmetry

Note-To circumvent many of the aforementioned problems, a form of assisted DoA estimation could be used [TB:2]. This defers the responsibility of localization and tracking to another (matured) technology (e.g., [93]). Even the mobile device itself could broadcast its position based on GSM [125] or indoor GPS [16]. However, this route has not been explored due to elegance and deployment considerations.

\section{Approach}

To the best of the author's knowledge, there is no technology independent adaptive beamforming method that operates solely on the combined output $y[k]$. With this constraint, the options for tracking are essentially reduced to scanning, which is used mostly for radar applications and is not particularly efficient in communications. Viz., a cluster of scan angles would need to be evaluated periodically to make sure that the one yielding the best output is in use [67]. Even when this cluster comprises only three scan angles (i.e., the minimum to cover the close vicinity of the peer device's last know position), there will still be two patterns to evaluate, that do not directly contribute to a good connection. For a planar array this cluster will obviously be much more extensive. Furthermore, when a sweep is performed it may be disruptive for communications. If the beam is not properly aligned with the DoA, the resulting loss in signal strength leads to a higher BER, or even complete connection loss when the DoA coincides with one of the pattern's nulls.

Since the beampattern describes a known relation between the elevation angle $\theta$ and the output $y[k]$, the power level can be monitored to serve as an indication for the correct steering direction. However, the symmetric shape of a conventional beampattern leaves interpretation of power ambiguous, as illustrated in figure 2.12. In this example, when the power measures $-8 \mathrm{~dB}$ with respect to the (normalized) $0 \mathrm{~dB}$ level of the MRA, it could mean a DoA of either $19^{\circ}$ or $42^{\circ}$. Consequently, both the $20^{\circ}$ steered pattern and $40^{\circ}$ pattern still need to be evaluated. By giving the beampattern a shape in which the relation between angle and power is unique, such as the impression given by figure 2.13 , this inefficiency could be eliminated. In the remainder of the thesis, that idea is investigated in more detail. 


\subsection{Conclusions}

A mathematical background for antenna arrays was given, which is sufficiently generic and rigorous for the following chapters. Since most of the annotations are based on [123] and [43], these references could be consulted for additional details.

The compatibility of optical (all-analog) beamforming and adaptive array algorithms has also been examined. It can be concluded that the lack of individually sampled channels, which is inherently associated with analog beamforming, is a serious impediment for most of these algorithms. Literature does report a handful of techniques based on applying perturbation sequences to the excitation control voltages. However, this requires continuously tunable phase differences while chapter 1 explained that in a high bit-rate mm-wave communication system, switched optical delay lines are preferred [28]. That means localization and tracking will be limited to scan-based algorithms. Scanning exhibits some inefficiencies in the context of a communication system. Most notably that frequent misalignment of the main beam can be disruptive for the communication. To alleviate these problems, it is proposed that the power level at the combined output of the beamformer is monitored while the shape of the beampattern is designed such that it features a unique power-to-angle relation. The next chapter will start by showing how such a shaped beampattern is obtained. 


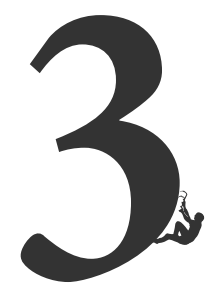

\title{
LINEAR ARRAYS:
}

\section{ANALYSIS AND SYNTHESIS}

\begin{abstract}
Aвstract - The principal objective in this chapter is to explain how one exercises control over the shape of the (power) pattern produced by a linear antenna array. Only the array's excitations will be of interest hereby. The relative positions of the antenna elements remain fixed. Specifying a desired shape, and approximate it by manipulating the array excitations, is known as shaped-pattern synthesis. In particular, this chapter will focus on shapedpattern synthesis for uniformly spaced linear arrays. By assuming that the array elements are equispaced, it is possible to employ analytical techniques. This allows for very precise control over the power pattern, which is a highly desirable feature if it is to be shaped for tracking purposes.
\end{abstract}

HE act of synthesis starts by specifying a desired pattern. When the procedures presented in this chapter are followed, this is typically done by distinguishing a shaped (null-free) region and a sidelobe region. The desired height of each sidelobe is specified individually, while the null-free region is specified using a shape function $S(\theta)$ on the interval $\left[\theta_{0}, \theta_{1}\right]$. This shape is approximated by removing nulls that intersperse the (side)lobes between $\theta_{0}$ and $\theta_{1}$. Essentially this merges several lobes into one beam that oscillates around $S(\theta)$. It is in the interest of tracking to keep the ripple that is created by this behavior as small as possible. To reach this goal, the positions of the array elements are assumed to be fixed along the $x$-axis, with a common spacing between them (\$2.1.1). It is argued here that many practical applications will experience no problems by assuming this property. For the same reason, it shall be assumed that all the elements in the array have a congruent orientation. When those conditions hold, it is possible to reason about the response of the array in an analytical sense. A (quasi-)analytical synthesis method ${ }^{1}$ can also be devised, which is capable of controlling the ripple of the pattern with extreme precision $(\leq 0.1 \mathrm{~dB})$.

${ }^{1}$ Quasi to indicate that the procedure is iterative. 
The array factor will first be analyzed more thoroughly. After a series of mathematical manipulations, the expression can be formulated as a standard polynomial. This so called Schelkunoff transformation links the array factor to the study of polynomials, so a large body of mathematical techniques becomes available to control the shape of the pattern. A synthesis method due to R.S. Elliott and H.J. Orchard [91], which relies heavily on the Schelkunoff transformation, is used to synthesize the shaped patterns needed for tracking. To demonstrate this method's capabilities and limitations, the pattern of a 16-element $1 / 2 \lambda$ spaced array is shaped for tracking purposes. The last section in this chapter also mentiones several alternative (numerical) synthesis algorithms. Although those are more generic and could lift the assumptions on the position and orientation of the array elements, it will be shown that an analytical solution leads to a ripple that is more suitable for DoA estimation. Finally, it is worthwhile to mention that the techniques developed in this chapter are also the foundation of pattern synthesis for regularly structured planar arrays (chapter 6).

\subsection{ANALYSis}

Recall from the previous chapter that the far-field array factor of an equispaced $N$-element linear antenna array is evaluated by

$$
F(\theta)=\sum_{n=0}^{N-1} I_{n} e^{j n k d \sin (\theta)}, \quad-90^{\circ} \leq \theta \leq 90^{\circ}
$$

with $I_{n}$ the (complex) excitation of the $n^{\text {th }}$ antenna element, $k$ the frequency wavenumber and $d$ the inter-element spacing. The elevation angle $\theta$ is measured in degrees from zenith, whereas some textbooks (e.g., [123]) prefer to work in radians. Although this does not change the expressions used in this chapter, one should be aware of the units when evaluating trigonometric functions. Power $|F(\theta)|^{2}$ is usually the most interesting aspect of the array factor. Plotting the power normalized on a logarithmic scale gives the well known beampattern, shown again in figure 3.1 for a $1 / 2 \lambda$-spaced 8 -element array. Formally figure 3.1 should be referred to as the power pattern [43]. However, power- and beampattern are used interchangeably here. Eq. 3.1 itself will be referred to as the array factor in $\theta$-space ( $F_{\theta}$ in short).

Mathematically it is convenient to apply the substitution

$$
\psi=k d \sin (\theta)=\frac{2 \pi d}{\lambda} \sin (\theta)
$$

where $\lambda$ is the wavelength of the center frequency, so that the array factor can be written as

$$
F(\psi)=\sum_{n=0}^{N-1} I_{n} e^{j n \psi}, \quad-\pi \leq \psi \leq \pi
$$




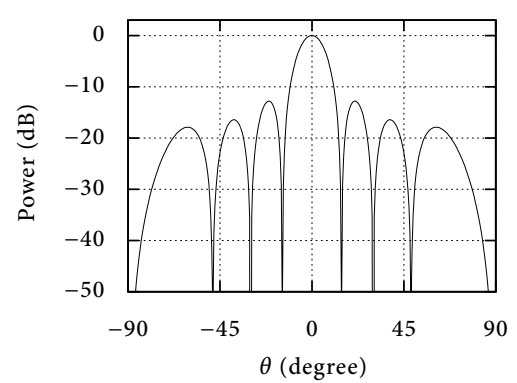

FIGURE 3.1 - Power pattern in $\theta$-space.



FIGURE 3.2 - Power pattern in $\psi$-space.

This form will be referred to as the array factor in $\psi$-space $\left(F_{\psi}\right)$. Note that $\psi$ is dimensionless, not in radians as its range may suggest. Figure 3.2 depicts again the power of an eight element array, but now in $\psi$-space. For this figure is follows from $d=1 / 2 \lambda$ that

$$
\psi=\pi \sin (\theta)
$$

and subsequently that the visible region in $\psi$-space is $-\pi \ldots \pi$. More generally, the visible region of $F(\psi)$ can be expressed as

$$
\bar{\psi}=2 d \frac{2 \pi}{\lambda}
$$

centered around $\psi=0$. It should be evident from eq. (3.2) and (3.5) that the $\psi$ substitution is limited to arrays with uniformly spaced elements. Because many of the principles discussed next are based on $F_{\psi}$, this property is assumed to hold unless specified otherwise.

Although there are substantial differences between figure 3.1 and figure 3.2, the relation between $\theta$ - and $\psi$-space is defined exact, and switching from one definition to the other is straightforward. Let $d$ be expressed in terms of $\lambda$, then it holds for a certain angle $-90^{\circ} \leq \theta \leq 90^{\circ}$ that

$$
\left|F_{\theta}(\theta)\right|=\left|F_{\psi}(2 \pi d \sin (\theta))\right|=\left|F_{\psi}(\psi)\right|
$$

and vice versa for $-\pi \leq \psi \leq \pi$ that

$$
\left|F_{\psi}(\psi)\right|=\mid F_{\theta}\left(\sin ^{-1}\left(\frac{\psi}{2 \pi d}\right)|=| F_{\theta}(\theta) \mid\right.
$$

The $\psi$-space beampattern can thus replace its $\theta$ counterpart if needed. Moreover, $\psi$-space is the antenna array equivalent of spectral analysis for time domain signals. 


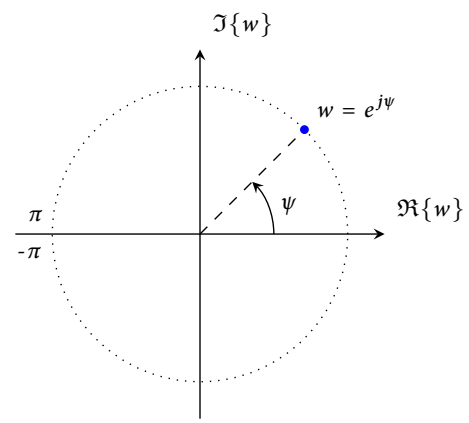

FIGURE 3.3 - Relation between $\psi$ and $w$

This mathematical duality enables one to apply various weighting functions, that have also found widespread use in for example FIR filter design, to the excitations of an equispaced linear antenna array [123]. Typical weightings such as the Hamming and Kaiser window give limited control over sidelobes and beamwidth. A shaped beampattern cannot be obtained this way. For shaped patterns, the $\psi$-space only serves as an intermediate step.

\subsubsection{The SChELKUnOFF TRANSFormation}

The next logical substitution for eq. 3.3 is

$$
w=e^{j \psi}
$$

so that the array factor is represented by the polynomial

$$
F(w)=\sum_{n=0}^{N-1} I_{n} w^{n}
$$

Note that $w$ lies in the complex plane ${ }^{2}$, as shown in figure 3.3. Since $\psi$ is the argument of $w$, and therefore always real, $w$ is constrained to the circumference of a unit circle. This also means that (given a uniform spacing of $d=1 / 2 \lambda$ ) there is a one-to-one relationship between $\psi$ and $w$. Schelkunoff [107] was the first to realize this. The unit circle of figure 3.4 is therefore often referred to as the Schelkunoff unit circle and eq. 3.9 as the Schelkunoff unit circle representation. By the fundamental theorem of algebra, eq. 3.9 can be factored into $(N-1)$ binomials. Accordingly,

$$
F(w)=I_{N-1} \prod_{n=1}^{N-1}\left(w-w_{n}\right)
$$

\footnotetext{
${ }^{2}$ This transformation is (but a minus sign in the exponent) analogous to the $z$-transform.
} 


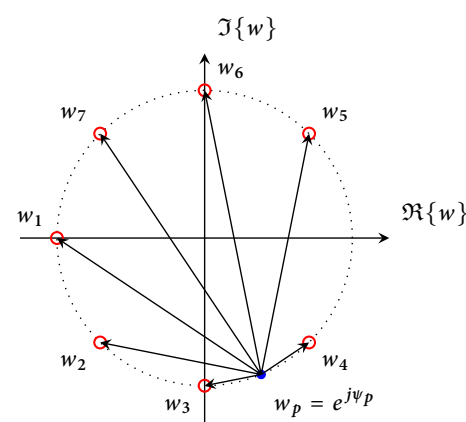

FIGURE 3.4 - Root positions

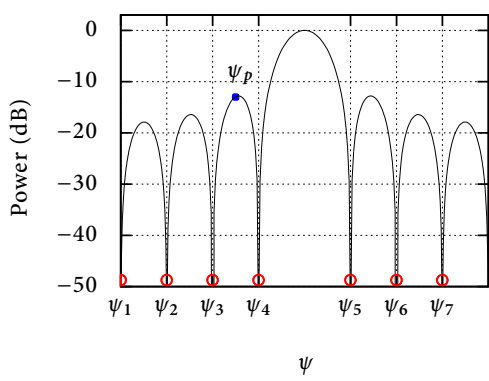

FIGURE 3.5 - Null positions

can be used for the $\psi$-space array factor (and as a surrogate for the beampattern), where $w_{n}=e^{j \psi_{n}}$ are the roots of the polynomial [107]. Ordering of the $w_{n}$ is in principle irrelevant for the beampattern. However, the convention adopted here is to index the roots in eq. 3.10 in order of occurrence, starting from $\psi=-\pi$ going counter clockwise to $\psi=\pi$ (figure 3.4 ).

Analogously with the geometric evaluation of transfer functions by pole zero plots [111], the beampattern can now also be seen as the product of distances between $w$ and the roots $w_{n}$ [86]. Figure 3.4 illustrates this concept for a given position $w_{p}=e^{j \psi_{p}}$. Realizing this, it is evident that $F(w)$ will be zero when $w$ coincides with one of the roots $w_{n}$, as it entails a multiplication by zero. A convenient relation between the roots in eq. 3.10 and the nulls in $F(w)$ follows, as seen in figure 3.5. The peaks in the beampattern also roughly correspond (but not exactly) to the center between two roots (as illustrated by $\psi_{p}$ between $w_{3}$ and $w_{4}$ in figure 3.4).

Eq. 3.10 may provide additional insight to the concept of visible region (\$2.1.4). For arrays with an element separation of exactly $1 / 2 \lambda$, the range of $\psi$ according to eq. 3.5 is $2 \pi$. This corresponds exactly to one cycle around the unit circle for $w$, as $\theta$ varies from $-90^{\circ}$ to $90^{\circ}$. In this case there is a one-to-one relation between the circumference of the unit circle and the physical range of the antenna array (i.e., $\theta=-90^{\circ} \ldots 90^{\circ}$ ). If $d$ is less than $1 / 2 \lambda$, the range of $w$ is less than $2 \pi$ and as such, only a part of the unit circle is described. On the other hand, when $d$ is larger than $1 / 2 \lambda$ the path followed by $w$ will overlap itself because $\bar{\psi}>2 \pi$. Figure 3.6 depicts this for uniform spacings of $\lambda$ and $1 / 4 \lambda$. The corresponding beampatterns can be found in figure 3.7. Since the physical range of $\theta$ does not change, the pattern for $d=1 / 4 \lambda$ will only feature four nulls given that $w$ only encounters four out of seven roots. Effectively this can be seen as broadening of the beampattern. The other way around, the pattern of a $\lambda$ spaced array will exhibit spatial aliases because the roots are encountered more than once.

Following all the above relations, changing to the position of the roots in the complex $w$-plane can be coupled to shaping of the beampattern in $\theta$-space. To exem- 


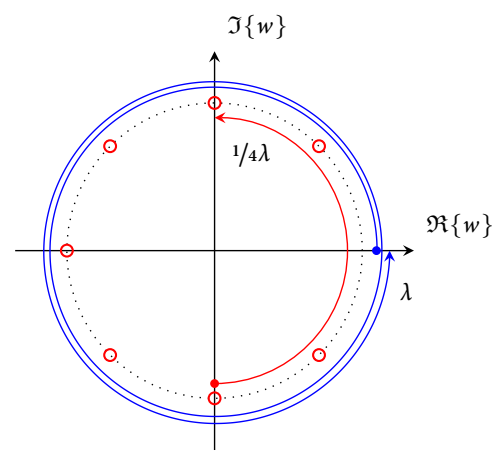

FIgURE 3.6 - Visible region in $w$-space

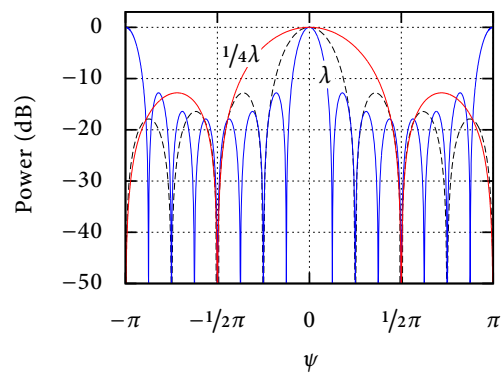

FIGURE 3.7 - Visible region in $\psi$-space

plify this, the fifth root from figure 3.4 (i.e., $w_{5}$ ) has been rotated $\delta \psi=1 / 8 \pi \mathrm{rad}$ (counterclockwise) in figure 3.8. Figure 3.9 reveals two interesting changes in the beampattern that emanates from this adjustment. Firstly, the lobe between $w_{5}^{\prime}$ and $w_{6}$ is narrower than the original. Secondly, the corresponding peak value has been lowered. On the other side, the rotation increases the distance between $w_{4}$ and $w_{5}^{\prime}$, which has lead to a wider main beam with a peak value that is higher compared to the sidelobes. From this it can be concluded that, besides placing nulls at specified locations, repositioning the roots on the unit circle allows one to change the position and height of the lobes in the beampattern.

\section{Collective root rotation}

By rotating roots collectively, over the same angular distance, their relative positions do not change. A common displacement therefore does not change the shape of the beampattern. It does change the absolute positions of the peaks and nulls. This property is interesting as it enables the pattern to move freely in $\psi$-space (i.e., steer it), without affecting the shape. Figure 3.10 and figure 3.11 illustrate this for the

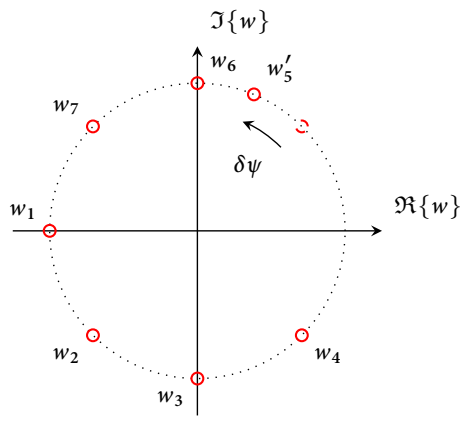

FIGURE 3.8 - Angular root displacement

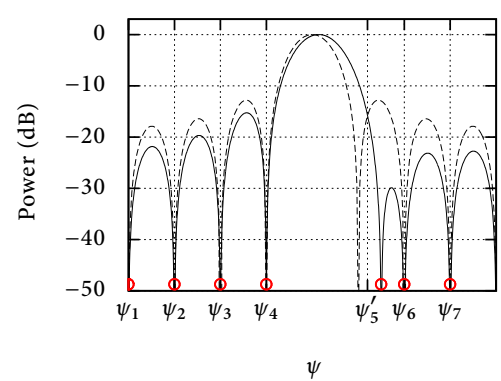

FIGURE 3.9 - Altered beampattern 




FIgURE 3.10 - Common root rotation

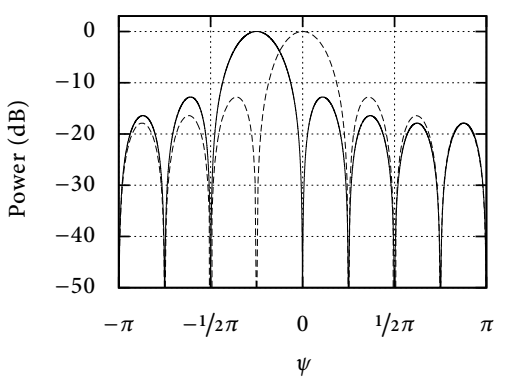

FIgURE 3.11 - Shifted beampattern

common rotation $\delta \psi=-1 / 4 \pi$, causing the entire pattern to shift $1 / 4 \pi$ rad to the left.

Finding array excitations from roots

Before a synthesis method is developed based on these findings, it is useful to know how the complex array excitations $I_{n}$ can be found from the corresponding roots. This requires that the factors of eq. 3.10 are expanded. Consider for example an equispaced array with $N=3$ elements. For simplicity, let $I_{N-1}$ be 1 such that

$$
F(w)=\prod_{n=1}^{N-1}\left(w-w_{n}\right)
$$

with $w_{1}=z_{0}$ and $w_{2}=z_{1}$ to designate the $N-1$ roots. After multiplying the factors and sorting them by order, one obtains

$$
F(w)=w^{2}-w\left(z_{0}+z_{1}\right)+z_{0} z_{1}
$$

for which it holds that

$$
w^{2}-w\left(z_{0}+z_{1}\right)+z_{0} z_{1}=\sum_{n=0}^{2} I_{n} w^{n}=I_{1}+I_{2} w+I_{3} w^{2}
$$

From the right hand side of eq. 3.13, the excitations $I_{n}$ can be derived

$$
\begin{aligned}
& I_{1}=z_{0} z_{1} \\
& I_{2}=-\left(z_{0}+z_{1}\right) \\
& I_{3}=1
\end{aligned}
$$




\subsection{SyNTHESIS BASED ON POLYNOMIALS}

Elliott was among of the first to develop a systematic procedure to synthesize beampatterns based on Schelkunoff's formulations [40]. This method can be used to specify the height of each sidelobe individually. Although synthesis of null-free shaped beams is not possible, the method introduced various concepts that are fundamental to the more advanced algorithms that can $[44,91]$. This section starts by explaining these fundamentals. Improvements will then gradually be introduced in the subsequent sections, which eventually lead to the Orchard-Elliott synthesis procedure mentioned in the introduction.

Elliott started by writing eq. 3.10 as

$$
F(w)=C \frac{\prod_{i=1}^{N-1}\left(w-w_{n}\right)}{w^{N / 2}}
$$

with $I_{N-1}=1$. The term $1 / w^{N / 2}$ is added to make the phase of the array factor $(\angle F)$ independent of $w$. Although not relevant for the shape of the power pattern, this representation will be used for consistency with [40]. The constant $C$ is a complex value whose amplitude is used to normalize the level of the beampattern (usually to $0 \mathrm{~dB}$ ) and keep the phase of $F$ constant. If the phase of $F(w)$ is $\varphi$, then the argument of $C$ should be chosen $-\varphi$ such that eq. 3.14 is a pure real function of which the sign changes from one lobe to the next.

A starting pattern $F_{0}$ needs to be chosen as an approximation to the desired pattern $F$. This starting pattern can be expressed as

$$
F_{0}(w)=C_{0} \frac{\prod_{n=1}^{N-1}\left(w-\stackrel{\circ}{*}_{n}\right)}{w^{N / 2}}
$$

where $C_{0}$, and $\stackrel{\circ}{w}_{n}$ are known initial values. Assuming that the roots of $F(w)$ are close to $F_{0}(w)$, linear perturbations $\delta \psi_{n}$ (i.e., small angular displacements) are applied to the root positions of the initial pattern

$$
\psi_{n}=\stackrel{\circ}{\psi}_{n}+\delta \psi_{n}
$$

When $\delta \psi_{n}$ is sufficiently small, it holds that

$$
w_{n}={\stackrel{\circ}{w_{n}}}^{j \delta \psi_{n}} \simeq{\stackrel{\circ}{w_{n}}}_{n}\left(1+j \delta \psi_{n}\right)
$$

and $F(w)$ can thus be written as 


$$
\begin{aligned}
F(w) & =\left(C_{0}+\delta C\right) \frac{\prod_{n=1}^{N-1}\left(w-\stackrel{\circ}{w}_{n} e^{j \delta \psi_{n}}\right)}{w^{N / 2}} \\
\simeq\left(C_{0}+\delta C\right) & \frac{\prod_{n=1}^{N-1}\left[w-\stackrel{\circ}{w}_{n}\left(1+j \delta \psi_{n}\right)\right]}{w^{N / 2}}
\end{aligned}
$$

The additional perturbation $\delta C$ allows adjustment of the power level, so that the height of the lobes in $F_{0}(w)$ and $F(w)$ can be compared. It also relates $\varphi_{0}$ and $\varphi=\varphi_{0}+\delta \varphi$ such that both $F_{0}(w)$ and $F(w)$ are pure real functions, which would be relevant if one is interested in the phase of the pattern. By retaining only the zeroth and first order terms from the Taylor expansion of eq. 3.18, the result of perturbing the root position can be expressed as [40]

$$
\frac{F(w)-F_{0}(w)}{F_{0}(w)}=\frac{\delta C}{C_{0}}-j \sum_{n=1}^{N-1} \frac{\stackrel{\circ}{w}_{n}}{w-\stackrel{\circ}{n}_{n}} \delta \psi_{n}
$$

Let $w_{p}$ denote the position on the Schelkunoff unit circle that corresponds to the maximum of the $p^{\text {th }}$ lobe in $F_{0}(w)$. There are $N-1$ lobes and thus $N-1$ distinct values for $w_{p}(p=1,2, \ldots, N-1)$. Inserting these in eq. 3.19 yields a system of $N-1$ linear equations with $N$ unknowns: $\delta C$ and $\delta \psi_{1}, \ldots, \delta \psi_{N-1}$. This system is not deterministic (i.e., not square), which can be traced back to the fact that the entire set of roots can be rotated around the unit circle freely, without affecting their relative positions. To prevent this from happening, one of the root positions needs to be fixed. It is convenient to anchor the position of the first or last $(N-1)^{\text {th }}$ root at $\psi=\pi[40]$. This results in a set of equations

$$
\frac{F\left(w_{p}\right)-F_{0}\left(w_{p}\right)}{F_{0}\left(w_{p}\right)}=\frac{\delta C}{C_{0}}-j \sum_{n=1}^{\mathrm{N}-2} \frac{\stackrel{\circ}{w}_{n}}{w_{p}-\stackrel{\circ}{w}_{n}} \delta \psi_{n}
$$

for which a unique solution can be found. In summary, the known quantities in eq. 3.20 are:

$$
\begin{aligned}
F_{0}\left(w_{p}\right) & \text { the height of the } p^{\text {th }} \text { lobe in the initial pattern; } \\
F\left(w_{p}\right) & \text { the corresponding lobe height in the desired pattern; } \\
C_{0} & \text { the normalization factor for the initial pattern; } \\
w_{p} & \text { the location of the } p^{\text {th }} \text { lobe in the initial pattern; } \\
\dot{w}_{n} & \text { the root location of } n^{\text {th }} \text { root in the initial pattern. }
\end{aligned}
$$

Solving the system, by for example row reduction or matrix inversion, will govern $\delta C$ and $\delta \psi_{1}, \ldots, \delta \psi_{N-1}$. From these values, the new $C$ and $w_{n}$ can be found for use in eq. 3.14. If the result does not approximate the desired pattern close enough, the procedure can be repeated until it does. Convergence is rapid enough to allow practically any pattern as the starting point. 


\subsubsection{EXAMPLE: ANGULAR ROOT DISPLACEMENT SYNTHESIS}

For demonstration purposes, the pattern of a 16 -element $1 / 2 \lambda$-spaced linear array shall be synthesized to feature the 'ramp-like' shape that was shown in figure 2.13. This shape is specified for the range $\left[0^{\circ}, 40^{\circ}\right]$ to facilitate a mathematical convenient shape definition $S_{r}(\theta)$. Since a truly shaped region (free of nulls) is not yet possible, the height of each lobe between $\theta_{0}=0^{\circ}$ and $\theta_{1}=40^{\circ}$ is required to end up on the contour of $\mathrm{a}-10 \mathrm{~dB}$ per $40^{\circ}$ ramp shape. In decibels, this contour is defined by

$$
S_{r}(\theta)=-\frac{1}{4} \theta, \quad 0^{\circ} \leq \theta \leq 40^{\circ}
$$

The height of the remaining lobes will be uniformly suppressed to $-25 \mathrm{~dB}$. In total eight iterations are performed. From a selection of those iterations, supplementary numerical data is provided in table A.1, found in appendix A.

Uniform excitations $\left(\forall n \mid I_{n}=1\right)$ are the staring point for this example. The corresponding pattern features nulls at $\dot{\leftrightarrow}_{n}= \pm 1 / 8 \pi, \pm 2 / 8 \pi, \ldots, \pm 7 / 8 \pi$ and $\pi$, from which the initial $\stackrel{\circ}{w}_{n}$ can immediately be determined by filling in eq. 3.8

$$
{\stackrel{\circ}{\mathcal{W}_{n}}}_{n}=e^{j \stackrel{\circ}{\psi}_{n}}
$$

The peak of the power pattern is normalized to $1(0 \mathrm{~dB})$. For an $N$ element array, this means that the amplitude of the complex normalization factor $C_{0}$ should be $1 / N$. The phase of the array factor with uniform excitation is $\varphi=0$, which leads to

$$
C_{0}=\frac{1}{N} e^{-\varphi}=\frac{1}{16}
$$

With the initial values known, the next step is to find the maxima of the current beampattern. Both location $\psi_{p}$ and corresponding peak value $\left|F_{0}\left(w_{p}\right)\right|$ need to be found for the $p^{\text {th }}$ lobe. These could be determined by finding the intersection of the first derivative of $\left|F_{0}\right|$ with 0 . The desired pattern for eq. 3.20 is based on eq. 3.21. However, since this is a definition in terms of $\theta$, it is convenient to define a $\psi$-space equivalent ramp shape

$$
S(\psi)=\frac{-10}{\pi \sin \left(40^{\circ}\right)} \psi, \quad 0 \leq \psi \leq \pi \sin \left(40^{\circ}\right)
$$

which can be evaluated at the locations of the peaks $\left(\psi_{p}\right)$. The perturbations $\delta \psi_{n}$ are found by inserting the known values in eq. 3.20, and solving the set of equations. The updated roots

$$
w_{n}=\stackrel{\circ}{w}_{n} e^{\left(\delta \psi_{n} j\right)}
$$


and normalization constant

$$
C=C_{0}+\delta C
$$

give a pattern which is closer to $S$ with each iteration performed. After the eighth iteration, the roots have settled at the locations shown in figure 3.12. Plotting the power pattern in $\theta$-space reveals that the height of the designated lobes follow the ramp shaped contour as intended. The major shortcoming of this pattern is obviously the presence of deep nulls in the shaped region.

\subsubsection{NULL-FREE SHAPED REGIONS}

Confining the roots to the unit circle is not a requisite for physically realizable patterns. In fact, moving selected roots radially off the Schelkunoff unit circle fills the corresponding nulls in the beampattern. Suppose that, as depicted in figure 3.14, root $w_{5}$ from the $\$ 3.1$ example is moved off the unit circle by a distance $b_{m}$. It can be observed in figure 3.15 that the corresponding null in the pattern disappears. This suggests a way to systematically fill all the nulls within a certain range. Based on this principle, Elliott presented an extension in [44] to address the nulls in the shaped region. In this work, two angular regions are distinguished: region $I$, where the procedure 'fills' $N_{1}$ nulls up to the level specified by the shaping function $S$; and region $I I$ in which nulls remain unaltered.

One starts again with a polynomial representation of the array factor

$$
F_{1}(w)=C_{1} \prod_{n=1}^{N-1}\left(w-w_{n, 1}\right)
$$

where the added subscript (1) indicates pre radial root displacement but post angular root displacement (i.e., patterns shaped as explained in the previous section). If $\left|F_{1}(w)\right|$ is interpreted as the product of the distances from $w$ to all roots $w_{n, 1}$, it

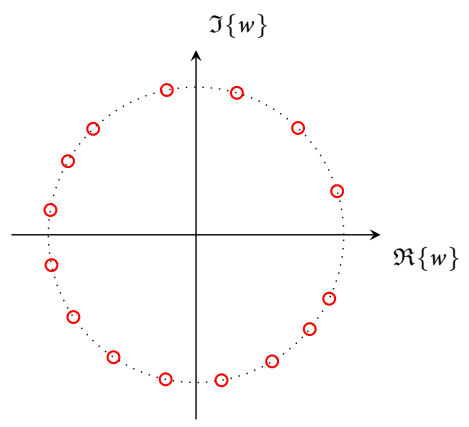

FIGURE 3.12 - Root locations

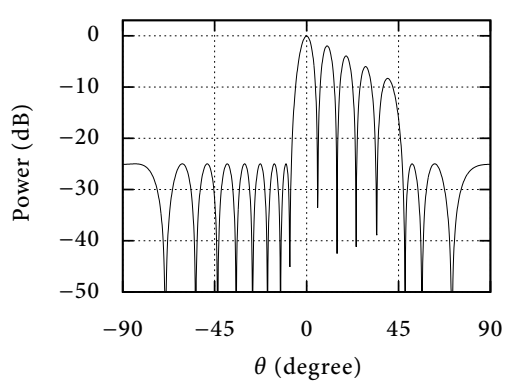

FIGURE 3.13 - 'Ramp-shaped' pattern 
follows that $\left|F_{1}\left(w_{n, 1}\right)\right|=0$ when $w$ coincides with $w_{n, 1}$. By moving a particular root $w_{m, 1}$ to $\left(1+b_{m}\right) w_{m, 1}$, where $b_{m} \neq 0$, it will never be encountered by $w$ since that path is confined to the circumference of the unit circle. The value of $\left|F_{1}\right|$ at $w=w_{m, 1}$ is then given by

$$
\left|F_{1}\left(w_{m, 1}\right)\right|=\left|C_{1} b_{m} \prod_{n=1}^{N-2}\left(w_{m, 1}-w_{n, 1}\right)\right|
$$

Note that herein the $m^{\text {th }}$ factor was taken outside product in comparison to eq. 3.27. A desired amount of filling (i.e., the left side of the equation) can be determined from the prescribed shape $S$. Since $C_{1}$ is given and the product of factors in eq. 3.28 can also be computed, a solution for $b_{m}$ can be found. By repeating this for all $q=N_{1}$ roots in region $\mathrm{I}$, a new response can be defined as

$$
F_{2}(w)=C_{2} \prod_{n=1}^{q}\left(w-w_{n, 2}\right) \prod_{n=q+1}^{N-1}\left(w-w_{n, 1}\right)
$$

in which $w_{n, 2}=\left(1+b_{m}\right) e^{j \psi_{n, 1}}$ and $C_{2}$ a new normalization constant. The targeted nulls will be filled up to the level specified by $\left|F_{1}\left(w_{m, 1}\right)\right|=S$ in the pattern of eq. 3.29. However, this pattern will still suffer from two deficiencies. Firstly the oscillations of $F_{2}$, created by filling the nulls in region I, deviate from $S$ significantly and in an unbalanced fashion. Secondly the sidelobes will be slightly lower than originally specified. Although a lower sidelobe level is not necessarily problematic, it is relatively easy to correct. One determines the difference between the desired and actual sidelobe level after having filled the nulls. These differences are then taken into account when synthesizing the starting pattern $F_{1}$, so that the sidelobes in $F_{2}$ will arrive at the specified height. Improving region $I$ is more difficult and involves trial and error adjustment to the $b_{m}$ values [44].

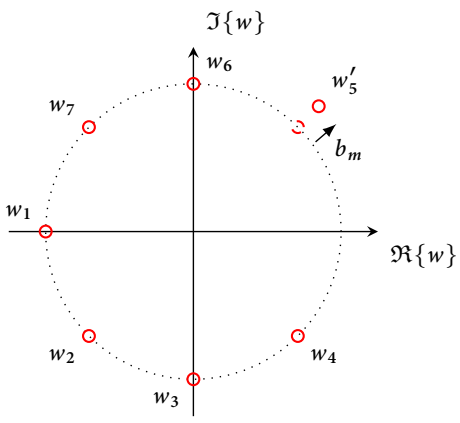

FIGURE 3.14 - Radial displacement $b_{m}$

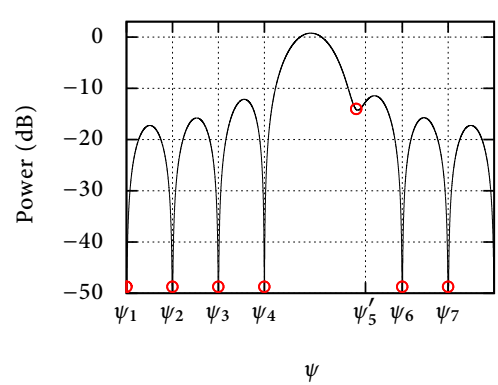

FIGURE 3.15 - Null filling in pattern 


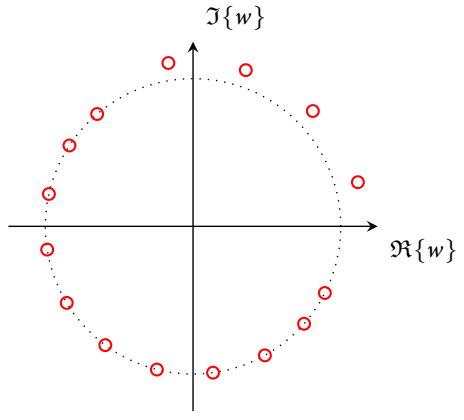

FIgURE 3.16 - Off-circle roots

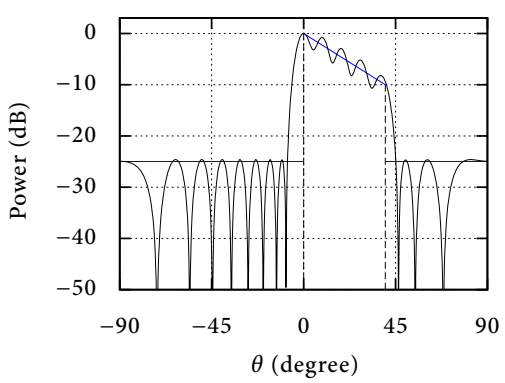

FIgURE 3.17 - Null free in region I

\subsubsection{EXAMPLE: RADIAL ROOT DISPLACEMENT SYNTHESIS}

The pattern from $\$ 3.2 .1$ is used to illustrate the null filling procedure, which starts by identifying the roots that have to be displaced radially. By the indexing convention defined in $\$_{3.1 .1}, w_{9}, w_{10}, w_{11}$ and $w_{12}$ reside in region I. A fill level corresponding to the positions $\psi_{9}, \psi_{10}, \psi_{11}$ and $\psi_{12}$ is determined from $S(\psi)$ (eq. 3.24). Expressed in decibels, these levels are listed in the second column of table 3.1. Their nonlogarithmic counterparts, found in the third column, are used as the left side of eq. 3.28. When the factors in eq. 3.28 have also been calculated (i.e., the fourth column of table 3.1), sufficient information is available to find the required radial displacements $b_{m}$ (fifth column). Having applied these values to eq. 3.29, the sidelobes in region II are found to be roughly $1.4 \mathrm{~dB}$ below the specified $-25 \mathrm{~dB}$. The pattern from example 3.2.1 was therefore resynthesized with a sidelobe level of $-23.6 \mathrm{~dB}$ to compensate. Finally, as per suggestion of [44], some minor adjustment were made in $b_{m}$ to improve $\left|F_{2}\right|$ 's oscillatory behavior with respect to $S$. The new values are: $b_{1}^{\prime}=0.85 b_{1}, b_{2}^{\prime}=0.8 b_{2}, b_{3}^{\prime}=0.8 b_{3}$ and $b_{4}^{\prime}=0.85 b_{4}$. The result can be observed in figures 3.16 and 3.17 .

The oscillating behavior of $|F(\theta)|$ around $S(\theta)$ is called the ripple of the shaped region. For tracking purposes, this characteristic is very important. To a large extent it determines how accurately the DoA can be derived from the beampattern's shape. Pursuing patterns with a low ripple is therefore one of the major objectives

TABLE 3.1 - Root displacement data for null filling

\begin{tabular}{lcccc}
$w_{n}$ & $S\left(w_{n}\right)(\mathrm{dB})$ & $S\left(w_{n}\right)$ & $\prod$ & $b_{m}$ \\
\hline$w_{9}$ & -0.478 & 0.947 & 5.180 & 0.183 \\
$w_{10}$ & -2.900 & 0.716 & 4.554 & 0.157 \\
$w_{11}$ & -5.363 & 0.539 & 3.709 & 0.145 \\
$w_{12}$ & -8.100 & 0.394 & 2.824 & 0.139
\end{tabular}


in this thesis. The metric used to quantify the ripple, e.g. $0.5 \mathrm{~dB}$, is defined as $\max \{F-S\}+|\min \{F-S\}|$. This is shown in figure 3.18.

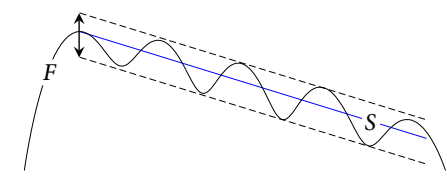

FIGURE 3.18 - Ripple size

Although fairly balanced, the ripple seen figure 3.17 is in excess of $4 \mathrm{~dB}$ and highly impractical for tracking. Moreover, the trial-and-error type of balancing that was employed to obtain this pattern is quite cumbersome. The following section presents Orchard-Elliott synthesis. Herein, the final improvement is a means to systematically reduce and balance the ripple during synthesis.

\subsection{OrChaRd-ELLIOTt SHAPED-PATTERN SYNTHESIS}

The key to having better control over the ripple in the shaped region of a beampattern is to apply both radial and angular perturbations to the roots in region I simultaneously. In 1985, Orchard, Elliott and Stern presented this idea in [91]. To date this method is still relevant for its unparalleled control over the ripple in the null-free region.

As before, the algorithm uses the polynomial representation of the array factor

$$
F(\psi)=\sum_{n=0}^{N-1} I_{n} e^{j n \psi}=I_{N} \prod_{n=1}^{N-1}\left(w-w_{n}\right)
$$

with the usual substitutions $w=e^{j \psi}$ and $\psi=2 \pi d \sin (\theta)$. By decomposing $w_{n}$ into $e^{a_{n}+j b_{n}}$, i.e. a radial $\left(a_{n}\right)$ and an angular $\left(b_{n}\right)$ position, the power pattern becomes

$$
|F(\psi)|^{2}=\left|I_{N}\right|^{2} \prod_{n=1}^{N-1}\left[1-2 e^{a_{n}} \cos \left(\psi-b_{n}\right)+e^{2 a_{n}}\right]
$$

The angular position of the $(N-1)^{\text {th }}$ root will be fixed at $\psi=-\pi\left(a_{N-1}=0\right.$ and $\left.b_{N-1}=-\pi\right)$, so the pattern expressed in decibels is 


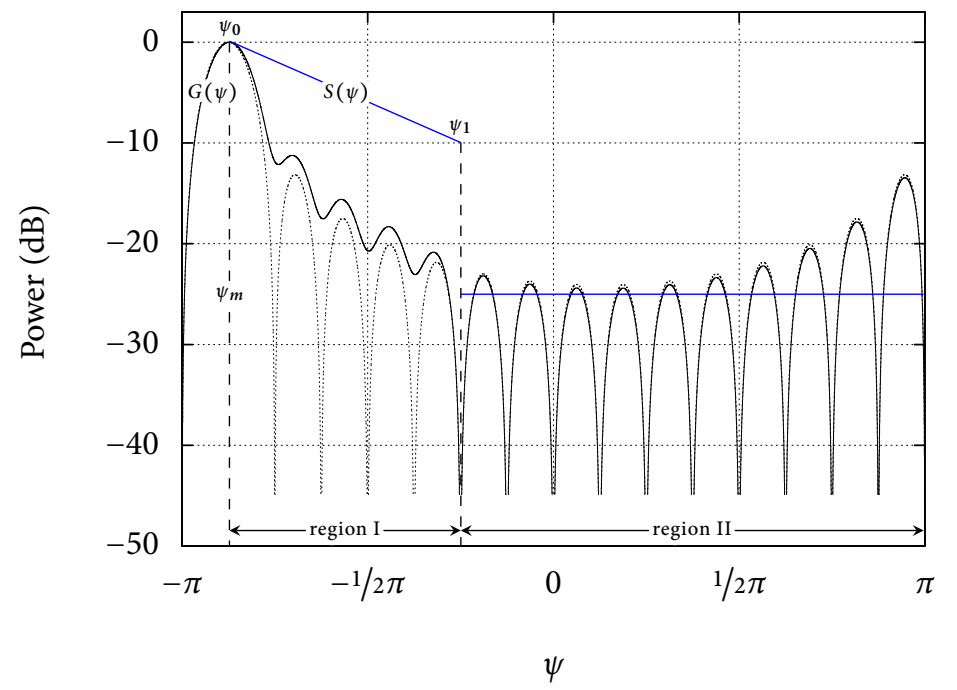

FIGURE 3.19 - Starting pattern $G \psi$, region definitions and desired shape $S(\psi)$

$$
\begin{aligned}
G(\psi) & =\sum_{n=1}^{N-2} 10 \log _{10}\left[1-2 e^{a_{n}} \cos \left(\psi-b_{n}\right)+e^{2 a_{n}}\right] \\
& +10 \log _{10}[2(1+\cos (\psi))]+C_{1}
\end{aligned}
$$

were the constant $C_{1}$ is used to normalize the peak value to a convenient reference. $G(\psi)$ is arranged such that the main lobe lies adjacent to $-\pi$, as indicated for $N=16$ by the dotted line in figure 3.19. This is essentially the pattern of an array with uniform excitations, but the roots are swiveled around the unit circle until the one located directly to the left of the main lobe is positioned at exactly $w=e^{j(-\pi)}$. The (non-fixed) initial root positions of this pattern are given by

$$
\begin{gathered}
b_{n}=\left(\frac{2 n}{N+1}-1\right) \pi, \quad n=2,3, \ldots, N-1 \\
a_{n}=0, \quad n=2,3, \ldots, N-1
\end{gathered}
$$

A root positioned on the unit circle (i.e., $a_{n}=0$ ) leads to $-\infty$ in $G(\psi)$, which is problematic for the algorithm because it needs to evaluate $G-S$ to find the local minima and maxima of the ripple. The roots in region I are therefore moved slightly off the unit circle. According to [91], $a_{n}=0.01$ is sufficient, but choosing different 
values for $a_{n}$ may result in quicker convergence depending on the desired shape $S$. The solid line in figure 3.19 is for example produced by $a_{n}=0.1$. On the other hand, it was also found that for large $N$ higher $a_{n}$ values can lead to an ill-conditioned ripple, wherein not all minima and maxima can be found. As a rule of thumb $a_{n}=0.1$ may be used for $N \leq 20$.

The objective shape $S(\psi)$ is defined relative to $\psi_{m}$ where $\max \{G(\psi)\}$ is located. Initially this corresponds to the main lobe of the pattern (figure 3.19). Since this peak location is dictated by the fixed root at $-\pi, S(\psi)$ will need to be given an offset $\delta \psi=\psi_{0}-\psi_{m}$ to allow the shaped region $\left[\psi_{0}, \psi_{1}\right]$ to be chosen arbitrarily

$$
S(\psi)=S(\psi-\delta \psi)
$$

After synthesis the pattern can be shifted back to its original definition, which is accomplished by rotating all roots simultaneously (\$3.1.1). This counter-intuitive arrangement of $G(\psi)$ and $S(\psi)$ has two advantages. Firstly, it is convenient for bookkeeping in the algorithm. Secondly, $\psi_{m}$ changes as $G(\psi)$ converges to $S(\psi)$. By placing $\psi_{m}$ adjacent to the fixed root, the range of region I will remain fairly stable during synthesis.

There will be $N_{1}$ roots in region I and $N_{2}$ roots in region II. Including the fixed root at $\psi=-\pi$, this amounts to $N_{1}+N_{2}+1$ roots in total. For the first $N_{1}$, both $a_{n}$ and $b_{n}$ will be adjusted. For the following $N_{2}$ roots, only $b_{n}$ can be altered since $a_{n}=0$ for region II. This corresponds to a pattern with a null-free (shaped) region created by $N_{1}$ roots, surrounded by a freely specifiable sidelobe topography. Together with the normalization factor $C_{1}$, the above gives $N_{3}=2 N_{1}+N_{2}+1$ parameters for the synthesis algorithm to optimize. These are assumed to be combined in a vector $\mathbf{f}$.

In practice $G\left(\psi, a_{n}, b_{n}, C\right)$, in short $G(\psi, \mathbf{f})$, will only approximate $S(\psi)$. The approximation will be optimal when $G(\psi, \mathbf{f})$ oscillates around $S(\psi)$ with the extrema of the ripple created by $G(\psi, \mathbf{f})-S(\psi)$ balanced. To obtain a measure of quality for this approximation, the $N_{2}$ desired peak values of the sidelobes in region II; and the $N_{1}-1$ desired minima and $N_{1}$ maxima of $G-S$ in region I are collected. If the ripple should not exceed e.g. $0.1 \mathrm{~dB}$, then the minima and maxima must be set to $\pm 0.05 \mathrm{~dB}$. Let these $g_{i}$ target values be assembled in a vector $\mathbf{g}$.

A measure for how well the current pattern approximates $S(\psi)$ is also needed. To this end, the angular locations $\psi_{i}$ corresponding to the $g_{i}$ values need to be found first. Let the vector $\psi$ denote the $\psi_{i}$ values that belong to the $g_{i}$ values, arranged the same order as $\mathbf{g}$. Once $\psi$ has been found, $G\left(\psi_{i}\right)$ is computed when $\psi_{i}$ resides in region II and $G\left(\psi_{i}\right)-S\left(\psi_{i}\right)$ when $\psi_{i}$ is in region I. This has been illustrated in figure 3.20. Let $\hat{\mathbf{g}}$ be the vector that contains these $\hat{g}$ values, ordered in the same way as $\psi$. In summary, the following data has now been collected: 


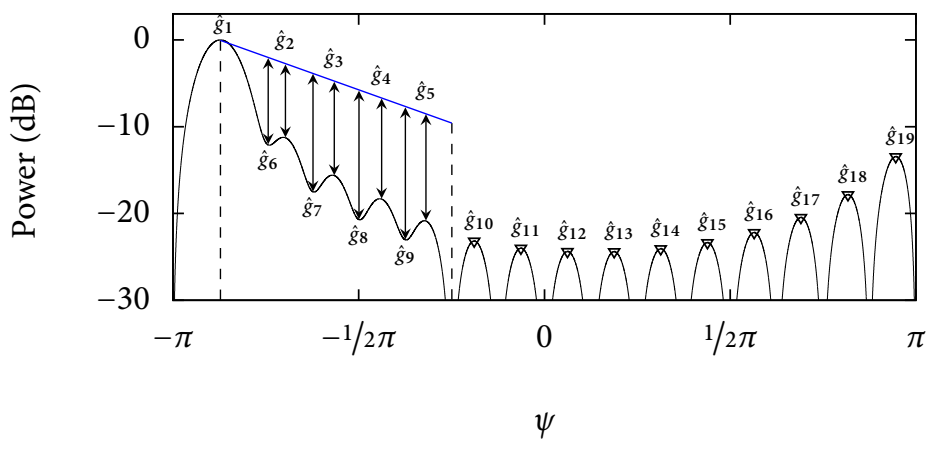

FIGURE 3.20 - Pattern performance measures. Note that the $\hat{g}$ values in region I are not the minima and maxima of the pattern itself, but originate from $G(\psi)-S(\psi)$.

f $\quad a_{n}$ (region I only), $b_{n}$ and $C$ from eq. 3.32;

g desired ripple extrema in region I and desired sidelobe heights in region II;

$\psi \quad$ angular location $\psi$ where $\psi_{i}$ corresponds to $g_{i} ;$

$\hat{\mathrm{g}} G\left(\psi_{i}, \mathbf{f}\right)$ if $\psi_{i}$ in region II and $G\left(\psi_{i}, \mathbf{f}\right)-S\left(\psi_{i}\right)$ if $\psi_{i}$ in region I.

Using the collected data, small corrections $\delta \mathbf{f}$ must be found such that $G(\mathbf{f}+\delta \mathbf{f}, \psi)$ will be a better approximation to $S(\psi)$. Applying linear perturbations $\delta b_{n}, \delta a_{n}$ and $\delta C$ to the variables of $\mathbf{f}$, Taylor expansion gives[86]

$$
\begin{aligned}
G(\mathbf{f}, \psi) & =G_{0}(\mathbf{f}, \psi)+\sum_{n=1}^{N-2} \frac{\partial G}{\partial b_{n}}\left(b_{n}-b_{n, 0}\right) \\
& +\sum_{n=1}^{N-2} \frac{\partial G}{\partial a_{n}}\left(a_{n}-a_{n, 0}\right)+\left(C-C_{0}\right)
\end{aligned}
$$

where $G_{0}$ is the starting pattern. Three different types of terms can be distinguished herein

$$
\begin{gathered}
\frac{\partial G}{\partial b_{n}}=\frac{10 \log _{10} e^{a_{n}} \sin \left(\psi-b_{n}\right)}{1-2 e^{a_{n}} \cos \left(\psi-b_{n}\right)+e^{2 a_{n}}} \\
\frac{\partial G}{\partial a_{n}}=\frac{10 \log _{10} e^{a_{n}}\left[e^{a_{n}}-\cos \left(\psi-b_{n}\right)\right]}{1-2 e^{a_{n}} \cos \left(\psi-b_{n}\right)+e^{2 a_{n}}} \\
\frac{\partial G}{\partial C}=1
\end{gathered}
$$


To set up a system of equations, one uses these partial derivatives to construct a Jacobian matrix $\mathbf{A}$ whose entries $A_{i, j}$ are

$$
A_{i, j}=\frac{\partial G\left(\psi_{j}, \mathbf{f}\right)}{\partial f_{i}}, \quad\left(i, j=1,2, \ldots, N_{3}\right)
$$

Assuming that the ordering of $\mathbf{f}$ was chosen such that the $a_{n}$ values are followed by the $b_{n}$ values from region I, followed by the $b_{n}$ from region II and then finally $C$, the entries of the matrix would be

$$
\left[\begin{array}{ccccccc}
\frac{\partial G\left(\psi_{1}, \mathbf{f}\right)}{\partial a_{1}} & \ldots & \frac{\partial G\left(\psi_{1}, \mathbf{f}\right)}{\partial b_{1}} & \frac{\partial G\left(\psi_{1}, \mathbf{f}\right)}{\partial b_{N_{1}}} & \ldots & \frac{\partial G\left(\psi_{1}, \mathbf{f}\right)}{\partial b_{N_{1}+N_{2}}} & 1 \\
\vdots & & & & & & \\
\frac{\partial G\left(\psi_{N 3}, \mathbf{f}\right)}{\partial a_{1}} & \ldots & & & & & \\
1 & \ldots & & & & &
\end{array}\right]
$$

where $\psi_{j}$ comes from $\psi$ and $f_{i}$ from $\mathbf{f}$. This is a square matrix and the system of equations can thus be solved for $\delta \mathbf{f}$

$$
\mathbf{A} \delta \mathbf{f}=[\mathbf{g}-\hat{\mathbf{g}}]^{T}
$$

The values found by $\mathbf{f}+\delta \mathbf{f}$ give a better approximation to the desired pattern shape when inserted into eq. 3.32. As before, this pattern can be used as the starting point for a next iteration.

Every iteration alters the location $\psi_{0}$ and the height $G\left(\psi_{0}\right)$ of the shaped beam's peak. This may have to be taken into account during synthesis. Firstly, many shapes require an additional normalization constant $C_{2}$ to be associated with $S(\psi)$. This is needed while $C_{1}$ does not bring $G\left(\psi_{0}\right)$ exactly to $0 \mathrm{~dB}$. If $\max \{G(\psi)\}$ is for example $-1 \mathrm{~dB}$, then $S(\psi)$ should be raised $1 \mathrm{~dB}$ in the next iteration. For shapes without a clear maximum, such as the flat-top pattern (discussed in chapter 4 ), this $C_{2}$ normalization should be omitted. Secondly, for smaller arrays (few nulls), shifting of $S(\psi)$ to follow the $\psi_{0}$ position can be beneficial for the end result.

\section{Halting conditions}

Due to its iterative nature, a halting condition needs to be set for the synthesis procedure. This can be a maximum number of iterations, but usually it is more desirable to stop once a particular constraint for the pattern's shape has been met. In the work of Orchard et al. [91], it is suggested to use the expression $|\mathbf{g}-\hat{\mathbf{g}}|$. The procedure is halted when the largest value in that vector is below some specified maximum. This condition takes both ripple and sidelobe levels into account with similar precision. Sometimes (e.g., in combination with the modifications presented in chapter 5) the ripple converges much faster, while the sidelobes typically do not need to be defined as precise. It may then be worthwhile to stop once the ripple parameters $\left|\mathbf{g}\left[1 \ldots 2 N_{1}+1\right]-\hat{\mathbf{g}}\left[1 \ldots 2 N_{1}+1\right]\right|$ are within the specified bounds. 




FIGURE 3.21 - Root positions

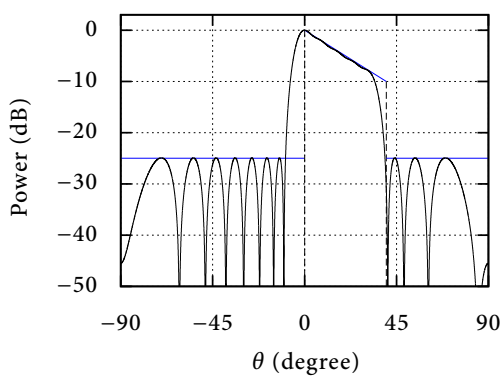

FIGURE 3.22 - Balanced ripple $(\leq 0.1 \mathrm{~dB})$

\subsubsection{EXAMPLE: BALANCED RIPPLE SYNTHESIS}

In the following example, the ripple of the ramp shaped pattern is constrained to $0.1 \mathrm{~dB}$. Similar to the first example, numerical data for several of the iterations performed can be found in appendix A, table A.2.

After having shifted the $S(\psi)$ definition from eq. 3.24 such that its $0 \mathrm{~dB}$ point aligns with $\psi_{0}$ (figure 3.19), the $\mathbf{f}$ vector is assembled. The values in $\mathbf{f}$ are organized as follows: first the $a_{n}$ components of the $N_{1}$ roots, then the $b_{n}$ components of all roots (in order from $-\pi$ to $\pi$ ) and finally the normalization constant $C$

$$
\mathbf{f}=\left[\begin{array}{lllllllll}
0.1 & \ldots & 0.1 & -2.356 & -1.964 & \ldots & 2.356 & 2.749 & -26.234
\end{array}\right]
$$

The $\mathbf{g}$ vector is assembled by first listing the allowed maxima of the ripple, then the minima, and finally the sidelobe heights for region II. Given that the desired height for each sidelobe in region II is set to $-25 \mathrm{~dB}$ again, and the ripple in region I no larger than $0.1 \mathrm{~dB}$

$$
\mathbf{g}=\left[\begin{array}{lllllllll}
0.05 & 0.05 & \ldots & -0.05 & -0.05 & \ldots & -25 & \ldots & -25
\end{array}\right]
$$

The extrema in $G$ and $G-S$ can be found analytically, as suggested in Appendix 9 of [91]. It is important that their locations $\psi$ are assembled in the same order as $\mathbf{g}$.

When the vectors described above are used in eq. 3.41, it will be found that the main peak of the pattern after one iterations is $0.71 \mathrm{~dB}$ below zero. To compensate for this error, one should use $S=S+0.71 \mathrm{~dB}$ in the second iteration and so on. By allowing the main synthesis loop to continue until $|\mathbf{g}-\hat{\mathbf{g}}|<0.1$, the number of iteration performed is 6. The resulting pattern is plotted in figure 3.22. 


\subsection{BEAMPATTERNS FOR TRACKING PURPOSES}

Now that the procedures for synthesis have been established, their capabilities and limitations can be analyzed in order to provide some design guidelines for tracking patterns. A definitive solution will not be given here. All designs in this thesis are driven purely by the desire to keep the tracking algorithm as simple as possible. The null-free shaped region will be considered first, the sidelobe region second.

\subsubsection{ASYMMETRICALLY SHAPED BEAM}

Any shape function $S(\theta)$ that does not return the same value twice on the interval $\left[\theta_{0}, \theta_{1}\right]$ can serve for the purpose of tracking. Since the tracking principle does not change for different $S$-but more importantly because it has not yet been investigated how the shape influences tracking efficiency-the most trivial shape with a unique power-to-position relation is chosen. This is a ramp, defined as

$$
S_{r}(\theta)=\frac{\Delta P}{\Delta \theta} \theta, \quad\left(\theta_{0} \leq \theta \leq \theta_{1}\right)
$$

where $\Delta \theta$ is the width of the shaped region $\left(\theta_{1}-\theta_{0}\right)$ and $\Delta P$ the total power decay over that region. When specifying this $\Delta P / \Delta \theta$ slope, one must consider that

» the power differences associated with a change in elevation angle will be more difficult to detect accurately

» steeper slopes sacrifice more signal strength

In chapter 4 , the case will be made that the ramp shape is only needed for short periods of time. Opting for a relatively steep slope can then be justified, to alleviate the requirements for the measurement equipment. Inspired by the classical $\csc ^{2}(\theta) \cos (\theta)$ example from [91], a decay of $-10 \mathrm{~dB} / 40^{\circ}$ was chosen for the examples shown earlier. This particular shape was defined on a logarithmic scale by

$$
S_{r}(\theta)=-\frac{1}{4} \theta, \quad 0^{\circ} \leq \theta \leq 40^{\circ}
$$

which is mostly convenient for synthesis. The actual (radiation) pattern will not feature this linearity, but is curved slightly concave as depicted by the dashed line in figure 3.23. Depending on how the gain of the beamformer is measured, it might be worthwhile to define the ramp as

$$
S_{r}(\theta)=10^{-\frac{1}{4 \cdot 20} \theta}, \quad 0^{\circ} \leq \theta \leq 40^{\circ}
$$

which is slightly curved in logarithmic scale (figure 3.24), but gives a straight line in the actual beampattern (solid line in figure 3.23). On the other hand, the $\mathrm{dB}(\mathrm{m})$ scale is quite customary for power sensors (e.g., [103]) and a measured quantity can also easily be converted. Later examples will therefore continue to use eq. 3.43. 


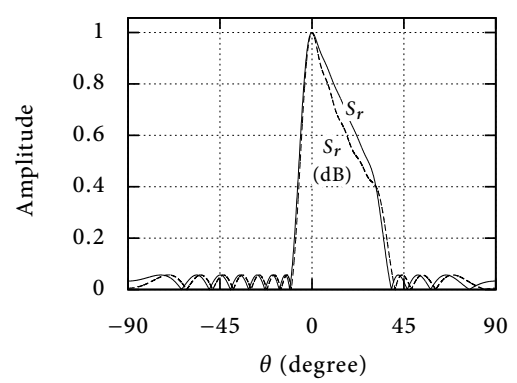

FIGURE $3.23-|F(\theta)|$

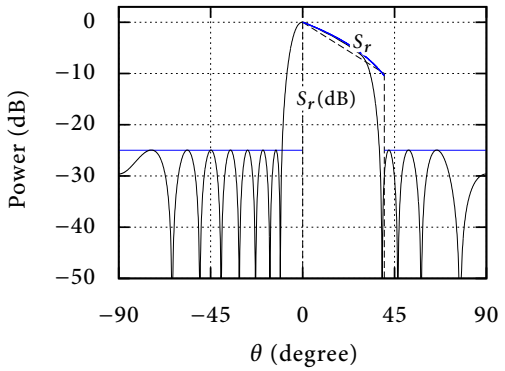

FiguRE $3.24-20 \log _{10}(|F(\theta)|)$

\section{Beamwidth}

Upon closer inspection of the result in figure 3.22, it is found that the beampattern only follows $S_{r}$ for about $30^{\circ}$. Ideally one would like the INBW (chapter 2) of the pattern to match the $40^{\circ}$ range of $S_{r}$; and the shape of the pattern to follow $S_{r}$ exactly between $\theta_{0}$ and $\theta_{1}$. In practice, shaped beampatterns suffer from transient regions [3] (figure 3.25), and the width of the shaped region is primarily dictated by how many roots are moved off the unit circle. Usually a shaped beampattern will therefore turn out narrower than specified, herein indicated by $\left[\tilde{\theta}_{0}, \tilde{\theta}_{1}\right]$ (figure 3.25) or $\Delta \tilde{\theta}=\tilde{\theta}_{1}-\tilde{\theta}_{0}$. Although that is not necessarily problematic for tracking, it should be taken into account that the power actually decays to $-7.5 \mathrm{~dB}$ over a $30^{\circ}$ angle instead of $-10 \mathrm{~dB}$ per $40^{\circ}$.

For wireless communication, the width of the beam is preferred narrow as it relates to the directivity of the array, and thus the overall performance [123]. A narrow beam also requires more effort for accurate tracking (chapter 4 ) and complicates implementation (chapter 5). Nevertheless, in a multi-user environment it is desirable to reduce the beamwidth. For this reason it is useful to know the minimum number of roots needed to create a ramp shape that is fit for tracking purposes. Figure 3.26 depicts the shape of the beam for different numbers of roots $\left(N_{1}=\{3,2,1\}\right)$ moved off the Schelkunoff unit circle. To match the narrower beamwidth, $\left[\theta_{0}, \theta_{1}\right]$ was reduced $10^{\circ}$ per root. Based on this figure it can be concluded that, when region I consists of only one root, it is fairly difficult to recognize a ramp shape. However, as soon as a second root is added, the ramp shape can already be distinguished quite well. With two roots in region $I$, the ramp shaped beampattern of a 16-element $1 / 2 \lambda$ spaced array will feature an INBW of $36.2^{\circ}$. For comparison, the INBW of a conventional beampattern is $14.6^{\circ}$. Hereby it must be noted that the overall sidelobe level of the shaped pattern is much lower than that of a conventional pattern, which also contributes to a wider beam [123]. 


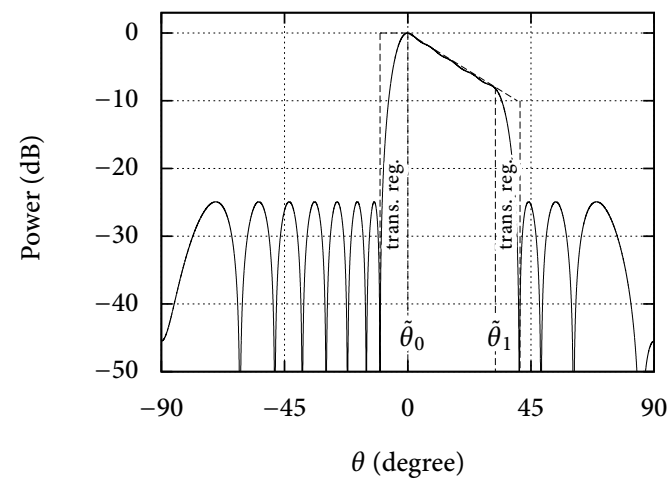

FIGURE 3.25 - Transient regions

\subsubsection{UNIFORM SIDELOBE SUPPRESSION}

An fully adaptive beamformer optimally adjusts its output to the dynamic environment it resides in (\$2.3). If the array excitations are continuously tunable, this may include placement of nulls where strong interference is present. Theoretically it is possible to place nulls during shaped-pattern synthesis. In the case of Orchard-Elliott synthesis, roots can be fixed at a specified location to achieve this [12]. However, two practical reasons prevent dynamic root placement from being feasible for the application considered in this thesis. Firstly, the envisioned in-door infrastructure will be based on switched beamforming. Every pattern needs to be computed a priory and obviously it is not possible to precompute the optimal pattern for all possible situations. Secondly, in spite of Orchard-Elliott synthesis converging relatively fast, it is an iterative procedure and does not produce results fast enough for real-time use.

One way to deal with these limitations is to specify a uniform SLL, as demonstrated by the examples shown so far. A minimal amount of interference suppression is then guaranteed over the entire sidelobe region. From this point of view, the lowest possible uniform SLL is desired. With a 16-element array, the mathematical limit is reached at $-53 \mathrm{~dB}$. Lower numbers of SLL cause numerical instability during synthesis. Using this lower bound is, however, not recommended. In the first place because there are implementation specific aspects to consider (chapter 5 ), but lowering the sidelobes also affects the INBW negatively. Figure 3.27 visualizes the ramp pattern with various sidelobe levels between $-25 \mathrm{~dB}$ and $-40 \mathrm{~dB}$ in steps of $5 \mathrm{~dB}$. With every $5 \mathrm{~dB}$ additional sidelobe suppression, approximately $3^{\circ}$ to $4^{\circ}$ is added to the INBW. As the effect becomes stronger when the common SLL nears its theoretical minimum, subsequent chapters will settle for $-30 \mathrm{~dB}$ SLL. This value is $20 \mathrm{~dB}$ below the lowest point of the proposed ramp, which correspond roughly to the average sidelobe height of a uniformly weighed array of 16 elements. 


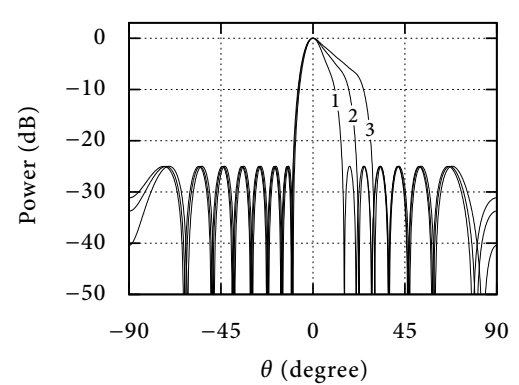

Figure 3.26 - Beamwidth \& roots

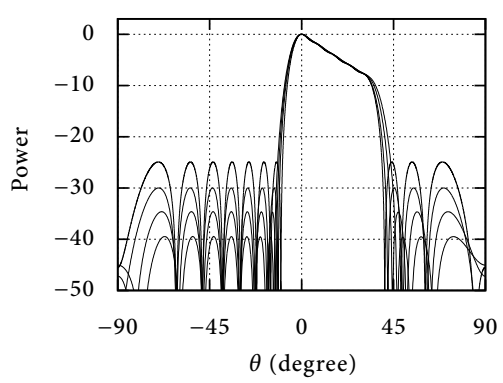

FIgURE 3.27 - Beamwidth \& SLL

\subsubsection{ARray SIZE}

Thus far, the array size has been kept fixed at $N=16$. This number is not arbitrary. From a pattern design perspective, having more antennas is always better. Transition regions will diminish and sidelobes can be suppressed further without severely affecting the beamwidth. Unfortunately, from a commercial and physical realization point of view, the array size cannot be too large. In fact, those considerations demand that it is known how far the array size can be scaled down.

The absolute minimal number of antennas needed for the proposed tracking shape is 4. This number stems from the fact that the total number of roots in eq. 3.10 equals $N-1$. Two of these roots are needed to confine region I, which leaves one to shape the pattern. Figure 3.28 visualizes the corresponding pattern, as well as the $S_{r}$ pattern for other array sizes between $N=4$ and $N=64$. It is clear that the overall performance of the smallest array is far from desirable. Conversely, the influence of transition regions is almost non-existent for 64 antennas. The INBW as well as the directivity (as per \$2.1.4) of the aforementioned array sizes are listed in table 3.2. Based on the trend that can be discovered in the directivity values, arrays of 16 elements are considered to be a good compromise between pattern characteristics and realization implications. The physical size of such an array will be roughly $4 \mathrm{~cm}$ when $d=1 / 2 \lambda$; a modest size that can easily be processed into a commercial product.

TABLE 3.2 - Array size, beamwidth and directivity

\begin{tabular}{cccccc}
$N$ & 64 & 32 & 16 & 8 & 4 \\
\hline INBW & $33.6^{\circ}$ & $38.4^{\circ}$ & $45.2^{\circ}$ & $70.6^{\circ}$ & $120.1^{\circ}$ \\
$\Gamma(\mathrm{dBi})$ & 0.15 & 0.15 & 0.16 & 0.19 & 0.25
\end{tabular}




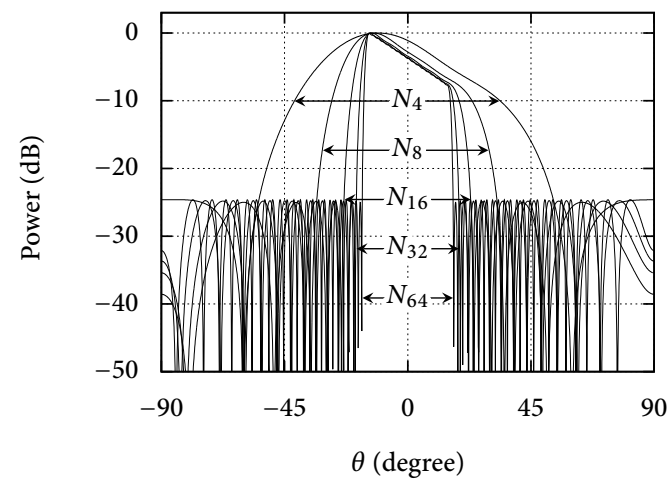

FIGURE 3.28 - Ramp shaped beampattern synthesized for various array sizes

\subsection{RELATED WORK}

A selection of alternative methods for (linear) array pattern synthesis is briefly reviewed next. Although this subject is quite extensive in general, only a handful of techniques can produce shaped beampatterns. The following list of algorithms, although not exhaustive, can be considered 'shaped-pattern synthesis':

» Woodward-Lawson

»Convex optimization

» Alternating projections

" Least squares

» Global optimization

Woodward-Lawson $[132,133]$ is the oldest among synthesis techniques capable of producing null-free patterns. This is, however, the only aspect that still makes it noteworthy. In spite of attempts to improve its performance (e.g. [66]), WoodwardLawson has been superseded on all fronts by Orchard-Elliott synthesis [42]. Convex optimization [77] and the alternating projections method [25] perform similar to Orchard-Elliott in terms of ripple reduction. However, neither exercises the same amount of control over the entire pattern's shape. An advantage over OrchardElliott synthesis is that both approaches can cope with non-equispaced array structures. On the other hand, both techniques are also susceptible to getting stuck in a local optimum. The same holds for least squares synthesis (e.g., [64, 126]), but the ripple produced by the least squares method is not on-par with Orchard-Elliott synthesis.

The global optimization approach to pattern synthesis has been investigated more thoroughly. This highly generic class of algorithms is appealing for their ability to 
manage arbitrary arrays and pattern shapes. Extension to non-ideal- (chapter 5) and planar arrays (chapter 6) would therefore be very straightforward. One of the major drawbacks is the high computational complexity. However, as shapedpattern synthesis is predominantly performed off-line, it is not a major concern here. Literature has given a lot of attention to various biologically inspired algorithms such as the genetic algorithm (GA) $[14,127]$ and particle swarm optimization (PSO) $[22,54]$. Other directions are for example linear programming [51] and simulated annealing (SA) [48]. There are many more variations, but in essence they are based on the same principles.

Due to their popularity, some preliminary testing was conducted on the use of SA [75] and the GA [63] for synthesis of low ripple shaped beampatterns. The particular mechanisms behind both optimization routines will not be explained here. There is comprehensive literature available on this topic [19, 62]. It is, however, important to realize that both the GA and SA search for a solution by making (random) small changes directly to the array excitations, and evaluate the beampattern many times over. This trial-and-error search behavior avoids getting stuck in local optima and allows for arbitrary constraints and arrays. It also makes finding the correct parameters for the algorithm quite hard. Generally the most important parameter for a successful optimization is the cost function to minimize. A popular cost function for pattern synthesis [81] is the root-mean-square of the relative error between the desired and actual pattern shape

$$
E_{Q}=\left(\frac{1}{Q} \sum_{q=1}^{Q} h_{q}\left|e_{q}\right|^{2}\right)^{1 / 2}
$$

computed at $Q$ points in the pattern. Herein, $h_{q}$ is a weight to emphasize various aspects (or areas) of the pattern, and $e_{q}$ is the difference between the objective and actual pattern. The desired pattern shape is defined by a set of masks (i.e., upper and lower bounds) as shown in figure 3.29. For the specific pattern discussed here, the upper bound is given by

$$
F_{\max }(\theta)=\left\{\begin{array}{lr}
-30, & \theta \leq-30^{\circ} \\
0, & -30^{\circ}<\theta<-20^{\circ} \\
-(1 / 4 \times \theta)-5, & -20^{\circ}<\theta<30^{\circ} \\
-30, & \theta \geq 30^{\circ}
\end{array}\right.
$$

and the lower bound by

$$
F_{\min }(\theta)=\left\{\begin{array}{lr}
-\infty, & \theta \leq-20^{\circ} \\
-(1 / 4 \times \theta)-6, & -20^{\circ}<\theta<20^{\circ} \\
-\infty, & \theta \geq-20^{\circ}
\end{array}\right.
$$




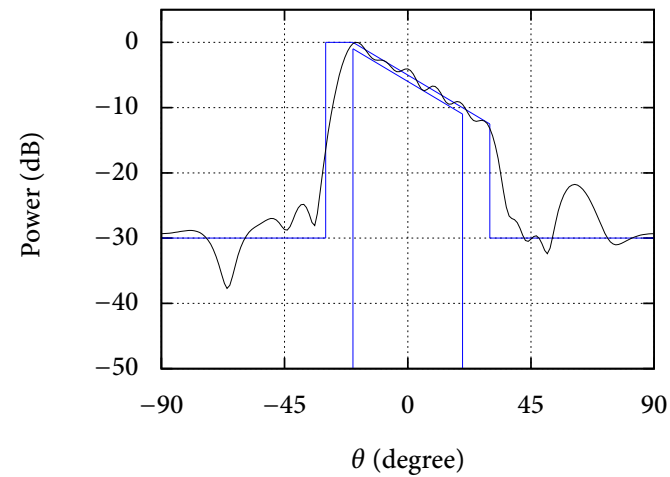

FIGURE 3.29 - Beampattern shaped after $S_{r}(\theta)$ using the real encoded GA of Matlab.

giving a $1 \mathrm{~dB}$ ripple constraint. The relative error at $q$ follows from

$$
e_{q}=\max \left\{\left|F\left(\theta_{q}\right)\right|-\left|F_{\max }\left(\theta_{q}\right)\right|, 0\right\}+\max \left\{\left|F_{\min }\left(\theta_{q}\right)\right|-\left|F\left(\theta_{q}\right)\right|, 0\right\}
$$

One advantage of using upper and lower bounds is that zero costs can be associated with areas of the pattern that produce large errors, but are not directly of interest for the ripple. Examples are the transient regions and the nulls between sidelobes. In the linear array case, such bounds typically result in a pattern as depicted in figure 3.29. This particular result was obtained with the Matlab Global Optimization Toolbox, using a real encoded GA optimization (to avoid the quantization errors made by binary encoding). The chromosomes were encoded, as described in [127], by a vector whose entries are alternatively the real and imaginary parts of $I_{n}$. A population size of $6 \mathrm{~N}$ was chosen with six elites per generation, and a crossover fraction of 0.8 . Termination was forced after the $10000^{\text {th }}$ generation and clearly the optimizer did not succeed in finding a pattern within the bounds of eq. (3.46) and (3.47). In an attempt to improve the result, the cost function [81]

$$
E_{Q}=\sum_{q=1}^{Q} \log \left|\frac{S_{q}}{F_{q}}\right|
$$

as well as a hybrid of eq. 3.45 and eq. 3.45 [127] were also evaluated. Herein, $S_{q}$ is the desired power level at $q$ and $A F_{q}$ the actual power. Additional experimentation to improve the ripple included using absolute relative errors and square errors; increasing and decreasing the population size; performing several short runs instead of a longer one; and more dense sampling $(q)$ where $F_{\min } \neq-\infty$. None of it gave significant improvement over figure 3.29. Simulated annealing was found to perform similar to the genetic algorithm in terms of ripple reduction. 


\subsection{Conclusions}

This chapter presented power pattern synthesis for linear arrays, with an analytical foundation. One such technique, Orchard-Elliott synthesis, is recommended for the synthesis of beampatterns used in shaped-pattern tracking/DoA estimation. With an array of 16 antennas, beampatterns produced by Orchard-Elliott synthesis can approximate the specification with a ripple of $0.1 \mathrm{~dB}$ or less. The analytical approach does limit the allowed array configurations to uniformly spaced- and identically orientated antennas. However, for the application envisaged in chapter 1 (i.e., a ceiling mounted access point), this is not considered to be a problem. Moreover, the synthesis algorithms compatible with arbitrary arrays have been found less suitable. Experiments with genetic algorithms and simulated annealing did not lead to the desired beampattern shape with less than $1 \mathrm{~dB}$ (ripple) error. The importance of reducing this ripple shall become evident in the next chapter, where DoA estimation and tracking based on the shape of beampatterns is explained in more detail.

The shape selected for tracking and DoA estimation is a ramp with $-10 \mathrm{~dB}$ power decay per $40^{\circ}$. Such a ramp features the most trivial-but unique-relation between elevation angle and power, which helps to keep the tracking algorithm simple. At roughly $45^{\circ}$, the obtained beam is fairly wide. In the next chapter this is shown to be convenient for tracking, but it also sacrifices some of the directivity gained by beamforming. Depending on the array size, it may be possible to reduce the beamwidth. Sixteen elements is the recommended size based on performance characteristics such as directivity. A beamwidth of $36^{\circ}$ could then also be obtained. All sidelobes of the shaped pattern are suppressed uniformly to $-30 \mathrm{~dB}$, to compensate the lack of null-steering (for interference suppression) in single output beamformers. 



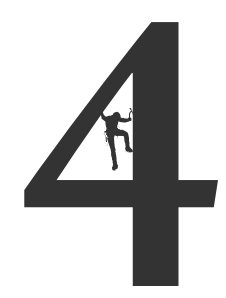

\title{
TRACKING BY MEANS OF SHAPED BEAMPATTERNS
}

\begin{abstract}
When a beamforming network is implemented entirely in the analog domain, and its output is the only source for signal processing, the shape of the beampattern can be exploited for DoA estimation. In this chapter, a tracking technique is developed based on that principle. It requires the use of an asymmetrically shaped beampattern, and a pattern which features a flat response. Correct steering is achieved by considering the difference in power collected from the different beampatterns.
\end{abstract}

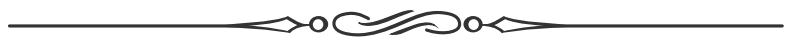

$\mathrm{C}$

OMPARED to their analog counterpart, substantial more effort has been put into the development of adaptive antenna arrays that operate on digital data, obtained by sampling signals from the individual antenna elements $(\$ 2.3)$. This is not surprising given their enormous flexibility [55]. However, as carrier frequencies increase, the number of antennas increases, and bandwidths become larger, the multitude of RF components (in particular A/D converters [121]) needed for a digital beamformer tends to become expensive and energy hungry. The fully analog beamformer combines all signals before they are sampled, and is more attractive in that respect [33]. On the other hand, it is much less flexible.

Given the requirements discussed in chapter 2, scan-based tracking was identified as the sole means to cope with the limitations of such a single output beamformer. Scanning is conceptually easy and it also matches well with RoF-based integrated OTTD beamforming as discussed in chapter 1 . However, it has some drawbacks in the setting of a wireless communication system, that are primarily caused by the need for probing various scan angles. To mitigate the associated high degree of beam misalignment, chapter 2 proposed to replace the conventional beam by

Parts of this chapter have been published in [TB:5]. 
a ramp shaped beam. Now that the methods for synthesis of such a beampattern have been established in chapter 3, a suitable shaped-pattern tracking mechanism is developed next.

Recall from chapter 2 and $\$ 3.4 .2$ that dynamic interference suppression by null placement is highly impractical in the context of switched beamforming. Hence, the primary objective in this chapter will be aligning the main beam with the angle of incidence. This problem is approached from the receiver side, whereby it is assumed that the transmitter is omnidirectional. Comprehensive protocols [128] are needed when both the receiver and transmitter perform beamforming, which will not be considered here. When it is also assumed that the power density is sufficiently constant with respect to the receiver's sample time, the known unique power-to-elevation-angle relation of the ramp shaped power pattern can be used to steer the beam immediately in the right direction. The actual DoA does not need to be known precisely to do this. Tracking $(\$ 4.2)$ and DoA estimation $(\$ 4.3)$ are therefore treated separately.

\subsection{Relative POWER}

Omitting the fact that the output of the beamformer needs to be calibrated in order to know what power level corresponds to the peak of the ramp shaped pattern from chapter 3 , measuring absolute power is not sufficient for tracking or DoA estimation. The shape of the beampattern is not the only factor that influences the received power. Consider for example the additive path loss according to Friis, caused by increasing the distance between the array and peer device from $1.5 \mathrm{~m}$ to $2 \mathrm{~m}$

$$
10 \log _{10}\left(\frac{4 \pi 1.5 \mathrm{~m}}{0.005 \mathrm{~m}}\right)^{2}-10 \log _{10}\left(\frac{4 \pi 2 \mathrm{~m}}{0.005 \mathrm{~m}}\right)^{2} \simeq-2.5 \mathrm{~dB}
$$

Although this is an overly simplified example, it illustrates that path loss will be in the same order of magnitude as a change of elevation angle based on the $-10 \mathrm{~dB} / 40^{\circ}$ ramp shaped pattern (identified by $\mathcal{L}_{r}$ from here on). Translational motion (i.e., changing distance between mobile device and array) could easily be mistaken for angular displacement (i.e., changing angle between mobile device and array center). This is problematic while the steering angle must only be updated when the angle of incidence changes. In case of translational motion, steering will yield no improvement. On the contrary, the steering angle should be maintained to prevent further signal degradation. Besides path loss, there are many more phenomena which can influence the received power. Fading [105], polarization mismatch and blocking are just a few examples of what needs to be taken into account. Essentially one would like to take the entire link budget [95] out of the equation, except for the beampattern's shape. The most effective way to do this is by measuring the power relative to a differently shaped beampattern. 


\section{Flat-topped beampattern}

let $\mathcal{L}_{r}$ be synthesized with its MRA positioned at $0^{\circ}$, and it overall shaped defined by

$$
S_{r}(\theta)=\left\{\begin{array}{lr}
-30, & \theta<\theta_{0} \\
-1 / 4 \theta-5, & \theta_{0} \leq \theta \leq \theta_{0} \\
-30, & \theta>\theta_{1}
\end{array}\right.
$$

Then the second shape, used to relate with $\mathcal{L}_{r}$, is given by

$$
S_{f}(\theta)=\left\{\begin{array}{rrr}
-30, & \theta<\theta_{0} \\
0, & \theta_{0} \leq \theta \leq \theta_{0} \\
-30, & \theta>\theta_{1}
\end{array}\right.
$$

If synthesized with $\theta_{0}=-20^{\circ}$ and $\theta_{0}=20^{\circ}$ (according to the procedures discussed in chapter 3$)$ this definition gives in a flat-topped beampattern $\left(\mathcal{L}_{f}\right)$, as depicted next to the ramp pattern in figure 4.1. Note that the shaped beam overlaps the ramp entirely, and that its response in terms of power is almost unaffected by angular displacement within the shaped $\left[\tilde{\theta}_{0}, \tilde{\theta}_{1}\right]$ region. The difference in power as a result of alternating between $\mathcal{L}_{f}$ and $\mathcal{L}_{r}$ can therefore distinguish variation caused by angular motion from all other effects.

The $\mathcal{L}_{f}$ pattern also provides a convenient reference point for the absolute value that can be expected at the peak of $\mathcal{L}_{r}$. By interpreting the ramp power relative to that of $\mathcal{L}_{f}$, it will implicitly be normalized to the $0 \mathrm{~dB}$ point. Comprehensive calibrations are thus avoided. The only requirement is that the peer device resides somewhere in the $\left[\tilde{\theta}_{0}, \tilde{\theta}_{1}\right]$ sector covered by the shaped beams.

\section{Power-to-DoA relation}

Assuming that the device to be tracked is indeed located between $\tilde{\theta}_{0}$ and $\tilde{\theta}_{1}$, let the power from $\mathcal{L}_{f}$ be denoted by $P_{f}$ and the power of $\mathcal{L}_{r}$ by $P_{r}$. The minimum ramp power $P_{\min }=\mathcal{L}_{r}\left(\tilde{\theta}_{1}\right)$ is then a known value relative to the maximum power $P_{\max }=\mathcal{L}_{r}\left(\tilde{\theta}_{0}\right)=\mathcal{L}_{f}$. By approximation, the angular position relative to $\tilde{\theta}_{0}$ can be found with

$$
\theta_{r}=\frac{\left|P_{f}-P_{r}\right|}{\left|P_{\max }-P_{\min }\right| /\left(\tilde{\theta}_{1}-\tilde{\theta}_{0}\right)}
$$

In the case of figure 4.1, this amounts to $\theta_{r}=\left|P_{f}-P_{r}\right| /(7.5 / 30)$. Since the $\tilde{\theta}_{0}$ angle will be known from synthesis and the applied steering angle, the absolute DoA is given by $\tilde{\theta}_{0}+\theta_{r}$. 


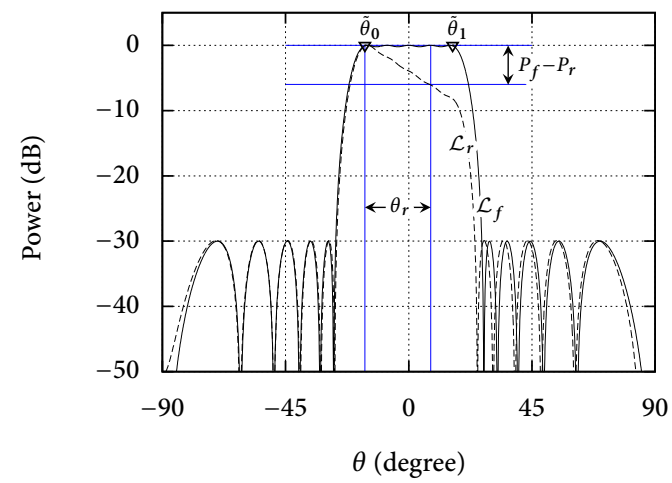

FIGURE 4.1 - Flat $\left(\mathcal{L}_{f}\right)$ and $\operatorname{ramp}\left(\mathcal{L}_{r}\right)$ shaped beampatterns

The objective of tracking is to ensure that the shaped beam remains aligned with the mobile device's angular position during wireless communication. Because a flat-topped beampattern is used, this can be defined as keeping the DoA between $\tilde{\theta}_{0}$ and $\tilde{\theta}_{1}$ (figure 4.1). The $\theta_{r}$ angle given by eq. 4.4 does not need to be known precisely for this. Due to its flat power response, it is also sufficient if the $\mathcal{L}_{f}$ pattern is simply steered slightly to the left or right based on whether $P_{r}$ gets too close to $P_{\max }$ or $P_{\min }$ respectively. Although technically this could be seen as a rough form of DoA estimation, this chapter makes a distinction between tracking and actual DoA estimation. Viz., when accurate estimates of the DoA are desired, eq. 4.4 will not always suffice and the power-to-angle relation needs to be characterized more precisely for different steering angles. An elaborate discussion on this subject will follow in $\$ 4.3$. A tracking mechanism based on $\delta P=\left|P_{f}-P_{r}\right|$ is presented first.

\subsection{TRACKING}

For any tracking mechanism derived from eq. 4.4, the beamformer must alter the shape of its beampattern between $\mathcal{L}_{f}$ and $\mathcal{L}_{r}$, and $P_{f}$ and $P_{r}$ must respectively be measured. The $\mathcal{L}_{f}$ and $\mathcal{L}_{r}$ patterns do not need to be used the same amount of time. It is actually beneficial for energy efficiency to change the shape of the beampattern as little as possible. However, there is need for a fixed ratio in order to be able to correct the steering angle in time. Let this ratio be defined in a parameter

$$
\Upsilon=\frac{\# \text { iterations } \mathcal{L}_{f}}{\# \text { iterations } \mathcal{L}_{r}}
$$

Given that the flat-topped pattern sacrifices less gain, the algorithm presented in this section will use that shape as the default. The general idea is to employ $\mathcal{L}_{f}$ for 
a (longer) period of time; switch to $\mathcal{L}_{r}$ for a short(er) period to determine if the DoA is close to either $\tilde{\theta}_{0}$ or $\tilde{\theta}_{1}$; update the steering angle of both patterns when necessary; then switch back to $\mathcal{L}_{f}$ and repeat. The $\Upsilon$ parameter can be used to tweak this cycle to track faster movement, or save energy by not altering the pattern shape for a longer period of time. Hereby it must be noted that effects from e.g. path loss and fading will only be nullified if the interval between measuring $P_{f}$ and $P_{r}$ is small enough. Subsequently, on the basis of figure 4.1, increasing values of $\delta P=\left|P_{f}-P_{r}\right|$ indicate positive angular displacement while a decreasing difference means negative angular displacement. The patterns are steered (to the left) when $\delta P$ is close to $0 \mathrm{~dB}$, or (to the right) when close to $7.5 \mathrm{~dB}$. In contrast to conventional beam scanning (\$2.3.3), there is thus no need for anticipatory steering, and there will be little risk of the signal's angle of incidence ending up in a null which would disrupt communication.

Before considering the details of this algorithm, some assumptions will be made. First and foremost, the aspects covered in this chapter concern only the tracking of an individual mobile wireless device that does not utilize beamforming on the transmission side. Secondly, the mobile wireless device is assumed to be localized successfully (i.e., finds itself somewhere in $\left[\tilde{\theta}_{0}, \tilde{\theta}_{1}\right]$ ) before the tracking loop is initialized. This could for example be accomplished with sector scanning, as described in IEEE 802.15.3C [67]. The third requirement is that the transmitted power must be fairly constant over time, so that the received power depends solely on the device's position. Lastly, only line of sight (LoS) channels are considered. Once the conditions are met and the tracking loop is started, essentially three operations are iteratively performed: 1 ) normalization, 2) determination of the steering-direction and 3 ) conditional steering.

\subsubsection{Normalization}

Within a dynamic environment it is important that $P_{f}$ is used as a reference, and that tracking is based on the difference between $P_{f}$ and $P_{r}$. The time between the measurements can therefore not be too long. Yet, power is typically acquired by averaging. Let a single measurement therefore take up a particular amount of time $\tau_{p}$. The aforementioned $\Upsilon$ parameter expresses the duration of employing $\mathcal{L}_{f}$ by integer multiples of $\tau_{p}$. Each tracking cycle then consists of $\Upsilon \cdot \tau_{p}$ time slots in which the $\mathcal{L}_{f}$ pattern is used, followed by a single $\tau_{p}$ slot of $\mathcal{L}_{r}$ use. $P_{f}$ should be measured in the $\Upsilon^{\text {th }}$ time slot, $P_{r}$ in the $(\Upsilon+1)^{\text {th }}$. The first $\Upsilon-1$ periods will thus be ignored by the tracking algorithm.

\subsubsection{Trigger AND Direction}

Steering of the beampatterns is triggered whenever $\delta P$ indicates that the device being tracked is about to leave $\left[\tilde{\theta}_{0}, \tilde{\theta}_{1}\right]$. A safety margin of e.g. $1 \mathrm{~dB}$ could be incorporated for robustness. In that case, the pair of patterns from figure 4.1 would be steered whenever their relative power difference is less than $1 \mathrm{~dB}$ or exceeds $\left|P_{\max }-P_{\min }\right|-1$. The corresponding directions are fairly evident from the shape of 
the ramp. In figure 4.1, it will be negative if $\delta P<1 \mathrm{~dB}$ and positive when $\delta P>6.5 \mathrm{~dB}$. However, it is proposed that the slope of the ramp is reversed when its MRA lies to the left of $\theta=0^{\circ}$. The reasoning behind this is two-fold

"It leads to an implementation specific advantage (smaller amplitude range)

»It entails more consistent usage of the shapes with planar arrays

Elaborate explanations of both arguments are postponed until chapters 5 and 6 respectively. Here one only needs to be aware that, once the ramp shape is reversed, the roles of $P_{\max }$ and $P_{\min }$ will switch with respect to the steering directions.

\subsubsection{STEERING}

Having reached one of the conditions to steer, the tracking algorithm must determine where to position the beam. If a beamformer is continuously tunable, the ideal steering angle $\theta_{d}$ could be determined by eq. 4.4. Switched-OTTD beamforming only supports a finite set of array excitations [94, 98]. Consequently, a set of beampatterns needs to be predefined at design time. Various implementation aspects will be discussed in chapter 5. For the sake of generality, the excitations corresponding to the desired beampattern sets are first assumed to be stored in a lookup table $\mathbf{T}$. Let $\mathbf{T}_{r}$ define the sets of excitations which generate $\mathcal{L}_{r}$, subject to different (discrete) steering angles, and $\mathbf{T}_{f}$ the excitation sets for $\mathcal{L}_{f}$.

\section{Steering shaped patterns}

Actual steering of shaped beampatterns is not different from conventional patterns. One possible caveat is that there is not always a clear MRA by which the steering angle can be defined. For $\mathcal{L}_{f}$ and $\mathcal{L}_{r}$, the half-way point between the nulls at $b_{0}$ and $b_{N_{1}}$ (that enclose the shaped region) is chosen as the MRA. When expressed in standard notation (chapter 2), the pattern is steered by weighing every complex $\stackrel{\circ}{n}_{n}$ value as follows

$$
I_{n}=\stackrel{\circ}{I}_{n} \cdot e^{-j k d \sin \left(\theta_{d}\right)}
$$

Herein, it is assumed that the MRA of the pattern aligns with $0^{\circ}$ before steering (i.e., synthesized as such), so that it will arrive at the specified angle $\theta_{d}$. In terms of the Schelkunoff polynomial representation (chapter 3 ), the same result is obtained by swiveling the roots $\stackrel{\circ}{w}_{n}$ in the complex plane around the unit circle by a common angular distance $\delta \psi$

$$
w_{n}=\stackrel{\circ}{w}_{n} \cdot e^{-j \delta \psi}
$$

where $\delta \psi$ is determined by the distance between $\psi=0$ and $\psi_{d}=k d \sin \left(\theta_{d}\right)$. A detailed description of this method was given in \$3.1.1. The advantage of eq. 4.7 is that the roots still need to be converted into excitations, which gives the designer more freedom during the implementation phase. 


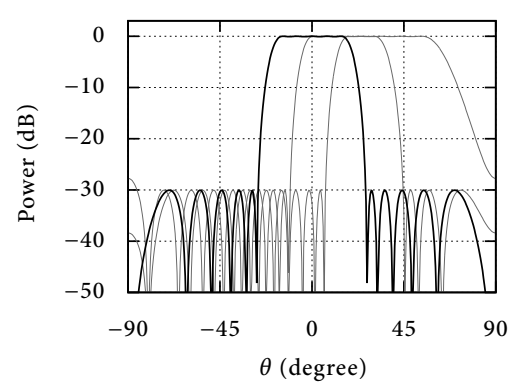

FIGURE 4.2 - Steered flat-topped pattern

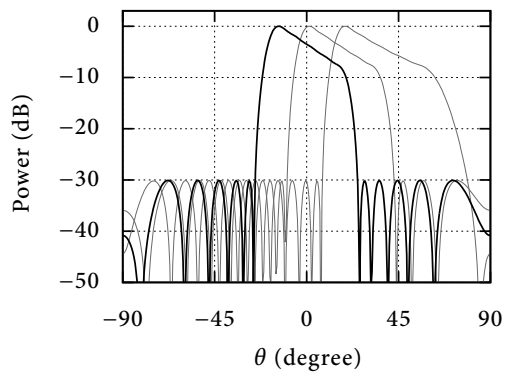

FIGURE $4 \cdot 3$ - Steered ramp pattern

\section{Scan angles selection}

For practical reasons, $\mathbf{T}_{f}$ and $\mathbf{T}_{r}$ cannot be too fine-grained in case each relative phase $\left(\angle I_{n}\right)$ is realized with a dedicated OTTD line. In that case there is a direct trade-off between the separation of two adjacent scan angles and the complexity of the beamformer design. Each additional scan angle requires $N$ (i.e., the number of channels in the beamformer) additional delays to be fabricated. On the other hand, the scan angles should be able to cover a particular (chosen) sector entirely without gaps.

Considering the above, a flat-topped beampattern suits switched beamforming quite well. Due to its flat power response, the scan angles can be chosen more coarse than for a conventional beam. Sweeping a conventional beam over a particular sector results in cusping loss (\$2.3.1) between two adjacent MRA steering angles. The signal strength will be weaker here. To reduce these losses, one would have to increase the number of scan angles. As seen in figure 4.2, with a flat-topped pattern this issue does not arise. Principally the entire visible region could be covered with just six scan angles, however, some overlap is desired. Overlap provides an easy means to prevent erratic steering behavior (i.e., oscillation between two adjacent steering angles). Although this problem could also be tackled by control theory [111], that subject is considered to be outside the scope of this work. Two adjacent patterns are given a convenient overlap of $\left(\tilde{\theta}_{1}-\tilde{\theta}_{0}\right) / 2$, which means that the steering angle is incrementally increased in steps of $\Delta \theta= \pm 15^{\circ}$ for the proposed $\mathcal{L}_{f}$ and $\mathcal{L}_{r}$ patterns. Given the triggers explained in $\$ 4.2 .2$, steering left or right will then roughly bring the pattern's MRA back to the peer device's last known position. The resulting overlap can be seen in figures 4.2 and 4.3 for several positive scan angles.

\section{Maximum angle}

Theoretically, with an element spacing of $1 / 2 \lambda$, both shapes could continue to be steered until $\theta_{d}= \pm 90^{\circ}$. However, it is well known that (owing to a reduced effective aperture [6o]) the shape of the beampattern in the far field generally changes as it is steered further away from broadside. With shaped beampatterns, this change 
is predominantly experienced as broadening of the $\left[\tilde{\theta}_{0}, \tilde{\theta}_{1}\right]$ shaped region. The maximum steering angle is limited because of this effect. There are different criteria that could be used to define such a maximum steering angle. One is that spatial aliasing of the shaped beam, which can for example be seen clearly in figure 4.8, should not be allowed. When this is the condition, the steering angles for $\mathcal{L}_{f}$ and $\mathcal{L}_{r}$ must stay confined between $\pm 35^{\circ}$ with a 16-element array. Due to a broader $\Delta \tilde{\theta}$, when steered $35^{\circ}$ away from broadside, the pair of shaped beampatterns can then cover a sector of roughly $120^{\circ}\left(\left[-57^{\circ}, 57^{\circ}\right]\right.$ to be precise $)$. Another selection criterion could be the linearity of the $\mathcal{L}_{r}$ slope. Because this property is mostly relevant for actual DoA estimation, it will be discussed in \$4.3. Combining the recommended $15^{\circ}$ overlap with a maximum steering angle of $35^{\circ}$ gives the following selection of relevant scan angles

$$
\left\{-35^{\circ},-30^{\circ},-15^{\circ}, 0^{\circ}, 15^{\circ}, 30^{\circ}, 35^{\circ}\right\}
$$

However, as the $\pm 35^{\circ}$ and $\pm 15^{\circ}$ angles make $\pm 30^{\circ}$ redundant, the final selection for the upcoming sections is

$$
\left\{-35^{\circ},-15^{\circ}, 0^{\circ}, 15^{\circ}, 35^{\circ}\right\}
$$

Let $\mathbf{T}_{r}(i)$ select the excitations for the ramp shaped pattern steered to position $i$ and $\mathbf{T}_{f}(i)$ the flat pattern subjective to the same angle. It is assumed that $i=0$ selects the pattern steered to $-35^{\circ}, i=1$ to $-15^{\circ}$ etc. Steering is then accomplished by updating $i$ to $i-1$ or $i+1(0 \leq i \leq 5)$. When the device being tracked moves out of the array's reach (i.e., further than approximately $60^{\circ}$ from broadside), a hand-off procedure is required to select a nearby antenna array in the network [20].

\subsubsection{Simulations}

Verification of the proposed tracking mechanism has been carried out by means of simulations in Matlab, using lookup tables as explained above. The performed simulations mimic LoS $60 \mathrm{GHz}$ RF transmission between an individual mobile device and a ceiling mounted 16 -element $(1 / 2 \lambda)$ equispaced linear array. This is assumed to be within a small in-door scenario for which a single antenna array, that covers a sector of $120^{\circ}$, is sufficient. Using a rudimentary link budgeting as presented in e.g. [129], the simulated wireless channel comprises transmit gain, path loss and receive gain. In this case, the receive gain is given by the differently shaped beampatterns. Path loss is approximated by the loss exponent model

$$
\mathrm{FSPL}_{\mathrm{dB}}=10 \log _{10}\left(\frac{4 \pi d}{\lambda}\right)^{\eta}
$$

wherein $\eta$ is 1.55 for short-range LoS connections [71], and the transmit gain is based on a $60 \mathrm{GHz}$ microstrip patch antenna model, exported from CST microwave 




FIGURE 4.4 - Gain of a $60 \mathrm{GHz}$ patch antenna model from CST

studio [35]. Figure 4.4 visualizes the latter. As one would expect from a mobile device, both the $(\phi, \theta)$ orientation of this antenna's gain, and its position relative to the array were changed over time. The (angular) position of the antenna was determined by a random walk between $-45^{\circ}$ and $45^{\circ}$. The distance between the device and ceiling was varied between $1.5 \mathrm{~m}$ and $2.2 \mathrm{~m}$ in a similar fashion. Such boundaries could be expected if the array were to be mounted on the ceiling of a typical small office space (e.g., $3 \mathrm{~m}$ height). Transmit power was set to $16 \mathrm{dBm}$ based on the commercial products reported in [114].

The transmitted signal is an orthogonal frequency devision multiplexing (OFDM) signal in case of Nyquist sampling, such that it can be modeled as white gaussian noise (WGN). When sub carriers are modulated by QPSK or QAM, the sustainable SNR (at short range) is between $14 \mathrm{~dB}$ and $18 \mathrm{~dB}$ (e.g., $[45,101]$ ), given a BER of $10^{-4}$. The overall channel was therefore subjected to additive WGN of $14 \mathrm{~dB}$.

The received power follows from averaging the sampled signal's amplitude, after having passed the beamformer. A chain as depicted in figure 4.5 is hereby assumed, whereby it must be mentioned that there should be no interference of automatic gain controllers. In the theoretical sense, power as defined by

$$
P=\frac{1}{\tau_{p}} \int_{0}^{\tau_{p}}|y(t)|^{2} d t
$$

then determines the final values on which steering of the beampattern relies. A longer sampling time will thus help reduce the influence of external factors. On 


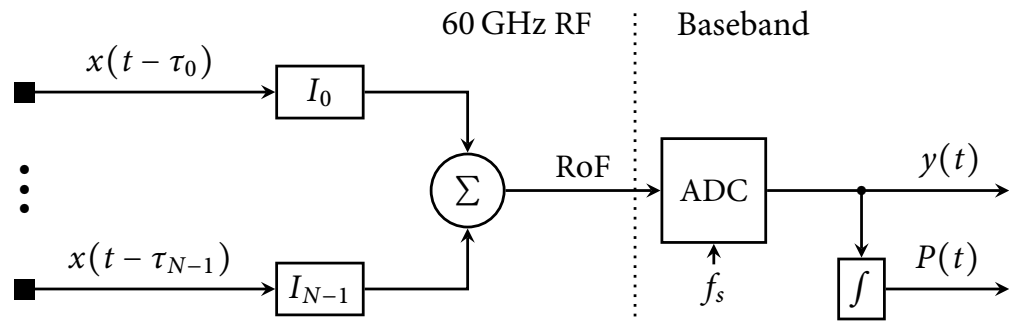

FigURE 4.5 - Simulated power measurement

the basis of a power acquisition time used in commercial sensors [103], $\tau_{p}$ was set to $48 \mu \mathrm{s}$. In a practical scenario, averaging takes place over $K$ baseband samples

$$
P=\frac{1}{K} \sum_{1}^{K}|y(k)|^{2}
$$

In IEEE $802.15 .3 \mathrm{C}$, the HSI reference sampling rate $f_{s}$ is $2.68 \mathrm{GHz}$ [67]. At this frequency, roughly 128000 samples could be collected for a measurement in the given amount of time. Given that the time required to change the shape of the pattern is in the order of sub-microseconds [98] (for optical switched delay lines), the provided parameters are considered to be a realistic choice.

Figure 4.6 shows a simulation trace under the circumstances described above. The angular position of the device has been plotted as the horizontal solid line at $0 \mathrm{~dB}$,

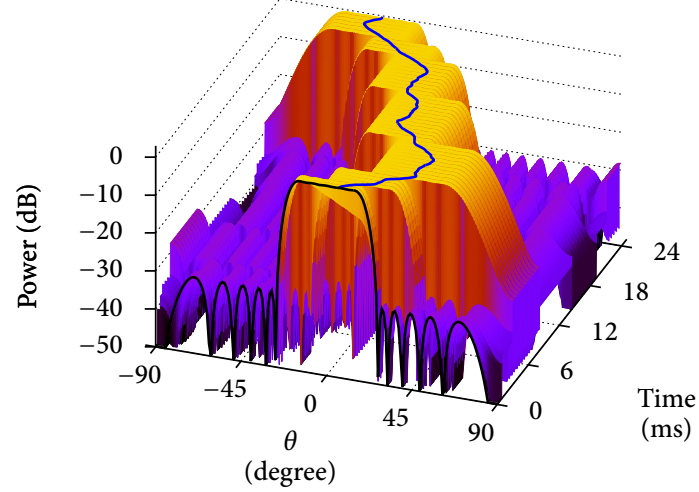

FiguRE 4.6 - Tracking with a $40^{\circ}$ shaped beam 
together with the shape of the beampattern as a function of time. Note that the flat-topped pattern is dominant (i.e., the ramp is not visible) because of the $\Upsilon$ parameter (chosen 10 here). For practical reasons, the trace only shows $500 \tau_{p}$ slots. The corresponding dynamic behavior can be considered extreme for this $24 \mathrm{~ms}$ time frame $(>120 \mathrm{~m} / \mathrm{s})$, but it can be observed that the beam follows the position of the device as desired. Based on the condition that the angular position of the device is never found to be outside the shaped region $\left(\left[\tilde{\theta}_{0}, \tilde{\theta}_{1}\right]\right)$, Monte Carlo simulation shows that $\Upsilon$ must be set to 10 or less in this extreme scenario. In a more realistic scenario (not shown here), $\Upsilon$ can be increased by at least an order of magnitude to save energy. This assumes the average human walking speed of $1.4 \mathrm{~m} / \mathrm{s}$.

\section{Narrow beam}

Chapter 3 demonstrated that the shaped beampatterns can be synthesized much narrower to achieve higher directivity. Employing 16 antennas, $\mathcal{L}_{f}$ and $\mathcal{L}_{r}$ could be obtained with an INBW of roughly $20^{\circ}$. Driven again by alias free steering, the maximum scan angles of these patterns are $\pm 44^{\circ}$. The predefined beam positions will be separated by $7.5^{\circ}$, which means from an implementation point of view that more than twice as many scan angles need to be generated. From a tracking point of view, one can see in figure 4.7 the expected increase in dynamic behavior.

\subsubsection{Ripple tolerance}

Chapter 3 was largely devoted to ripple reduction in the shaped region. Although the influence of the ripple will only be measurable directly by performing DoA estimations, to some extent it also determines whether a mobile device can be

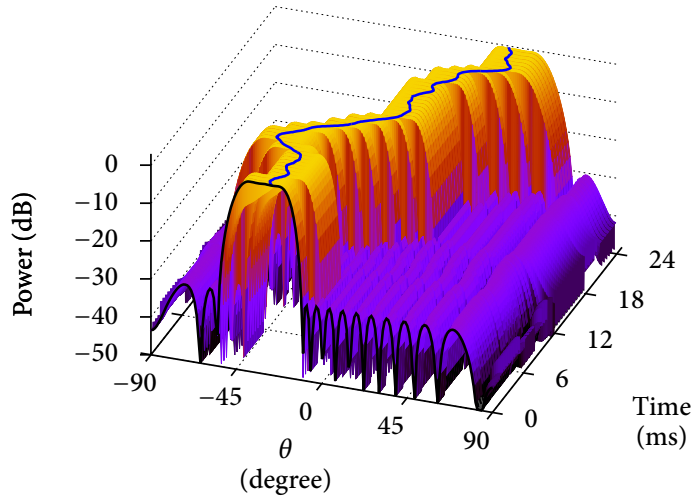

FIGURE 4.7 - Tracking with a narrower $\left(20^{\circ}\right)$ beam 
tracked successfully. The latter depends strongly on how the margins for $P_{\min }$ and $P_{\max }$ are chosen. Based on the simulations describe before, both margins must be set to roughly $0.5 \mathrm{~dB}$ for error free tracking with $0.1 \mathrm{~dB}$ ripple. Assuming such margins, the algorithm can sustain correct operation with a ripple of $1.5 \mathrm{~dB}$. A $2 \mathrm{~dB}$ ripple frequently leads to steering errors. The ripple should thus not exceed $1.5 \mathrm{~dB}$ for tracking purposes.

\subsection{DIRECTION-OF-ARRIVAL ESTIMATION}

From the previous section it may be concluded that tracking based on shaped beampatterns can be made quite robust. Actual DoA estimation will be much more susceptible to changes in the environment and imperfections in the patterns. In the remainder of this chapter it is investigated how well the (angular) position of a peer device can be estimated from shaped beampatterns.

\subsubsection{SLOPE CHARACTERIZATION}

As the MRA of a shaped beam is steered away from broadside (\$4.2.3), its shaped region will inherently broaden. If the DoA is to be estimated based on the shaped region, this broadening needs to be taken into account. For the relatively small steering angles chosen in $\$ 4.2 .3$, broadening can by approximation be considered linear, such that only the $\tilde{\theta}_{0}$ and $\tilde{\theta}_{1}$ values in the denominator of eq. 4.4 need to be adjusted to the steering angle. To this end, $1 / 2 \lambda$ spacing is assumed; then the $\psi$-space angle corresponding to $\tilde{\theta}_{0}$ is given by $\tilde{\psi}_{0}=\pi \sin \left(\tilde{\theta}_{0}\right)$. In the linear $\psi$-space, when the ramp shape is not reversed (\$4.2.2), the lowest point of the ramp will be located a fixed distance (regardless of the steering angle) to the right of $\tilde{\psi}_{0}$. This distance is given by $\pi \sin (\Delta \tilde{\theta})$, where $\Delta \tilde{\theta}$ was defined as $\tilde{\theta}_{1}-\tilde{\theta}_{0}$ in $\$ 3.4 .1$. For the main example chosen in this thesis, it holds that $\Delta \tilde{\theta}=30^{\circ}\left(\$_{3.4 .1}\right)$. The $\theta$ equivalent of this point (i.e., $\tilde{\theta}_{1}$ ) depends on the location of $\theta_{0}$. The larger the steering angle, the further away $\tilde{\theta}_{1}$ will be from $\tilde{\theta}_{0}$. When $\tilde{\theta}_{1}>\tilde{\theta}_{0}$, by eq. 3.7 this can be expressed as

$$
\begin{aligned}
\tilde{\theta}_{1}\left(\tilde{\theta}_{0}\right) & =\sin ^{-1}\left(\frac{\tilde{\psi}_{0}+\pi \sin (\Delta \tilde{\theta})}{\pi}\right) \\
& =\sin ^{-1}\left(\frac{\pi \sin \left(\tilde{\theta}_{0}\right)+\pi \sin (\Delta \tilde{\theta})}{\pi}\right) \\
& =\sin ^{-1}\left(\sin \left(\tilde{\theta}_{0}\right)+\sin (\Delta \tilde{\theta})\right)
\end{aligned}
$$

The reversed $\operatorname{ramp}\left(\tilde{\theta}_{0}>\tilde{\theta}_{1}\right)$ follows similarly, except that the result will be negative. For the steering angles presented earlier, table 4.1 lists the corresponding $\mathcal{L}_{f}$ slope characteristics in more detail. These measurements correspond closely with the expected values according to eq. 4.11, and could therefore be applied to eq. 4.4 when it is to be used for DoA estimation. 


\section{Non-linearity}

With the steering angles from $\$ 4.2 .3$, eq. 4.4 can be justified for DoA estimation. However, this approximation is not valid in general. Ignoring the spatial aliases, figure 4.8 reveals that broadening of the shaped beampattern is far from linear for larger steering angles. This is due to the non-linear relation between the array factor in $\psi$-space and $\theta$-space (viz., the sine component in the complex exponential). Although a ramp defined in the $\psi$ domain has been convenient for synthesis (\$3.2.1), it will not give a linear ramp in the $\theta$ domain. Figure 4.9 shows clearly that the resulting slope becomes concave as the MRA is steered away from $0^{\circ}$. The flattopped pattern, on the other hand, will remain flat regardless of the steering angle it is subjected to.

There are two options to enable DoA estimation for larger steering angles. Either a different slope can be defined during synthesis (one that is linear in $\theta$-space), or eq. 4.4 can be redefined to take the non-linearity into account. As the latter is less cumbersome, let $\Delta P / \Delta \theta$ represent the steepness of the slope defined by $S_{r}(\theta)$ (\$3.4.1), and let the steering angle $\theta_{d}$ be defined as the peak of the ramp. For the ramp shaped pattern chosen as the main example in this thesis, $\Delta P=-10 \mathrm{~dB}$ and $\Delta \theta=40^{\circ}$. In $\psi$-space, given a steering angle $\psi_{d}$, the power is associated with $\psi$ as follows

$$
P\left(\psi, \psi_{d}\right)=\frac{\Delta P}{\pi \sin (\Delta \theta)}\left(\psi-\psi_{d}\right)
$$

In $\theta$-space, this relation can be written as

TABLE 4.1 - Linearly approximated $\mathcal{L}_{r}$ slope characterization $\left(0.5^{\circ}\right.$ accurate $)$

\begin{tabular}{ccc} 
Steering angle & {$\left[\tilde{\theta}_{0}, \tilde{\theta}_{1}\right]$} & $P_{f}-P_{r}$ at $\tilde{\theta}_{1}$ \\
\hline $\mathcal{L}_{r}$ synthesized with $40^{\circ}$ beamwidth \\
\hline $0^{\circ}$ & {$\left[-15.0^{\circ}, 13.5^{\circ}\right]$} & $-7.5 \mathrm{~dB}$ \\
$15^{\circ}$ & {$\left[0.0^{\circ}, 29.0^{\circ}\right]$} & $-7.5 \mathrm{~dB}$ \\
$35^{\circ}$ & {$\left[17.5^{\circ}, 51.5^{\circ}\right]$} & $-7.5 \mathrm{~dB}$ \\
\hline \multicolumn{4}{c}{$\mathcal{L}_{r}$ synthesized with $20^{\circ}$ beamwidth } \\
\hline \multicolumn{4}{c}{$0^{\circ}$} & {$\left[-7.5^{\circ}, 6.0^{\circ}\right]$} & $-6.5 \mathrm{~dB}$ \\
$7.5^{\circ}$ & {$\left[0.0^{\circ}, 13.5^{\circ}\right]$} & $-6.5 \mathrm{~dB}$ \\
$15.0^{\circ}$ & {$\left[7.5^{\circ}, 21.5^{\circ}\right]$} & $-6.5 \mathrm{~dB}$ \\
$22.5^{\circ}$ & {$\left[14.5^{\circ}, 29.0^{\circ}\right]$} & $-6.5 \mathrm{~dB}$ \\
$30.0^{\circ}$ & {$\left[21.5^{\circ}, 37.0^{\circ}\right]$} & $-6.5 \mathrm{~dB}$ \\
$37.5^{\circ}$ & {$\left[28.5^{\circ}, 45.5^{\circ}\right]$} & $-6.5 \mathrm{~dB}$ \\
$44.0^{\circ}$ & {$\left[34.5^{\circ}, 53.0^{\circ}\right]$} & $-6.5 \mathrm{~dB}$ \\
\hline \multicolumn{4}{c}{}
\end{tabular}




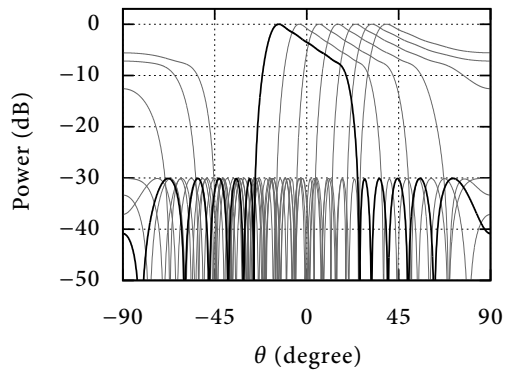

Figure 4.8 - Linearity when steered

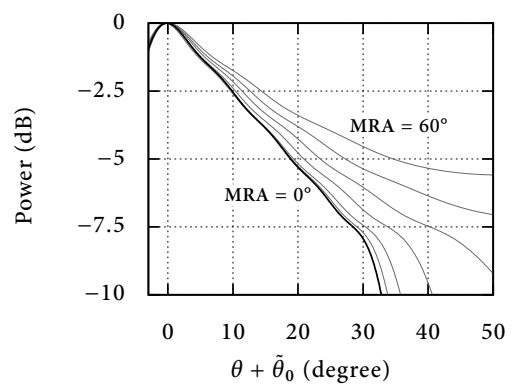

FIgURE 4.9 - Ramp slope in detail

$$
P\left(\theta, \theta_{d}\right)=\frac{\Delta P}{\pi \sin (\Delta \theta)}\left(\pi \sin (\theta)-\pi \sin \left(\theta_{d}\right)\right)
$$

according to eq. 3.7. To derive the angle of incidence from a certain power level, this expression is rewritten as follows

$$
\begin{gathered}
\frac{P\left(\theta, \theta_{d}\right) \pi \sin (\Delta \theta)}{\Delta P}=\left(\pi \sin (\theta)-\pi \sin \left(\theta_{d}\right)\right) \\
\frac{P\left(\theta, \theta_{d}\right) \sin (\Delta \theta)}{\Delta P}+\sin \left(\theta_{d}\right)=\sin (\theta) \\
\sin ^{-1}\left(\frac{P\left(\theta, \theta_{d}\right) \sin (\Delta \theta)}{\Delta P}+\sin \left(\theta_{d}\right)\right)=\theta
\end{gathered}
$$

Of course when the ramp shape is reversed, the expression becomes

$$
\sin ^{-1}\left(\frac{-P\left(\theta, \theta_{d}\right) \sin (\Delta \theta)}{\Delta P}+\sin \left(\theta_{d}\right)\right)=\theta
$$

By substituting eq. 4.4 with eq. 4.16 (or eq. 4.17 in case of a reversed ramp), the steering angle will not be of influence on the accuracy of DoA estimation.

\subsubsection{RIPPLE}

Because of various implementation aspects, it could be necessary to allow a larger ripple (chapter 5). Regardless of the specific reason, when the ripple increases, a larger error will be made in estimating the DoA. The magnitude of the error depends on the steepness of the $\Delta P / \Delta \theta$ slope chosen for $\mathcal{L}_{r}$. In order to have a convenient means of quantifying this error, the ripple will be approximated by a sinusoid. In that case, the RMS value of its amplitude can be used as the uncertainty (i.e., standard deviation) in the measured power. Given that the slope steepness depends 




FIGURE 4.10 - DoA estimation error due to ripple

on the steering angle of the pattern (as described above), a tangent should be taken to characterize the steepness for a certain power level. However, for convenience a fixed slope steepness of $-10 \mathrm{~dB} / 40^{\circ}$ will be utilized for the simulated $\pm 45^{\circ}$ elevation range.

The power measure used for DoA estimation is based on two patterns, for which it is assumed that both feature the same amount of uncorrelated ripple. This means that the ripple needs to be taken into account twice for a conservative measure of the DoA estimation accuracy. Furthermore, a ripple was defined as twice the amplitude in chapter 3 (figure 3.18). Its RMS value is thus given by ripple $/ 2 \sqrt{2}$. On the other hand, with such a definition it must be considered that deviations in the measured power can be positive or negative. As this can be expressed by twice their absolute value, a rough approximation of the error can be found with

$$
\begin{aligned}
\text { error } & =\left|\frac{\Delta \theta}{\Delta P}\right| 2 \sqrt{(\text { ripple } / 2 \sqrt{2})^{2}+(\text { ripple } / 2 \sqrt{2})^{2}} \\
& =\left|\frac{\Delta \theta}{\Delta P}\right| 2 \sqrt{\text { ripple }^{2} / 4} \\
& =\left|\frac{\Delta \theta}{\Delta P}\right| \text { ripple }
\end{aligned}
$$

This relationship between ripple and DoA estimation error is shown in figure 4.10.

\subsubsection{Estimation ACCURACY}

For increasing amounts of ripple, table 4.2 shows the expected (calculated) error based on eq. 4.18 compared to the error measured during simulations, using eq. 4.16. 
TABLE 4.2 - DoA estimation errors based on ripple

\begin{tabular}{ccccccc} 
Pattern ripple & $0.1 \mathrm{~dB}$ & $0.2 \mathrm{~dB}$ & $0.5 \mathrm{~dB}$ & $1 \mathrm{~dB}$ & $1.5 \mathrm{~dB}$ & $2 \mathrm{~dB}$ \\
\hline Calculated error & $0.4^{\circ}$ & $0.8^{\circ}$ & $2.0^{\circ}$ & $4.0^{\circ}$ & $6.0^{\circ}$ & $8.0^{\circ}$ \\
Simulated error & $1.6^{\circ}$ & $1.7^{\circ}$ & $1.7^{\circ}$ & $2.6^{\circ}$ & $3.7^{\circ}$ & $4.8^{\circ}$
\end{tabular}

The errors found by simulation generally follow a trend similar to the one predicted by eq. 4.18. Aside from the smaller values, for which it appears that another cause (e.g., signal-to-noise ratio (SNR)) determines accuracy, the estimation error is roughly linear with the ripple. For ripples larger than $0.5 \mathrm{~dB}$, the measured error is considerably smaller than the calculated one. This can be explained by the fact that the ripples of $\mathcal{L}_{f}$ and $\mathcal{L}_{r}$ are not entirely uncorrelated, in which case twice the RMS value is an exaggeration of the power uncertainty. In the next chapter it is shown that larger ripples are generally easier to realize. Having observed the trend in the simulated errors, a $0.5 \mathrm{~dB}$ ripple could therefore be an interesting compromise.

Based on a $0.1 \mathrm{~dB}$ ripple, and the non-linear characteristics of the slope properly taken into account, simulated DoA estimations are observed to be accurate up to $1.6^{\circ} \mathrm{RMS}$ with peak deviations reaching $3.5^{\circ}$. These results are based on the conditions described in $\$ 4.2 .4$, with power averaged over $48 \mu$ s and $\Upsilon=2$. This accuracy is also correlated with the sample size and the SNR. A proper statistical power analysis should be performed to determine the appropriate sample size $K$. Although such an analysis is not part of this thesis, figure 4.11 can give a rough indication of the RMS error made under different levels of SNR with various smaller sample sizes $(K=\{100,25,20,15,10,5\})$. Based on the $14 \mathrm{~dB}$ SNR that has been assumed necessary for communication, it appears that a sample size of at least 100 is needed to stay within $3^{\circ}$ RMS error.

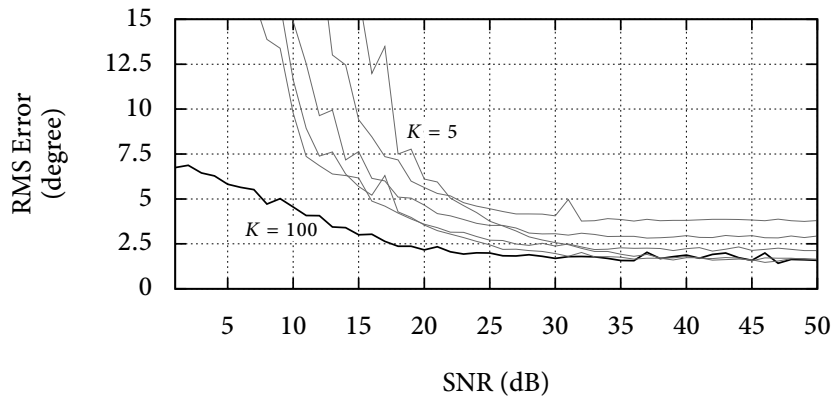

Figure 4.11 - DoA estimation with different sample sizes $K$ 


\subsection{Discussion AND FUTURE WORK}

Servicing multiple user from a single array could be approached with a TDMA solution. Although it is also possible to think of ways to control multiple beams operating in different frequency bands, this strategy does not scale well for analog switched beamforming (\$2.2). If TDMA is chosen, it must be considered that the scan angle will change between servicing different mobile devices. In addition, one may want to reduce the beamwidth (figure 4.7) for better spatial resolution. The beampattern will thus have to be updated more frequently, which can be an argument in favor of using switched delay lines (\$1.1.3) over continuous tunability.

The simulations presented in this chapter show that tracking and DoA estimation based on shaped beampatterns is possible. However, a number of assumptions had to be made. One of the assumptions is that there exists a clear LoS. With indoor scenarios, it is not entirely realistic to assume that a clear LoS is always available. The blockage of $60 \mathrm{GHz}$ signals in a non-LoS situation must be solved. However, this is an extensive topic $[20,53,89]$ that is beyond the scope of this thesis. Likewise, it may need to be investigated how beamforming on both the transmitting and receiving side can be supported. Finally, it will also be worthwhile to check compatibility with the proposed IEEE standards for $60 \mathrm{GHz}$ wireless communication $[67,68]$.

\subsection{Conclusions}

Because an all-analog (single output) beamformer does not provide the means for conventional DoA estimation, a new tracking algorithm has been developed exploiting a ramp shaped beampattern and a flat-topped beampattern. The observed relative power difference, measured whilst alternating between these two patterns, can be used either directly as a direction for beam steering, or it can be converted to the DoA by relying on the unique relationship between power and angle, featured in the ramp shaped beampattern. The use of a flat-topped pattern eliminates any unwanted dependencies on e.g. path loss.

Based on simulation results, the maximum ripple under which beam alignment can be maintained was found to be $1.5 \mathrm{~dB}$. Actual DoA estimation is slightly more sensitive to the ripple. For that purpose, a ripple no larger than $0.5 \mathrm{~dB}$ is desired. Using a 16-element antenna array, the proposed pair of shaped beampatterns can cover a $120^{\circ}$ sector. Within that angular region, the position of a mobile wireless device can then be tracked fairly accurate with an RMS error of $1.7^{\circ}$. 



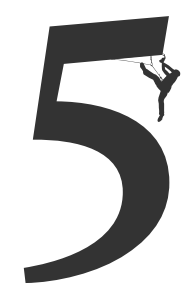

\title{
REALIZATION CONSIDERATIONS
}

\begin{abstract}
In this chapter, the implementation aspects of using shaped beampatterns for tracking purposes are considered First, the gain of the individual array elements (abstracted in the array factor) is reintroduced into the shape of the beampattern. It turns out that this can affect the shape to such an extent that the pattern is no longer suitable for tracking purposes. The Orchard-Elliott synthesis method is therefore extended such that non-isotropic gain of realistic antenna elements is compensated in the excitation values. This is achieved analytically so that the low ripple requirement can still be satisfied. Subsequently, it is demonstrated that the use of shaped patterns is feasible from a physical realization point of view. The implications for an amplitude tapered time delay beamformer will be discussed.
\end{abstract}

$\mathrm{A}$ NALYTICAL ways of pattern synthesis are often criticized for their lack of ability to take certain EM phenomena and implementation specific constraints into account. Two prominent EM effects that designers like to compensate when a beampattern is shaped are the non-isotropy of realistic array elements, known as the element factor, and the coupling between array elements $[51,96,126]$. In general, these effects are indeed difficult to capture in a closed form expression. Nevertheless, considerations for mutual coupling have been covered in literature [90, 99]. The element factor is typically neglected in the Orchard-Elliott synthesis method. Its influence on the shape of the beampattern is, however, too severe to be neglected for shaped-pattern tracking, The first part of this chapter explains how to incorporate the element factor analytically, when the array conditions from chapter 3 apply. Disadvantages with regard to the inability of specifying constraints other that those expressed in terms of the power pattern pertain mostly to the maximum amplitude ratio of the complex excitation values. From an implementation perspective, very large ratios are difficult to realize. Minimization of this ratio is therefore desired.

Parts of this chapter have been published in [TB:6]. 
Although it is true that the Schelkunoff form does not allow the excitations to be controlled directly, it possesses other properties that can be exploited to optimize the excitation values for implementation specific purposes. Those properties, as well as other physical realization aspects will be dealt with in the second part of this chapter.

\subsection{ELEMENT FACTOR COMPENSATION}

Most of the advocated solutions for synthesis problems where the EM imperfections matter involve usage of optimization techniques. As discussed in chapter 3 , these are sufficiently generic that one could, in theory, perform a full wave simulation of the beampattern between each optimization step. Thus, practically any parasitic effect can be included in calculating the shape of the pattern. However, these techniques were found to be very difficult to use in obtaining the type of pattern needed for tracking as explained in chapter 4. With Orchard-Elliott synthesis, it is commonly accepted that the shape of the actual beampattern will deviate slightly from the synthesized shape. In certain application domains, such as a ground mapping radar [41], this difference can indeed be tolerated. This is not the case for shaped-pattern tracking. Consider for example the influence of a realistic (measured) element factor on the ramp shaped beampattern. In figure 5.1, the shaped portion of the power pattern conjoins with the element factor's boresight (viz., where its gain is usually optimal). The effect of neglecting the element factor is therefore marginal; it is mostly noticeable in the less important sidelobe region. When the shaped beam is positioned closer to endfire, as depicted in figure 5.2, the effect of non-isotropic gain will become more severe. It will be clear that such shape differences may cause problems for the tracking algorith ${ }^{1}$. The decaying gain of such an element factor must be taken into account by the synthesis algorithm, such that the array excitations compensate it.

In [10] it is suggested that Orchard-Elliot synthesis can be applied to non-isotropic antenna elements, however without further elaboration. This section details exactly how to incorporate the (measured) directivity of physical antennas. In principle it requires only a minor modification, however, practical aspects make the procedure non-trivial. For convenience, the most important assumptions and steps taken in Orchard-Elliott synthesis shall be recapitulated first.

\subsubsection{ANALYSIS}

Thus far, the power of the array factor was considered to be equivalent to the actual beampattern. In a mathematical sense, if the antenna elements are identical and oriented in the same direction [123], the beampattern $B(\theta)$ of an equispaced linear

\footnotetext{
${ }^{1}$ Note that this extreme case will not occur in the proposed tracking mechanism from chapter 4, because the ramp shape is reversed when the steering angle is negative. Nevertheless, for this section it is a good example to illustrate various details.
} 




FIGURE 5.1 - Element factor influence on a ramp shaped beampattern at $\theta_{d}=15^{\circ}$

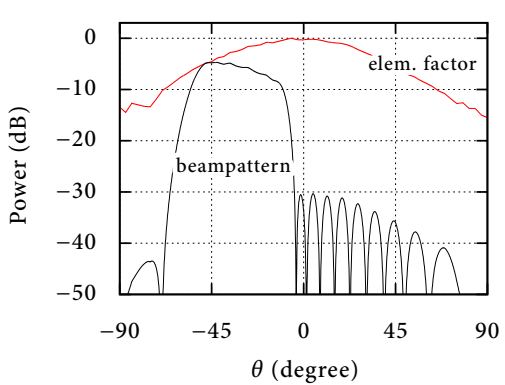

FIGURE 5.2 - Element factor influence on a ramp shaped beampattern at $\theta_{d}=-30^{\circ}$

antenna array is a multiplication of the array factor $F(\theta)$ and a common element factor $E(\theta)$

$$
B(\theta)=F(\theta) E(\theta)
$$

Essentially the element factor $E(\theta)$ was simplified to the value 1 . The array factor itself was given by

$$
F(\theta)=\sum_{n=0}^{N-1} I_{n} e^{j n \frac{2 \pi d}{\lambda} \sin (\theta)}
$$

as discussed in chapter 3 . Recall that a spacing of $d=1 / 2 \lambda$ results in a visible region [123] of $180^{\circ}$. This $180^{\circ}$ periodicity will be important in this section. With the substitution of $\psi=k d \sin (\theta), w_{n}=e^{j \psi}$ and $w_{n}=e^{a_{n}+b_{n}}$, eq. 5.2 was rewritten to

$$
\begin{aligned}
G(\psi) & =\sum_{n=1}^{N-2} 10 \log _{10}\left[1-2 e^{a_{n}} \cos \left(\psi-b_{n}\right)+e^{2 a_{n}}\right] \\
& +10 \log _{10}[2(1+\cos (\psi))]+C_{1}
\end{aligned}
$$

in order to control its shape based on (decomposed) polynomial roots. Starting from a suitable initial pattern, the synthesis procedure iteratively perturbed $a_{n}$ and $b_{n}$ simultaneously to find root positions that cause $G(\psi)$ to approximate a specified shape $S(\psi)$ within specified limits. For the proposed element factor compensation, the relevant steps of one such iteration are finding the local maxima of $G(\psi)$ in the sidelobe region (II) and the minima and maxima of the ripple created by $G(\psi)-S(\psi)$ in the shaped region (I). Following the classical Orchard-Elliott approach, these values are derived from eq. 5.3. Now the objective is to obtain them from a function $G E(\psi)$, which incorporates the element factor $E(\psi)$. 


\subsubsection{INCORPORATING THE ELEMENT FACTOR}

As before, almost any pattern can serve as a starting point as long as it leads to well defined minima and maxima for the shaped region $\left(a_{n} \neq 0\right)$. However, fixation of the main lobe adjacent to $-\pi$ now becomes a requirement. The pattern proposed in the original work of Orchard et al. [91] (\$3.3) shall be assumed. Its shape is illustrated by the solid line in figure 5.3 .

Recall from chapter 3 that, in order to specify the shape for region I between two arbitrarily chosen angles $\psi_{0}$ and $\psi_{1}\left(\psi_{1}>\psi_{0}\right)$, the shape function $S(\psi)$ was given an offset $\delta \psi$

$$
S^{\prime}(\psi)=S(\psi-\delta \psi)
$$

so that the shape can still be defined relative to the main lobe's peak, denoted by $\psi_{m}$. Once the pattern had converged on $S^{\prime}(\psi)$, all root positions found by synthesis $\left(b_{n}\right)$ were rotated $-\delta \psi$ around the unit circle simultaneously $\left(b_{n}=\stackrel{\circ}{b}_{n}-\delta \psi\right)$ to shift the shaped region back to the originally specified range $\left[\psi_{0}, \psi_{1}\right]$. When the element factor is uniform over $\psi$, this process will not affect the shape of the pattern, as shown by the dotted line in figure 5.3.



FIGURE 5.3 - Beampattern (shifted) using isotropic antennas 


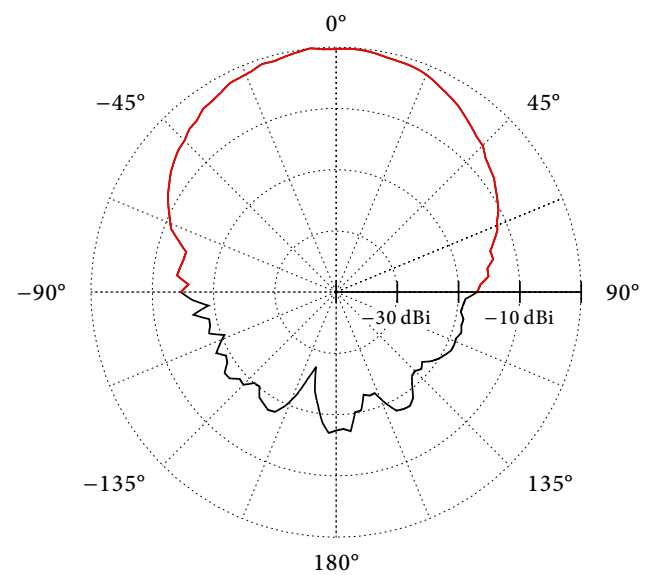

FIGURE 5.4 - Antenna gain, measured every $3^{\circ}[29]$

\section{Closed form element factor}

Incorporating the element factor in eq. 5.3 requires that it is characterized first. Most likely, this characterization will be a set of measurements rather than a closed form expression. Let those measurements be denoted by e. To serve as an example element factor, the gain of a $10 \mathrm{GHz}$ wideband antenna was measured every $3^{\circ}$ in the far field. This type of antenna is currently deployed in an experimental optical beam steering setup [29], which is a simplified version of the infrastructure described in chapter 1 . Figure 5.4 shows the measured gain in polar coordinates. Given the array's $180^{\circ}$ visible region at $d=1 / 2 \lambda$, only the part between $-90^{\circ}$ and $90^{\circ}$ around boresight $\left(0^{\circ}\right)$ will be relevant. Note also that the gain is measured in $\theta$-space, which means that a conversion to $\psi$-space is needed $(\$ 3.1)$.

As defined above, the element factor in decibels can be included in eq. 5.3 with

$$
G E(\psi)=G(\psi)+E(\psi)
$$

However, a closed form expression $E(\psi)$ for the element factor is not available, only a set of measurements e. One could evaluate $G(\psi)$ at the same angles as e to obtain a numerical representation of eq. 5.5, but there are some downsides to this approach. Firstly, synthesis relies on finding local extrema which requires a certain 'smoothness' that a numerical representation does not exhibit. Secondly, when a very small ripple is desired (e.g., $0.1 \mathrm{~dB}$ ), the minima and maxima eventually become so small that they are difficult to detect using numerical methods. In most cases a polynomial fit can be applied to $\mathbf{e}$ in order to obtain the expression $E(\psi)$. The extrema can then be found algebraically, which is more robust. 


\section{Element factor alignment}

The result of including the element factor can be seen in figure 5.5. Unlike the array factor $G(\psi)$, the beampattern's shape $G E(\psi)$ is clearly affected by a shifting operation. To obtain excitations that compensate the element factor correctly, the $\psi_{m}$ peak must coincide with $\psi_{0}$ at all times during synthesis. Such an alignment can be achieved by shifting either $E(\psi)$ along with $S(\psi)$, or by shifting $G(\psi)$ such that $\psi_{m}=\psi_{0}$ always holds. If one of the polynomial's roots is fixed at $-\pi$, as suggested earlier, $G(\psi)$ itself is also fixed. For this reason, and the fact that the latter alternative was found to be cumbersome, alignment of $E(\psi)$ will be explained.

Because $G(\psi)$ is $2 \pi$ periodic like $F(\theta)$ is $180^{\circ}$ periodic, it repeats itself outside the visible region [123]. Effectively this causes $G(\psi)$ to wrap around $-\pi / \pi$ when shifted. Due to this property and because only the visible region is of interest, for synthesis purposes $E(\psi)$ may be wrapped around the visible region in the direction of $\delta \psi$

$$
E^{\prime}(\psi)=E(\psi-\delta \psi+\pi) \bmod 2 \pi
$$

See figure 5.6 for the effect. Substituting $E^{\prime}(\psi)$ for $E(\psi)$ in eq. 5.5 brings forth an expression in which the element factor and array factor are correctly aligned. It should be noted that $\psi_{m}$ may change slightly after displacing the roots of $G(\psi)$. This means that $\delta \psi$ needs to be recalculated and $E(\psi)$ realigned for each synthesis iteration. It is also important to realize that the sign of $\delta \psi$ changes when $\psi_{0}<\psi_{m}$.

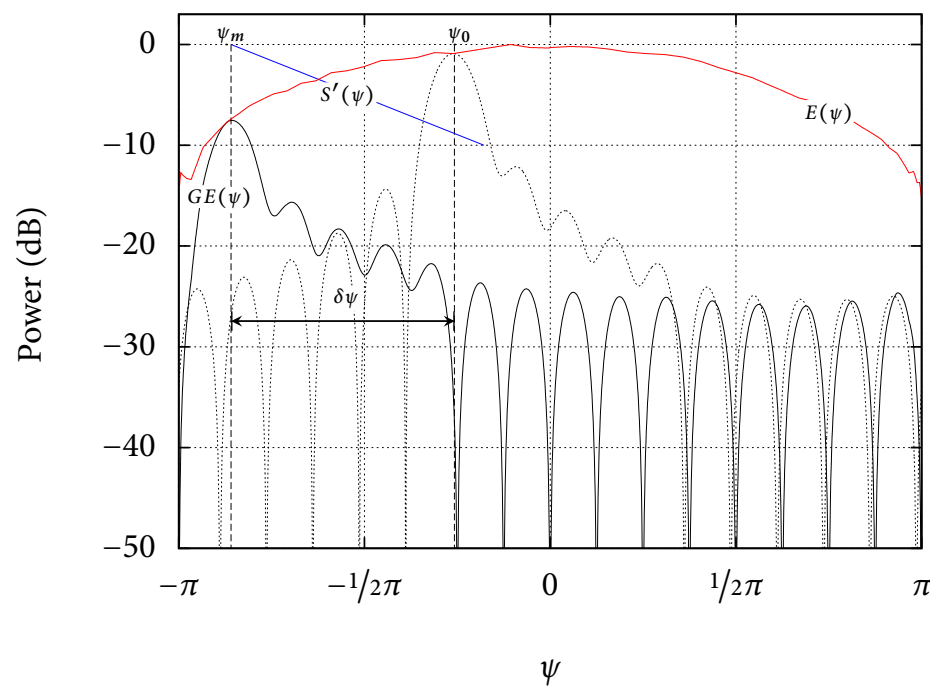

FIGURE 5.5 - Beampattern (shifted) using non-isotropic antennas 


\section{Piecewise approximation}

The shifted version $\mathbf{e}^{\prime}$ of the measured antenna gain e can often not be approximated well by a polynomial, because wrapping the element factor introduces a discontinuity (figure 5.6), or at least a sudden jump, at $\psi_{p}$

$$
\psi_{p}=\left\{\begin{array}{rr}
\pi+\delta \psi, & \delta \psi<0 \\
-\pi-\delta \psi, & \delta \psi>0
\end{array}\right.
$$

To what extent the approximation suffers from this discontinuity can be seen well in figure $5.7(\mathrm{a})$. Much better results are obtained when $E^{\prime}(\psi)$ is fitted piecewise from $-\pi$ to $\psi_{p}$, and from $\psi_{p}$ to $\pi$. This type of approximation is illustrated in figure 5.7(b). Let $E_{l}(\psi)$ be the curve that is fitted to the data points from $\mathbf{e}^{\prime}$, where $\psi \leq \psi_{p}$, and $E_{r}(\psi)$ the curve that is fitted to the data from $\psi>\psi_{p}$. The expression for the beampattern, including the aligned element factor, then becomes

$$
G E(\psi)= \begin{cases}G(\psi)+E_{l}(\psi), & \psi \leq \psi_{m} \\ G(\psi)+E_{r}(\psi), & \psi>\psi_{m}\end{cases}
$$

Using eq. 5.8 for synthesis will yield a beampattern which does not suffer from the non-uniform gain of the physical antennas used in the array.

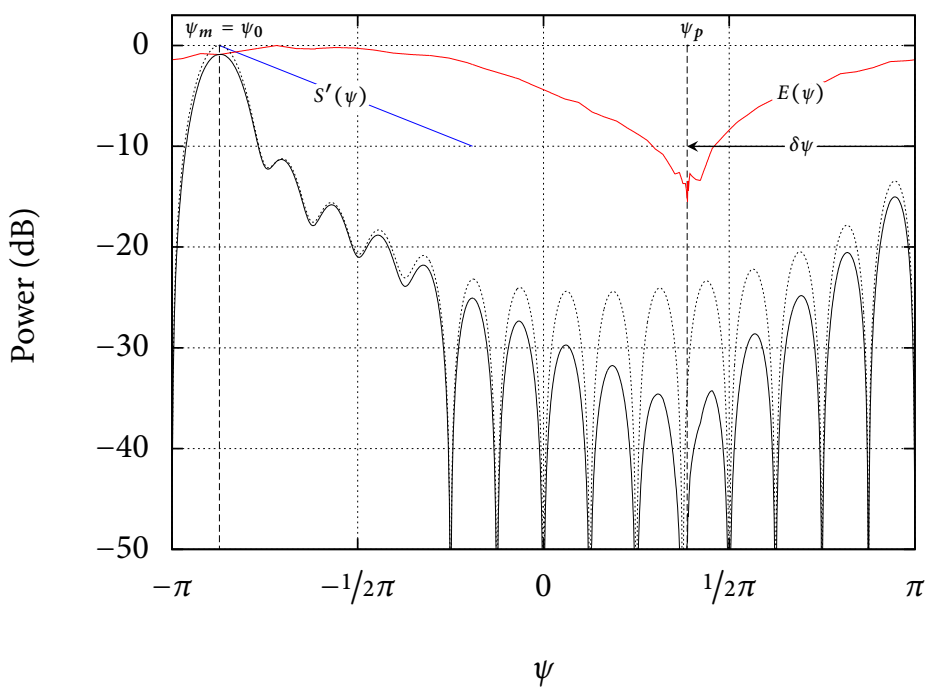

FIGURE 5.6 - Element factor alignment 
(a) $26^{\text {th }}$ order curve fitting of $\mathbf{e}^{\prime}$

80

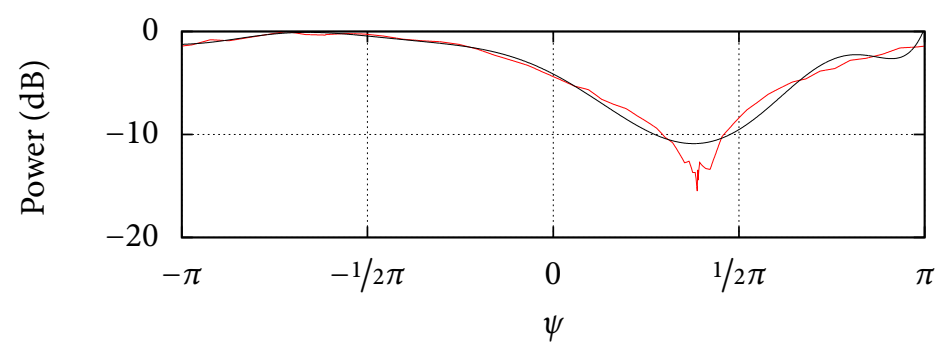

(b) $5^{\text {th }}$ order piecewise curve fitting of $\mathrm{e}^{\prime}$

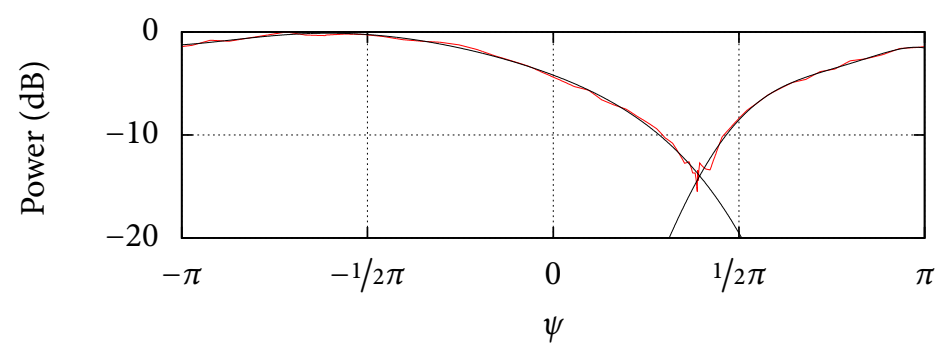

Figure 5.7 - Shifted element factor approximation.

\subsubsection{Application to A RAMP SHAPED PATtern}

To demonstrate the effectiveness of the proposed modifications, the familiar ramp shaped pattern has again been synthesized with a maximum ripple of $0.1 \mathrm{~dB}$, now taking the measured gain of the $10 \mathrm{GHz}$ antenna (figure 5.4) into account. The desired shape (figure 5.8) is defined as

$$
S_{r}(\theta)=\left\{\begin{array}{lr}
-30, & \theta<-45^{\circ} \\
-1 / 4(\theta+45)-5, & -45^{\circ} \leq \theta \leq-5^{\circ} \\
-30, & \theta>-5^{\circ}
\end{array}\right.
$$

Region I ( $\left.\left[\theta_{0}, \theta_{1}\right]\right)$ is intentionally chosen close to endfire, where the non-uniform gain of a realistic antenna pattern is most notable. The beampattern resulting from standard Orchard Elliott synthesis is plotted as the dotted line. Note that the power is almost $5 \mathrm{~dB}$ below the specification at $\theta=-45^{\circ}$. Such a difference would lead to 


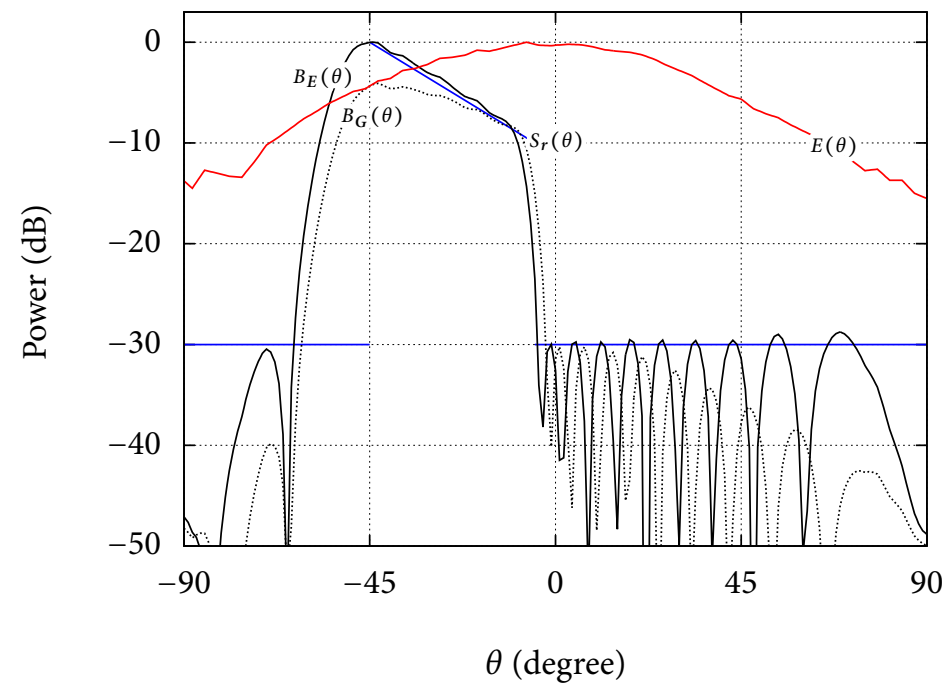

FIGURE 5.8 - Shaped pattern synthesis with element factor compensation $\left(B_{E}\right)$ and without compensation $\left(B_{G}\right)$

an unacceptably large error in the tracking and DoA estimation method explained in chapter 4. Using the proposed modifications, the pattern's shape is much closer to specification, as illustrated by the solid line. With an element factor as used here, the algorithm is still capable of reducing the ripple to $0.1 \mathrm{~dB}$. Note that in figure 5.8, the small deviations observable for the sidelobe heights are caused by the polynomial approximation near the discontinuity.

\subsubsection{Computational Stability}

Some problems may be experienced from the element factor extension. Finding the local maxima in region II becomes problematic when $\psi_{p}$ coincides with one of the sidelobes. The sudden jump of $E(\psi)$ at angle $\psi_{p}$ then causes the appearance of two small peaks in $G E(\psi)$, as depicted in figure 5.9. This is a problem because the algorithm expects exactly $N_{2}$ maxima while $N_{2}+1$ maxima are found. To solve this issue, let $w_{l}$ be the first root to the left of $\psi_{p}$ and $w_{r}$ the first root to the right of $\psi_{p}$. One could interpolate the expected $G E(\psi)$ curve between $w_{l}$ and $w_{r}$. However, this requires a fair amount of computation while for most applications a satisfactory single peak location, say $\psi_{r}$, can be found using $G(\psi)$ on that interval. Depending on whether $\psi_{r} \leq \psi_{p}$ or $\psi_{r}>\psi_{p}, G\left(\psi_{r}\right)+E_{l}\left(\psi_{r}\right)$ or $G\left(\psi_{r}\right)+E_{r}\left(\psi_{r}\right)$ will give the desired height of the sidelobe. Because a similar repair would be extremely hard for region I, shaping patterns where $\psi_{0}<\psi_{p}<\psi_{1}$ (i.e., defined across the visible region) are best avoided. 


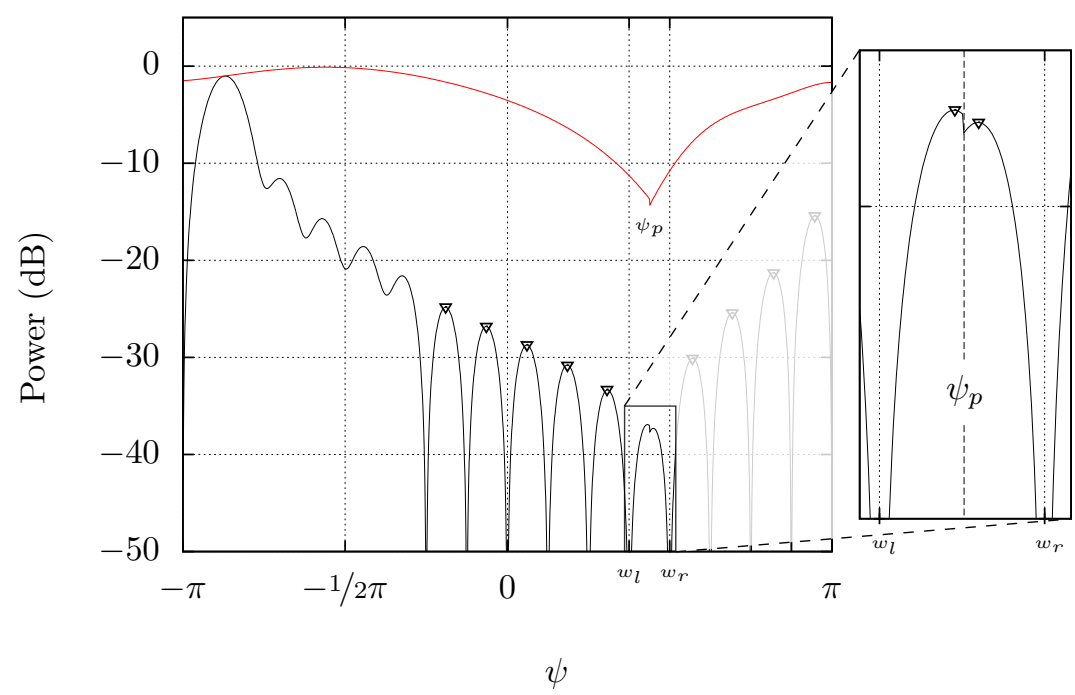

FIGURE 5.9 - Problems with peak detection due to element factor alignment

A different problem may arise when $\psi_{0}$ and $\psi_{m}$ are close together. When $\psi_{p}$ ends up too close to $-\pi$ or $\pi$, there will be insufficient data points to fit $E_{l}(\psi)$ or $E_{r}(\psi)$ uniquely. When this is the case, $G E(\psi)$ should be replaced by either $G(\psi)+E_{l}(\psi)$ or $G(\psi)+E_{r}(\psi)$, for all $\psi$. At which point this is needed depends on how fine grained $E(\theta)$ was measured. As a rule of thumb it can be said that when $\psi_{p}<\psi_{m}$, it is better to use $G(\psi)+E_{r}(\psi)$ instead of eq. 5.7, and $G(\psi)+E_{l}(\psi)$ when $\psi_{m}$ is to the right of the right-most peak in $G(\psi)$.

\subsubsection{Discussion}

There are downsides to element factor aware synthesis. Firstly, one can expect longer computation times. These are mainly the result of additional calculations required to arrive at $G E(\psi)$. However, the convergence rate now also varies with the positioning of region I. With a halting condition based solely on the ripple (\$3.3), table 5.1 provides an overview of the convergence rates for synthesizing ramp- (not reversed), and flat-topped patterns, with a maximum allowed ripple of $0.1 \mathrm{~dB}$. In addition, the $\csc ^{2}(\theta) \cos (\theta)$ example from [91] has been included for comparison. The second column represents Orchard-Elliott synthesis (based on $G$ ) while columns three to seven list element factor synthesis (based on GE) for different locations of region I. As can be seen, element factor compensation mostly results in slower convergence. However, there are some occasions in which the element factor is beneficial for the converge rate. The additional computation time per itera- 
TABLE 5.1 - Synthesis convergence (\# iterations)

\begin{tabular}{lcccccc} 
& $G$ & $\begin{array}{c}G E \\
{\left[-60^{\circ},-20^{\circ}\right]}\end{array}$ & $\begin{array}{c}G E \\
{\left[-40^{\circ}, 0^{\circ}\right]}\end{array}$ & $\begin{array}{c}G E \\
{\left[-20^{\circ}, 20^{\circ}\right]}\end{array}$ & $\begin{array}{c}G E \\
{\left[0^{\circ}, 40^{\circ}\right]}\end{array}$ & $\begin{array}{c}G E \\
{\left[20^{\circ}, 60^{\circ}\right]}\end{array}$ \\
\hline flat & 5 & 6 & 6 & 6 & 6 & 6 \\
ramp & 6 & 12 & 11 & 13 & 22 & 14 \\
$\csc ^{2}$ & 13 & 6 & 11 & 13 & 14 & 14
\end{tabular}

tion varies between $40 \%$ and $45 \%$. It should also be noted that synthesizing shaped patterns for a specific element factor means that a pattern needs to be resynthesized when the location of region I is changed. Lastly, an increased complexity in the excitations $I_{n}$ is likely, as already pointed out in [10]. One should in particular be aware of a higher amplitude range (\$5.3), which emphasizes the mutual coupling effect as discussed in the next section.

\subsection{Mutual COUPLing CONSIDERATIONS}

In the presence of severe mutual coupling [86], there are several different actions that may lead to improvement:

»Choosing a larger element spacing $d$

» Refining the antenna element design

» Reducing the amplitude ratio between the complex excitation values

»Employing coupling-aware synthesis techniques

The first option is the least likely to be chosen due to the undesired side-effect on the array's visible region (\$3.1.1). However, when the other options offer no solution, increasing the distance between antennas generally results in less coupling [4]. A well designed antenna element will also help reduce the EM interaction, but that subject is outside the scope of this thesis. The last two options are most fitting for the work presented here. Reduction of the amplitude ratio is discussed in the next section, a brief overview of relevant synthesis techniques capable of taking into account the influences of mutual coupling is given here.

In literature, two examples $[90,99]$ can be found where the essence of OrchardElliott synthesis is used in conjunction with mutual coupling considerations. The word 'essence' is used because the necessary modifications prevent the array factor from being transformable to the Schelkunoff representation. In both references, the array excitations are modeled as independent voltage sources that are modified by an impedance/cross-coupling matrix. The latter is obtained either by using comprehensive EM simulation software or by measurements. As the additional matrix multiplications make the $I_{n}$ parameters in eq. 5.2 dependent on each other, factorization of the polynomial is no longer valid. A transformation from $w_{n}$ to $I_{n}$ is still possible, either by Vieta’s theorem [99] or as explained in \$3.1.1. Thus, in 
principle $a_{n}$ and $b_{n}$ from Orchard-Elliott synthesis can still be used as the main parameters to be adjusted. However, due to the lack of a differentiable relation between the variables and the pattern shape, all $\mathbf{g}$ and $\psi$ values (i.e., ripple $\mathrm{min} / \mathrm{max}$ and sidelobe peaks) need to be found numerically. In [9o], a genetic algorithm is used to minimize the error in a least squares sense. The authors of [99] proceed as in the original work by Orchard et al., where the main difference lies in the fact that all partial derivatives for the Jacobian matrix $\mathbf{A}(\$ 3.3)$ must be evaluated numerically. It is interesting to note that in both approaches, numerical inclusion of the element factor is also allowed. In [99] it was found that the element factor has more influence on the pattern shape than mutual coupling, based on a $1 / 2 \lambda$ cross-coupling matrix obtained by full wave EM simulations.

Subjected to the effects of mutual coupling, the resulting pattern ripple shown in [90] and [99] is fairly large (between 1 to $2 \mathrm{~dB}$ ). Because it is not known whether this ripple can be improved or not, it will be important to prevent mutual coupling as much as possible, instead of relying on the possibility to compensate its effect in the array excitations.

\subsection{EXCITATION REALIZATION}

Taking into account the need for steering, both the ramp-shaped beampattern and the flat-topped beampattern require an amplitude and phase controlled beamformer. Viz., their complex array excitations $I_{n}$ translate to an amplitude $\left(\left|I_{n}\right|\right)$ and a relative accumulated phase difference $\angle I_{n}$, as discussed in chapter 2 . Not every set of excitations is feasible for realization. In particular those with a large maximum amplitude ratio

$$
\frac{I_{\max }}{I_{\min }}=\frac{\max \left\{\left|I_{n}\right|\right\}}{\min \left\{\left|I_{n}\right|\right\}}
$$

will pose problems. Aside from the possibility of requiring unrealistic amplification values, a large amplitude range will also increase the probability of experiencing mutual coupling effects in the beampattern shape [44, 91]. Orchard-Elliott synthesis $^{2}$ features one important attribute to alleviate the requirements in terms of implementation. For the shape of a power pattern, it does not matter if the roots in region I are moved radially inward or outward from the unit circle [44]. In other words, for every root $w_{n}=e^{a_{n}+j b_{n}}$ in region I, it does not matter if the $b_{n}$ value is positive or negative. This gives the designer $2^{N_{1}}$ combinations of roots to choose from, each resulting in a different set of excitations.

\subsubsection{Amplitude}

With these different root combinations at the disposal of the designer, there are three methods of reducing the amplitude range for the $\mathcal{L}_{r}$ and $\mathcal{L}_{f}$ beampatterns:

\footnotetext{
${ }^{2}$ Or any of its derivatives discussed in this thesis.
} 


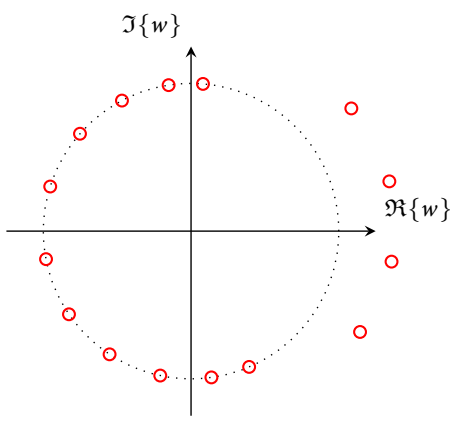

FigURE 5.10 - Default root locations

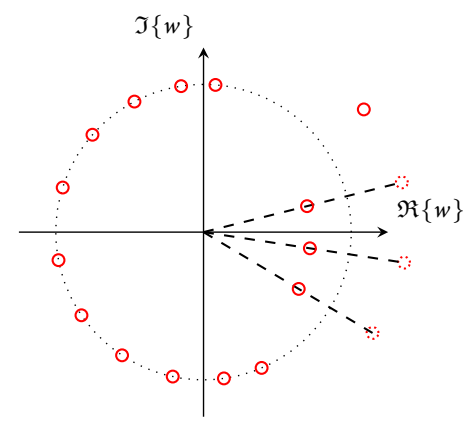

FIgURE 5.11 - Favorable root locations

" Finding the optimal combination of root locations

» Allowing a (slightly) larger ripple

»Choosing a different sidelobe topography

As changing the root locations has no effect on the shape of the pattern, it is the most elegant alternative and should be considered first.

By default, Orchard-Elliott synthesis will give $N_{1}$ roots positioned outside the unit circle, as depicted in figure 5.10. These particular root positions belong to the (broadside-steered) $\mathcal{L}_{r}$ pattern, synthesized with the element factor (from figure 5.4) compensated. The phase and amplitude distribution of the excitations resulting from these root locations have been listed in the first two columns of table 5.2. The consecutive two columns list the excitation values of the matching $\mathcal{L}_{f}$ pattern. From these distributions it can be concluded that the $\mathcal{L}_{r}$ pattern would require a maximum relative amplification of 8.52/0.66 $\simeq 12.91$ (column 1 ) while the excitations of $\mathcal{L}_{f}$ demand a factor 10.33/0.32 $\simeq 32.28$ amplification (column 3 ). Especially the latter would be hard to implement at high frequencies. When the $2^{\text {nd }}, 3^{\text {rd }}$ and $4^{\text {th }}$ root of $\mathcal{L}_{r}$ are relocated to their angular reciprocal locations, as shown in figure 5.11, a set of excitations is found that requires only $2.41 / 0.41 \simeq 5.88$ amplification (see column 5). In a similar fashion, the roots of $\mathcal{L}_{f}$ can be manipulated to arrive at the much less demanding amplitude range of 3.26/0.87 23.75 (column 7).

Finding the optimal set of excitations requires that every combination of roots is evaluated exhaustively. In these examples there are just four $N_{1}$ roots (i.e., $2^{N_{1}}=16$ combinations), so it should not lead to any complications. However, when a large number of roots is moved off the unit circle, it may help to employ a GA as proposed in [14]. Table 5.3 is the result of investigating all possible amplitude distributions for both pattern shapes, steered to the various scan angles discussed in chapter 4 . Every listed value can be realized [104]. From table 5.3 it can also be concluded that the ramp shaped pattern is generally harder to realize, and that compensation of the element factor will increase the amplitude ratio when its diminishing gain works counter productive for the desired pattern shape. This conclusion was one 
TABLE 5.2 - Excitation amplitude $\left(\left|I_{n}\right|\right)$ and phase $\left(\angle I_{n}\right)$

\begin{tabular}{rrrr|rrrrr}
\hline \multicolumn{2}{c}{$\begin{array}{c}I_{n} \rightarrow \mathcal{L}_{r} \\
\text { (default) }\end{array}$} & \multicolumn{2}{c}{$\begin{array}{c}I_{n} \rightarrow \mathcal{L}_{f} \\
\text { (default) }\end{array}$} & \multicolumn{2}{c}{$\begin{array}{c}I_{n} \rightarrow \mathcal{L}_{r} \\
\text { (favorable) }\end{array}$} & \multicolumn{2}{c}{$\begin{array}{c}I_{n} \rightarrow \mathcal{L}_{f} \\
\text { (favorable) }\end{array}$} \\
\hline 3.46 & -1.85 & 3.57 & -1.70 & 0.54 & -1.85 & 1.00 & -1.70 \\
5.94 & -1.54 & 6.99 & -1.54 & 0.41 & -0.09 & 1.00 & -0.94 \\
8.52 & -1.32 & 10.33 & -1.44 & 0.61 & 0.64 & 1.33 & -0.10 \\
7.84 & -0.95 & 9.49 & -1.29 & 1.12 & 1.39 & 2.52 & 0.80 \\
4.84 & -0.43 & 5.07 & -1.16 & 1.18 & 2.34 & 3.26 & 1.31 \\
2.99 & 0.91 & 0.79 & 1.52 & 1.45 & -2.91 & 3.04 & 1.85 \\
3.75 & 2.03 & 3.91 & 2.13 & 2.09 & -2.10 & 2.67 & 2.78 \\
3.09 & 2.65 & 3.20 & 2.27 & 2.18 & -1.40 & 3.23 & -2.83 \\
1.68 & -2.45 & 0.32 & 3.12 & 1.93 & -0.55 & 3.19 & -2.69 \\
1.91 & -0.97 & 1.97 & -0.69 & 2.21 & 0.38 & 2.35 & 3.09 \\
1.71 & -0.34 & 1.76 & -0.59 & 2.41 & 0.90 & 2.83 & 2.37 \\
0.98 & 0.74 & 0.29 & 0.18 & 2.04 & 1.01 & 3.17 & 2.02 \\
1.03 & 2.58 & 1.13 & 2.83 & 1.25 & 0.67 & 2.32 & 1.82 \\
0.66 & 3.02 & 0.71 & 2.78 & 1.38 & 0.07 & 1.30 & 1.10 \\
0.75 & -2.63 & 0.39 & -2.96 & 1.14 & -0.12 & 0.87 & 0.35 \\
1.00 & 0.00 & 1.00 & 0.00 & 1.00 & 0.00 & 1.00 & 0.00 \\
\hline
\end{tabular}

of the reasons to recommended reversing the ramp shape such that its slope always follows the element factor's decay, as stated in \$4.2.2.

TABLE 5.3 - Maximum amplitude ratios $\left(I_{\max } / I_{\min }\right)$ after repositioning of roots

\begin{tabular}{cccccc} 
& $\theta_{d}=-34^{\circ}$ & $\theta_{d}=-15^{\circ}$ & $\theta_{d}=0^{\circ}$ & $\theta_{d}=15^{\circ}$ & $\theta_{d}=34^{\circ}$ \\
\hline $\mathcal{L}_{r}$ & 6.77 & 5.00 & 5.88 & 5.30 & 3.65 \\
$\mathcal{L}_{f}$ & 5.30 & 3.92 & 3.75 & 3.98 & 5.33
\end{tabular}

Further alleviation of the amplitude requirements could be achieved by allowing an increased ripple in region I. It has been known since the introduction of OrchardElliott synthesis that diminishing the ripple increases excitation value complexity. As demonstrated in chapter 4 , increasing the ripple up to $0.5 \mathrm{~dB}$ will only have marginal effect on the tracking algorithm. The difference in amplitude ratios can be seen by comparing table 5.4 with table 5.3. It this particular example, the allowed ripple increase reduces the maximum needed amplification from 6.77 to 5.22.

TABLE 5.4 - Maximum amplitude ratios based on $0.5 \mathrm{~dB}$ ripple

\begin{tabular}{cccccc} 
& $\theta_{d}=-34^{\circ}$ & $\theta_{d}=-15^{\circ}$ & $\theta_{d}=0^{\circ}$ & $\theta_{d}=15^{\circ}$ & $\theta_{d}=34^{\circ}$ \\
\hline $\mathcal{L}_{r}$ & 5.22 & 3.62 & 4.15 & 4.54 & 3.20 \\
$\mathcal{L}_{f}$ & 4.42 & 3.39 & 2.90 & 3.27 & 5.09 \\
$\mathcal{L}_{f}$ & 4.42 & 3.39 & 2.90 & 3.27 & 5.09
\end{tabular}




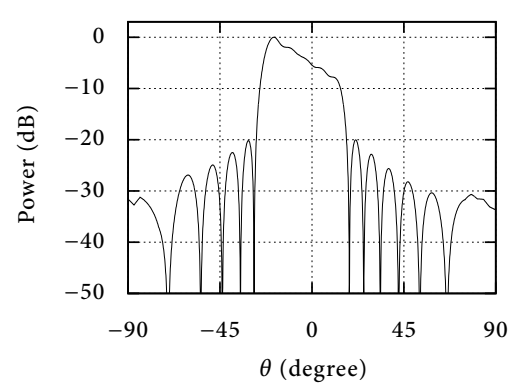

FIGURE $5.12-E(\theta)$ compensated ramp with a $0.5 \mathrm{~dB}$ ripple and Taylor sidelobes

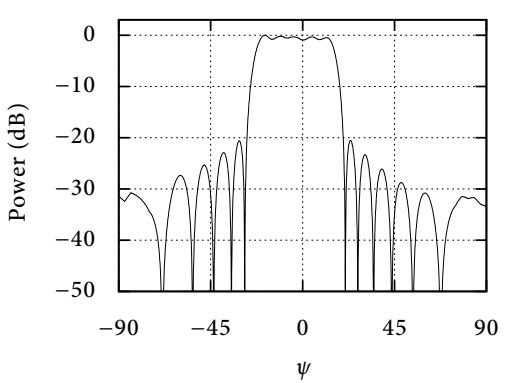

FIGURE $5.13-E(\theta)$ compensated flat-top with a $0.5 \mathrm{~dB}$ ripple and Taylor sidelobes

Lastly, one could use a less demanding sidelobe topography such as the 'Taylor'-like distribution [120] shown in figures 5.12 and 5.13. The use of Taylor sidelobes implies a further reduction of the maximum observed amplitude ratio to 4.98 . Besides a reduction of the maximum amplitude ratio, this may also help in smoothing out the progressive amplitude values (i.e., an increasing/decreasing trend). This can be of value because the presence of an upswing in the excitation amplitudes exacerbates mutual coupling effects [91].

\subsubsection{PHASE}

A study of root combinations does not identify any particular superiorities among the excitation phase distributions. The values needed to implement the proposed tracking method are not harder to manufacture than those used in other adaptive array methods [7]. However, they do not feature the regularity that a conventional beampattern exhibits. Implementation-wise, this irregularity is a drawback because integer multiples of a singular (minimum) value require less design effort.

\section{Time delays}

In the analog domain, $\angle I_{n}$ is induced either by a phase shifter or a time delay. An appropriate phase shift for the $n^{\text {th }}$ channel would directly be given in radians by $\varphi_{n}=\angle I_{n}$. The equivalent time delay $\tau_{n}$ is found by

$$
\tau_{n} \equiv \frac{\varphi_{n}}{2 \pi} \frac{\lambda}{c}
$$

where $c$ is the speed of light and $\lambda$ is determined by the center frequency $\omega_{c}$. For a proper time delay conversion, all $\varphi_{n}$ values between $-\pi$ and $\pi$ will have to be unwrapped first. Furthermore, in the presence of negative phase shifts, each delay shall also be given an appropriate offset as negative delays cannot be realized. To exemplify the conversion process, all the favorable phase components from table 5.2 
have been listed in table 5.5 as true time delays. These delays are based on a center frequency $\omega_{c}=61.5 \mathrm{GHz}$, which is exactly in the middle of the entire unlicensed $60 \mathrm{GHz}$ frequency band (figure 1.1). Although, the resulting individual time delays in table 5.5 are not particularly dissimilar from other OTTD solutions [94], they do not posses the property of progressing in discrete $\delta \tau_{n}$ steps as discussed in \$2.2. The use of shaped beampatterns is therefore reserved to beamformer structures that can impose (almost) arbitrary delays. This implies that the use of cascaded (identical) delays cannot be employed unless they are continuously tunable.

\section{Bandwidth}

One of the more often quoted advantages of (optical) true time delay beamforming is its resilience against the beam squint problem $(\$ 2.2)$. For a shaped beampattern, this argument does not hold as strongly as for a conventional beampattern. A wideband beamformer based on true time delay will ensure that the MRA of the beampattern always points to the same $\theta$ regardless of the operating frequency $\omega$ [7]. The null positions relative to the MRA will still change when the frequency deviates from $\omega_{c}$. In a conventional beampattern this typically expresses itself in the width of the main lobe. For a shaped beampattern, the filled nulls (chapter 3 ) are inherently part of the main beam so its shape will slightly change as $\omega$ diverges from $\omega_{c}$.

To evaluate the shape of the beampattern in terms of time delays and operating frequency, the array factor from eq. 5.1 is rewritten to

TABLE 5.5 - Time delay conversion $\mathcal{L}_{r}$ and $\mathcal{L}_{f}$

\begin{tabular}{|c|c|c|c|}
\hline \multicolumn{2}{|c|}{$\mathcal{L}_{r} @ \theta_{d}=0^{\circ}$} & \multicolumn{2}{|c|}{$\mathcal{L}_{f} @ \theta_{d}=0^{\circ}$} \\
\hline$\varphi_{n}(\mathrm{rad})$ & $\tau_{n}(\mathrm{ps})$ & $\varphi_{n} \overline{(\mathrm{rad})}$ & $\tau_{n}(\mathrm{ps})$ \\
\hline-1.85 & 0.00 & -1.70 & 0.00 \\
\hline-0.09 & 4.56 & -0.94 & 1.95 \\
\hline 0.64 & 6.43 & -0.10 & 4.13 \\
\hline 1.39 & 8.39 & 0.80 & 6.46 \\
\hline 2.34 & 10.85 & 1.31 & 7.78 \\
\hline-2.91 & 13.51 & 1.85 & 9.17 \\
\hline-2.10 & 15.62 & 2.78 & 11.58 \\
\hline-1.40 & 17.44 & -2.83 & 13.34 \\
\hline-0.55 & 19.62 & -2.69 & 13.68 \\
\hline 0.38 & 22.04 & 3.09 & 12.39 \\
\hline 0.90 & 23.36 & 2.37 & 10.52 \\
\hline 1.01 & 23.67 & 2.02 & 9.64 \\
\hline 0.67 & 22.79 & 1.82 & 9.10 \\
\hline 0.07 & 21.23 & 1.10 & 7.23 \\
\hline-0.12 & 20.75 & 0.35 & 5.29 \\
\hline 0.00 & 21.05 & 0.00 & 4.40 \\
\hline
\end{tabular}




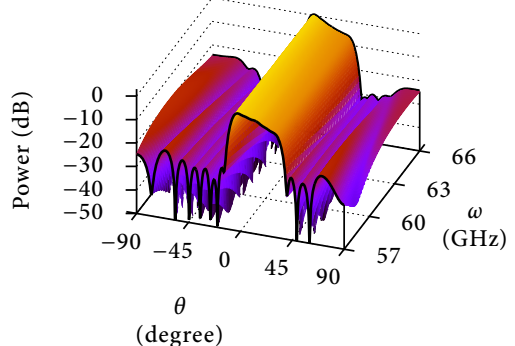

Figure 5.14 - Frequency and time-delay response of $\mathcal{L}_{r}\left(\theta_{d}=15^{\circ}\right)$

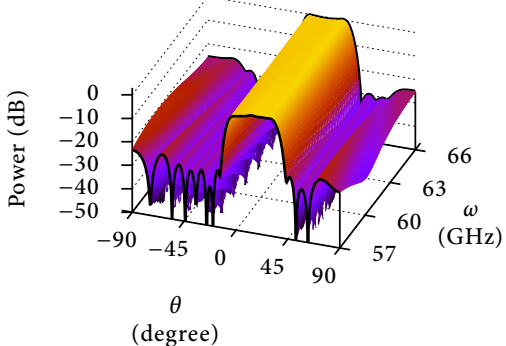

FIGURE 5.15 - Frequency and time-delay response of $\mathcal{L}_{f}\left(\theta_{d}=15^{\circ}\right)$

$$
\begin{aligned}
F(\theta) & =\sum_{n=0}^{N-1} I_{n} e^{j n \frac{2 \pi d}{\lambda} \sin (\theta)} \\
& =\sum_{n=0}^{N-1}\left(\left|I_{n}\right| e^{\varphi_{n}}\right) e^{j 2 \pi \omega\left(n \frac{d}{c} \sin (\theta)\right)} \\
& \equiv \sum_{n=0}^{N-1}\left|I_{n}\right| e^{j 2 \pi \omega\left(n \frac{d}{c} \sin (\theta)+\tau_{n}\right)}
\end{aligned}
$$

Figures 5.14 and 5.15 visualize the power of $\mathcal{L}_{f}$ and $\mathcal{L}_{f}$ expressed through eq. 5.12, for $57 \mathrm{GHz} \leq \omega \leq 66 \mathrm{GHz}$ (figure 1.1). Although not severe, a change in the shape is definitely observable. In addition to being dependent on frequency, the significance of the effect also increases with the scan angle. If this behavior is treated as a ripple defined through figure 3.18, one would expect it to be problematic for the tracking algorithm, especially at the very ends of the chosen frequency range where deviations from the ideal $-10 \mathrm{~dB}$ slope exceed $1 \mathrm{~dB}$. However, the tracking by means of shaped beampatterns detailed in chapter 4 could successfully be repeated for the entire $[57 \mathrm{GHz}, 66 \mathrm{GHz}]$ range as a result of the fact that the deviations manifest themselves mostly as a common convexity or concavity in the shaped beam. Deterioration of the shape at the highest and lowest frequencies did require that the $\Upsilon$ parameter $(\$ 4.2)$, defined to control the ratio of $\mathcal{L}_{f}$ and $\mathcal{L}_{r}$ usage, be set to 5 rather than 10 .

\section{Steering}

Steering (eq. 4.6) changes only the argument of the excitations. In theory this could be an advantage for amplitude tapering. When the element factor is sufficiently uniform, so that the earlier presented countermeasures from $\$ 5.1$ do not have to be 
applied, a single amplitude distribution would be required per pattern shape. In practice this will seldom be the case and tunable/programmable amplifiers are most likely needed for the $\left|I_{n}\right|$ component of the excitations. Delays are unique to each pattern, including the ones that are just steered versions. In that respect, attention must be paid to the fact that narrower beams $(\$ 4.2 .4)$ require larger quantities of different delays to cover a particular angle. This will significantly complicate the beamformer design when $\angle I_{n}$ is implemented as a hardwired true time delay.

\subsection{Conclusions}

Chapter 3 argued that, when a low ripple and/or individually controlled sidelobes are required, Orchard-Elliott synthesis is an attractive way to obtain shaped beampatterns for equispaced linear arrays. However, because it operates on the array factor, the individual antenna gain is assumed to be isotropic. In practice, the (actual) declining gain towards endfire then becomes problematic when shaped beampatterns are to be steered away from broadside. The synthesis algorithm has been modified to take the measured gain of an antenna element into account analytically. This modification comprises the application of a (piecewise) polynomial fit to measured data points, and wrapping this element factor around the visible region during synthesis. Results of synthesizing various differently shaped beampatterns verify that a realistic element factor can be compensated by the excitation values. A $0.1 \mathrm{~dB}$ ripple can still be achieved, which indicates that the proposed modifications have no noteworthy negative effects on the shape of the beampattern. It does entail an increase in computation time, and possibly a larger amplitude range. However, especially within the context of this thesis, it can be said that shaping beampatterns is predominantly a task that is performed off-line, so the additional computation time is not a major drawback. Furthermore, the excitation values for the 16-element array were examined for realization feasibility. Both the $S_{r}$ and $S_{f}$ shape require an amplitude and phase/delay controlled beamformer. Though Orchard-Elliott synthesis does not provide a means to directly control the amplitude range of the excitations, indirectly the roots from the Schelkunoff representation can be repositioned to create different sets of excitations. Often there will be a combination of roots that yields relative amplitude values that can be realized. Less preferred options to (further) reduce this range are allowing a larger ripple or opting for the Taylor sidelobe distribution. For the time delays no particular optimization criterion could be found. Each pattern uses its own set of $N$ delays. Based on that conclusion it can be stated that the number of different beampatterns used (e.g., employing wider beams) should be minimal, otherwise designing the beamforming network will become a complex task. 


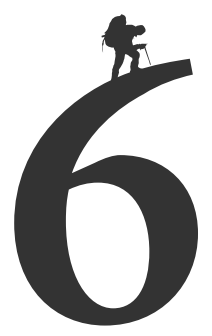

\title{
Planar arrays: ANalysis, SYNTHESIS AND STEERING
}

\begin{abstract}
Fully spatial signal processing (i.e., creating directivity in both elevation and azimuth) would greatly benefit $m m$-wave RF communications. To cover the complete azimuth elevation space, a planar antenna array is required. This chapter investigates the use of planar arrays for shaped-pattern tracking. After having considered various implications of different structural properties, hexagonal shaped arrays are found to be most suitable. Synthesizing a shaped beampattern for this array geometry is, however, far from trivial. An analytically founded synthesis procedure is extended to produce asymmetric beampatterns with very low ripple. The obtained beampatterns are shown to retain their shape well under extreme scan angles, making them useful for shaped-pattern tracking. In addition, the planar synthesis method is a logical extension of Orchard-Elliott synthesis which means that it benefits from all the previously covered material.
\end{abstract}

L INEAR antenna arrays have almost exclusively been the focus in the previous chapters. It was shown how to synthesize shaped beampatterns for this type of array; how to make use of the shape of those patterns for tracking of mobile wireless devices; and what needs to be taken into account before and after implementation. For practical purposes, the use of linear arrays is limited. As one might recall from chapter 2, placing all antenna elements in a single dimension only enhances directionality in one dimension. The use of planar arrays is therefore desired in many practical applications, especially wireless communications. This chapter covers the subjects of the previous chapters in the context of planar arrays.

Before starting with a mathematical analysis of the planar array's response, the shaped patterns that are pursued in this chapter will be introduced first. When all three dimensions are considered, the DoA to be estimated consists of a $\phi$ and a $\theta$ component. That means, where the linear case required two differently shaped beampatterns to find the source's location, a three pattern combination is needed for 
planar arrays. For two of these patterns, figure 6.1 gives a 3-dimensional impression. The first beam shape, denoted $S_{f}$, prescribes constant power over the entire $\left(\Delta \theta_{x}\right.$ by $\Delta \theta_{y}$ ) null-free region. Completely analogous to its linear counterpart, the purpose of $S_{f}$ is to normalize the power level for DoA estimation. The second shape shown describes a ramp surface $S_{r y}$ directly below $S_{f}$. It will not be difficult to see that this ramp can unveil the $\theta$ component with respect to the $y$-axis, hence the $S_{r y}$ designation. A third shape is needed to estimate both the $\theta$ and the $\phi$ angle ${ }^{1}$. This third shape, not shown in the figure, will be a $90^{\circ}$ rotated version of $S_{r y}$. Due to the ramp being oriented orthogonal to $S_{r y}$ (i.e., $\phi+90$ ), it shall be referred to as $S_{r x}$.

For all synthesis purposes, the shapes are defined with respect to the zenith (steering is performed in a later stage). Whilst the center of the beams points towards $\left(0^{\circ}, 0^{\circ}\right)$, the relation between pattern shape and $\phi$ or $\theta$ might not immediately be clear. This is also not a fixed relation. One might find it helpful to consider the silhouettes of $S_{f}$ and $S_{r y}$ at the positive $x$-axis (figure 6.1). The shaded area represents (an ideally shaped) $S_{r y}$ pattern steered to the extreme angle $\left(0^{\circ}, 90^{\circ}\right)$. In this particular situation, $S_{r y}$ provides information about the $\phi$ angle. If the MRA is steered to $\left(90^{\circ}, 90^{\circ}\right)$, it will be $S_{r x}$ that captures $\phi$ whereas the $\theta$ angle can be found with $S_{r y}$. Thus, the role of the patterns will change as the steering angle is adjusted. However, regardless of their labels, sufficient $(\phi, \theta)$ information can always be drawn from two perpendicular ramp shaped patterns when related to the constant power pattern $S_{f}$.

Note that the impressions in figure 6.1 suggest rectangular $\Delta \theta$ boundaries. If the synthesis procedures presented in this chapter are followed, the shape of these boundaries will actually be dictated by the shape of the array. The sidelobe specifications follow later, but can in principle be chosen freely.

\subsection{ANALYSIS}

Recall from chapter 2 that the array factor of an arbitrary planar array is given by

$$
\begin{aligned}
F(\phi, \theta) & =\sum_{n=0}^{N-1} I_{n} e^{j k\left(x_{n} \sin (\theta) \cos (\phi)+y_{n} \sin (\theta) \sin (\phi)\right)} \\
& =\sum_{n=0}^{N-1} I_{n} e^{j k \sin (\theta)\left(x_{n} \cos (\phi)+y_{n} \sin (\phi)\right)}
\end{aligned}
$$

with $k=2 \pi$ and the positions $x_{n}$ and $y_{n}$ expressed proportionally to $\lambda$. Chapter 3 pleaded for the use of regularly structured arrays to enable analytical methods. Although the majority of contemporary planar synthesis techniques aims to generalize for eq. 6.1, this chapter will again focus on synthesis for regularly structured

\footnotetext{
${ }^{1}$ A smooth (synthesizable) surface below $S_{f}$, with at least one point intersecting $S_{f}$ and one point $S_{f}-\Delta P$ (figure 6.1); and in which the power is unique to each $\phi$ and $\theta$ combination, does not exist.
} 


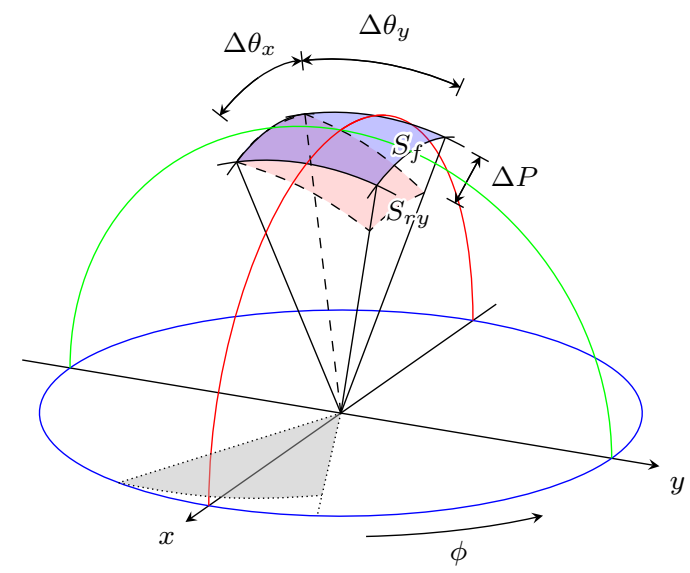

FIGURE 6.1 - 3-D tracking shapes $S_{f}$ and $S_{r y}$ visualized in $(\phi, \theta)$

arrays. The reasoning behind this is the same as before, it is extremely difficult to realize shaped beampatterns with very low ripple, using synthesis techniques that do not discriminate in the array architecture used. This holds even more for planar arrays than for linear arrays.

\section{Array structure}

The two different array structures that are considered in this chapter are:

1. arrays with their elements placed in a rectangular grid

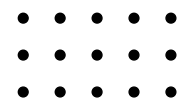

2. arrays with their elements placed in a triangular grid

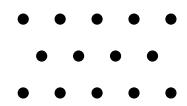

The boundaries (i.e., shape) of the structure can be chosen freely.

First consider the rectangular grid arrangement. A rectangular grid with rectangular boundaries is the most obvious choice for a planar array extension of the previous chapters. The conventions usually adopted for such arrays are shown in figure 6.2. Each row of elements (in the direction of the $y$-axis) has a length of $2 N+1$ while each column contains $2 M+1$ elements ${ }^{2}$. The $m n^{\text {th }}$ element indicates

\footnotetext{
${ }^{2}$ Analysis in case of an even number of rows/columns proceeds in a completely analogous manner, but is less convenient notation wise.
} 


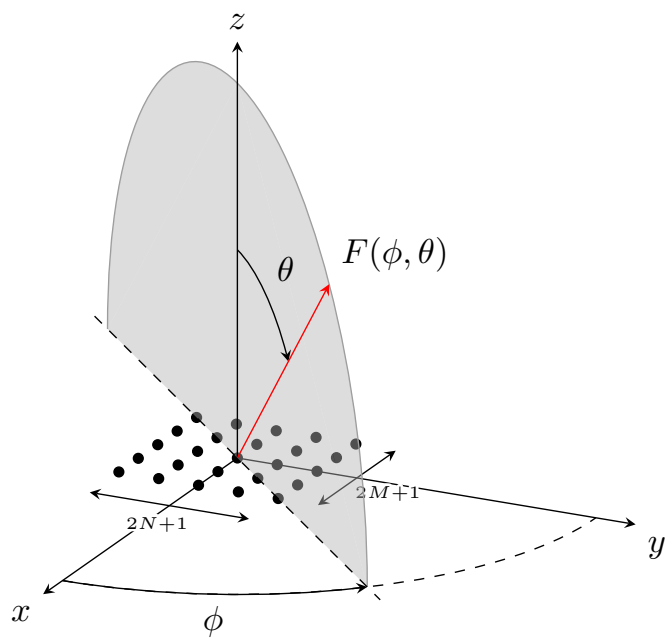

FIGURE 6.2 - Planar array geometry

the element whose position is $\left(m d_{x}, n d_{y}\right)$ relative to the center element. Hereby, $-M \leq m \leq M$ and $-N \leq n \leq N$. Given the property of uniform $d_{x}$ and $d_{y}$ spacing, eq. 6.1 can be written as

$$
F(\phi, \theta)=\sum_{m=-M}^{M} \sum_{n=-N}^{N} I_{m n} e^{j k \sin (\theta)\left(m d_{x} \cos (\phi)+n d_{y} \sin (\phi)\right)}
$$

In literature this is sometimes known as the response of a uniform rectangular array (URA). If in addition $d_{x}=d_{y}=1 / 2 \lambda$, the array may be called a standard rectangular array (SRA) [123].

The notations of eq. 6.2 cannot be adopted for triangular grid arrays. One reason is that not every row has the same number of elements, but more importantly because the $y$ position of the $n^{\text {th }}$ element differs per row $x$. Rather than opting for uniform element separation in the $x$ and $y$ directions, the triangular grid chooses for true equispacing in the $x y$-plane with

$$
d_{x}=\frac{1}{2} \lambda
$$

and

$$
d_{y}=\frac{\sqrt{3}}{4} \lambda
$$


Triangular grid distributions that feature six equally long sides are the main focus of this chapter. This configuration results in a hexagonal shape with uniform $1 / 2 \lambda$ spacing, and is referred to as a standard hexagonal array (SHA) [123]. When discussing SHAs, the index of the elements will be decoupled from their position. Instead of indexing relative to the center element, the index of the $m n^{\text {th }}$ element is determined from the upper leftmost element. An example can be seen in figure 6.7. The corresponding array response is then given by

$$
F(\phi, \theta)=\sum_{m} \sum_{n} I_{m n} e^{j k \sin (\theta)\left(x_{m n} \cos (\phi)+y_{m n} \sin (\phi)\right)}
$$

which is generic like eq. 6.1, but preserves the notion of rows and columns from eq. 6.2. The purpose of that is to provide a convenient indexing scheme for the synthesis techniques developed later. Also note that this more generic notation could be applied to rectangular grid arrays of arbitrary shape.

\section{Steering}

Before looking into synthesizing the proposed shapes, it is important to mention how the (shaped) beampattern of a planar arrayis steered. The synthesis algorithms developed in the following force the designer to define shapes centered around zenith. Steering must be undertaken separately. To this end, let $\left(\phi_{d}, \theta_{d}\right)$ denote the desired MRA position. Any given pattern is then steered to $\left(\phi_{d}, \theta_{d}\right)$ by

$$
I_{m n}=\stackrel{\circ}{I}_{m n} e^{-j k\left(x_{m} \sin \left(\theta_{d}\right) \cos \left(\phi_{d}\right)+y_{m} \sin \left(\theta_{d}\right) \sin \left(\phi_{d}\right)\right)}
$$

where $\stackrel{\circ}{I}_{m n}$ denotes the value of the $m n^{\text {th }}$ excitation before steering. As with the linear case, one can expect this to influence the shape of the pattern to some extent.

\subsection{Synthesis}

Regardless of the strategy chosen, synthesizing a beampattern for planar arrays is widely regarded to be much more difficult than for linear arrays $[26,43,51]$. In an analytical sense, the main reason is that Schelkunoff's theory does not hold in general for a non-linear distribution of antenna elements. In the general case, the planar array factor is a prime polynomial and cannot be factored [24].

\subsubsection{SyNTHESIS VIA SEPARABLE WEIGHTINGS}

The planar array factor of eq. 6.2 does allow factorization under certain conditions. If each row has the same relative excitation distribution, or more precisely when $I_{m n} / I_{m 0}=I_{0 n} / I_{00}$, the array factor is said to be separable $[43,123]$ and can be written as a product of two linear array factors

$$
F(\phi, \theta)=F_{x}(\phi, \theta) F_{y}(\phi, \theta)
$$




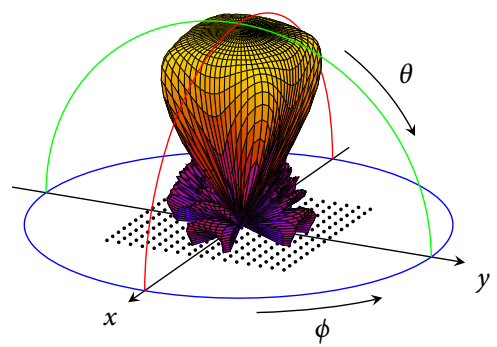

FiguRE 6.3 - Rectangular $S_{f}$ pattern

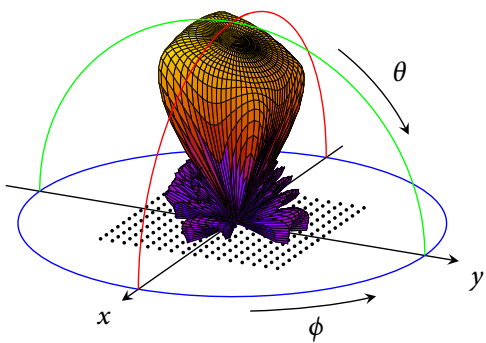

FIGURE 6.4 - Rectangular $S_{r y}$ pattern

wherein

$$
\begin{aligned}
& F_{x}(\phi, \theta)=\sum_{m=-M}^{M} I_{m} e^{j m k d_{x} \sin (\theta) \cos (\phi)} \\
& F_{y}(\phi, \theta)=\sum_{n=-N}^{N} I_{n} e^{j m k d_{x} \sin (\theta) \cos (\phi)}
\end{aligned}
$$

Hereby it holds that

$$
I_{m}=I_{m 0} / I_{00} \quad \text { and } \quad I_{n}=I_{0 n} / I_{00}
$$

In simple terms, when the beampattern of a uniformly spaced rectangular array can be described as a combination of two orthogonal linear beampatterns, its excitations are found by multiplying the corresponding linear excitation distributions

$$
I_{m n}=I_{m} I_{n}
$$

These are in turn equispaced, and can be found with the material from chapter 3 . The only problem with this approach is that one cannot predict how well the pattern will behave when $\phi$ is not $0^{\circ}$ or $90^{\circ}$. In most cases however, eq. 6.11 will give satisfactory results.

Figures 6.3 and 6.4 demonstrate that the shapes as defined in the introduction can indeed be realized on the SRA architecture. In both figures a $16 \times 16$-element array (i.e., SRA-256) was used; the most logical choice given that the ramp and flat shaped $F_{x}$ and $F_{y}$ factors were already computed for $N=16$ in previous chapters. One can immediately observe that both patterns feature a nicely shaped beam with almost no ripple. To make this assessment more detailed, different ripple and sidelobe characteristics are presented in table 6.1. 
TABLE 6.1 - SRA shaped pattern performance

\begin{tabular}{cccrr} 
Shape & Max. Ripple & Avg. Ripple & Max. SLL & Avg. SLL \\
\hline$S_{f}$ & $0.15 \mathrm{~dB}$ & $0.06 \mathrm{~dB}$ & $-30.1 \mathrm{~dB}$ & $-41.5 \mathrm{~dB}$ \\
$S_{r x}$ and $S_{r y}$ & $0.32 \mathrm{~dB}$ & $0.04 \mathrm{~dB}$ & $-30.0 \mathrm{~dB}$ & $-43.7 \mathrm{~dB}$
\end{tabular}

These numbers are the result of inspecting $\phi$-cuts between $0^{\circ}$ and $90^{\circ}$ (in steps of $1^{\circ}$ ), with the ripple evaluated between $\theta=-15^{\circ}$ and $15^{\circ}$. Note that $S_{r x}$ is exactly the same as $S_{r y}$, only $90^{\circ}$ rotated. Although a slightly increased ripple is found compared to the $0.1 \mathrm{~dB}$ of the principal planes, the overall performance is satisfying and well within the tolerance for shaped-pattern tracking (chapter 4). In addition, the obtained sidelobe levels never exceed the $-30 \mathrm{~dB}$ specification.

\section{Steering shaped SRA beampatterns}

Based on the findings in table 6.1, standard rectangular arrays appears to be an ideal candidate for shaped-pattern tracking. That is, until one applies eq. 6.6 to adjust the steering angle. Figure 6.5 illustrates what happens to the shape of the $S_{f}$ pattern when its MRA is changed from $\left(0^{\circ}, 0^{\circ}\right)$ to $\left(0^{\circ}, 35^{\circ}\right)$. Note how the shaped region's angular coverage (indicated by the dashed lines) has changed from square to rectangular. Although the effect is only shown for $S_{f}$ (as it lends itself better for illustration purposes), $S_{r x}$ and $S_{r y}$ experience the same kind of broadening in the general direction of the desired steering angle $\phi_{d}$. The outer sine component in the complex exponential of eq. 6.1 is the main reason for this shape change. Unfortunately that means the cause is fundamental and there is not much that can be done to improve the pattern. In chapter 4 , the linear array response was also subject to broadening whilst tracking. When it is properly taken into account (\$4.3.1), this was not problematic. The same applies for the pattern in figure 6.5.

More severe problems occur when the pattern is also steered away from the principal $x$ and $y$ axes. In figure 6.5, the observant reader may have identified that the beam is stretched out slightly more in $\phi$ directions further away from the $x$-axis. This can be understood by realizing that the distance between the antennas is larger than $1 / 2 \lambda$ if $\phi \neq 0^{\circ}$ or $90^{\circ}$. By increasing the antenna distance, the visible region reduces and effectively the pattern broadens. Figure 6.6 depicts the $S_{f}$ pattern steered to $\left(50^{\circ}, 35^{\circ}\right)$. Here the effect is much more noticeable. In fact, note that the coverage of the SRA now starts to resemble a diamond (equilateral quadrilateral) shape. This is problematic for shaped-pattern tracking. Without consistency in the shapes used for DoA estimation, the angular position of the mobile device being tracked is prone to end up in the sidelobe region due to erroneous steering. This is precisely what the use of shaped patterns had to prevent (chapter 2). Another part of the problem is that the null-free region is shaped squarely. By steering the MRA with the help of eq. 6.6, one does not change the orientation of those square boundaries. To tackle this problem, the patterns would need to be resynthesized with the correct orientation. However, they would no longer be separable and more 


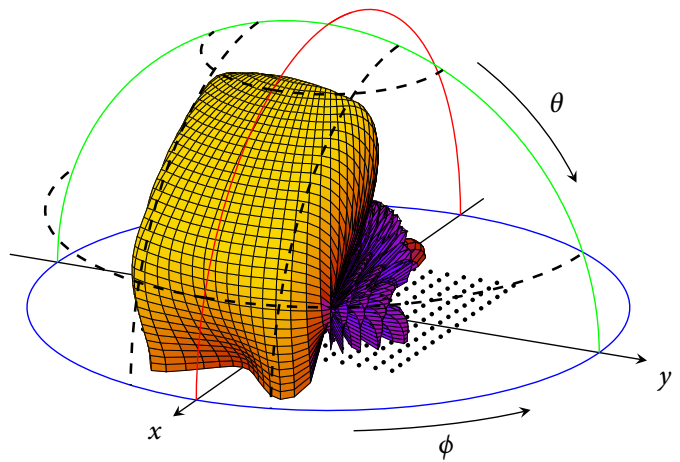

FIgURE 6.5 - Steering the SRA-256 $S_{f}$ pattern over the principal $x$-axis $\left(0^{\circ}, 35^{\circ}\right)$ broadens its shaped region in that direction. The angular area covered by the shaped region changes from square to rectangular.

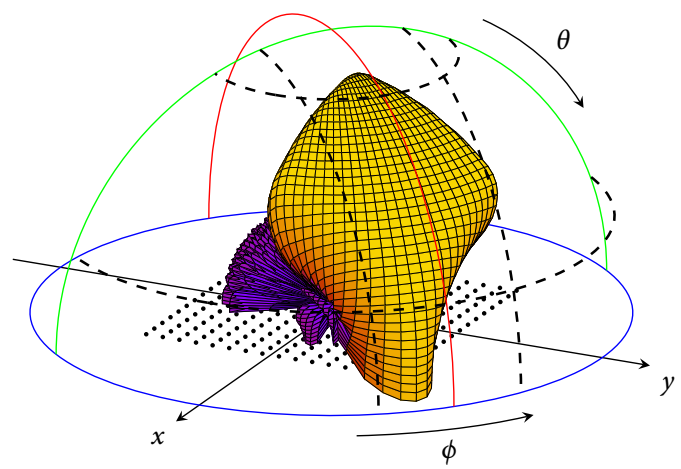

FIGURE 6.6 - Steering $S_{f}$ off the principal axes $\left(50^{\circ}, 35^{\circ}\right)$ results in even more severe warping of the shaped region. The boundaries of the shaped region are now oriented differently, leading to a different angular coverage.

advanced synthesis techniques are then needed. At this point it becomes worthwhile to consider a different array geometry. One that is more suitable for off-axis steering angles, such as the truly equispaced triangular grid array.

\subsubsection{HeXagonal arrays}

Studies in the context of implementing adaptive antenna arrays identify the SHAs as the preferable planar geometry. For example [79], where a comparison is made between (concentric) circular arrays [123] and the SHA. Having simulated the response of both arrays using the method-of-moments, the authors conclude that 
the hexagonal array gives deeper nulls, higher gain and a smaller beamwidth for the same overall array size. Even more convincing is [5], which investigates the performance of a hexagonal distribution of wideband patch antennas for wireless $60 \mathrm{GHz}$ communication. Based on comprehensive simulations and measurements, the hexagonal array is recommended over rectangular arrays. The array described in [5] differs slightly from the SHA. It places the phase center of the elements at $1 / 2 \lambda$ rather than the SHA's element center. The difference can be seen below.

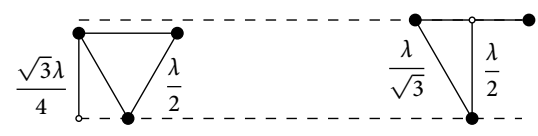

Increasing the element spacing to $\lambda / \sqrt{3}$ results in less mutual coupling, which makes this array perform better in certain aspects [5]. It is also argued in [5] that the array factor will not experience disturbing spatial aliases by doing so. Because both arrays are proportionally similar, one may assume the standard $1 / 2 \lambda$ spacing during synthesis, and take advantage of the $\lambda / \sqrt{3}$ spacing by increasing the distance during the implementation phase.

Figure 6.7 depicts a 169-element SHA (SHA-169), which serves as an example throughout the next sections. Aside from the previously mentioned benefits, particularly interesting structural properties are its truly equispaced antenna positions and the 6-fold rotational symmetry. Equispacing will make broadening of a pattern's shaped region (experienced when the elevation angle is increased) more uniform for different azimuth angles. The rotational symmetry is beneficial for the orientation (i.e., the shape of the null free region) of the patterns. For the second argument, consider that a shaped beam pointing to the zenith has a particular orientation with respect to the $x-y$ frame of reference (given that it is not $\phi$-invariant). By rearranging the array excitations, this orientation can be changed to any of the rotational symmetric axes (e.g., from $x$ and $y$ to $\hat{x}$ and $\hat{y}$ in figure 6.7), without affecting the pattern's shape. Such axes shall be referred to as pseudo axes. The hexagonal array is rotational symmetric every $60^{\circ}$, the SRA only every $90^{\circ}$. Moreover, when it is considered that for all practical purposes $S_{r x}$ and $S_{r y}$ are equivalent when rotated $90^{\circ}$, the beampattern can be reproduced exact every $30^{\circ}$. This greatly reduces the need for steering the MRA to $\phi$ angles far away from the main/pseudo axes.

The most notable downsides of the hexagonal array are its generally higher sidelobe levels [5] and that the excitations are no longer separable. Synthesizing the shaped patterns is therefore much more difficult.

\subsubsection{RELATED WORK}

When literature is consulted for SHA beampattern synthesis, one will predominantly find the use of techniques which work for any array configuration. Some examples of contemporary work are [78], in which a GA is used, and [115], where 


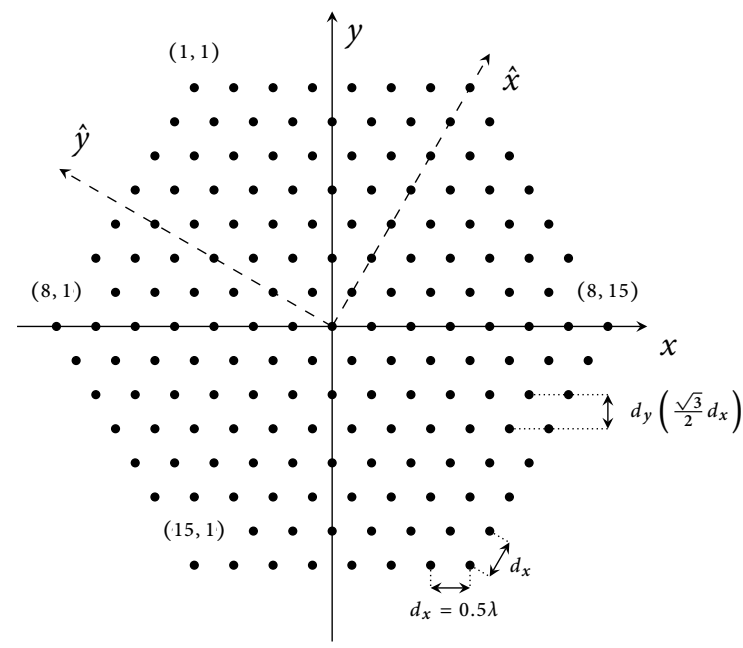

FIGURE 6.7 - SHAs feature equally spaced antenna elements and a high degree of rotational symmetry. The 15 elements in the center row dictate the remainder of the element positions and have been chosen such that the overall array size is comparable to the SRA-256.

SA and PSO are applied to a 61-element SHA. Recall that these methods were also considered for linear array synthesis in \$3.5. Properly constraining the ripple in the shaped region proved to be difficult even in the linear case. Planar arrays add difficulties because of the inherently large number of degrees of freedom involved [127]. However, the popularity of global optimization is logical given the continuous advances in (parallel) computing power. One generic synthesis algorithm would indeed be the antenna designer's ultimate tool. Global optimization theoretically allows for full wave simulation in each iteration, such that every possible effect on the pattern's shape can be taken into account. In practice, the computational complexity remains daunting. To give an indication, a cluster supercomputer was used in [127] to synthesize a flat-top beam (e.g., $\left.S_{f}\right)$ for $10 \times 10$ rectangular arrays. A similar effort was made for the SHA-169 on a smaller scale, using the Matlab global optimization toolbox. As expected, a pool of six threads running on a $3.40 \mathrm{GHz}$ i7-26oo CPU did not lead to a desirable pattern after 72 hours of optimization. The ripple of the obtained patterns was much worse than in the linear pattern, with nulls frequently dipping below $-20 \mathrm{~dB}$ in the shaped region. This indicates that global optimization is still impractical for low ripple shaped patterns.

The alternating projections method [25] has also been shown to work for planar arrays, although the examples that were chosen are relatively simple. While alternating projections work reasonably well for linear shaped patterns; it is unknown if that is also true for planar arrays. Due to the approach being vastly different 
from the work presented earlier in this thesis, no experiments were conducted. The same applies to the least squares solutions, even though the examples in [126] and [64] are remarkably similar to the $S_{r x} / S_{r y}$ shapes desired here. The ripple in those examples is estimated to be in the order of a couple of decibels.

With the exception of [57], very little analytical work targets hexagonal arrays specifically. For [57] to work, the shape of the desired pattern needs to be $\pi / 3$ periodic. This would not be problematic for $S_{f}$ but the other shapes obviously do not meet this requirement. In general analytical solutions assume some form of symmetry to simplify the problem. The results do tend to feature the desired low ripple, even in the planar case. The number of options for $S_{f}$ synthesis is still quite extensive. One of the reasons might be that this contour, known in literature as a footprint pattern, has an important role to fulfill in satellite broadcasting [11]. Sophisticated techniques [102][3] are capable of producing footprint pattern with very complex boundaries. However, the specific combination of a low ripple and non-flat power response has never been covered.

In the recommendations section of [91], Orchard et al. mention the principle of collapsed distributions as a possible extension to planar arrays. Planar synthesis based on this principle is rarely encountered. In [13] and subsequently [69], a footprint pattern was successfully realized for the rectangular grid array. The only other (independent) work based on collapsed distributions was found in [23]. Here a circular footprint pattern is synthesized using a circular array. Because the method of collapsed distributions associates nicely with the previously presented material, it is redefined to include triangular grid arrays (e.g., the SHA-169) in the next section. Following in $\$ 6.4$, all previously needed assumptions on symmetry are also lifted such that the $S_{r x}$ and $S_{r y}$ patterns can also be synthesized.

\subsection{SyNTHESIS VIA COLLAPSED DISTRIBUTIONS}

The principle of collapsed distributions $[41,43]$ relies on the transformation of a planar array into an equivalent collapsed linear array. It can be shown that, when the (complex) excitation values are preserved, the response of this collapsed distribution matches the response of the planar array. This equivalence leads to a planar synthesis procedure that consists of the following proceedings:

1. Collapsing the planar array over a chosen azimuth angle $\phi_{c}$, to obtain its equivalent linear array;

2. Invoking a linear synthesis algorithm to synthesize the 2-D shape dictated by a $\phi$-cut of the full $(\phi, \theta)$ specification of the desired $3-D$ pattern shape;

3. Reversing the process by spreading the excitations of multiple collapsed distributions to their original planar distribution.

Collapsing the planar array starts by choosing the azimuth angle $\phi_{c}$ over which to collapse. This angle often needs to be chosen carefully, as the size and geometry 
of the resulting linear array strongly depend on it. In order to utilize for example the techniques developed in the previous chapters, uniform spacing is required. Assuming that the desired pattern is fully specified in $(\phi, \theta)$, a $\phi$-cut matching to $\phi_{c}$ can be derived for linear array synthesis. This synthesis is based on the previous chapters. By repeating this act for sufficient $\phi_{c}$ angles, the pattern of the planar array will be constrained through the shapes synthesized for its collapsed distributions. The remaining task is then to reverse the collapsing process. For the arrays of interest in this thesis, that will be accomplished by solving a system of equations. Instead of immediately applying these steps to the 169-element hexagonal array, they will first be detailed further with the help of a simpler example.

\subsubsection{COLLAPSING PLANAR ARRAYS}

Consider the rectangular array in figure 6.8, and let its $m n^{\text {th }}$ element position (eq. 6.2) be denoted in vector notation by $\left[\begin{array}{ll}x_{m n} & y_{m n}\end{array}\right]^{T}$. A planar rotation $\alpha$ is introduced to all positions. Like $\phi, \alpha$ is defined relative to the positive $x$-axis. The new element positions $\left(x_{m n}^{\prime}, y_{m n}^{\prime}\right)$ are found by

$$
\left[\begin{array}{l}
x_{m n}^{\prime} \\
y_{m n}^{\prime}
\end{array}\right]=\left[\begin{array}{rr}
\cos (\alpha) & -\sin (\alpha) \\
\sin (\alpha) & \cos (\alpha)
\end{array}\right]\left[\begin{array}{l}
x_{m n} \\
y_{m n}
\end{array}\right]
$$

which is easily verified by representing the $x$ and $y$ positions as a complex number

$$
\begin{aligned}
z & =x+j y \\
& =e^{j \phi}
\end{aligned}
$$

with $\phi=\arg (z)$. The rotation $\alpha$ can be expressed by

$$
\begin{aligned}
e^{j(\phi+\alpha)} & =e^{j \phi} e^{j \alpha} \\
& =z e^{j \alpha}
\end{aligned}
$$

Using Euler's formula, eq. 6.14 is expanded

$$
\begin{aligned}
z e^{j \alpha} & =(x+j y) e^{j \alpha} \\
& =(x+j y)(\cos (\alpha)+j \sin (\alpha)) \\
& =x \cos (\alpha)+j x(\sin (\alpha)+j y \cos (\alpha)-y \sin (\alpha) \\
& =(x \cos (\alpha)-y \sin (\alpha))+j(x \sin (\alpha)+y \cos (\alpha))
\end{aligned}
$$

from which it follows that 


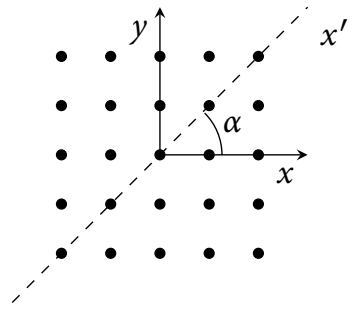

FIGURE 6.8 - Before rotation



FIGURE 6.9 - After rotation

$$
\begin{aligned}
& x^{\prime}=x \cos (\alpha)-y \sin (\alpha) \\
& y^{\prime}=x \sin (\alpha)+y \cos (\alpha)
\end{aligned}
$$

The result of this rotation is shown in figure 6.9, for $\alpha=45^{\circ}$. Essentially nothing changes except that the frame of reference for the element positions is different. The positions are now expressed relative to $x^{\prime}$ (and $y^{\prime}$ ) so that eq. 6.2 may be reformulated

$$
F(\phi, \theta)=\sum_{m} \sum_{n} I_{m n} e^{j k \sin (\theta)\left(x_{m n}^{\prime} \cos (\phi-\alpha)+y_{m n}^{\prime} \sin (\phi-\alpha)\right)}
$$

This will appear as an unnecessary complication, but when the condition $\alpha=\phi$ is met, eq. 6.18 becomes

$$
F\left(\theta_{x^{\prime}}\right)=\sum_{m} \sum_{n} I_{m n} e^{j k x_{m n}^{\prime} \sin \left(\theta_{x^{\prime}}\right)}
$$

where $\theta_{x^{\prime}}$ is the elevation angle measured in the $x^{\prime} z$-plane. Note that by choosing $\alpha=\phi$, the $y$-factor of the element positions is eliminated from the equation. One can imagine this as if the elements were projected (i.e., collapsed) onto the $x^{\prime}$-axis over a line perpendicular to $x^{\prime}$. Figure 6.10 illustrates this projection graphically. Mathematically, eq. 6.19 is equivalent to eq. 2.20 (i.e., the array factor of a linear antenna array). This holds for conventional patterns, but also for more complicated cases such as shaped beampatterns [41]. As correctly remarked by [23], a possible purpose for the collapsed distribution could therefore also be fast evaluation of a complex shaped planar beampattern in several key azimuth angles.

Figure 6.12 may help develop some intuition for the above. Shown is a crosscut of the $y$-oriented ramp pattern that was previously obtained by separable weightings for the SRA-256 array. The highlighted part in the $\theta_{x}^{\prime}$ plane is what one will be looking at when the array is collapsed to $63.435^{\circ}$ and eq. 6.19 evaluated for $\theta_{x}^{\prime}=-90^{\circ} \ldots 90^{\circ}$. 


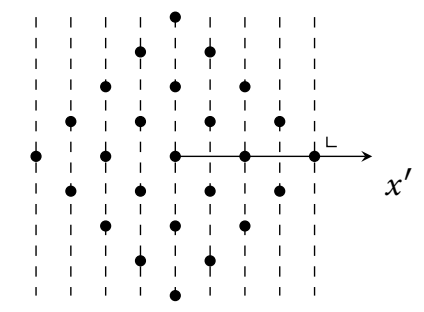

FigURE 6.10 - Projection onto the $x^{\prime}$-axis
FIgURE 6.11 - Collapsed equivalent linear array

\subsubsection{COLLAPSED DISTRIBUTIONS AND LINEAR SYNTHESIS}

The planar array can theoretically be collapsed to any chosen $\phi_{c}$ and it will be possible to synthesize a pattern for it. However, in chapter 3 it was determined that Orchard-Elliott synthesis [91] is the preferred method, and it requires that the antenna elements are equispaced. Another restriction to consider is that the process eventually needs to be reversed for planar synthesis. In essence this means that there are two factors that need to be taken into account when choosing a $\phi_{c}$ angle for collapsing:

» The resulting collapsed distribution and the linear array synthesis method to be used must be compatible.

» The $\phi_{c}$ angles should preferably be distributed evenly over the azimuth, to control the shape of the planar pattern as well as possible.

With respect to spreading the collapsed distributions (discussed next) it is also good to keep the number of elements in the collapsed distributions small.

Consider again the small $5 \times 5$ SRA example. Figure 6.11 shows that the equivalent linear array for $\phi_{c}=45^{\circ}$ is equispaced and consists of 9 elements. It is not difficult to see that when the rectangular array is collapsed onto its principal axes (i.e., $\phi=0^{\circ}$ and $\phi=90^{\circ}$ ), a $1 / 2 \lambda$ spaced array of 5 elements will result. In light of the desire for evenly distributed $\phi_{c}$ angles, $22.5^{\circ}$ might be the next logical choice. However, figures 6.13 and 6.14 illustrate why this is a poor choice. Clearly the obtained geometry is not suitable for the synthesis method of choice. Furthermore, note that all the planar elements end up taking a unique position on the $x^{\prime}$-axis. This is bad for the system of equations to be solved while spreading the $\phi_{c}$ distributions. A large numbers of elements in the collapsed distribution is therefore often a good indication that it has little value. When not all $\phi$ angles work well for the collapsed distribution principle, one will undoubtedly question which angles are suitable. For planar arrays that have their elements placed arbitrarily, it is difficult to determine a priory what $\phi_{c}$ angles yield a useful collapsed distribution. With the presence of some regularity (e.g., rectangular or triangular grid structures), selections of angles 


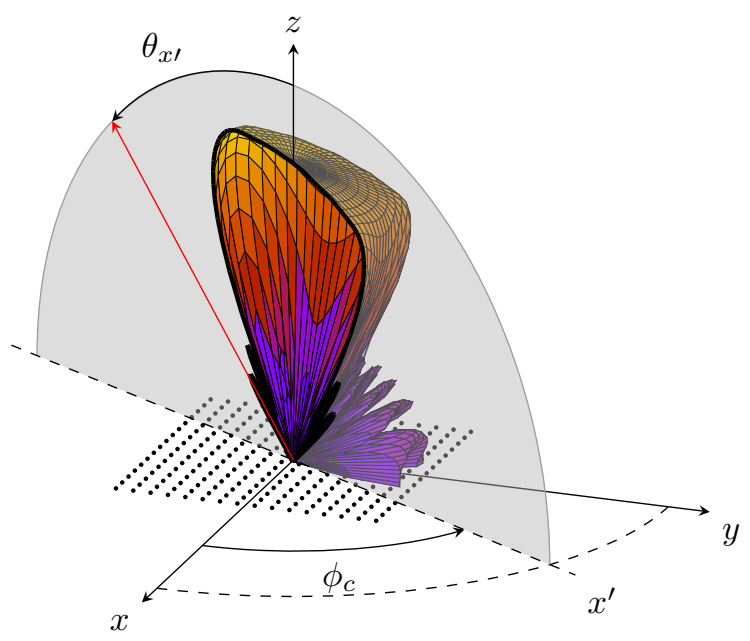

FIGURE 6.12 - The response of a collapsed equivalent linear array is identical to the planar array's response in the $x^{\prime} z$-plane (i.e., a $\phi$-cut). Shown here for the $16 \times 16$ SRA $S_{r y}$ pattern evaluated at $\phi_{c}=63.435^{\circ}$.

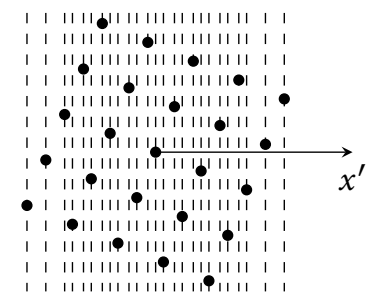

FIGURE 6.13 - Projection $\left(22.5^{\circ}\right)$

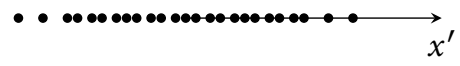

FIGURE 6.14 - Collapsed array $\left(22.5^{\circ}\right)$

that meet certain requirements can be predefined. An example of such a selection is table 6.2, which basically summarizes a comprehensive study of rectangular arrays performed in [69]. A similar list will also be compiled for the SHA geometry in $\$ 6.4$.

TABLE 6.2 - Equispaced collapsed distributions for SRAs ( $d_{x^{\prime}}$ denotes spacing)

\begin{tabular}{cc}
$\phi_{c}$ & $d_{x^{\prime}}$ \\
\hline $0^{\circ}$ & $0.500 \lambda$ \\
$14.036^{\circ}$ & $0.121 \lambda$ \\
$26.565^{\circ}$ & $0.224 \lambda$ \\
$33.690^{\circ}$ & $0.139 \lambda$ \\
$45^{\circ}$ & $0.350 \lambda$
\end{tabular}




\section{Linear synthesis}

Once sufficient equispaced collapsed distributions have been found, linear synthesis is fairly straightforward. The 2-D shape $S(\theta)$ to be synthesized is derived from $S(\phi, \theta)$ by substituting $\phi_{c}$ for $\phi$. Often this means combining two azimuth angles, because the elevation angle of $S(\phi, \theta)$ is limited to $0^{\circ} \ldots 90^{\circ}$. For simplicity, let

$$
S\left(\phi_{c}, \theta\right)=\left\{\begin{array}{clrl}
S\left(\phi_{c}+180^{\circ}, 90^{\circ}+\theta\right) & \text { if } & -90^{\circ} \leq \theta<0^{\circ} \\
S\left(\phi_{c}, \theta\right) & \text { if } & 0^{\circ} \leq \theta \leq 90^{\circ}
\end{array}\right.
$$

therefore be denoted by $S_{\phi_{c}}$. If desired, symmetric patterns such as the flat-top beam can be obtained with the real valued synthesis procedure by Kim [72]. For asymmetric $S_{\phi_{c}}$, the $I_{n}$ will always be complex such that they need to be synthesized with the original method by Orchard and Elliott, or the modified version from chapter 5 .

\subsubsection{PlanAR SYNTHESIS BY SPREADING COLLAPSED DISTRIBUTIONS}

The reverse of collapsing a planar array is known as spreading its collapsed distributions. Which strategy to use when spreading out collapsed distributions depends on the type of planar array. With concentric circular arrays, a Fourier or Taylor series expansion has been shown to work well [23]. When dealing with grid array structures, a system of equations needs to be solved. Let the array factor of each linear distribution, collapsed on $\phi_{c}$, therefore be represented by

$$
F_{\phi_{c}}(\theta)=\sum_{l=1}^{L} I_{l} e^{j k d_{x^{\prime}} l \sin (\theta)}
$$

with $L$ the number of elements, the usual substitution $k=\frac{2 \pi}{\lambda}$ and an inter-element spacing $d_{x^{\prime}}$ (according to e.g., table 6.2). As figures 6.10 and 6.11 depicted, multiple excitations $I_{m n}$ contribute to one linear excitation $I_{l}$ if the $\phi_{c}$ angle is chosen correctly. More precisely, when for the $m n^{\text {th }}$ element of the planar array holds that

$$
n d_{x} \cos \left(\phi_{c}\right)-m d_{y} \sin \left(\phi_{c}\right)=d_{x^{\prime}} l
$$

this element contributes to the $l^{\text {th }}$ element of the equivalent linear array. One can thus write the equation

$$
\sum_{m=1}^{M} \sum_{n=1}^{N} \epsilon_{m n} I_{m n}=I_{l}
$$

with 


$$
\epsilon_{m n}= \begin{cases}1 & \text { if } \frac{n d_{x} \cos \left(\phi_{c}\right)-m d_{y} \sin \left(\phi_{c}\right)}{d_{x^{\prime}}}=l \\ 0 & \text { otherwise }\end{cases}
$$

By applying eq. 6.23 to all $I_{l}$ from eq. 6.21, a (sub)system of equations is obtained. The complete system of equations is formed by applying eq. 6.23 to the excitations $I_{\phi_{c}, l}$ from every collapsed distribution:

$$
\sum_{m=1}^{M} \sum_{n=1}^{N} \epsilon_{m n} I_{m n}=I_{\phi_{c}}, l
$$

Denoting this as

$$
\mathbf{A x}=\mathbf{b}
$$

the coefficients of $\mathbf{A}$ represent $\epsilon_{m n}$, $\mathbf{b}$ the excitations $I_{\phi_{c}, l}$ found for the collapsed distributions and $\mathbf{x}$ the unknown excitations $I_{m n}$ of the planar array.

\section{Solving the system of equations}

If $\mathbf{A}$ is square, the unknown $x$ values can be found by matrix inversion. This is however seldom the case. Overdetermination is much more likely to occur, either because there is symmetry that can be exploited or because eq. 6.25 comprises many collapsed distributions. Overdetermined systems are solved in a least squares sense, minimizing the norm $\|\mathbf{A x}-\mathbf{b}\|^{2}$. When an underdetermined system is encountered (or when the result deviates too much from the desired response), additional collapsed distributions should be added if possible.

Recall from chapter 3 that asymmetric pattern shapes require complex excitation values. The synthesis of $S_{r x}$ and $S_{r y}$ therefore demands the use of complex valued systems. This has never been attempted by any preceding work, but $\$ 6.4$ will show that (in spite of a slightly higher error in the $l^{2}$-norm) it works very well. Furthermore, [11] and [69] specifically mention singular value decomposition (SVD) to solve the system of equations. However, equally satisfying results were obtained using alternative solvers based on QR-decomposition or the pseudoinverse.

\subsubsection{EXAMPLE: PENCIL BEAM WITH $\phi$-INVARIANT SIDELOBES}

A simple demonstration of spreading collapsed distributions (derived from an example in [43]) is presented next. The objective will be to synthesize a pencil beam with sidelobes that are both uniform in height and $\phi$-invariant (i.e., ring sidelobes). In literature this combination is known as a Tseng-Cheng pattern $[52,73]$, or as a Dolph-Chebychev pattern [39] in the linear case. The targeted array will be the small $5 \times 5$ SRA, for which quadrant symmetry can be assumed. Let its (normalized) excitations therefore be represented as shown in figure 6.15 . 


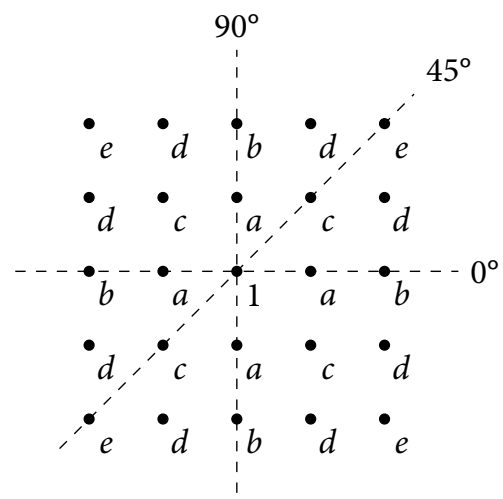

FIGURE 6.15 - Collapsing a $5 \times 5$ SRA with quadrantal symmetry

Collapsing the array onto the $x$-axis $\left(\phi_{c}=0^{\circ}\right)$ yields the following $1 / 2 \lambda$-spaced linear distribution:

$$
(b+2 d+2 e)(a+2 c+2 d)(1+2 a+2 b)(a+2 c+2 d)(b+2 d+2 e)
$$

Collapsed onto the $y$-axis, one obtains exactly the same distribution which does not benefit the system of equations. However, when the array is collapsed onto $\phi_{c}=45^{\circ}$, one finds a 9-element array with the excitations:

$$
(e)(2 d)(2 b+c)(2 a+2 d)(1+2 c+2 e)(2 a+2 d)(2 b+c)(2 d)(e)
$$

and a spacing of $0.35 \lambda$. Knowing the size and structure of both collapsed arrays, an appropriate synthesis technique can be selected. The first procedure by Elliott [40] (\$3.2) lends itself perfectly for the type of pattern needed here: a main lobe at $0 \mathrm{~dB}$ with its surrounding sidelobes suppressed uniformly. Given the small array size, the SLL shall be set to $-16 \mathrm{~dB}$.

Letting the normalized excitations of the collapsed distributions be represented by

$$
x_{1} x_{2} 1 x_{2} x_{1}
$$

and

$$
z_{1} z_{2} z_{3} z_{4} 1 z_{4} z_{3} z_{2} z_{1}
$$

one finds 


$$
\begin{aligned}
& x_{1}=0.690-0.007 j \\
& x_{2}=0.865-0.004 j
\end{aligned}
$$

and

$$
\begin{aligned}
& z_{1}=0.923-0.011 j \\
& z_{2}=0.691-0.006 j \\
& z_{3}=0.852-0.005 j \\
& z_{4}=0.962-0.003 j
\end{aligned}
$$

by performing the said synthesis. The pattern of the planar array will now be forced to match the shape of the collapsed distributions in the two $\phi_{c}$-cuts when

$$
\begin{aligned}
(b+2 d+2 e) & =x_{1} \\
(a+2 c+2 d) & =x_{2} \\
(1+2 a+2 b) & =1 \\
(e) & =z_{1} \\
(2 d) & =z_{2} \\
(2 b+c) & =z_{3} \\
(2 a+2 d) & =z_{4} \\
(1+2 c+2 e) & =1
\end{aligned}
$$

Due to eq. (6.27) and (6.28), this system can be simplified to:

$$
\begin{aligned}
(b+2 d+2 e) & =x_{1}(1+2 a+2 b) \\
(a+2 c+2 d) & =x_{2}(1+2 a+2 b) \\
(e) & =z_{1}(1+2 c+2 e) \\
(2 d) & =z_{2}(1+2 c+2 e) \\
(2 b+c) & =z_{3}(1+2 c+2 e) \\
(2 a+2 d) & =z_{4}(1+2 c+2 e)
\end{aligned}
$$

However, it is still not properly dimensioned. In most cases one should proceed with a least squares approximation. In this particular case, such an approach will not work well due to the small number of unknowns. Instead, the equations 


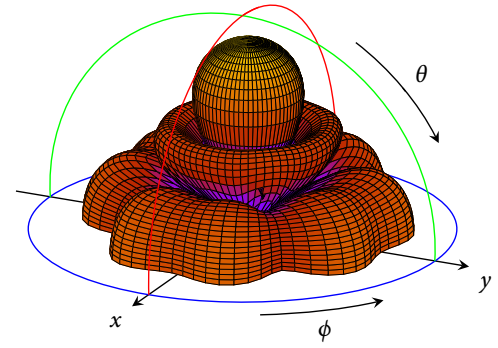

FIGURE 6.16 - Ring sidelobes

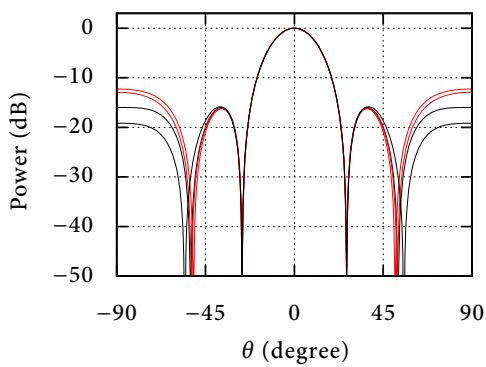

FIGURE $6.17-\phi$-cuts at $0^{\circ}, 15^{\circ}, 30^{\circ}, 45^{\circ}$

for the outermost (least influential) excitations [74] of the largest collapsed distributions can be omitted to obtain a deterministic system of equations. Leaving $(e)=z_{1}(1+2 c+2 e)$ out of the equation gives a square matrix

$$
\begin{aligned}
\left(1-2 x_{1}\right) a+\left(1-2 x_{1}\right) b+2 d+2 e & =x_{1} \\
\left(1-2 x_{2}\right) a-2 x_{2} b+2 c+2 d & =x_{2} \\
-2 z_{1} c+2 d-2 z_{2} e & =z_{2} \\
2 b+\left(1-2 z_{3}\right) c-2 e & =z_{3} \\
2 a-2 z_{4} c+2 d-2 z_{4} e & =z_{4}
\end{aligned}
$$

that, after inversion, yields the following planar excitations:

$$
\begin{aligned}
& a=0.242+0.002 j \\
& b=0.566-0.006 j \\
& c=0.392-0.002 j \\
& d=0.618-0.008 j \\
& e=0.002-0.001 j
\end{aligned}
$$

The result can be observed in figure 6.16. In addition figure 6.17 shows various $\phi$ intersections of the pattern. The sidelobe behavior is almost $\phi$-invariant, but not entirely. As to be expected, values higher than $16 \mathrm{~dB}$ are obtained for $\phi$ angles not included as a collapsed distribution. For larger arrays, it is therefore generally a good idea to include more $\phi_{c}$ angles. The lower SLL at endfire, that is found for $\phi=45^{\circ}$, can be explained by the fact that the corresponding collapsed distribution could only be included partially. 


\subsection{TraCKING PATTERNS FOR HEXAGONAL ARRAYS}

Now that the basic concept of collapsed distributions has been established, it shall be applied to the hexagonal array proposed in \$6.2.2. This involves a few non-trivial steps in addition to the ones already explained. Preceding work (from the literature) has always assumed that the arrays/excitations/patterns exhibited at least quadrant symmetry. By doing so, the response of each collapsed distribution is implicitly made suitable for spreading. Obviously this assumption can no longer be satisfied for $S_{r x}$ and $S_{r y}$. Consequently, the excitations of their collapsed distributions have to be explicitly made suitable. When Orchard-Elliott synthesis is employed, this preparation consists of:

"Specific placement (i.e., balancing) of the roots in the Schelkunoff representation (eq. 3.10).

»Applying a common normalization to the power pattern of the individual collapsed distribution - and possibly shaping the pattern relative to the MRA instead of its peak value.

Furthermore, collapsing the SHA-169 yields linear distributions that are considerably larger than those of the $5 \times 5$ SRA example. One may encounter problems with the expansion of polynomials belonging to some of the larger collapsed distributions. In spite of the SHA-169's size not being excessively large compared to related works (e.g., $[11,69])$, this problem was never identified or mentioned. Possible solutions and workarounds will therefore also be detailed in this section.

The way in which the above subjects will be treated is by following the collapsed distribution flow as explained in $\$ 6.3$, while elaborating on the particularities for asymmetric patterns and large polynomials when they are encountered.

\subsubsection{EQUISPACED LINEAR DISTRIBUTIONS}

The first matter to be addressed is finding equispaced collapsed distributions for the standard hexagonal array. An extensive selection of $\phi_{c}$ angles, each fulfilling the uniform spacing condition, has been listed in table 6.3. For each $\phi_{c}$ in table 6.3, a similar collapsed distribution can be found at $\phi_{c}+90^{\circ}$. However, these will not be needed for $S_{r x}$ and $S_{r y}$ because there is still symmetry to be exploited over one axis.

\subsubsection{LINEAR SYNTHESIS}

Next, the shape impressions from figure 6.1 are formalized for synthesis. To remain consistent with the power difference used for DoA estimation in chapter 4 , the power decay of the ramp $(\Delta P)$ is specified as $-10 \mathrm{~dB}$, the same $40^{\circ}$ wide shaped region $(\Delta \theta)$ is desired and the height of the sidelobes is also set to $-30 \mathrm{~dB}$. Although figure 6.1 depicted the shaped regions with rectangular azimuth boundaries, $\Delta \theta$ shall be kept $\phi$ invariant for the shapes defined here. The shape of the beam will 
TABLE 6.3 - Equispaced collapsed distributions for triangular grid arrays. $L$ is the number of elements specifically for the proposed 169 -element standard hexagonal array.

\begin{tabular}{ccc}
$\phi_{c}$ & $d_{x^{\prime}}$ & $L$ \\
\hline $0^{\circ}$ & $0.250 \lambda$ & 29 \\
$10.89^{\circ}$ & $0.164 \lambda$ & 43 \\
$16.10^{\circ}$ & $0.121 \lambda$ & 57 \\
$19.11^{\circ}$ & $0.094 \lambda$ & 71 \\
$30^{\circ}$ & $0.433 \lambda$ & 15 \\
$40.89^{\circ}$ & $0.094 \lambda$ & 71 \\
$43.90^{\circ}$ & $0.121 \lambda$ & 57 \\
$49.11^{\circ}$ & $0.164 \lambda$ & 43 \\
$60^{\circ}$ & $0.250 \lambda$ & 29 \\
$70.89^{\circ}$ & $0.164 \lambda$ & 43 \\
$76.10^{\circ}$ & $0.121 \lambda$ & 57 \\
$81.05^{\circ}$ & $0.078 \lambda$ & 85 \\
$90^{\circ}$ & $0.433 \lambda$ & 15
\end{tabular}

then be dictated by the shape of the array (i.e., hexagonal rather than rectangular), which turns out beneficial for the ripple.

The full $(\phi, \theta)$ shape of the flat-top beam is defined as

$$
S_{f}(\phi, \theta)=\left\{\begin{array}{rll}
0 & \text { if } & |\theta| \leq 20^{\circ} \\
-30 & \text { if } & |\theta|>20^{\circ}
\end{array}\right.
$$

with $S_{f}$ expressed in decibels. The $x$-oriented ramp shape is then given by

$$
S_{r x}(\phi, \theta)=\left\{\begin{array}{lll}
-\frac{1}{4} \theta\left(1-\frac{\phi}{90^{\circ}}\right) & \text { if } & |\theta| \leq 20^{\circ} \text { and } 0^{\circ} \leq \phi \leq 90^{\circ} \\
-30 & \text { if } & |\theta|>20^{\circ}
\end{array}\right.
$$

and its $y$-oriented counterpart as

$$
S_{r y}(\phi, \theta)=\left\{\begin{array}{lll}
-\frac{1}{4} \theta\left(\frac{\phi}{90^{\circ}}\right) & \text { if } & |\theta| \text { and } 0^{\circ} \leq \phi \leq 90^{\circ} \\
-30 & \text { if } & |\theta|>20^{\circ}
\end{array}\right.
$$

A 2-D shaping function for Orchard-Elliott synthesis follows by substituting $\phi$ with its corresponding collapsed distribution angle $\phi_{c}$. Due to its $\phi$-invariance, $S_{f}$ will always give the same flat response in region I. The other functions, on the other hand, prescribe a slope of which the steepness gradually increases/decreases with $\phi$. Note that $S$ is always zero at $\theta=0^{\circ}$. This is to accommodate the explicit normalization step for the collapsed distribution responses of $S_{r x}$ and $S_{r y}$.

Recall from $\$ 3.4 .1$ that the beamwidth is largely determined by the number of roots $\left(w_{n}\right)$ in 


$$
F(w)=I_{N} \prod_{n=1}^{N-1}\left(w-w_{n}\right)
$$

that are positioned off the unit circle. Table 6.3 reveals that the visible region is frequently smaller than $180^{\circ}$ (i.e. $d_{x^{\prime}}<0.5 \lambda$ ). One might expect this needs to be reflected in the number of roots placed off the unit circled. However, the visible region (and thus region I) is proportional to the number of elements in the collapsed distribution. Due to this almost fixed ratio, always four roots need to be placed off the unit circle to create a null-free region of roughly $40^{\circ}$. As mentioned earlier, the shape of the antenna array then dictates the resulting shaped region boundaries. Forcing the null-free region to take on a different shape is possible by varying the number of off-circle roots. However, this will increases the ripple of the pattern (up to several decibels).

Now the difference between symmetric and asymmetric patterns becomes important. With $S_{f}$, one continues ${ }^{3}$ as explained in $\$ 6.3 .3$ by setting up a system of equations using the excitation found through linear synthesis. Without the symmetry, the roots found by Orchard-Elliott synthesis first need to be balanced as explained next.

\subsubsection{Root BALANCING}

It is assumed that synthesis has been completed up to the point where the roots need to be converted by expanding eq. 6.32. Let these roots be represented in terms of Orchard-Elliott synthesis

$$
w_{n}=e^{a_{n}+j b_{n}}
$$

where $a_{n} \neq 0$ for the $N_{1}$ roots that lay in region I. In chapter 5 , extensive use was made of the feature that for the power pattern it does not matter whether $a_{n}$ is positive or negative. Particularly the dynamic range of the excitations was optimized by choosing the set of roots such that $\max \left\{\left|I_{n}\right|\right\} / \min \left\{\left|I_{n}\right|\right\}$ is minimal. When excitations corresponding to the default $N_{1}$ positions outside the circle (figure 6.18) are used in the collapsed distribution principle, the result will be a pattern with deep nulls inside the shaped region. Figure 6.19 illustrates this by showing several $\phi$-cuts of $S_{r x}$. Clearly this pattern is unacceptable for tracking purposes. Expansion of eq. 6.32, and solving of eq. 6.25 is fast. When the designer tries different root combinations exhaustively, it is very likely that a much better result will be found using a different set of roots. For example the set presented in figure 6.18. By moving two out of four roots outside the circle to their opposing angular reciprocal position, the response shown in figure 6.20 is obtained. It can be seen that the same $\phi$-cuts of $S_{r x}$ are now much smoother and thus better suited for tracking. This strategy has been applied

\footnotetext{
${ }^{3}$ Unless problems are encountered during the conversion from Schelkunoff roots to electrical excitations, in which case one must first follow $\$ 6.4 .5$.
} 


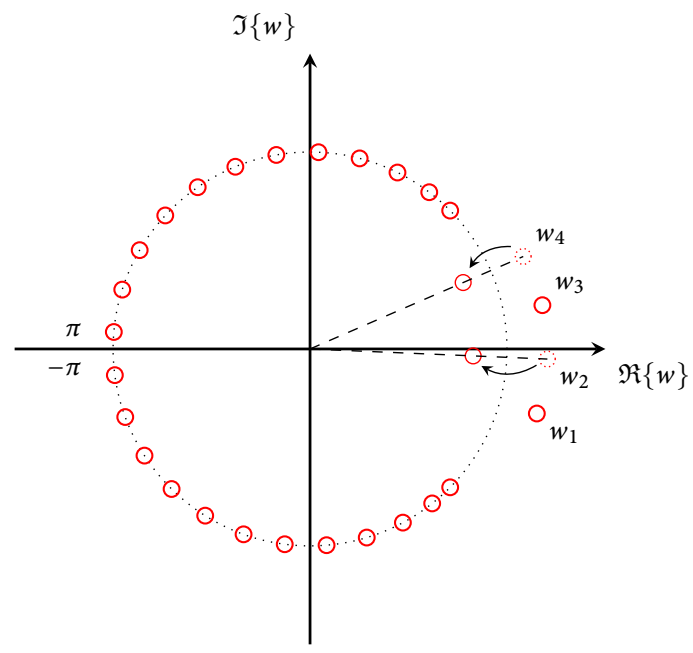

FIGURE 6.18 - Balancing the root positions for $S_{r x}(0, \theta)$

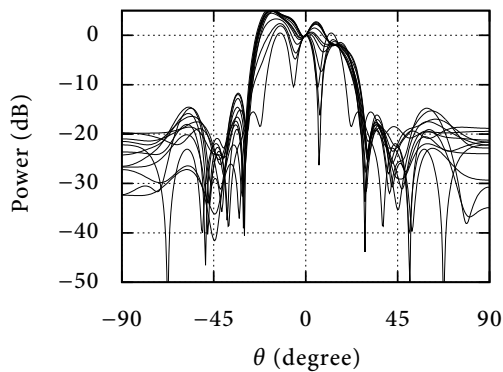

FIGURE 6.19 - Response after spreading unbalanced roots $\left(\forall n \in\{1 \ldots 4\} \mid a_{n}>0\right)$

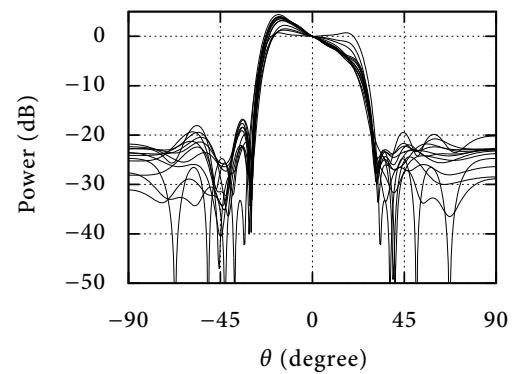

FIGURE 6.20 - Response after spreading balanced roots $\left(\forall n \in\{1,3\} \mid a_{n}>0\right)$

to the collapsed distributions of various different pattern shapes, and it is observed that a balanced set of roots always produces the best result in terms of ripple. Such a balanced set has $\left\lfloor N_{1} / 2\right\rfloor$ roots outside the circle and $\left[N_{1} / 2\right\rceil$ roots inside the circle, or vice versa.

In this example one could also have chosen to move $w_{1}$ and $w_{2}$ instead of $w_{2}$ and $w_{4}$ (figure 6.18). The resulting pattern then exhibits a slightly larger ripple, but the dynamic range of the excitations is also lower for this combination. A compromise can thus be made here.

Note-the above is based purely on the evidence obtained by synthesizing different pattern shapes. No proof is given that the balanced roots condition is sufficient for every possible pattern. 


\subsubsection{COMMON EXCITATION NORMALIZATION}

The response of the collapsed distribution of a non-symmetric pattern needs to be normalized in an explicit manner. To understand this, reconsider the (symmetric) example from \$6.3.4. Since the electrical excitations of an antenna array are always relative to each other, it is customary to express them relative to the value 1 . In the example, this value was given to the center $\left(\lceil L / 2\rceil^{\text {th }}\right)$ elements of the collapsed distributions. This means for every other $l^{\text {th }}$ element that its unnormalized excitation $\stackrel{\circ}{I}_{l}$ is given the value

$$
I_{l}=\frac{\stackrel{\circ}{I}_{l}}{I_{\lceil L / 2\rceil}}
$$

When the same is done for the planar array's $I_{m n}$ excitations

$$
I_{m n}=\frac{\stackrel{\circ}{I}_{m n}}{I_{\lceil M / 2\rceil\lceil N / 2\rceil}}
$$

it holds that

$$
I_{\lceil L / 2\rceil}=I_{\lceil M / 2\rceil\lceil N / 2\rceil}=1
$$

All excitations participating in the $\mathbf{A x}=\mathbf{b}$ system are then normalized to one common value. This automatically corrects any differences between the power of the individual collapsed distribution responses, under the condition that they are symmetric over the center.

Note-whenever eq. 6.36 is allowed (viz., symmetry exists), rewriting the system of equations as in the example improves its robustness. Therefore, let $\hat{\mathbf{A}}$ and $\mathbf{b}$ denote the coefficients that correspond to one particular collapsed distribution included in the system. Every row-element combination $\hat{\mathbf{A}}_{l} \mathbf{x}^{\mathrm{T}}=\hat{b}_{l}$ can be written as $\hat{\mathbf{A}}_{l} x^{\mathrm{T}}=$ $\hat{b}_{l}\left(\hat{\mathbf{A}}_{[L / 2]}\right)$ due to eq. 6.36. In other words, it can be expressed proportionally to the collapsed distribution's center element excitation. The case where $l=\lceil L / 2\rceil$ becomes trivial, so it can be left out. For the other equations expanding $\hat{b}_{l}\left(\hat{\mathbf{A}}_{\lceil L / 2\rceil}\right)$ and rearranging it so that all $\hat{\mathbf{A}}$ terms are on the left hand side, gives a more densely populated matrix A. Usually this form produces better results.

Without quadrantal symmetry, the center excitation values are no longer a valid normalization point. Any collapsed distribution response is guaranteed to intersect at the zenith, hence this is always a valid place for normalization. The excitations are therefore adjusted by

$$
I_{l}=\frac{\left|\stackrel{\circ}{I}_{l}\right|}{\left|F_{\phi_{c}}\left(0^{\circ}\right)\right|} e^{j<I_{l}}
$$




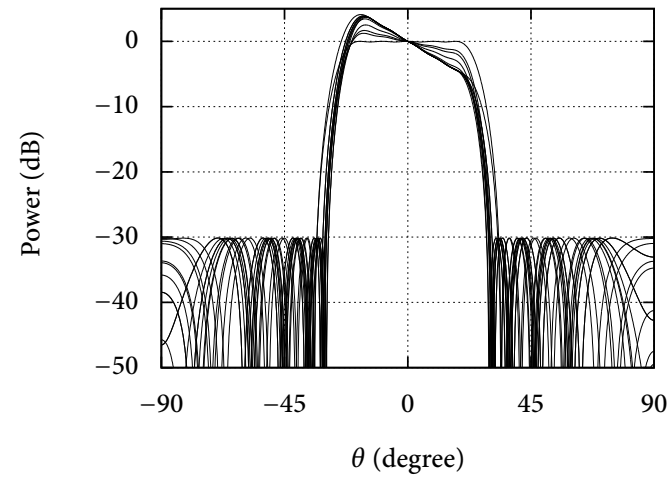

FIgURE 6.21 - Collapsed $S_{r x}$ responses with their center normalized to $0 \mathrm{~dB}$

such that $20 \log _{10}\left|F_{\phi_{c}}\left(0^{\circ}\right)\right|=0 \mathrm{~dB}$, as seen in figure 6.21. For this reason the $S_{r x}$ and $S_{r y}$ definitions placed the $0 \mathrm{~dB}$ point at $\theta=0^{\circ}$ rather than their peak value. Often this power normalization is sufficient for most practical purposes. However, when the lowest possible ripple is pursued or more complicated shapes desired (e.g., a planar equivalent of the $\csc ^{2}$ shape [91]), synthesis should also be performed relative to the MRA

\section{$M R A$ relative shaping}

In Orchard-Elliott synthesis [91], the shape function $S$ is defined relative to $\psi_{o}$ such that $G\left(\psi_{0}\right)=\max \{G\}$ (i.e., the peak of the main beam). A constant $C_{1}$ normalizes $G\left(\psi_{0}\right)$ to $0 \mathrm{~dB}$. To define $S$ in such a way is not ideal when $G(\psi)$ is asymmetric. After being normalized by eq. $6.37, G\left(\psi_{0}\right)$ is not necessarily $0 \mathrm{~dB}$. The most notable consequence is that the sidelobes will not arrive at the specified power level. However, the shaped region is also affected to some extent. Without symmetry, the correct way of specifying $S$ is relative to the MRA.

To specify $S$ relative to the MRA, say $\psi_{m}$, this location needs to be known. Unfortunately it is not possible to predict exactly where $\psi_{m}$ will be, because the shape of $G$ changes as it converges on $S$. A good estimate can be found by making use of the fact that the shaped region stays confined between two particular roots. Let the pattern be organized as in chapter 3 and [91]. The first $N_{1}$ roots of $G$ are then positioned in the shaped region, confining the latter between $-\pi$ and $b_{N_{1}+1}$. Consequently $\psi_{m} \simeq \frac{b_{N_{1}+1}-\pi}{2}$, as identified in figure 6.22. The balance between the left and right transient regions (figure 3.25) determines the accuracy of the estimate.

Recall from chapter 3 that [91] associates a constant $C_{2}$ with $S$, to compensate for small errors made in the normalization of $G\left(\psi_{o}\right)$. Since $S$ is now defined relative to $\psi_{m}$, a constant $C_{2}$ should be computed such that $G\left(\psi_{m}\right)$ goes to $0 \mathrm{~dB}$. If $G\left(\psi_{m}\right)$ 


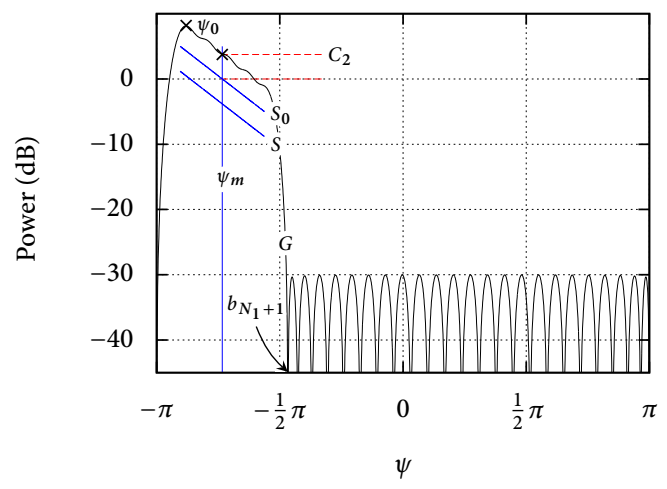

FIGURE 6.22 - Shaping $G\left(4^{\text {th }}\right.$ iteration $)$ to $S_{r x}\left(0^{\circ}, \theta\right)$ relative to the MRA instead of $\psi_{0}$. Note the use of $\psi$-space (chapter 3 ).

were to end up at e.g. $0.5 \mathrm{~dB}$, then $G$ must be shaped to $S-0.5$ in the next iteration. In figure 6.22 this has been illustrated as $S=S_{0}-C_{2}$.

\subsubsection{EXPANDING LARGE POLYNOMIALS}

Finally, before presenting the SHA-169 synthesis results, the size of the collapsed distributions is considered. For many of the entries in table 6.3, straightforwardly multiplying out the right hand side of eq. 6.32 can no longer be done in reasonable time. Let the roots of eq. 6.32 be collected in a vector $\mathbf{w}$. The coefficients $\mathbf{c}$ of the polynomial (i.e., excitations) can be found much faster with the 'Summation Algorithm' (i.e., poly(w) in Matlab) instead. In spite of relatively good numerical

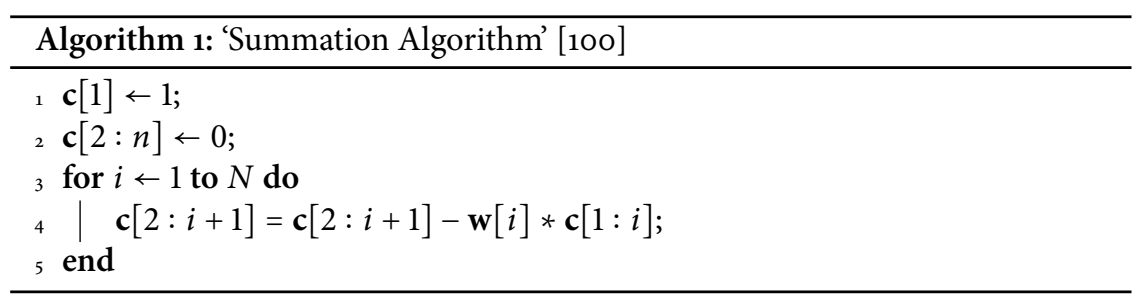

stability, this algorithm does suffer from rounding errors [100]. Results may vary depending on the set of roots, but on several occasions where $N \gtrsim 57$, standard IEEE-754 double precision floating-point arithmetic was found to be inadequate. Figure 6.23 shows the conversion of a flat-top pattern for $N=71$ (i.e., $\phi_{c}=16.01^{\circ}$ ), to illustrate what these errors can lead to. Such a response is not suitable for further use. 
An obvious solution to this problem is to use higher precision arithmetic. All the collapsed distributions discussed in this work were indeed expanded using quad precision arithmetic, available in the Advanpix arbitrary precision toolbox for Matlab [1]. However, there exists a solution that does not require special tools as well. Wilkinson's polynomial showed that in particular the coefficients in the center of the polynomial are affected by rounding errors [131]. Furthermore, the order in which the roots in $\mathbf{w}$ occur does not affect the resulting coefficients $\mathbf{c}$. It does determine which coefficients are going to be affected the most by the rounding errors. Since large collapsed distributions often produce a spacing $d_{x^{\prime}}<0.5 \lambda$, their visible region is smaller than the physical visible region (i.e., $-90^{\circ} \ldots 90^{\circ}$ ). There is thus a good probability that one can find an $\iota$ so that the differently ordered roots

$$
\mathbf{w}(i)=\stackrel{\circ}{\mathbf{w}}((i+\iota) \bmod (N-1))
$$

converted by algorithm 1 yield excitations for which only the parts of the pattern outside the visible region are affected by errors. These excitations can be used in the system of equations. Should the visible region not be smaller than $180^{\circ}$, then it is still possible to exploit the fact that the excitation furthest away from the center affect the pattern's shape the least [74].

\subsubsection{Results}

If the aforementioned is accurately applied to the collapsed distributions of $S_{f}$, $S_{r x}$ and $S_{r y}$, the SHA-169 array will give patterns as seen in figures 6.24 to 6.26. Each pattern features a smoothly shaped region, albeit with hexagonal boundaries. The performance measures that were used for the SRA (table 6.1) have also been compiled for the SHA in table 6.4. This shows that the use of hexagonal arrays

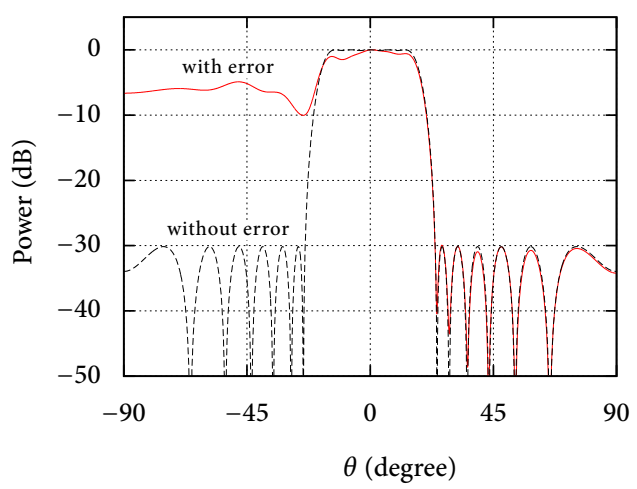

FIGURE 6.23 - Rounding errors made during root to excitation conversion 


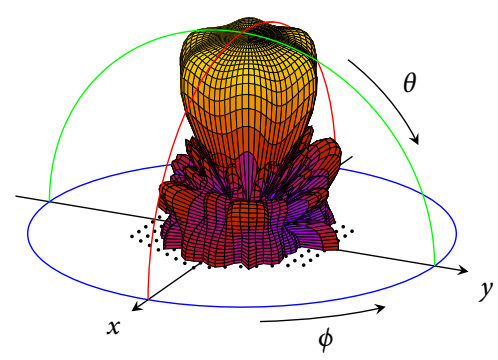

FIGURE 6.24 - Hexagonal $S_{f}$ pattern

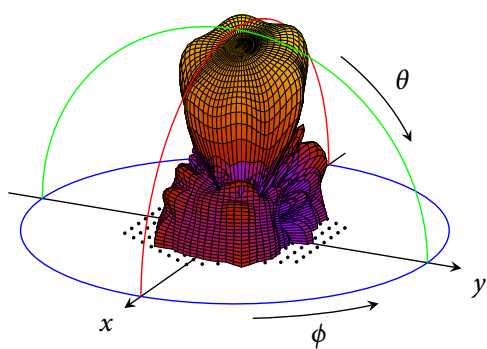

FIgURE 6.25 - Hexagonal $S_{r x}$ pattern

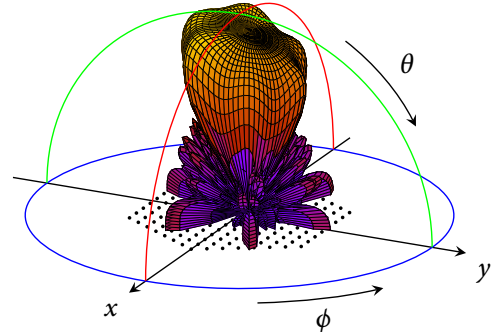

FIgURE 6.26 - Hexagonal $S_{r y}$ pattern

does sacrifice some performance. Most notably in the sidelobe levels, which are (according to the expectations [4]) higher than those of the rectangular array. Lower sidelobes can be obtained at the cost of beamwidth, by setting a lower SLL during linear synthesis. As a consequence of increased directivity, the wider beam could be compensated by placing the elements $\lambda / \sqrt{(3)}$ apart instead of $\lambda / 2$ (\$6.2.2). In terms of ripple, the performance has also decreased compared to rectangular arrays. However, all three shapes are well within the tolerance of shaped-pattern tracking (chapter 4). The implications for DoA estimation will be more significant (\$4.3.2), although the increased ripple can partially be explained by the fact that the $\phi$-cuts yield a curve that is sometimes concave or convex, similar to the effect discussed in $\$ 5.3 .2$.

TABLE 6.4 - SHA shaped pattern performance

\begin{tabular}{ccccc} 
Shape & Max. Ripple & Avg. Ripple & Max. SLL & Avg. SLL \\
\hline$S_{f}$ & $0.58 \mathrm{~dB}$ & $0.08 \mathrm{~dB}$ & $-21.9 \mathrm{~dB}$ & $-29.3 \mathrm{~dB}$ \\
$S_{r x}$ & $0.68 \mathrm{~dB}$ & $0.12 \mathrm{~dB}$ & $-20.9 \mathrm{~dB}$ & $-27.9 \mathrm{~dB}$ \\
$S_{r y}$ & $0.57 \mathrm{~dB}$ & $0.12 \mathrm{~dB}$ & $-23.4 \mathrm{~dB}$ & $-31.9 \mathrm{~dB}$
\end{tabular}




\subsection{STEERING AND SHAPE PRESERVATION}

Having established the three 'basic' beampatterns, the questions that remain are how they will behave under different steering angles and whether they are indeed suitable for tracking purposes. It shall first be demonstrated that, because of its higher degree of rotational symmetry, the hexagonal array is much more capable at retaining the shape of the patterns when steered.

\subsubsection{STEERING}

Recall that the orientation of the beam can be changed 'lossless' to any of the pseudo axes, simply by mapping the excitations differently to the element positions. Such a mapping is easily realized when the excitations are organized in a matrix $\mathbf{W}$, with indexing as explained in \$6.2.2. Let every $m^{\text {th }}$ row where $m<\lceil M / 2\rceil$ be padded with leading zeros and every $m^{\text {th }}$ row where $m>\lceil M / 2\rceil$ with trailing zeros (i.e., dummy values). The transpose $\mathbf{W}^{T}$ will rotate the patterns' shape by $300^{\circ}$ (and flip it over the $x$-axis), as illustrated by the small example below:
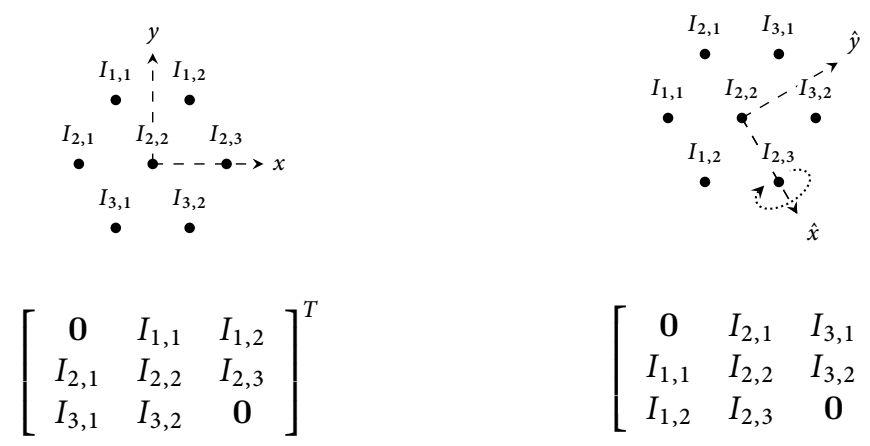

FIGURE 6.27 - Pattern rotation by excitation mapping

Rotation to any of the other pseudo axes is simply derived by mirror operations. This mapping greatly reduces the need for $\phi$ steering outside the pattern's axes of reference; thus keeping the angular coverage more consistent. To change the elevation angle, and make the last small changes in $\phi$, every $m n^{\text {th }}$ excitation still has to be weighed according to expression 6.6. Yet, given their coverage, the patterns shown here will not need to be steered further than $10^{\circ}$ away from the pseudo axes. This makes $\phi=50^{\circ}$ one of the larger steering angles that will be required. Figures 6.28 and 6.29 show that the shape of the pattern is very similar between $\phi=0^{\circ}$ and $\phi=50^{\circ}$. Although the hexagonal shape may not provide the ideal coverage area, this consistency will make tracking much easier. 


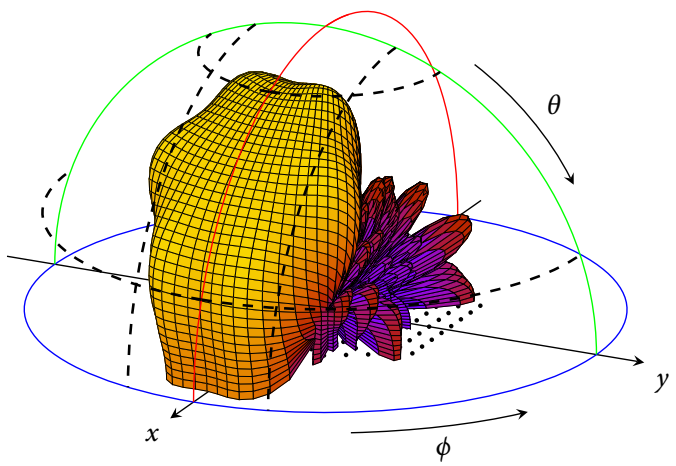

FIGURE 6.28 - Coverage after steering the SHA-169 $S_{f}$ pattern to $\left(0^{\circ}, 35^{\circ}\right)$

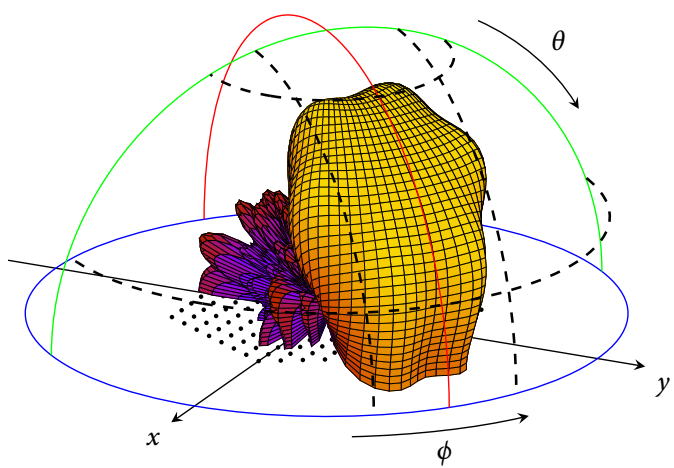

FIGURE 6.29 - Coverage after steering the SHA-169 $S_{f}$ pattern to $\left(50^{\circ}, 35^{\circ}\right)$

\section{Limitations}

In spite of a consistent angular coverage, the steering angle is still limited. If the aliasing criterion (\$6.2.2) is applied, the maximum elevation angle is most limited when $\phi_{d}=30^{\circ}$ or rotationally equivalent. For the $1 / 2 \lambda$ spaced SHA, $\theta_{d}$ cannot exceed $55^{\circ}$ if aliasing of the shaped region is not allowed. However, it should be noted that when $\theta_{d}=45^{\circ}$, the angular region already reaches up to $\theta=90^{\circ}$. Should the larger $\sqrt{3} / \lambda$ spacing from $\$ 6.2 .2$ be needed (e.g., when mutual coupling becomes a problem), then the steering angle will be limited to $35^{\circ}$.

\subsubsection{TRACKING COMPATIBILITY}

To assess whether the patterns remain sufficiently consistent for tracking while being steered, the combinations $S_{f}$ with $S_{r x}$, and $S_{f}$ with $S_{r y}$ are steered to $50^{\circ}$ azimuth and $45^{\circ}$ elevation. In terms of shape retention, this steering angle is repre- 
sentative for the worst case needed in covering the entire upper hemisphere with respect to the array center. From the azimuth- and elevation planes respectively, crosscuts are provided in figure 6.30 and figure 6.31. Figure 6.30 depicts $S_{r x}$ as being flat in the plane parallel to the page and ramp-shaped perpendicular to the page, while figure 6.31 shows $S_{r y}$ in the same manner. Herein it can be seen that the power differences between the flat and ramp shaped patterns are quite consistent within a $20^{\circ}$ by $30^{\circ}$ coverage area, both in the $\phi$ and $\theta$ direction. Based on this observation, the ideas from chapter 4 can directly be carried over to the 2-D domain. The steering angle can simply be updated based on the power levels associated with the boundaries of the $20^{\circ}$ by $30^{\circ}$ coverage area.

Note-It can now also been seen why it would be convenient to reverse the ramp if the zenith is crossed (as announced by $\$ 4.2 .2$ ). By doing so, the direction of the slope remains invariant for all azimuth angles. This ensures that there will be no awkward transitions of the slope's direction when the azimuth steering angle is updated.

\subsection{FUTURE WORK}

As the presented extension to planar arrays is largely based on techniques for linear arrays, most of the material covered by the preceding chapters is still applicable. In addition, it has been shown that the basic concept of shaped-pattern tracking, as explained for linear arrays in chapter 4, can be utilized for hexagonal planar arrays in two orthogonal directions. However, there are various aspects of planar array use that still require further research.

Firstly, more detailed definitions needs to be given for tracking. These should primarily encompass an efficient distribution of pattern triplets-which covers all the azimuth elevation space needed for a certain room size-and rules for updating the steering angle. The latter should for example define safety margins as discussed in $\$ 4.2 .2$ and the direction of the slope as noted above. Thereafter, the proposed tracking principle can initially be analyzed by means of simulations similar to those explained for the linear case in chapter 4 .

Secondly, it should be investigated how the element factor compensation presented in chapter 5 works in conjunction with the principle of collapsed distributions. Given that the basic synthesis principles remain unchanged, it should be possible to compensate the spherical element factor. For each of the collapsed distribution responses, a corresponding linear element factor can be found by taking an azimuth cut at $\phi=\phi_{c}$. Such a cut, taken from the spherical gain of figure 4.4 , has been depicted in figure 6.32. The response at this angle would correspond with the collapsed distribution response visualized in figure 6.12. It is not known whether the mutual coupling effect can be compensated in the same way. At least for the linear techniques reported in chapter 5 there is not a straightforward extension for collapsed distributions. 


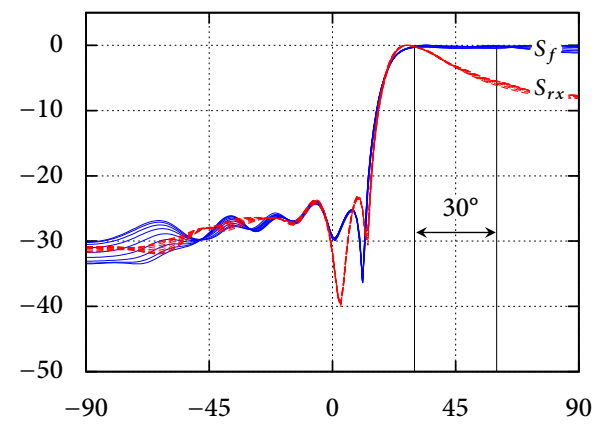

Figure 6.30 - Azimuth crosscuts of the $S_{f}$ and $S_{r x}$ patterns steered to $\left(50^{\circ}, 45^{\circ}\right)$, covering the range $40^{\circ} \leq \phi \leq 60^{\circ}$ in the azimuth plane (20 in figure 6.31)

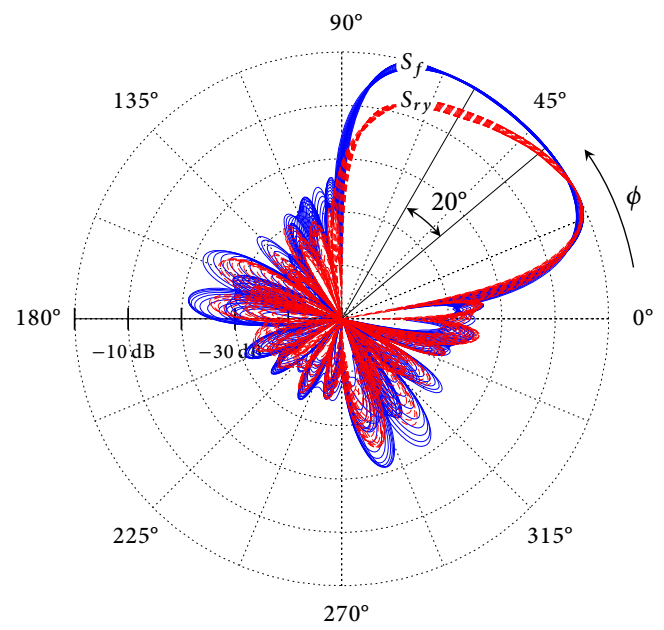

FIGURE 6.31 - Elevation crosscuts of the $S_{f}$ and $S_{r y}$ patterns, steered to $\left(50^{\circ}, 45^{\circ}\right)$ and covering the elevation range $30^{\circ} \leq \theta \leq 60^{\circ}\left(30^{\circ}\right.$ in figure 6.30) 


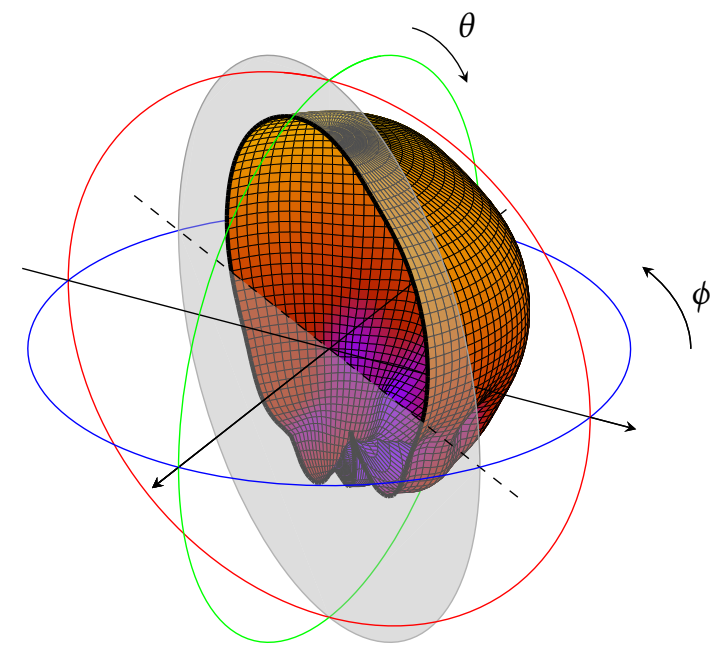

FIgURE 6.32 - Spherical element $\theta$-cut response

Furthermore, although every example encountered thus far suggests it, it has not been proven that the balanced roots condition is sufficient for asymmetric beampatterns in general. It will be beneficial for the procedure if proof can be found, or alternatively, the reason why unbalanced roots do not work well.

Finally it may also be interesting to investigate how far the array size can be scaled down. Even though the SHA already requires much less elements than the SRA, 169 antennas is still a substantial amount.

\subsection{Conclusions}

Shaped-pattern tracking has been extended to planar antenna arrays for increased directionality. This was achieved by applying the linear tracking algorithm from chapter 4 in two orthogonal directions, such that both the $\phi$ and $\theta$ component of the required steering angle can be determined. A triplet of differently shaped patterns needs to be synthesized for this approach. By utilizing a rectangular grid array, synthesizing these patterns is very straightforward by means of separable weightings. However, patterns that are obtained this way do not retain their shape well when steered. The use of (standard) hexagonal arrays, which exhibit a high degree of rotational symmetry, is therefore recommended.

Synthesis of low-ripple tracking beampatterns has been made possible for the standard hexagonal array by employing the principle of collapsed distributions. In this procedure a particular azimuth angle $\phi_{c}$ is chosen and in that direction the planar array element positions are projected onto an equivalent linear array. Subsequently, the Orchard-Elliott method from chapter 3 can be invoked to shape the pattern of 
this array. After having performed this operation for a selection of azimuth angles, a system of equations is obtained in which the unknown planar excitations values are related to the known (synthesized) linear values. Solving this system produces a pattern that is constrained by the shapes that were synthesized for the individual $\phi_{c}$ collapsed distributions. Previously this method was only applicable to beampatterns that are quadrant symmetric. This chapter showed how it can also be used for patterns which do not satisfy this property.

In order to apply this method to asymmetric shapes, it has been found that two additional measures must be taken. Firstly, it was empirically determined that there must exist a balance between the number of roots inside and outside the unit circle. Secondly, the excitations of the collapsed distributions need to be normalized explicitly to a common value. The array's response at the zenith was chosen for this purpose.

The resulting patterns feature (on average) a ripple of $0.6 \mathrm{~dB}$, which is well within the bounds for shaped-pattern tracking. Moreover, due to the hexagonal array's rotational symmetry, consistency of the shape of the beampatterns is improved compared to beampatterns produced by rectangular arrays when steered. Although a detailed plan for tracking has not yet been given, this consistency suggest that the ideas presented in chapter 4 can directly be applied to two orthogonal planes in full $(\phi, \theta)$-space. In addition, because collapsed distributions are linear arrays, most of the properties and optimizations discussed in the previous chapters can still be exploited. 



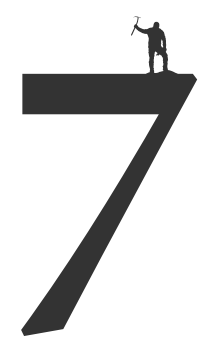

\section{CONCLUSIONS AND RECOMMENDATIONS}

\footnotetext{
o meet the growing need for capacity in short-range wireless communications, migration to the $60 \mathrm{GHz}$ frequency band is considered to be a nice option. At this frequency, antennas must feature high gain and directionality. Antenna arrays, or more specifically beamformers, suit these requirements well. Especially if use is made of analog beamforming, wireless communication by directional $60 \mathrm{GHz}$ beams will deliver ample bandwidth while remaining cost- and energyefficient. The catch of highly directional transmission/reception is that the direction of the beams must be managed adaptively in the presence of mobile wireless devices. The position of such devices needs to be tracked in order to steer the beam in that direction. In particular when a beamformer is all-analog, this poses a significant challenge. To cope with tracking using all-analog beamforming, this thesis has presented a method based on shaped beampatterns.
}

Shaped beampatterns are obtained via synthesis, which implies finding the complex array excitation values (i.e., phase and amplitude) that give rise to the shape that is desired. When this shape is used for tracking purposes, it may exhibit only limited ripple (i.e., deviations from the specification). In chapter 3 it was shown that Orchard-Elliott synthesis is good at shaping the beampattern of equispaced linear arrays with a constrained ripple. To establish a unique relation between a given angle and received power, an asymmetric (ramp shaped) beam with uniform sidelobe level was defined and synthesized using this method. Such a pattern enables direction-of-arrival estimation based solely on measuring the received power.

It was also shown that an absolute measure of power does not suffice for correct DoA estimation, due to the influences of e.g. path loss. To tackle this problem, a tracking mechanism which alternates between two different patterns has been proposed in chapter 4 . The second shape, a flat-topped beam, features a power response that is independent of the angle. As such, the measured power difference can be used for DoA estimation, and subsequently for tracking. 
Among other simplifications, Orchard-Elliott synthesis assumes that arrays consist of perfect isotropic elements. In practice the element factor is not isotropic, but typically exhibits a decaying gain towards endfire. If this is the case, the shape of the beam deforms when steered. The deviations from the specification are too large for the proposed tracking algorithm. A modification of the Orchard-Elliott method was presented in chapter 5 , to compensate the (measured) non-isotropic gain of a realistic element factor. Furthermore, excitations become a combination of delay and amplification when realized. It has been investigated whether the required values are suitable for realization. If the appropriate optimization steps are taken after synthesis, this is indeed the case.

Finally, as linear arrays only enhance directionality in one dimension, the use of planar arrays was studied in chapter 6 . It has been found that the conventional rectangular array is not able to sufficiently preserve the shape of the beam under large steering angles. A hexagonal array structure performs better in that respect. To synthesize low-ripple asymmetric beampatterns for this structure, an adapted procedure that uses the principle of collapsed distributions is employed. Since this is a logical extension of Orchard-Elliott synthesis, most of the principles that were discussed for linear arrays can also be applied to the planar array. Tracking is achieved by performing the one-dimensional mechanism in two orthogonal directions.

\subsection{CONTRIBUtions}

Principally with analog adaptive beamforming in mind, but also in a broader sense, the main contributions of this thesis are as follows:

»A shaped-pattern based tracking algorithm (for linear antenna arrays) that only requires two power measurements from different patterns to function. This algorithm works regardless of the underlying type of beamforming.

»Definitions of suitable shaped beampatterns and the synthesis thereof. A combination of ramp- and flat-topped beampatterns, synthesized using the Orchard-Elliott procedure, has been verified to work by means of Monte Carlo simulation.

"Element factor aware Orchard-Elliott synthesis that is able to compensate the effects of non-isotropic antenna gain, by adjusting the array excitations accordingly.

"Verification of excitation values for realizability, in terms of the required amplification range and delays. The amplification range will require an additional optimization step that involves displacing polynomial root positions, but afterwards all excitation values are within realistic bounds.

"Support for hexagonally shaped planar antenna arrays, to enhance directionality. Synthesis of tracking patterns can be achieved with an adapted form of collapsed distributions, which was previously only applicable to quadrant symmetric beampatterns. Tracking is realized by applying the algorithm for linear arrays in two orthogonal directions. 


\subsection{RECOMMENDATIONS}

As stated in the introduction chapter, the objective of this thesis was to develop a tracking algorithm for $60 \mathrm{GHz}$ wireless communication, that can cope with the limited flexibility of an all-analog beamformer. The solution that has been proposed is an algorithm that makes use of alternating ramp- and flat-shaped beampatterns. This thesis has covered part of the approach, there are still many opportunities for further research. The recommended directions are given below.

A ramp-shaped pattern was chosen for DoA estimation because it gives the most straightforward power-to-angle relation. Further research could include a study of different asymmetrical shapes to identify their (dis)advantages.

For communication capabilities, more research is needed at system level. The tracking algorithm presented in this thesis only concerns situations where the mobile devices stays within reach of a single array, and LoS connection is possible. These conditions are not guaranteed. Investigated solutions to prevent connection loss are for example: hand-off to another nearby antenna array [20]; relay techniques [89]; or proceeding via reflections [53]. In particular for SOWICI it has been investigated how sensors available in the mobile devices [38], and the established $2.4 \mathrm{GHz}$ equipment [32], can be used to assist in tracking with multiple arrays. Although the use of shaped patterns does not directly pose a problem for either of these approaches, it may be worthwhile to investigate their collaboration opportunities. Somewhat related to these topics is the fact that chapter 4 also assumed that the mobile devices transmit omnidirectional. If beamforming is performed on the receiver and the transmitter side, the (shaped-pattern) tracking mechanism must be able to account for this. Maintaining alignment between two beams, whether or not based on their shape, will be a substantial research topic. Furthermore, the simulations presented in chapter 4 could be extended to include the effects of interferers and more channel characteristics such as scattering and fading. Since compatibility with either of the IEEE $802.15 .3 \mathrm{C}[67$ ] or $802.11 \mathrm{AD}$ [68] standards is desirable, their requirements could also be included in the simulations. The planar array case additionally requires a detailed plan for updating steering angles.

Finally, to quantify the advantages of using shaped beampatterns for tracking, and to make a final assessment of its feasibility, a prototype is needed. Remotely controlled optical beam steering has already been demonstrated in SOWICI using a small prototype [28]. Modifications would be required, but this prototype could be used as a starting point to induce the proposed beams optically, and measure their shapes. If measurements show that the actual beampattern is not properly shaped, mutual coupling should be considered first. For linear arrays, existing techniques were discussed in chapter 5 , for planar arrays a direct solution could not be given. Improvement for planar arrays may be possible by employing optimization techniques as a final step after spreading the collapsed distributions. Although global optimization did not perform well as a stand-alone solution, it could prove useful after seeding a good starting point obtained by collapsed distributions [13]. 



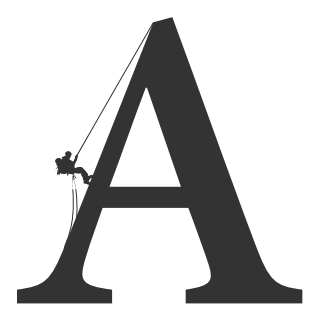

\section{NumericAl DATA}

This appendix provides numerical data that may be helpful in reconstructing the synthesis examples from chapter 3. Tables A.1 and A.2 correspond respectively to the examples in \$3.2.3 and \$3.3.1.

TABLE A.1 STRUCTURE

The first column $(n / p)$ lists index numbers of variables presented the columns that follow; the $n$ subscript is related to roots, $p$ to peaks. For the initial iteration and starting point, both the null $\left(\dot{\psi}_{n}\right)$ and peak $\left(\dot{\psi}_{p}\right)$ locations are given, as well as the peak height $\left(F_{0}\left(w_{p}\right)\right)$ and the specified height $\left(F\left(w_{p}\right)\right)$. Note that specifications have been converted from decibels to absolute scale, to allow a direct comparison with $F_{0}$. For every other iteration, the root perturbations $\left(\delta \psi_{n}\right)$ are shown along with the details relating to peaks. In addition, the bottom row shows the initial value for normalization $\left(C_{0}\right)$ and the complex relative difference $(\delta C)$ needed in the subsequent iterations.

\section{TABLE A.2 STRUCTURE}

Each of the columns shown in this table corresponds to a vector that is used in the algorithm. From the starting pattern, the values of $\mathbf{f}$ define-in order-the four $a_{n}$ from region I, all $14 b_{n}$ from region I \& II (minus the fixed root) and the normalization constant $C$. The $\mathbf{g}$ values represent respectively the allowed maxima of the $G-S$ ripple, the allowed minima and the height of each sidelobe. For every intermediate iteration, the current extrema $(\hat{\mathbf{g}})$ and their locations in $\psi$-space $(\psi)$ are listed in the same sequence as $\mathbf{g}$. The order of the computed perturbations $\delta \mathbf{f}$ matches with $\mathbf{f}$. 


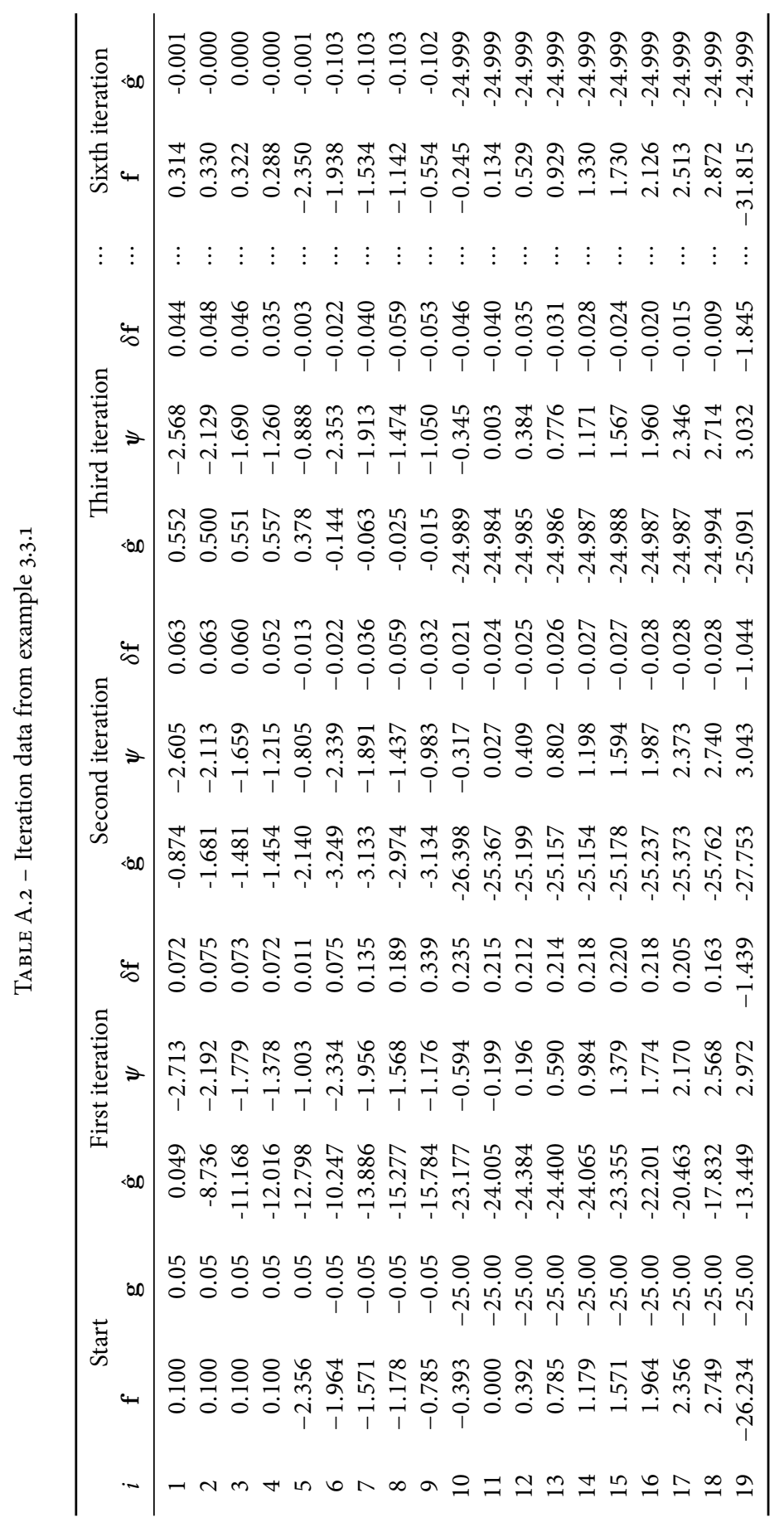





\section{ACRONYMS}

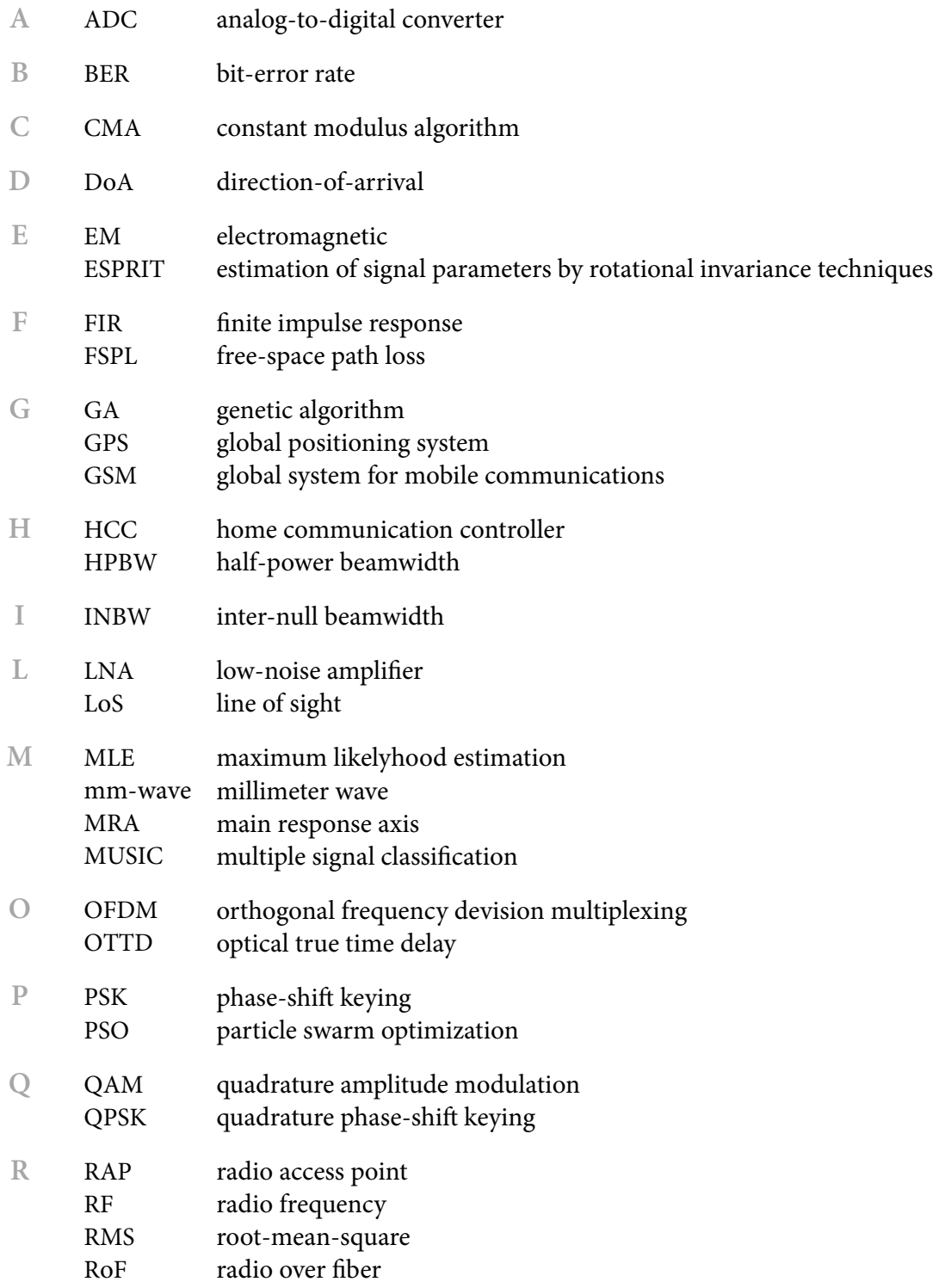


$S \quad$ SA simulated annealing

SES smart energy systems

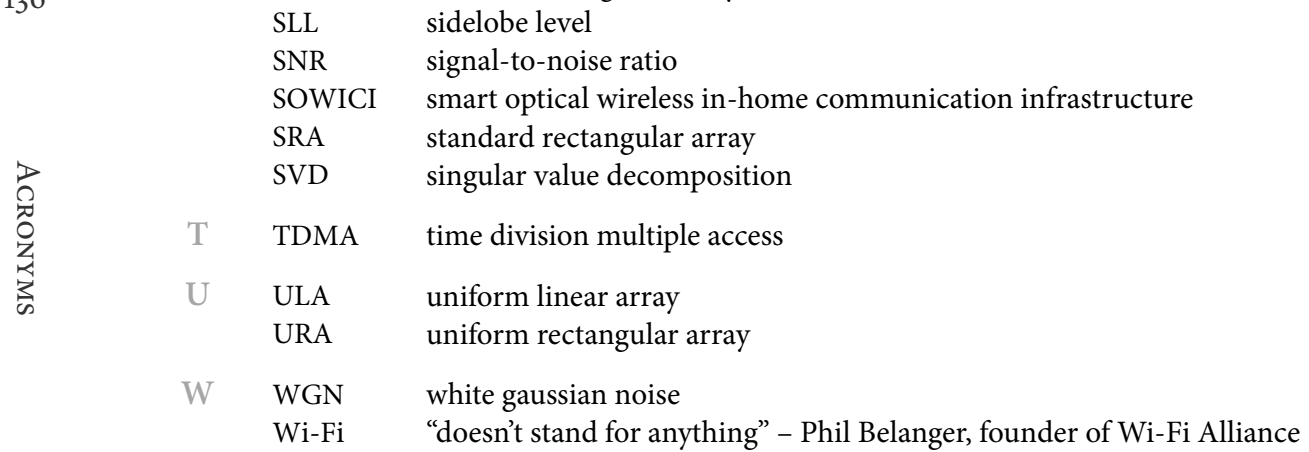




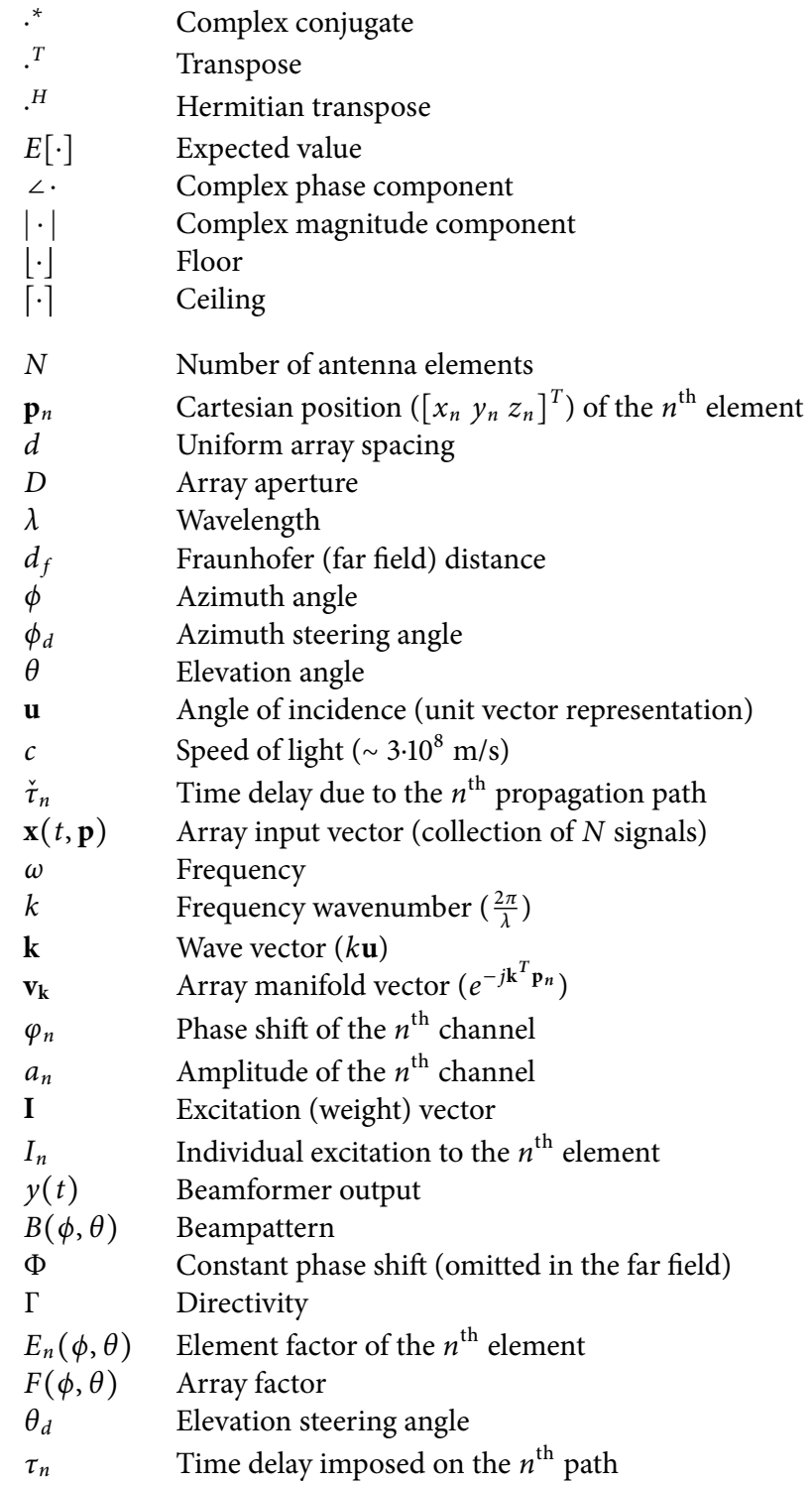




\begin{tabular}{|c|c|c|}
\hline \multirow{7}{*}{138} & $\mathbf{R}_{x x}^{-1}$ & Spatial covariance matrix \\
\hline & $\gamma_{r x}$ & Cross correlation vector (between $\mathbf{x}$ and reference signal $r$ ) \\
\hline & $\mathbf{I}_{\text {opt }}$ & Wiener-Hopf optimal excitations \\
\hline & K & Sample size \\
\hline & $P$ & Power \\
\hline & $J$ & CMA cost function \\
\hline & $\nabla$ & Gradient \\
\hline \multirow{40}{*}{ 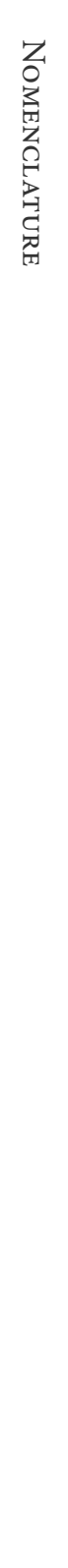 } & $\mu$ & CMA step size \\
\hline & $S(\theta)$ & Shape function \\
\hline & {$\left[\theta_{0}, \theta_{1}\right]$} & Shaped region \\
\hline & $F_{\theta}$ & Linear array factor in $\theta$-space \\
\hline & $\psi$ & Linearized elevation angle $\left(\frac{2 \pi d}{\lambda} \sin (\theta)\right)$ \\
\hline & $\bar{\psi}$ & Visible region in $\psi$-space \\
\hline & $F_{\psi}$ & Linear array factor in $\psi$-space \\
\hline & $w$ & Position on the unit circle $\left(e^{j \psi}\right)$ \\
\hline & $w_{n}$ & Polynomial root \\
\hline & $F(w)$ & Schelkunoff unit circle representation of the array factor \\
\hline & $\delta \psi$ & Collective root rotation \\
\hline & $\delta \psi_{n}$ & Angular root perturbation \\
\hline & $w_{p}$ & Local maximum (lobe peak) in $w$-space \\
\hline & $C$ & Power normalization constant for beampattern \\
\hline & $S_{r}$ & Ramp shape function \\
\hline & $G(\psi)$ & Orchard-Elliott synthesis - expression for array factor shaping \\
\hline & $a_{n}$ & Orchard-Elliott synthesis - root $\left(w_{n}\right)$ decomposition $\ln \left(\left|w_{n}\right|\right)$ \\
\hline & & Orchard-Elliott synthesis $-\operatorname{root}\left(w_{n}\right)$ decomposition $\angle w_{n}$ \\
\hline & $\delta a_{n} / \delta b_{n}$ & Orchard-Elliott synthesis - decomposed root perturbations \\
\hline & $\psi_{m}$ & Main lobe position (i.e., where $\max \{G \psi\}$ is situated) \\
\hline & f & Orchard-Elliott synthesis - current pattern shape parameters \\
\hline & g & Orchard-Elliott synthesis - desired pattern shape parameters \\
\hline & $\psi$ & Orchard-Elliott synthesis - current pattern extrema locations \\
\hline & $\hat{\mathbf{g}}$ & Orchard-Elliott synthesis - current pattern extrema values \\
\hline & A & Orchard-Elliott synthesis - Jacobian matrix \\
\hline & $\Delta \tilde{\theta}$ & Shaped region width $\left(\tilde{\theta}_{1}-\tilde{\theta}_{0}\right)$ \\
\hline & $E_{Q}$ & Global optimization synthesis - cost function \\
\hline & $h_{q}$ & Global optimization synthesis - weight function \\
\hline & $q$ & Global optimization synthesis - cost evaluation point \\
\hline & & Global optimization synthesis - relative error \\
\hline & $F_{\min } / F_{\min }$ & Global optimization synthesis - shaping masks \\
\hline & $\Delta P / \Delta \theta$ & Slope steepness ( $\mathrm{dB} /$ degree $)$ \\
\hline & {$\left[\tilde{\theta}_{0}, \tilde{\theta}_{1}\right]$} & Shaped region obtained after synthesis \\
\hline & $\mathcal{L}_{r}$ & Linear ramp shaped power pattern \\
\hline & $S_{f}$ & Flat-top shaping function \\
\hline & $\mathcal{L}_{f}$ & Linear flat-topped power pattern \\
\hline & $P_{f}$ & Flat-topped beam power response \\
\hline & $P_{r}$ & Ramp shaped beam power response \\
\hline & $P_{\max }$ & Maximum expected power response \\
\hline & $P_{\min }$ & Minimum expected power response \\
\hline
\end{tabular}




\begin{tabular}{|c|c|}
\hline$\theta_{r}$ & DoA relative to $\theta_{0}$ \\
\hline$\delta P$ & Relative power difference \\
\hline$\Upsilon$ & Tracking speed parameter $\left(\mathcal{L}_{f}\right.$ usage $/ \mathcal{L}_{r}$ usage $)$ \\
\hline$\tau_{p}$ & Sampling time \\
\hline $\mathbf{T}_{f}(i)$ & Flat-topped beampattern subjected to $i^{\text {th }}$ scan angle \\
\hline $\mathbf{T}_{r}(i)$ & Ramp shaped beampattern subjected to $i^{\text {th }}$ scan angle \\
\hline$\eta$ & Path loss exponent \\
\hline$f_{s}$ & Sampling frequency \\
\hline$E(\theta)$ & Identical element factors \\
\hline$G E(\psi)$ & Beampattern shaping expression including the element fact \\
\hline$\left[\psi_{0}, \psi_{1}\right]$ & Shaped region in $\psi$-space \\
\hline$S^{\prime}(\psi)$ & Shape function shifted in $\psi$-space \\
\hline$\psi_{m}$ & Beampattern maximum (main lobe peak) \\
\hline e & Measured element factor \\
\hline$E^{\prime}(\psi)$ & Wrapped element factor in $\psi$-space \\
\hline$\psi_{p}$ & Wrapped element factor discontinuity location \\
\hline$E_{l}(\psi)$ & Partial element factor left of $\psi_{p}$ \\
\hline$E_{r}(\psi)$ & Partial element factor right of $\psi_{p}$ \\
\hline$\psi_{r}$ & Erroneous multi-peak detection replacement \\
\hline$B_{G}(\theta)$ & Beampattern shaped without the element factor compensate \\
\hline$B_{E}(\theta)$ & Beampattern shaped with the element factor compensated \\
\hline$I_{\max }$ & Maximum excitation magnitude $\left(\max \left\{\left|I_{n}\right|\right\}\right)$ \\
\hline$I_{\min }$ & Minimum excitation magnitude $\left(\min \left\{\left|I_{n}\right|\right\}\right)$ \\
\hline$\omega_{c}$ & Center (carrier) frequency \\
\hline$\Delta \theta_{x} / \Delta \theta_{y}$ & Shaped region surface boundaries \\
\hline & Number of antenna elements in direction orthogonal to $N$ \\
\hline$x_{m n}$ & Planar array's $m n^{\text {th }}$ element $x$ position \\
\hline$y_{m n}$ & Planar array's $m n^{\text {th }}$ element $y$ position \\
\hline$d_{x}$ & Uniform element spacing in the $x$ direction \\
\hline$d_{y}$ & Uniform element spacing in the $y$ direction \\
\hline$I_{m n}$ & Planar excitation to the $m n^{\text {th }}$ element \\
\hline$S_{r x}$ & Spherical ramp shape definition in $x$ direction \\
\hline$S_{r y}$ & Spherical ramp shape definition in $y$ direction \\
\hline$\hat{x} / \hat{y}$ & Axes rotationally symmetric to $x / y$ \\
\hline$\phi_{c}$ & Collapsed distribution angle \\
\hline$\alpha$ & Planar rotation angle \\
\hline$x_{m n}^{\prime}$ & Element's $x$ position after rotation \\
\hline$y_{m n}^{\prime}$ & Element's $y$ position after rotation \\
\hline$x^{\prime}$ & Frame of reference's $x$-axis after collapsing \\
\hline$\theta_{x^{\prime}}$ & Elevation angle measure in the $x^{\prime} z$-plane \\
\hline$d_{x^{\prime}}$ & Collapsed distribution's uniform element spacing \\
\hline$L$ & Number of elements in collapsed distribution \\
\hline$I_{l}$ & Excitation to the collapsed distribution's $l^{\text {th }}$ element \\
\hline$\epsilon_{m n}$ & Contribution factor ( 0 or 1$)$ for $m n^{\text {th }}$ planar element \\
\hline $\mathbf{w}$ & Root vector \\
\hline W & Matrix representation of planar array excitations \\
\hline
\end{tabular}





\section{BibLIOGRAPHY}

[1] Advanpix. Multiprecision computing toolbox for matlab. http://www . advanpix . com. Accessed: 15-11-2015. (Cited on page 118).

[2] B. Agee, S. V. Schell, and W. A. Gardner. Spectral self-coherence restoral: a new approach to blind adaptive signal extraction using antenna arrays. Proceedings of the IEEE, 78(4):753-767, April 1990. ISSN 0018-9219. doi: 10.1109/5.54812. (Cited on page 24).

[3] A. Aghasi, H. Amindavar, E. L. Miller, and J. Rashed-Mohassel. Flat-top footprint pattern synthesis through the design of arbitrary planar-shaped apertures. IEEE Transactions on Antennas and Propagation, 58(8):2539-2552, August 2010. ISSN oo18926X. doi: 10.1109/TAP.2010.2050420. (Cited on pages 47 and 101).

[4] J. A. G. Akkermans. Planar beam-forming antenna array for $60 \mathrm{GHz}$ broadband communication. PhD thesis, Technische Universiteit Eindhoven, Eindhoven, March 2009. (Cited on pages 83 and 119).

[5] J. A. G. Akkermans and M. H. A. J. Herben. Planar beam-forming array for broadband communication in the $60 \mathrm{GHz}$ band. In European Conference on Antennas and Propagation, pages 1-6, November 2007. (Cited on page 99).

[6] J. A. G. Akkermans, M. C. van Beurden, and M. H. A. J. Herben. Design of a millimeter-wave balanced-fed aperture-coupled patch antenna. In European Conference on Antennas and Propagation (EuCAP), pages 1-6, November 2006. (Cited on page 18).

[7] B. Allen and M. Ghavami. Adaptive array systems: fundamentals and applications. Wiley-Blackwell, 1 edition, February 2005. ISBN 9780470861899. (Cited on pages 3 , $5,7,13,16,19,20,21,22,24,87$, and 88).

[8] J. G. Andrews, S. Buzzi, W. C, S. V. Hanly, A. Lozano, A. C. K. Soong, and J. C. Zhang. What will $5 \mathrm{G} \mathrm{Be}$ ? IEEE Journal on Selected Areas in Communications, 32(6):10651082, June 2014. ISSN 0733-8716. doi: 10.1109/JSAC.2014.2328098. (Cited on pages 1 and 2).

[9] D. H. Archer. Lens-fed multiple beam arrays. Microwave Journal, 27:171, September 1984. (Cited on page 21).

[10] F. J. Ares-Pena. A note on the limitations of Orchard's method. IEEE Antennas and Propagation Magazine, 44(1):109, February 2002. ISSN 1045-9243. doi: 10.1109/74.997924. (Cited on pages 74 and 83). 
[11] F. J. Ares-Pena, R. S. Elliott, and E. Moreno. Design of planar arrays to obtain efficient footprint patterns with an arbitrary footprint boundary. IEEE Transactions on Antennas and Propagation, 42(11):1509-1514, November 1994. ISSN 0018-926X. doi: 10.1109/8.362787. (Cited on pages 101, 107, and 111).

[12] F. J. Ares-Pena, S. R. Rengarajan, A. Vieiro, and E. Moreno. Extension of Orchard's pattern synthesis technique for overdetermined systems. In Antennas and Propagation Society International Symposium, 1996. AP-S. Digest, volume 4, pages 1834-1837, June 1995. doi: 10.1109/APS.1995.530943. (Cited on page 48).

[13] F. J. Ares-Pena, J. A. Rodriguez-Gonzalez, and A. Vieiro. Efficient footprint patterns obtained by spreading out collapsed distributions. In Antennas and Propagation Society International Symposium, volume 1, pages 498-501, July 1997. doi: 10.1109/APS.1997.630207. (Cited on pages 101 and 129).

[14] F. J. Ares-Pena, J. A. Rodriguez-Gonzalez, E. Villanueva-Lopez, and S. R. Rengarajan. Genetic algorithms in the design and optimization of antenna array patterns. IEEE Transactions on Antennas and Propagation, 47(3):506-510, March 1999. ISSN oo18926X. doi: 10.1109/8.768786. (Cited on pages 51 and 85).

[15] V. Bakker. Triana: a control strategy for smart grids: forecasting, planning \& real-time control. PhD thesis, Universiteit Twente, Enschede, January 2012. (Cited on page 5).

[16] J. Barnes, C. Rizos, J. Wang, D. Small, G. Voigt, and N. Gambale. High precision indoor and outdoor positioning using LocataNet. Journal of Global Positioning System, 2(2):73-82, January 2003. ISSN 1446-3156. (Cited on page 25).

[17] S. Bellofiore, C. A. Balanis, J. Foutz, and A. S. Spanias. Smart-antenna systems for mobile communication networks - part 1: overview and antenna design. IEEE Antennas and Propagation Magazine, 44(3):145-154, May 2002. ISSN 1045-9243. doi: 10.1109/MAP.2002.1039395. (Cited on page 2).

[18] S. Bellofiore, C. A. Balanis, J. Foutz, and A. S. Spanias. Smart-antenna systems for mobile communication networks - part 2: beamforming and network throughput. IEEE Antennas and Propagation Magazine, 44(4):106-114, August 2002. ISSN 10459243. doi: 10.1109/MAP.2002.1043158. (Cited on pages 2 and 5).

[19] D. Bertsimas and J. Tsitsiklis. Simulated annealing. Statistical Science, 8(1):10-15, June 1993. ISSN 0764-6073. doi: 10.1214/ss/1177011077. (Cited on page 51).

[20] V. Q. Bien. Handoff management in radio over giber $60 \mathrm{GHz}$ indoor networks. $\mathrm{PhD}$ thesis, Technische Universiteit Delft, Delft, November 2014. (Cited on pages 62, 71, and 129).

[21] K. C. H. Blom. Blind equalization for underwater communications. $\mathrm{PhD}$ thesis, Universeit Twente, Enschede, July 2014. (Cited on pages 13 and 24).

[22] D. W. Boeringer and D. H. Werner. Particle swarm optimization versus genetic algorithms for phased array synthesis. IEEE Transactions on Antennas and Propagation, 52(3):771-779, March 2004. ISSN 0018-926X. doi: 10.1109/TAP.2004.825102. (Cited on page 51 ). 
[23] A. J. Booysen. Circular aperture pattern synthesis from collapsed equivalent linesource distributions. IEEE Transactions on Antennas and Propagation, 52(11):29042911, November 2004. ISSN 0018-926X. doi: 10.1109/TAP.2004.834380. (Cited on pages 101, 103, and 106).

[24] Y. Bruck and L. Sodin. On the ambiguity of the image reconstruction problem. Optics Communications, 30(3):304-308, September 1979. ISSN 00304018. doi: 10.1016/00304018(79)90358-4. (Cited on page 95).

[25] O. M. Bucci, G. D’Elia, G. Mazzarella, and G. Panariello. Antenna pattern synthesis: a new general approach. Proceedings of the IEEE, 82(3):358-371, March 1994. ISSN o018-9219. doi: 10.1109/5.272140. (Cited on pages 50 and 100).

[26] O. M. Bucci, G. D’Elia, and G. Romito. Power synthesis of conformal arrays by a generalised projection method. IEE Proceedings of Microwaves, Antennas and Propagation, 142(6):467-471, December 1995. ISSN 1350-2417. doi: 10.1049/ip-map:1995229o. (Cited on page 95).

[27] A. Cantoni. Application of orthogonal perturbation sequences to adaptive beamforming. IEEE Transactions on Antennas and Propagation, 28(2):191-202, March 1980. ISSN o018-926X. doi: 10.1109/TAP.1980.1142299. (Cited on page 24).

[28] Z. Cao. Radio beam steering in indoor fibre-wireless networks. $\mathrm{PhD}$ thesis, Technische Universiteit Eindhoven, Eindhoven, April 2015. (Cited on pages 4, 20, 26, and 129).

[29] Z. Cao, Q. Wang, R. Lu, A. C. F. Reniers, H. P. A. v. d. Boom, E. Tangdiongga, and A. M. J. Koonen. Microwave beam steering with tunable spectral filtering using cyclic additional optical true time delay. In Optical Fiber Communication, March 2014. (Cited on pages 20 and 77).

[30] J. Capmany, B. Ortega, and D. Pastor. A tutorial on microwave photonic filters. Journal of Lightwave Technology, 24(1):201-229, January 2006. ISSN 0733-8724. doi: 10.1109/JLT.2005.860478. (Cited on pages 19 and 20).

[31] J. Capon. High-resolution frequency-wavenumber spectrum analysis. Proceedings of the IEEE, 57(8):1408-1418, August 1969. ISSN 0018-9219. doi: 10.1109/PROC.1969.7278. (Cited on page 23).

[32] K. Chandra, R. V. Prasad, B. Quang, and I. G. M. M. Niemegeers. CogCell: Cognitive interplay between $60 \mathrm{GHz}$ picocells and $2.4 / 5 \mathrm{GHz}$ hotspots in the $5 \mathrm{G}$ era. IEEE Communications Magazine, Special issue on Emerging Applications, Services and Engineering for Cognitive Cellular Systems (EASE4CCS), July 2015. (Cited on page 129).

[33] S. Chandran. Adaptive antenna arrays: trends and applications, chapter 3. SpringerVerlag, 1 edition, June 2004. ISBN 3540201998. (Cited on page 55).

[34] J. L. Corral, J. Marti, S. Regidor, J. M. Foster, R. Laming, and M. J. Cole. Continuously variable true time-delay optical feeder for phased-array antenna employing chirped fiber grating. IEEE Transactions on Microwave Theory and Techniques, 45(8):1531-536, August 1997. ISSN 0018-9480. doi: 10.1109/22.618471. (Cited on page 19). 
[35] CST. Microwave Studio. https://www.cst.com/Products/CSTMWS. Accessed: 15-11-2015. (Cited on page 63).

[36] B. L. Dang, M. G. Larrode, R. V. Prasad, I. G. M. M. Niemegeers, and A. M. J. Koonen. Radio-over-fiber based architecture for seamless wireless indoor communication in the $60 \mathrm{GHz}$ band. Computer Communications, 30(18):3598-3613, December 2007. ISSN 0140-3664. doi: 10.1016/j.comcom.2007.08.041. (Cited on pages 3 and 4).

[37] S. Denno and T. Ohira. Modified constant modulus algorithm for digital signal processing adaptive antennas with microwave analog beamforming. IEEE Transactions on Antennas and Propagation, 50(6):850-857, June 2002. ISSN 0018-926X. doi: 10.1109/TAP.2002.1017667. (Cited on page 24).

[38] A. W. Doff, K. Chandra, and R. V. Prasad. Sensor assisted movement identification and prediction for beamformed $60 \mathrm{GHz}$ links. In 12th IEEE Consumer Communications and Networking Conference (CCNC), Las Vegas, USA, January 2015. (Cited on page 129).

[39] C. L. Dolph. A current distribution for broadside arrays which optimizes the relationship between beam width and side-lobe level. Proceedings of the IRE, 34(6):335-348, June 1946. ISSN oo96-8390. doi: 10.1109/JRPROC.1946.225956. (Cited on page 107).

[40] R. S. Elliott. Improved pattern synthesis for equispaced linear arrays. Alta Frequenza rivista di radiotecnica, telefonia e acustica applicata, 51(1):296-300, November 1982. ISSN 0002-6557. (Cited on pages 34, 35, and 108).

[41] R. S. Elliott. Array pattern synthesis part II: planar arrays. IEEE Antennas and Propagation Society Newsletter, 28(2):4-10, April 1986. ISSN 2168-0329. doi: 10.1109/MAP.1986.27849. (Cited on pages 74, 101, and 103).

[42] R. S. Elliott. Criticisms of the Woodward-Lawson method. IEEE Antennas and Propagation Society Newsletter, 30(3):43, June 1988. ISSN 2168-0329. doi: 10.1109/MAP.1988.6079081. (Cited on page 50).

[43] R. S. Elliott. Antenna Theory and Design, Revised Edition. Wiley-IEEE Press, 1 edition, January 2003. ISBN 0471449962. (Cited on pages 7, 9, 13, 26, 28, 95, 101, and 107).

[44] R. S. Elliott and G. J. Stern. A new technique for shaped beam synthesis of equispaced arrays. IEEE Transactions on Antennas and Propagation, 32(10):1129-1133, October 1984. ISSN oo18-926X. doi: 10.1109/TAP.1984.1143216. (Cited on pages 34, 37, 38, 39, and 84 ).

[45] S. Emami. UWB communication systems: conventional and 60 GHz: principles, design and standards. Springer New York, April 2013. ISBN 9781461467533. (Cited on page 63).

[46] D. T. Emerson. The work of Jagadis Chandra Bose: 100 years of millimeter-wave research. IEEE Transactions on Microwave Theory and Techniques, 45(12):2267-2273, December 1997. ISSN 0018-9480. doi: 10.1109/22.643830. (Cited on page 1).

[47] S. Farzaneh and A. Sebak. A novel amplitude-phase weighting for analog microwave beamforming. IEEE Transactions on Antennas and Propagation, 54(7):1997-2008, July 2006. ISSN o018-926X. doi: 10.1109/TAP.2006.877160. (Cited on page 24). 
[48] J. A. Ferreira and F. J. Ares-Pena. Pattern synthesis of conformal arrays by the simulated annealing technique. Electronics Letters, 33(14):1187-1189, July 1997. ISSN oo13-5194. doi: 10.1049/el:19970838. (Cited on page 51).

[49] J. A. Fessler and A. O. Hero. Space-alternating generalized expectation-maximization algorithm. EEE Transactions on Signal Processing, 42(10):2664-2677, October 1994. ISSN 1053-587X. doi: 10.1109/78.324732. (Cited on page 23).

[50] M. Y. Frankel and R. D. Esman. True time-delay fiber-optic control of an ultrawideband array transmitter/receiver with multibeam capability. IEEE Transactions on Microwave Theory and Techniques, 43(9):2387-2394, September 1995. ISSN oo189480. doi: 10.1109/22.414593. (Cited on page 20).

[51] B. Fuchs. Shaped beam synthesis of arbitrary arrays via linear programming. IEEE Antennas and Wireless Propagation Letters, 9:481-484, June 2010. ISSN 1536-1225. doi: 10.1109/LAWP.2010.2051210. (Cited on pages 51, 73, and 95).

[52] T. Fung-I and D. K. Cheng. Optimum scannable planar arrays with an invariant sidelobe level. Proceedings of the IEEE, 56(11):1771-1778, November 1968. ISSN 0018-9219. doi: 10.1109/PROC.1968.6749. (Cited on page 107).

[53] Z. Genc, U. H. Rizvi, E. Onur, and I. G. M. M. Niemegeers. Robust $60 \mathrm{GHz}$ indoor connectivity: is it possible with reflections? In IEEE Vehicular Technology Conference, pages 1-5, May 2010. doi: 10.1109/VETECS.2010.5493722. (Cited on pages 71 and 129).

[54] D. Gies and Y. Rahmat-Samii. Particle swarm optimization for reconfigurable phasedifferentiated array design. Microwave and Optical Technology Letters, 38(3):168-175, January 2003. ISSN 1098-2760. doi: 10.1002/mop.11005. (Cited on page 51).

[55] L. Godara. Application of antenna arrays to mobile communications - part II: beamforming and direction-of-arrival considerations. Proceedings of the IEEE, 85(8):11951245, August 1997. ISSN 0018-9219. doi: 10.1109/5.622504. (Cited on pages 18, 22, and 55).

[56] L. C. Godara. Smart antennas. CRC Press, 1 edition, 2004. ISBN 9780203496770. (Cited on page 24).

[57] N. Goto. Pattern synthesis of hexagonal planar arrays. IEEE Transactions on Antennas and Propagation, 20(4):479-481, July 1972. ISSN o018-926X. doi: 10.1109/TAP.1972.1140247. (Cited on page 101).

[58] G. Grosskopf, R. Eggemann, S. Zinal, B. Kuhlow, G. Przyrembel, D. Rohde, A. Kortke, and $\mathrm{H}$. Ehlers. Photonic $60 \mathrm{GHz}$ maximum directivity beam former for smart antennas in mobile broad-band communications. IEEE Photonics Technology Letters, 14(8):1169-1171, August 2002. ISSN 1041-1135. doi: 10.1109/LPT.2002.1022007. (Cited on page 19).

[59] R. C. Hansen. Microwave scanning antennas. Academic Press, 1966. ISBN 9780685231111. (Cited on page 21).

[6o] R. C. Hansen. Decreasing antenna beamwidth with increasing scan angle. Electronics Letters, 6(17):544-545, August 1970. ISSN 0013-5194. doi: 10.1049/el:19700378. (Cited on page 61). 
[61] P. Henry and H. Luo. WiFi: what's next? IEEE Communications Magazine, 40(12): 66-72, December 2002. ISSN 0163-6804. (Cited on page 1).

[62] F. Herrera, M. Lozano, and J. L. Verdegay. Tackling real-coded genetic algorithms: operators and tools for behavioural analysis. Artificial Intelligence Review, 12(4): 265-319, August 1998. ISSN 0269-2821. doi: 10.1023/A:1006504901164. (Cited on page 51).

[63] J. H. Holland. Genetic algorithms. Scientific american, 267(1):66-72, 1992. (Cited on page 51).

[64] C. Hsi-Tseng, W. Nan-Nan, C. Hsi-Hsir, and Q. Jing-Hui. An effective synthesis of planar array antennas for producing near-field contoured patterns. IEEE Transactions on Antennas and Propagation, 59(9):3224-3233, September 2011. ISSN oo18-926X. doi: 10.1109/TAP.2011.2161554. (Cited on pages 50 and 101).

[65] D. B. Hunter, M. E. Parker, and J. L. Dexter. Demonstration of a continuously variable true-time delay beamformer using a multichannel chirped fiber grating. IEEE Transactions on Microwave Theory and Techniques, 54(2):861-867, February 2006. ISSN 0018-9480. doi: 10.1109/TMTT.2005.863056. (Cited on page 20).

[66] R. F. Hyneman and R. Johnson. A technique for the synthesis of shaped-beam radiation patterns with approximately equal-percentage ripple. IEEE Transactions on Antennas and Propagation, 15(6):736-743, November 1967. ISSN 0018-926X. doi: 10.1109/TAP.1967.1139040. (Cited on page 50).

[67] IEEE. Standard for information technology - telecommunications and information exchange between systems - local and metropolitan area networks - specific requirements - part 15.3: wireless medium access control (mac) and physical layer (phy) specifications for high rate wireless personal area networks (wpans) amendment 2: millimeter-wave-based alternative physical layer extension. IEEE Std 802.15.3C2009 (Amendment to IEEE Std 802.15.3-2003), pages 1-187, December 2009. doi: 10.1109/IEEESTD.2009.5284444. (Cited on pages 1, 3, 25, 59, 64, 71, and 129).

[68] IEEE. International standard for information technology - telecommunications and information exchange between systems - local and metropolitan area networks - specific requirements - part 11: Wireless lan medium access control (mac) and physical layer (phy) specifications amendment 3: Enhancements for very high throughput in the $60 \mathrm{GHz}$ band (adoption of IEEE std 802.11AD-2012). ISO/IEC/IEEE 880211:2012/Amd.3:2014(E), pages 1-634, March 2014. doi: 10.1109/IEEESTD.2014.6774849. (Cited on pages 1, 3, 71, and 129).

[69] J. Illade-Quinteiro, J. A. Rodriguez-Gonzalez, and F. J. Ares-Pena. Shapedpattern synthesis by spreading out collapsed distributions. IEEE Antennas and Propagation Magazine, 52(6):110-114, December 2010. ISSN 1045-9243. doi: 10.1109/MAP.2010.5723234. (Cited on pages 101, 105, 107, and 111).

[7o] M. F. Jahromi. Optical and microwave beamforming for phased array antennas. $\mathrm{PhD}$ thesis, University of Waterloo, Ontario, 2008. (Cited on page 24).

[71] S. M. K and M. Alouini. Digital communication over fading channels, second edition. Wiley-IEEE Press, 2 edition, Januari 2005. ISBN 9780471649533. (Cited on page 62). 
[72] Y. U. Kim and R. S. Elliott. Shaped-pattern synthesis using pure real distributions. IEEE Transactions on Antennas and Propagation, 36(11):1645-1649, November 1988. ISSN 0018-926X. doi: 10.1109/8.9721. (Cited on page 106).

[73] Y. U. Kim and R. S. Elliott. Extensions of the Tseng-Cheng pattern synthesis technique. Journal of Electromagnetic Waves and Applications, 2(3-4):255-268, 1988. doi: 10.1163/156939388Xoo224. (Cited on page 107).

[74] Y. U. Kim and J. D. Nespor. Shaped beam synthesis and conditional thinning for planar phased array. In Antennas and Propagation Society International Symposium, 1996. AP-S. Digest, volume 2, pages 802-805, July 1996. doi: 10.1109/APS.1996.549717. (Cited on pages 110 and 118).

[75] S. Kirkpatrick, C. D. Gelatt, and M. P. Vecchi. Optimization by simulated annealing. Science, 220(4598):671-680, 1983. (Cited on page 51).

[76] A. M. J. Koonen and M. G. Larrode. Radio-over-MMF techniques - part II: microwave to millimeter-wave systems. Journal of Lightwave Technology, 26(15):23962408, August 2008. ISSN 0733-8724. doi: 10.1109/JLT.2008.927182. (Cited on page 3).

[77] H. Lebret and S. Boyd. Antenna array pattern synthesis via convex optimization. IEEE Transactions on Signal Processing, 45(3):526-532, March 1997. ISSN 1053-587X. doi: $10.1109 / 78.558465$. (Cited on page 50 ).

[78] G. Liang, W. Gong, H. Liu, and J. Yu. Development of 61-channel digital beamforming (DBF) transmitter array for mobile satellite communication. Progress In Electromagnetics Research, 97:177-195, October 2009. ISSN 0908-2303. doi: 10.2528/PIERo9o82303. (Cited on page 99).

[79] K. R. Mahmoud, M. El-Adawy, S. M. M. Ibrahem, R. Bansal, and S. Zainud-Deen. A comparison between circular and hexagonal array geometries for smart antenna systems using particle swarm optimization algorithm. Progress In Electromagnetics Research, 72:75-90, April 2007. ISSN 1070-4698. doi: 10.2528/PIERo7030904. (Cited on page 98 ).

[80] R. J. Mailloux. Phased Array Antenna Handbook. Artech, 2 edition, March 2005. ISBN 9781580536899. (Cited on page 20).

[81] D. Marcano and F. Duran. Synthesis of antenna arrays using genetic algorithms. IEEE Antennas and Propagation Magazine, 42(3):12-20, June 2000. ISSN 1045-9243. doi: 10.1109/74.848944. (Cited on pages 51 and 52).

[82] M. Marcus and B. Pattan. Millimeter wave propagation: spectrum management implications. IEEE Microwave Magazine, 6(2):54-62, June 2005. ISSN 1527-3342. doi: 10.1109/MMW.2005.1491267. (Cited on page 1).

[83] P. F. McManamon, T. A. Dorschner, D. L. Corkum, L. J. Friedman, D. S. Hobbs, M. Holz, S. Liberman, H. Q. Nguyen, D. P. Resler, R. C. Sharp, and E. A. Watson. Optical phased array technology. Proceedings of the IEEE, 84(2):268-298, February 1996. ISSN oo18-9219. doi: 10.1109/5.482231. (Cited on page 19). 
[84] A. Meijerink, C. Roeloffzen, R. Meijerink, L. Zhuang, D. Marpaung, M. Bentum, M. Burla, J. Verpoorte, P. Jorna, A. Hulzinga, and W. van Etten. Novel ring resonatorbased integrated photonic beamformer for broadband phased array receive antennas - part I: design and performance analysis. Journal of Lightwave Technology, 28(1):3-18, January 2010. ISSN 0733-8724. doi: 10.1109/JLT.2009.2029705. (Cited on page 19).

[85] R. A. Meyer. Optical beam steering using a multichannel lithium tantalate crystal. Applied optics, 11(3):613-616, March 1972. ISSN ooo3-6935. (Cited on page 19).

[86] T. A. Milligan. Modern antenna design. John Wiley \& Sons, July 2005. ISBN 9780471720607. (Cited on pages 31, 43, and 83).

[87] N. K. Nahar, B. Raines, R. G. Rojas, and B. Strojny. Wideband antenna array beam steering with free-space optical true-time delay engine. IET Microwaves, Antennas \& Propagation, 5(6):740-746, April 2011. ISSN 1751-8725. doi: 10.1049/ietmap.2010.0251. (Cited on page 20).

[88] W. Ng, A. A. Walston, G. L. Tangonan, J. J. Lee, I. L. Newberg, and N. Bernstein. The first demonstration of an optically steered microwave phased array antenna using true-time-delay. Lightwave Technology, Journal of, 9(9):1124-1131, September 1991. ISSN 0733-8724. doi: 10.1109/50.85809. (Cited on page 21).

[89] Y. Niu, Y. Li, D. Jin, L. Su, and D. Wu. Blockage robust and efficient scheduling for directional mmWave WPANs. IEEE Transactions on Vehicular Technology, 64(2): 728-742, February 2015. ISSN 0018-9545. doi: 10.1109/TVT.2014.232266o. (Cited on pages 71 and 129).

[9o] H. Oraizi and M. Fallahpour. Array pattern synthesis with mutual coupling consideration. In International Symposium on Telecommunications, pages 77-82, August 2008. doi: 10.1109/ISTEL.2008.4651275. (Cited on pages 73, 83, and 84).

[91] H. J. Orchard, R. S. Elliott, and G. J. Stern. Optimising the synthesis of shaped beam antenna patterns. IEEE Proceedings of Microwaves, Antennas and Propagation, 132 (1):63-68, February 1985. ISSN 0950-107X. doi: 10.1049/ip-h-2.1985.0o13. (Cited on pages $28,34,40,41,44,45,46,76,82,84,87,101,104$, and 116).

[92] A. Paulraj, R. Roy, and T. Kailath. Estimation of signal parameters via rotational invariance techniques - ESPRIT. In Asilomar Conference on Circuits, Systems and Computers, pages 83-89, July 1985. doi: 10.1109/ACSSC.1985.671426. (Cited on page 23).

[93] M. Pichler, S. Schwarzer, A. Stelzer, and M. Vossiek. Positioning with moving IEEE 802.15.4 (ZigBee) transponders. In IEEE MTT-S International Microwave Workshop on Wireless Sensing, Local Positioning, and RFID, pages 1-4, September 2009. doi: 10.1109/IMWS2.2009.5307878. (Cited on page 25).

[94] M. Piqueras, G. Grosskopf, B. Vidal, J. Herrera, J. Martinez, P. Sanchis, V. Polo, J. Corral, A. Marceaux, J. Galiere, J. Lopez, A. Enard, J.-L. Valard, O. Parillaud, E. Estebe, N. Vodjdani, M.-S. Choi, J. den Besten, F. Soares, M. Smit, and J. Marti. Optically beamformed beam-switched adaptive antennas for fixed and mobile broad-band wireless access networks. IEEE Transactions on Microwave Theory and Techniques, 54(2):887-899, February 2006. ISSN 0018-9480. doi: 10.1109/TMTT.2005.863049. (Cited on pages 19, 20, 21, 60, and 88). 
[95] S. Rajagopal, S. Abu-Surra, P. Zhouyue, and F. Khan. Antenna array design for multi-Gbps mmWave mobile broadband communication. In IEEE Global Telecommunications Conference (GLOBECOM), pages 1-6, December 2011. doi: 10.1109/GLOCOM.2011.6133699. (Cited on page 56).

[96] M. Rammal, D. Eclercy, A. Reineix, and B. Jecko. Study of mutual coupling effect on radiated patterns of antenna arrays. IEE Proceedings Microwaves, Antennas and Propagation, 144(5):389-391, October 1997. ISSN 1350-2417. doi: 10.1049/ip-map:19971231. (Cited on page 73).

[97] T. Rappaport, W. Roh, and C. Kyungwhoon. Mobile's millimeter-wave makeover. IEEE Spectrum, 51(9):34-58, September 2014. ISSN 0018-9235. doi: 10.1109/MSPEC.2014.6882985. (Cited on pages 1 and 2).

[98] O. Raz, S. Barzilay, R. Rotman, and M. Tur. Submicrosecond scan-angle switching photonic beamformer with flat RF response in the $\mathrm{C}$ and $\mathrm{X}$ bands. Journal of Lightwave Technology, 26(15):2774-2781, August 2008. ISSN 0733-8724. doi: 10.1109/JLT.2008.927150. (Cited on pages 20, 60, and 64).

[99] C. Reck, U. Berold, and L. P. Schmidt. Automated synthesis of shaped beam antenna patterns with implied cross coupling. In European Conference on Antennas and Propagation (EuCAP), pages 1-4, April 2010. (Cited on pages 73, 83, and 84).

[10o] R. Rehman and I. C. F. Ipsen. Computing characteristic polynomials from eigenvalues. SIAM Journal on Matrix Analysis and Applications, 32(1):90-114, 2011. ISSN 0895-4798. doi: 10.1137/100788392. (Cited on page 117).

[101] N. Rendevski and D. Cassioli. BER of IEEE 802.11AD OFDM radios vs. carrier frequency in real $60 \mathrm{GHz}$ indoor channels. In IEEE International Conference on Communications (ICC), pages 5878-5883, June 2014. doi: 10.1109/ICC.2014.6884260. (Cited on page 63).

[102] J. A. Rodriguez, R. Munoz, H. Estevez, F. J. Ares-Pena, and E. Moreno. Synthesis of planar arrays with arbitrary geometry generating arbitrary footprint patterns. IEEE Transactions on Antennas and Propagation, 52(9):2484-2488, September 2004. ISSN o018-926X. doi: 10.1109/TAP.2004.834125. (Cited on page 101).

[103] Rohde \& Schwarz. NRP-Z57 thermal power sensor. http://www. rohde-schwarz . nl/file/NRP-Family_dat-sw_en_5213-5539-22_v1000.pdf. Accessed: 15-112015. (Cited on pages 46 and 64).

[104] Y. S and C. J. Scheytt. Low-power $60 \mathrm{GHz}$ receiver front-end with a variable-gain LNA in SiGe BiCMOS technology. In IEEE Bipolar/BiCMOS Circuits and Technology Meeting, pages 192-195, October 2010. doi: 10.1109/BIPOL.2010.5668052. (Cited on page 85).

[105] M. G. Sanchez, A. V. Alejos, and I. Cuinas. Indoor radio reception at 60 GHz: fading mitigation using diversity techniques. IEEE Transactions on Antennas and Propagation, 57(10):2934-2939, October 2009. ISSN 0018-926X. doi: 10.1109/TAP.2009.2029279. (Cited on page 56). 
[106] P. Sanchis, V. Polo, J. Herrera, J. L. Corral, M.-S. C, and J. Marti. Experimental demonstration of a direction of arrival estimation algorithm for mm-wave broadband communication systems. In IEEE MTT-S International Microwave Symposium Digest, pages 1533-1536, June 2003. doi: 10.1109/MWSYM.2003.1210428. (Cited on page 19).

[107] S. A. Schelkunoff. A mathematical theory of linear arrays. Bell System Technical Journal, 22(1):80-107, Januari 1943. (Cited on pages 30 and 31).

[108] H. Schippers, J. Verpoorte, P. Jorna, A. Hulzinga, A. Meijerink, C. G. H. Roeloffzen, L. Zhuang, D. A. I. Marpaung, W. v. Etten, R. G. Heideman, A. Leinse, A. Borreman, M. Hoekman, and M. Wintels. Broadband conformal phased array with optical beam forming for airborne satellite communication. In Proceedings of IEEE Aerospace Conference 2008, pages 1-17, May 2008. (Cited on pages 19 and 20).

[109] R. O. Schmidt. Multiple emitter location and signal parameter estimation. IEEE Transactions on Antennas and Propagation, 34(3):276-280, March 1986. ISSN oo18926X. doi: 10.1109/TAP.1986.1143830. (Cited on page 23).

[110] SiBeam. OmniLink 60. http://www. sibeam. com. Accessed: 15-11-2015. (Cited on page 3).

[111] S. M. Shinners. Modern control system theory and design. John Wiley \& Sons, September 1998. ISBN 9780471249061. (Cited on pages 31 and 61).

[112] M. T. M. Silva and V. H. Nascimento. Tracking analysis of the constant modulus algorithm. In IEEE International Conference on Acoustics, Speech and Signal Processing, pages 3561-3564, March 2008. doi: 10.1109/ICASSP.2008.4518421. (Cited on page 23).

[113] M. Skolnik. Radar handbook. McGraw-Hill, 3 edition, 2008. ISBN 0071485473. (Cited on page 17).

[114] P. Smulders. Exploiting the $60 \mathrm{GHz}$ band for local wireless multimedia access: prospects and future directions. IEEE Communications Magazine, 40(1):140-147, January 2002. ISSN 0163-6804. doi: 10.1109/35.978061. (Cited on page 63).

[115] H. Song, G. Liang, W. Gong, and J. Yu. A comparison between circular and hexagonal array geometries for smart antenna systems using particle swarm optimization algorithm. Progress In Electromagnetics Research, 145:141-151, March 2014. ISSN 1401-0604. doi: 10.2528/PIER14010604. (Cited on page 99).

[116] R. A. Soref. Optical dispersion technique for time-delay beam steering. Applied Optics, 31(35):7395-7397, December 1992. doi: 10.1364/AO.31.007395. (Cited on page 20).

[117] W. L. Stutzman and G. A. Thiele. Antenna theory and design. Wiley-Interscience, 1 edition, 1981. ISBN 047104458. (Cited on page 11).

[118] C. Sun and N. C. Karmakar. Direction of arrival estimation with a novel single-port smart antenna. EURASIP Journal on Advances in Signal Processing, 2004:1364-1375, January 2004. ISSN 1110-8657. doi: 10.1155/S111086570431108X. (Cited on page 24). 
[119] Y. M. Tao and G. Y. Delisle. Lens-fed multiple beam array for millimeter wave indoor communications. In IEEE Antennas and Propagation Society International Symposium, volume 4, pages 2206-2209, July 1997. doi: 10.1109/APS.1997.625407. (Cited on page 21).

[120] T. T. Taylor. Design of line-source antennas for narrow beamwidth and low side lobes. Transactions of the IRE Professional Group on Antennas and Propagation, 3(1): 16-28, Januari 1955. ISSN 2168-0639. doi: 10.1109/TPGAP.1955.5720407. (Cited on page 87 ).

[121] Texas Instruments. ADC12D16ooRF. http://www.ti.com/product/ adc12d1600rf. Accessed: 15-11-2015. (Cited on page 55).

[122] A. J. van der Veen. Algebraic methods for deterministic blind beamforming. Proceedings of the IEEE, 86(10):1987-2008, October 1998. ISSN 0018-9219. doi: 10.1109/5.720249. (Cited on page 24).

[123] H. L. van Trees. Optimum array processing (detection, estimation, and modulation theory, part IV). Wiley-Interscience, 1 edition, March 2002. ISBN 0471093904. (Cited on pages $7,9,10,13,16,17,26,28,30,47,74,75,78,94,95$, and 98).

[124] B. D. Van Veen and K. M. Buckley. Beamforming: a versatile approach to spatial filtering. IEEE ASSP Magazine, 5(2):4-24, April 1988. ISSN 0740-7467. doi: 10.1109/53.665. (Cited on page 18).

[125] A. Varshavsky, E. de Lara, J. Hightower, A. LaMarca, and V. Otsason. GSM indoor localization. Pervasive and Mobile Computing, 3(6):698-720, December 2007. ISSN 1574-1192. doi: 10.1016/j.pmcj.2007.07.004. (Cited on page 25).

[126] L. I. Vaskelainen. Constrained least-squares optimization in conformal array antenna synthesis. IEEE Transactions on Antennas and Propagation, 55(3):859-867, March 2007. ISSN 0018-926X. doi: 10.1109/TAP.2007.891860. (Cited on pages 50, 73, and 101).

[127] F. J. Villegas. Parallel genetic-algorithm optimization of shaped beam coverage areas using planar 2-d phased arrays. IEEE Transactions on Antennas and Propagation, 55 (6):1745-1753, June 2007. ISSN oo18-926X. doi: 10.1109/TAP.2007.898601. (Cited on pages 51, 52, and 100).

[128] J. Wang, Z. Lan, C. Pyo, T. Baykas, C. Sum, M. A. Rahman, J. Gao, R. Funada, F. Kojima, H. Harada, and S. Kato. Beam codebook based beamforming protocol for multi-Gbps millimeter-wave WPAN systems. IEEE Journal on Selected Areas in Communications, 27(8):1390-1399, October 2009. ISSN 0733-8716. doi: 10.1109/JSAC.2009.091009. (Cited on pages 6 and 56).

[129] J. Wang, H. Zhang, T. Lv, and T. Aaron Gulliver. Capacity of $60 \mathrm{GHz}$ wireless communication systems over fading channels. Journal of Networks, 7(1), January 2012. ISSN 1796-2056. doi: 10.4304/jnw.7.1.203-209. (Cited on page 62).

[130] B. Widrow and J. M. McCool. A comparison of adaptive algorithms based on the methods of steepest descent and random search. IEEE Transactions on Antennas and Propagation, 24(5):615-637, September 1976. ISSN 0018-926X. doi: 10.1109/TAP.1976.1141414. (Cited on page 24). 
[131] J. H. Wilkinson. Rounding errors in algebraic processes. Englewood Cliffs, New Jersey: Prentice Hall, 1 edition, 1963. ISBN 0486679993. (Cited on page 118).

[132] P. M. Woodward. A method of calculating the field over a plane aperture required to produce a given polar diagram. Journal of the Institution of Electrical Engineers - part IIIA: Radiolocation, 93(10):1554-1558, September 1946. doi: 10.1049/ji-3a-1.1946.0262. (Cited on page 50).

[133] P. M. Woodward and J. D. Lawson. The theoretical precision with which an arbitrary radiation-pattern may be obtained from a source of finite size. Journal of the Institution of Electrical Engineers - part III: Radio and Communication Engineering, 95(37): 363-370, September 1948. doi: 10.1049/ji-3-2.1948.0094. (Cited on page 50).

[134] N. R. Yousef and A. H. Sayed. A feedback analysis of the tracking performance of blind adaptive equalization algorithms. In Proceedings of the 38th IEEE Conference on Decision and Control, volume 1, pages 174-179, December 1999. doi: 10.1109/CDC.1999.832770. (Cited on page 23).

[135] L. Zhuang, C. G. H. Roeloffzen, R. G. Heideman, A. Borreman, A. Meijerink, and W. van Etten. Ring resonator-based single-chip $1 x 8$ optical beam forming network in LPCVD waveguide technology. In Proceedings of the IEEE/LEOS Benelux, pages 45-48, November 2006. (Cited on page 20).

[136] H. Zmuda, R. A. Soref, P. Payson, S. Johns, and E. N. Toughlian. Photonic beamformer for phased array antennas using a fiber grating prism. IEEE Photonics Technology Letters, 9(2):241-243, February 1997. ISSN 1041-1135. doi: 10.1109/68.553105. (Cited on page 20). 


\section{List of Publications}

[TB:1] T. M. Bruintjes, K. H. G. Walters, S. H. Gerez, E. Molenkamp, and G. J. M. Smit. Sabrewing: a lightweight architecture for combined floating-point and integer arithmetic. ACM Transactions on Architecture and Code Optimization, 8(4):41:1-41:22, January 2012. ISSN 1544-3566. doi: 10.1145/2086696.2086720.

[TB:2] T.M. Bruintjes, A.B.J. Kokkeler, G. Karagiannis, and Smit G.J.M. Survey of energy efficient tracking and localization techniques in buildings using optical and wireless communication media. In Multiple Access Communications, volume 7642 of Lecture Notes in Computer Science, pages 60-74, Maynooth, Ireland, November 2012. Springer Verlag. doi: 10.1007/978-3-642-34976-8_6.

[TB:3] S. Pande, M Fearghal, G.J.M. Smit, T.M. Bruintjes, J.H. Rutgers, S. Cawley, J. Harkin, and L. McDaid. Fixed latency on-chip interconnect for hardware spiking neural network architectures. Parallel Computing, 39(9):357-371, September 2013. ISSN 0167-8191. doi: 10.1016/j.parco.2013.04.010.

[TB:4] S. Pande, F. Morgan, S. Cawley, T.M. Bruintjes, G.J.M. Smit, McGinley B., S. Carrillo, J. Harkin, and L. McDaid. Modular neural tile architecture for compact embedded hardware spiking neural network. Neural Processing Letters, 37(2):131-153, October 2013. ISSN 1370-4621. doi: 10.1007/s11063-012-9274-5.

[TB:5] T.M. Bruintjes, A.B.J. Kokkeler, G. Karagiannis, and G.J.M. Smit. Using shaped beam patterns for tracking. IEEE Transactions on Antennas and Propagation, 62 (12):6496-6501, December 2014. ISSN 0018-926X. doi: 10.1109/TAP.2014.2360221.

[TB:6] T.M. Bruintjes, A.B.J. Kokkeler, G. Karagiannis, and G.J.M. Smit. Shaped pattern synthesis for equispaced linear arrays with non-isotropic antennas. In 9th European Conference on Antennas and Propagation, Lissabon, Portugal, April 2015.

[TB:7] K. Chandra, Z. Cao, T.M. Bruintjes, R.V. Prasad, G. Karagiannis, E. Tangdiongga, H.P.A. van den Boom, and A.B.J. Kokkeler. mCRAN: A radio access network architecture for $5 \mathrm{~g}$ indoor communications. In IEEE International Conference on Communications (ICC), pages 306-311, London, United Kingdom, June 2015. IEEE Communications Society. 
